\title{
Fe-catalyzed selective cyclopropanation of enynes under photochemical or thermal conditions
}

\author{
MSc. Marie-Idrissa Picher and Prof. Dr. Bernd Plietker* \\ Institute of Organic Chemistry, Department of Chemistry, University of Stuttgart, DE-70569 Stuttgart \\ bernd.plietker@oc.uni-stuttgart.de
}

\section{Table of Contents}

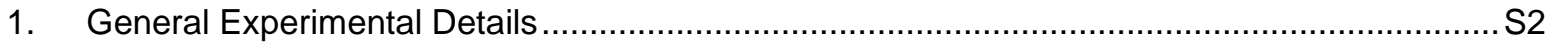

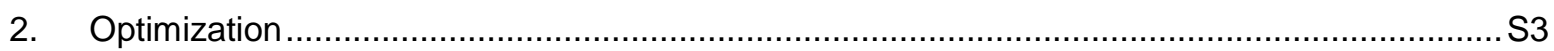

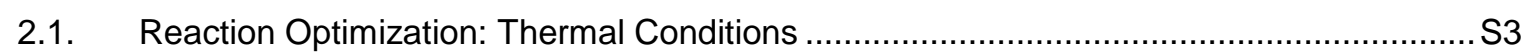

2.2. Reaction Optimization: Photochemical Conditions..................................................... 5

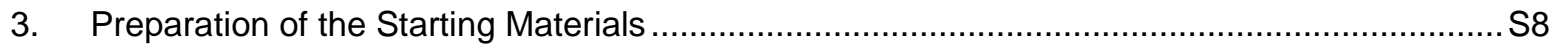

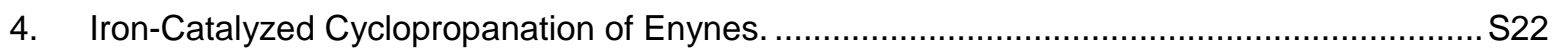

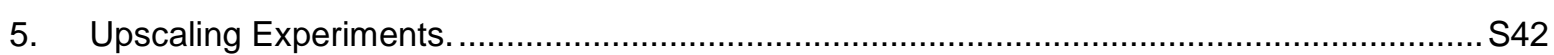

6. Iron-Catalyzed Cyclopropanation of Enyne 1 in the presence of TEMPO ….........................S43

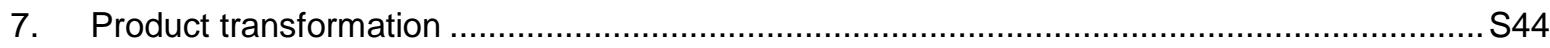

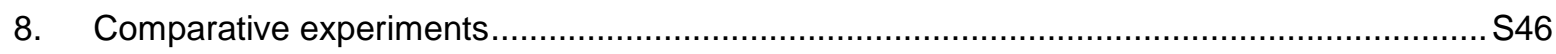

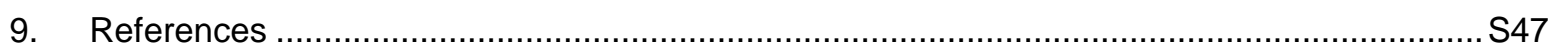

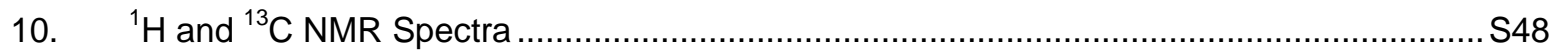




\section{General Experimental Details}

All reactions sensitive towards air and/or moisture were performed following standard Schlenk techniques using anhydrous solvents under dry nitrogen. Solvents were purified and dried prior to use. All chemicals were purchased from Acros Organics, Alfa Aesar, ChemPUR, Sigma Aldrich or TCl and used as received. TBA[Fe] $\left(\mathrm{Bu}_{4} \mathrm{~N}\left[\mathrm{Fe}(\mathrm{CO})_{3}(\mathrm{NO})\right]\right)$ was prepared according to a previously published literature procedure ${ }^{[1]}$. Ethyl diazoacetate 2 (containing $\leq 13 \mathrm{wt} \%$ dichloromethane) was purchased from Sigma-Aldrich and used as such. The photochemical reactions were carried out using a Heraeus Hg-Lamp ( $180 \mathrm{~W}$, cooled with a $5^{\circ} \mathrm{C}$ ethanol solution, at a distance of $3 \mathrm{~cm}$ ), a ORIEL Xenon-Lamp (75 W, at a distance of $47 \mathrm{~cm}$ ), or blue LEDs (405 nm). NMR spectra were recorded on a Bruker Avance 300 spectrometer at $300 \mathrm{MHz}\left({ }^{1} \mathrm{H}\right.$ NMR), and $75 \mathrm{MHz}\left({ }^{13} \mathrm{C} \mathrm{NMR}\right)$, a Bruker Ascend 400 spectrometer at $400 \mathrm{MHz}\left({ }^{1} \mathrm{H}\right.$ NMR), and $100 \mathrm{MHz}\left({ }^{13} \mathrm{C}\right.$ NMR) or a Bruker Avance 500 spectrometer at $500 \mathrm{MHz}\left({ }^{1} \mathrm{H}\right.$ NMR) and $125 \mathrm{MHz}\left({ }^{13} \mathrm{C}\right.$ NMR). Chemical shifts are reported in ppm and were corrected with the residual non-deuterated NMR solvent peaks. Coupling constants $J$ are given in $\mathrm{Hz}$. The following abbreviations are used in the analysis of NMR spectra: $s=s i n g l e t, d=d o u b l e t, t=t r i p l e t$, $\mathrm{q}=$ quartet, $\mathrm{m}=$ multiplet, $\mathrm{br}=$ broad. IR spectra were recorded on a FT-IR spectrometer in ATR mode. Band intensities are abbreviated as follows: weak $(\mathrm{w})$, medium $(\mathrm{m})$ and strong $(\mathrm{s})$. Mass spectra were measured using electrospray ionization or atmospheric pressure chemical ionization on a Bruker micrOTOF-Q (ESI) or electron impact on a Finnigan MAT-95 spectrometer (EI). High performance liquid chromatography (HPLC) was performed using a Knauer K-501 pump, a Knauer RI-detector K 2400 and a Macherey-Nagel VP250/21 Nucleodur 100-5 column, with a flow rate set to $10 \mathrm{~mL} / \mathrm{min}$. Xray structures were measured on a Bruker Kappa APEXII Duo diffractometer by Dr. Wolfgang Frey. Melting points were recorded on a Stuart SMP10 melting point apparatus and are uncorrected. 


\section{Optimization}

\subsection{Reaction Optimization: Thermal Conditions}

Table S1: Solvent screening.

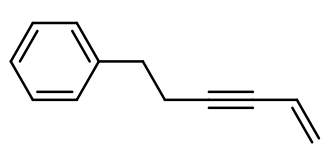

1
TBAFe [2.5 mol\%]

Ethyl diazoacetate 2 (1.05 eq.)

Solvent [2 M]

$40^{\circ} \mathrm{C}, 24 \mathrm{~h}$

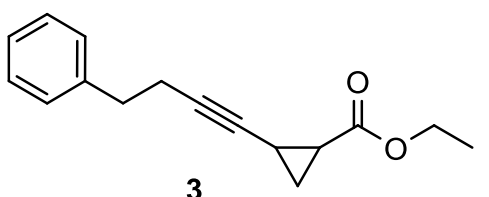

\begin{tabular}{|c|c|c|c|}
\hline Entry $^{[\mathrm{a}]}$ & Solvent mixture & Ratio & Yield $^{[b]}$ \\
\hline 1 & 1,2-DCE & - & $66 \%{ }^{[c]}$ \\
\hline 2 & 1,2-DCE:Nitromethane & $8: 2$ & $94 \%\left(81 \%{ }^{[\mathrm{c}]}\right)$ \\
\hline 3 & MeCN:Nitromethane & $8: 2$ & $69 \%$ \\
\hline 4 & Dioxane:Nitromethane & $8: 2$ & $74 \%$ \\
\hline 5 & THF:Nitromethane & $8: 2$ & $71 \%$ \\
\hline 6 & DCM:Nitromethane & $8: 2$ & $63 \%$ \\
\hline 7 & Toluene:Nitromethane & $8: 2$ & $68 \%$ \\
\hline 8 & MTBE:Nitromethane & $8: 2$ & $69 \%$ \\
\hline 9 & DMF:Nitromethane & $8: 2$ & $11 \%$ \\
\hline 10 & Dioxane:Nitromethane & $8: 2$ & $74 \%$ \\
\hline 11 & MeOH:Nitromethane & $8: 2$ & $35 \%$ \\
\hline 12 & 1,2-DCE:Nitromethane & $9: 1$ & $91 \%\left(82 \%{ }^{[c]}\right)$ \\
\hline 13 & 1,2-DCE:Nitromethane & $7: 3$ & $84 \%\left(79 \%{ }^{[\mathrm{c}]}\right)$ \\
\hline 14 & 1,2-DCE:Nitromethane & $1: 1$ & $81 \%\left(74 \%{ }^{[c]}\right)$ \\
\hline $15^{[\mathrm{d}]}$ & 1,2-DCE:Nitromethane & $9: 1$ & $40 \%$ \\
\hline $16^{[e]}$ & 1,2-DCE:Nitromethane & $9: 1$ & $55 \%$ \\
\hline $17^{[\mathrm{f}]}$ & 1,2-DCE:Nitromethane & $9: 1$ & $69 \%$ \\
\hline
\end{tabular}


Table S2: Additive screening.

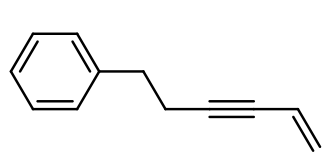

1

TBAFe [2.5 mol\%]

Ethyl diazoacetate 2 (1.05 eq.)

Additive

1,2-DCE [2 M]

$40^{\circ} \mathrm{C}, 24 \mathrm{~h}$

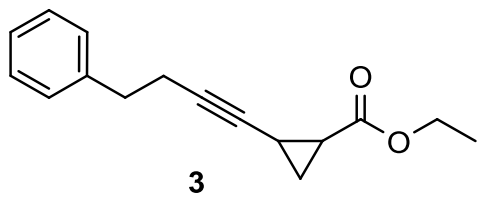

\begin{tabular}{llll} 
Entry $^{[a]}$ & Additive & Equivalents & Yield $^{[\mathrm{b}]}$ \\
\hline $\mathbf{1}$ & Nitromethane & 0.89 & $87 \%$ \\
$\mathbf{2}$ & Nitromethane & 0.5 & $80 \%$ \\
$\mathbf{3}$ & Nitromethane & 0.025 & $81 \%\left(79 \%{ }^{[\mathrm{c}]}\right)$ \\
$\mathbf{4}$ & Nitrobenzene & 0.89 & $85 \%$ \\
$\mathbf{5}$ & Nitrobenzene & 0.5 & $87 \%\left(81 \%{ }^{[\mathrm{c}]}\right)$ \\
$\mathbf{6}$ & 4-Nitrotoluene & 0.89 & $85 \%$ \\
$\mathbf{7}$ & 4-Nitrotoluene & 0.5 & $77 \%$ \\
$\mathbf{8}$ & 4-Nitroanisole & 0.89 & $77 \%$ \\
$\mathbf{9}$ & 4-Nitroanisole & 0.5 & $88 \%\left(76 \%{ }^{[\mathrm{c}]}\right)$ \\
$\mathbf{1 0}$ & 4-Nitroanisole & 0.025 & $81 \%\left(80 \%{ }^{[\mathrm{c}]}\right)$ \\
$\mathbf{1 1}$ & 4-Nitroanisole & 0.0125 & $80 \%\left(73 \%{ }^{[\mathrm{c}]}\right)$ \\
$\mathbf{1 2}$ & 4-Nitroanisole & $\mathbf{0 . 0 0 5}$ & $\mathbf{8 2} \%$ \\
$\mathbf{1 3}$ & TMANO & 0.005 & $61 \%$
\end{tabular}

${ }^{\text {[a] }}$ All reactions were performed on $0.25 \mathrm{mmol}$ scale in dry solvents at $40{ }^{\circ} \mathrm{C}$ under a nitrogen atmosphere for $24 \mathrm{~h}$; ${ }^{[b]}$ Yield determined by ${ }^{1} \mathrm{H}$ NMR integration using mesitylene as internal standard; ${ }^{[c]}$ Isolated yield.

Table S3: Blank reactions.

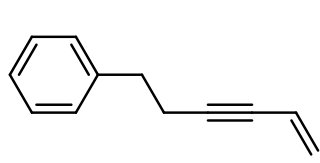

1
TBAFe [2.5 mol\%]

4-Nitroanisole [0.5 mol\%] Ethyl diazoacetate (1.05 eq.)

1,2-DCE [2 M]

$40^{\circ} \mathrm{C}, 24 \mathrm{~h}$

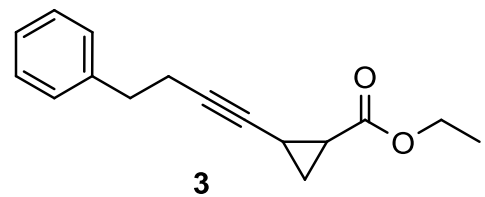

\begin{tabular}{lll} 
Entry $^{[\mathrm{a}]}$ & Deviations from original conditions & Yield $^{[\mathrm{b}]}$ \\
\hline $\mathbf{1}$ & None & $82 \%$ \\
$\mathbf{2}$ & No 4-nitroanisole & $68 \%$ \\
$\mathbf{3}$ & No TBA[Fe] & $15 \%$ \\
$\mathbf{4}$ & No 4-nitroanisole and no TBA[Fe] & $13 \%$ \\
\hline
\end{tabular}

${ }^{[a]}$ All reactions were performed on $0.25 \mathrm{mmol}$ scale in dry solvents at $40{ }^{\circ} \mathrm{C}$ under a nitrogen atmosphere for $24 \mathrm{~h}$; ${ }^{[b]}$ Yield determined by ${ }^{1} \mathrm{H}$ NMR integration using mesitylene as internal standard. 


\subsection{Reaction Optimization: Photochemical Conditions}

Table S4: Solvent screening.

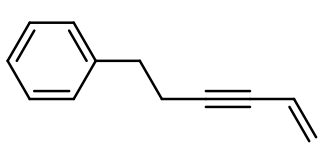

1

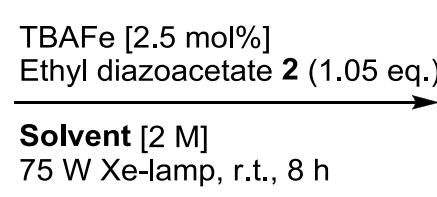

75 W Xe-lamp, r.t., $8 \mathrm{~h}$

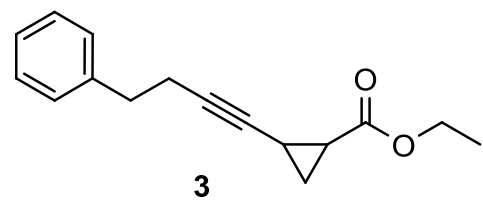

3

\begin{tabular}{llll} 
Entry $^{[\mathrm{ac}]}$ & Solvent mixture & Ratio & Yield $^{[\mathrm{b}]}$ \\
\hline $\mathbf{1}$ & 1,2-DCE & - & $34 \%{ }^{[\mathrm{c}]}$ \\
$\mathbf{2}$ & THF:Nitromethane & $8: 2$ & $34 \%$ \\
$\mathbf{3}$ & 1,2-DCE:Nitromethane & $8: 2$ & $35 \%$ \\
$\mathbf{4}$ & MeCN:Nitromethane & $8: 2$ & $49 \%\left(43 \%{ }^{[\mathrm{c}]}\right)$ \\
$\mathbf{5}$ & DCM:Nitromethane & $8: 2$ & $39 \%$ \\
$\mathbf{6}$ & Toluene:Nitromethane & $8: 2$ & $24 \%$ \\
$\mathbf{7}$ & MTBE:Nitromethane & $8: 2$ & $35 \%$ \\
$\mathbf{8}$ & DMF:Nitromethane & $8: 2$ & $38 \%$ \\
$\mathbf{9}$ & 1,4-Dioxane:Nitromethane & $8: 2$ & $51 \%\left(44 \%{ }^{[\mathrm{c}]}\right)$ \\
$\mathbf{1 0}$ & MeOH:Nitromethane & $8: 2$ & $22 \%$ \\
$\mathbf{1 1}$ & 1,4-Dioxane:Nitromethane & $9: 1$ & $46 \%$ \\
$\mathbf{1 2}$ & 1,4-Dioxane:Nitromethane & $7: 3$ & $39 \%$ \\
$\mathbf{1 3}$ & 1,4-Dioxane:Nitromethane & $8: 2$ & $20 \%$ \\
$\mathbf{1 4}$ & 1,4-Dioxane:Nitromethane & $8: 2$ & $66 \%\left(64 \%{ }^{[\mathrm{cc}]}\right)$ \\
$\mathbf{1 5}$ & 1,4-Dioxane:Nitromethane & $\mathbf{8 : 2}$ & $\mathbf{6 9} \%\left(\mathbf{6 2} \%{ }^{[\mathrm{cc}]}\right)$ \\
\hline${ }^{[a]}$ All & reactions & were performed on 0.25 mmol scale in dry solvents at room temperature under a
\end{tabular}
nitrogen atmosphere for $8 \mathrm{~h}$; ${ }^{[b]}$ Yield determined by ${ }^{1} \mathrm{H}$ NMR integration using mesitylene as internal standard; ${ }^{[\mathrm{c}]}$ Isolated yield; ${ }^{[\mathrm{d}]}$ Solvent concentration: [1 M]; ${ }^{[\mathrm{ej}}$ With $5 \mathrm{~mol} \% \mathrm{TBA}[\mathrm{Fe}] ;{ }^{[\mathrm{fl}} 16 \mathrm{~h}$.

Table S5: Additive screening.

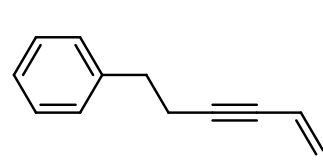

1
TBAFe [5 mol\%]

Ethyl diazoacetate 2 (1.05 eq.) Additive

1,4-Dioxane [2 M]

$75 \mathrm{~W}$ Xe-lamp, r.t., $16 \mathrm{~h}$

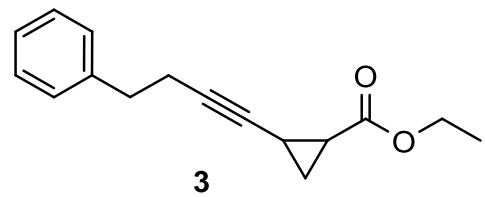

\begin{tabular}{llll} 
Entry $^{[\mathrm{a}]}$ & Additive & Equivalents & Yield $^{[\mathrm{b}]}$ \\
\hline $\mathbf{1}$ & Nitromethane & $\mathbf{1 . 8 5}$ & $\mathbf{7 9} \%\left(\mathbf{8 0} \%{ }^{[\mathrm{cc})}\right)$ \\
$\mathbf{2}$ & Nitromethane & 0.5 & $64 \%$ \\
$\mathbf{3}$ & 4-Nitroanisole & 0.5 & $33 \%$ \\
$\mathbf{4}$ & 4-Nitrotoluene & 0.5 & $23 \%$ \\
$\mathbf{5}$ & Nitrobenzene & 0.5 & $33 \%$ \\
\hline
\end{tabular}

${ }^{[a]}$ All reactions were performed on $0.25 \mathrm{mmol}$ scale in dry solvents at room temperature under a nitrogen atmosphere for $16 \mathrm{~h}$; ${ }^{[b]}$ Yield determined by ${ }^{1} \mathrm{H}$ NMR integration using mesitylene as internal standard; ${ }^{[c]}$ Isolated yield. 
Table S6: Blank reactions.

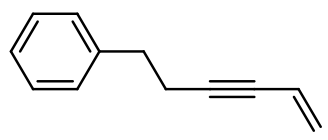

1

TBAFe [5 mol\%]

Ethyl diazoacetate 2 (1.05 eq.)

1,4-Dioxane:Nitromethane (5:1) [1.66 M]

$75 \mathrm{~W}$ Xe-lamp, r.t., $16 \mathrm{~h}$

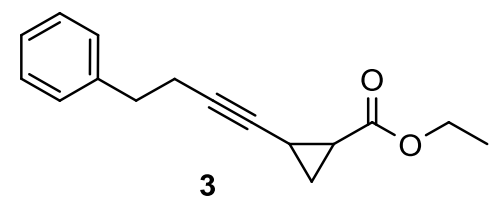

\begin{tabular}{lll} 
Entry $^{[\mathrm{a}]}$ & Deviations from original conditions & Yield $^{[\mathrm{b}]}$ \\
\hline $\mathbf{1}$ & none & $79 \%\left(80 \%{ }^{[\mathrm{c}]}\right)$ \\
$\mathbf{2}$ & No nitromethane & $73 \%$ \\
$\mathbf{3}$ & No TBA[Fe] & traces \\
$\mathbf{4}$ & No nitromethane and no TBA[Fe] & traces \\
$\mathbf{5}$ & Shielded from light & $43 \%$
\end{tabular}

${ }^{[a]}$ All reactions were performed on $0.25 \mathrm{mmol}$ scale in dry solvents at room temperature under a nitrogen atmosphere for $24 \mathrm{~h} ;{ }^{[b]}$ Yield determined by ${ }^{1} \mathrm{H}$ NMR integration using mesitylene as internal standard; ${ }^{\left[{ }^{[}\right]}$Isolated yield.

Upon upscaling from $0.25 \mathrm{mmol}$ to $0.50 \mathrm{mmol}$ scale under otherwise identical reaction conditions, the yields could not be reproduced. Thus, a brief optimization was carried out on $0.50 \mathrm{mmol}$ scale to achieve reproducible yields.

Table S7: Optimization on $0.50 \mathrm{mmol}$ scale.

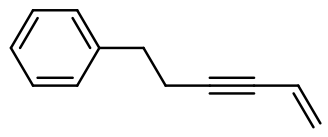

1
TBAFe [5 mol\%]

Ethyl diazoacetate 2 ( 1.05 eq.)

$\overrightarrow{\text { 1,4-Dioxane:Nitromethane (ratio) [X M] }}$ $75 \mathrm{~W}$ Xe-lamp, r.t., time

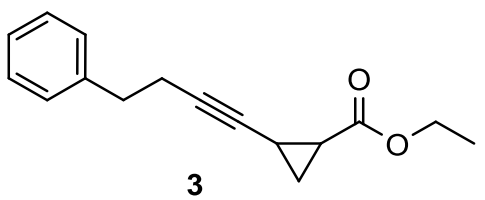

\begin{tabular}{lllll} 
Entry $^{[\mathrm{a}]}$ & Solvent ratio & Solvent concentration & Reaction time & Yield $^{[\mathrm{b}]}$ \\
\hline $\mathbf{1}$ & $5: 1$ & $1.66 \mathrm{M}$ & $16 \mathrm{~h}$ & $65 \%{ }^{[\mathrm{cc}]}$ \\
$\mathbf{2}$ & $10: 1$ & $1.82 \mathrm{M}$ & $16 \mathrm{~h}$ & $66 \%$ \\
$\mathbf{3}$ & $5: 1$ & $3.33 \mathrm{M}$ & $16 \mathrm{~h}$ & $70 \%$ \\
$\mathbf{4}$ & $2.5: 1$ & $2.86 \mathrm{M}$ & $16 \mathrm{~h}$ & $64 \%$ \\
$\mathbf{5}$ & $\mathbf{5 : 1}$ & $\mathbf{3 . 3 3} \mathbf{~ M}$ & $\mathbf{2 0 ~ h}$ & $\left.\mathbf{7 9 \% ( 8 1 \%} \%^{[\mathrm{c}]}\right)$ \\
\hline
\end{tabular}

${ }^{[a]}$ All reactions were performed on $0.50 \mathrm{mmol}$ scale in dry solvents at room temperature under a nitrogen atmosphere; ${ }^{[b]}$ Yield determined by ${ }^{1} \mathrm{H}$ NMR integration using mesitylene as internal standard; ${ }^{[c]}$ Isolated yield. 
Table S8: Light source screening.

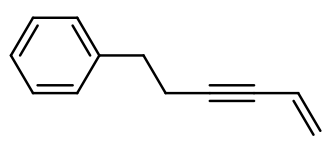

1
TBAFe [5 mol\%]

Ethyl diazoacetate 2 (1.05 eq.)

1,4-Dioxane:Nitromethane $(5: 1))$ [3.33 M] light source, r.t., $20 \mathrm{~h}$

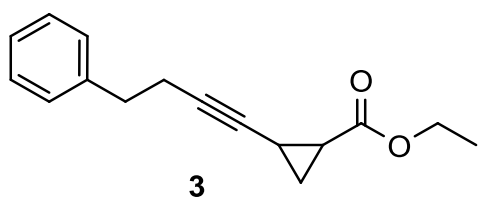

\begin{tabular}{lll} 
Entry $^{[\mathrm{a}]}$ & Light source & Yield $^{[\mathrm{b}]}$ \\
\hline $\mathbf{1}$ & Xe-lamp $(75 \mathrm{~W})$ & $\mathbf{7 9} \%\left(\mathbf{8 1} \%{ }^{[\mathrm{cc}}\right)$ \\
$\mathbf{2}$ & Hg-lamp $(180 \mathrm{~W})$ & $38 \%$ \\
$\mathbf{3}$ & Blue LEDs $(405 \mathrm{~nm})$ & $45 \%$ \\
\hline
\end{tabular}

${ }^{[a]}$ All reactions were performed on $0.50 \mathrm{mmol}$ scale in dry solvents at room temperature under a nitrogen atmosphere; ${ }^{[\mathrm{b}]}$ Yield determined by ${ }^{1} \mathrm{H}$ NMR integration using mesitylene as internal standard; ${ }^{[c]}$ Isolated yield. 


\section{Preparation of the Starting Materials}

\section{General procedure 1 (GP-1): Seyferth-Gilbert homologation.}

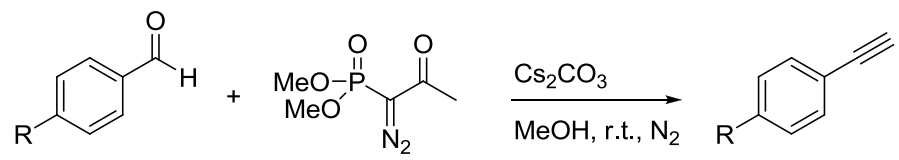

The corresponding aldehyde (1 eq.) was loaded in a dry flask under nitrogen and dissolved in dry $\mathrm{MeOH}$, then $\mathrm{Cs}_{2} \mathrm{CO}_{3}$ (3 - 4 eq.) was added. The Ohira-Bestmann reagent (1.3 - 1.5 eq.) was added dropwise and the reaction mixture was stirred at room temperature until TLC indicated complete conversion. The reaction was then quenched by addition of a saturated $\mathrm{NaHCO}_{3}$ solution and the aqueous layer was extracted three times with DCM. The organic layers were combined, dried over anhydrous $\mathrm{Na}_{2} \mathrm{SO}_{4}$, filtered and concentrated under reduced pressure. The crude product was purified by column chromatography.

\section{General procedure 2 (GP-2): Sonogashira cross-coupling.}
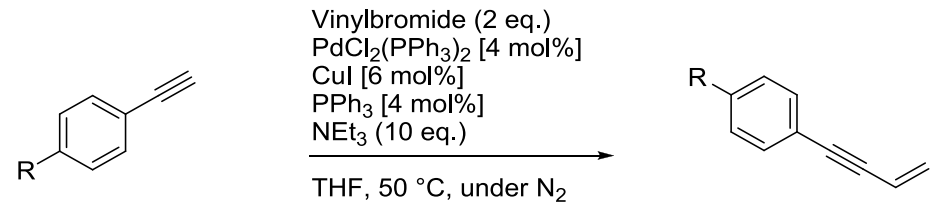

$\mathrm{PdCl}_{2}\left(\mathrm{PPh}_{3}\right)_{2}$ (4 mol\%), Cul (6 mol\%) and $\mathrm{PPh}_{3}(4 \mathrm{~mol} \%)$ were loaded in a dry flask under nitrogen and suspended in dry THF. Dry $\mathrm{NEt}_{3}(10 \mathrm{eq})$, the corresponding alkyne (1 eq.), and vinylbromide (1 M in THF, 2 eq.) were subsequently added and the reaction mixture was stirred at $50^{\circ} \mathrm{C}$ (oil bath) until complete conversion was indicated by TLC or GC. The solvent was removed under reduced pressure, a saturated $\mathrm{NH}_{4} \mathrm{Cl}$ solution was added to the resulting residue and the aqueous layer was extracted three times with DCM. The combined organic layers were dried over anhydrous $\mathrm{Na}_{2} \mathrm{SO}_{4}$, filtered and concentrated under reduced pressure. The crude product was purified by column chromatography.

hex-5-en-3-yn-1-ylbenzene $1^{[2]}$

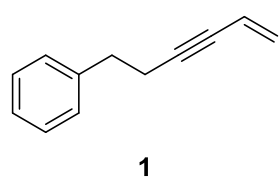

$\mathrm{PdCl}_{2}\left(\mathrm{PPh}_{3}\right)_{2}$ (701.9 mg, $\left.1 \mathrm{mmol}, 5 \mathrm{~mol} \%\right)$ was loaded in a dry schlenk under nitrogen. The schlenk was evacuated for $15 \mathrm{~min}$, then refilled with nitrogen. Dry NEt 3 (8.36 mL, $60 \mathrm{mmol}, 3$ eq.), Cul (266.6 $\mathrm{mg}, 1.4 \mathrm{mmol}, 7 \mathrm{~mol} \%$ ) and vinyl bromide (1 M in THF, $100 \mathrm{~mL}, 100 \mathrm{mmol}, 5$ eq.) were subsequently added. The mixture was stirred at room temperature for 5 minutes, then 4-phenyl-1-butyne $(2.81 \mathrm{~mL}$, $20 \mathrm{mmol}, 1$ eq.) was added. The resulting mixture was stirred overnight at room temperature. The reaction mixture was diluted in $\mathrm{Et}_{2} \mathrm{O}$ and quenched by addition of a saturated $\mathrm{NH}_{4} \mathrm{Cl}$ solution. The aqueous layer was extracted with $\mathrm{Et}_{2} \mathrm{O}$, the combined organic layers were washed with water and brine, dried over anhydrous $\mathrm{Na}_{2} \mathrm{SO}_{4}$, filtered and concentrated under reduced pressure. Purification by 
column chromatography $\left(\mathrm{SiO}_{2}\right.$, petroleum ether) yielded enyne $1(2.58 \mathrm{~g}, 16.54 \mathrm{mmol}, 83 \%)$ as a yellow oil.

${ }^{1} \mathrm{H}$ NMR (400 MHz, $\left.\mathrm{CDCl}_{3}\right), \delta(\mathrm{ppm}):$ 7.32-7.29 (m, $\left.2 \mathrm{H}\right), 7.24-7.20(\mathrm{~m}, 3 \mathrm{H}), 5.78$ (ddt, $J=17.5,11.0$, $2.1 \mathrm{~Hz}, 1 \mathrm{H}$ ), 5.55 (dd, $J=17.5,2.2 \mathrm{~Hz}, 1 \mathrm{H}), 5.39(\mathrm{dd}, J=11.0,2.2 \mathrm{~Hz}, 1 \mathrm{H}), 2.86(\mathrm{t}, J=7.6 \mathrm{~Hz}, 2 \mathrm{H})$, $2.60(\mathrm{dt}, J=7.6,1.7 \mathrm{~Hz}, 2 \mathrm{H}) ;{ }^{13} \mathrm{C}$ NMR $\left(100 \mathrm{MHz}, \mathrm{CDCl}_{3}\right) \delta(\mathrm{ppm}): 140.6,128.4$ (2), 128.3 (2), 126.3, 125.8, 117.5, 90.2, 80.0, 35.1, 21.6; IR (film) $v=3063$ (w), 3028 (w), 2928 (w), $2862(w), 2227$ (w), $1605(\mathrm{~m}), 1496(\mathrm{~m}), 1453(\mathrm{~m}), 1429(\mathrm{~m}), 1411(\mathrm{w}), 1340(\mathrm{w}), 1291(\mathrm{w}), 1260(\mathrm{w}), 1161(\mathrm{w}), 1077(\mathrm{w})$, $1030(\mathrm{w}), 973(\mathrm{~m}), 916(\mathrm{~m}), 835(\mathrm{w}), 748(\mathrm{~m}), 696(\mathrm{~s}), 574(\mathrm{w}), 503(\mathrm{~m}) ; \mathbf{G C} / \mathbf{M S}(\mathrm{El}, 70 \mathrm{eV}): \mathrm{m} / \mathrm{z}(\%)$ : 156 (26), 155 (21), 141 (16), 128 (12), 115 (7), 91 (100), 65 (18), 39 (7); $\boldsymbol{R}_{\mathbf{f}}=0.42$ (petroleum ether).

The analytical data were consistent with those published in the literature ${ }^{[3]}$.

\section{but-3-en-1-yn-1-ylbenzene s1}

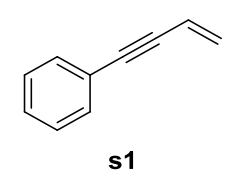

According to GP-2, $\mathrm{PdCl}_{2}\left(\mathrm{PPh}_{3}\right)_{2}$ (280.8 mg, $0.4 \mathrm{mmol}, 4 \mathrm{~mol} \%$ ), Cul (114.3 mg, $0.6 \mathrm{mmol}, 6 \mathrm{~mol} \%$ ), $\mathrm{PPh}_{3}$ (104.9 mg, $0.4 \mathrm{mmol}, 4$ mol\%), dry NEt $3(14 \mathrm{~mL}, 100 \mathrm{mmol}, 10$ eq.), phenylacetylene $(1.1 \mathrm{~mL}, 10$ mmol, 1 eq.), and vinylbromide ( $1 \mathrm{M}$ in THF, $20 \mathrm{~mL}, 20 \mathrm{mmol}, 2$ eq.) were stirred in dry THF (50 mL) under nitrogen at $50{ }^{\circ} \mathrm{C}$ for 1.5 hours. Purification by column chromatography $\left(\mathrm{SiO}_{2}\right.$, pentane) yielded enyne $\mathbf{s} 1(1.05 \mathrm{~g}, 8.21 \mathrm{mmol}, 82 \%)$ as a yellow oil.

${ }^{1} \mathrm{H}$ NMR $\left(400 \mathrm{MHz}, \mathrm{CDCl}_{3}\right), \delta(\mathrm{ppm}): 7.45(\mathrm{~m}, 2 \mathrm{H}), 7.31(\mathrm{~m}, 3 \mathrm{H}), 6.03(\mathrm{dd}, J=17.5,11.1 \mathrm{~Hz}, 1 \mathrm{H})$, $5.74(\mathrm{dd}, J=17.5,2.1 \mathrm{~Hz}, 1 \mathrm{H}), 5.55(\mathrm{dd}, J=11.1,2.1 \mathrm{~Hz}, 1 \mathrm{H}) ;{ }^{13} \mathrm{C}$ NMR $\left(100 \mathrm{MHz}, \mathrm{CDCl}_{3}\right) \delta(\mathrm{ppm})$ : 131.6 (2), 128.3 (2), 128.2, 126.9, 123.2, 117.2, 90.0, 88.0; IR (film) $v=3056$ (w), 3010 (w), 2215 (w), $1843(w), 1663(w), 1605(m), 1489(m), 1442(m), 1411(w), 1291(w), 1262(w), 1176(w), 1069(w)$, 1027 (w), $969(\mathrm{~m}), 916(\mathrm{~m}), 753$ (s), 689 (s), $552(\mathrm{w}), 523(\mathrm{~m}), 498(\mathrm{w})$; GC/MS (El, $70 \mathrm{eV}): \mathrm{m} / \mathrm{z}(\%)$ : 128 (100), 127 (27), 102 (26), 98 (3), 87 (3), 77 (6), 74 (7), 63 (7), 51 (8); $\boldsymbol{R}_{\mathbf{f}}=0.57$ (petroleum ether). The analytical data were consistent with those published in the literature ${ }^{[4]}$.

\section{1-bromo-4-ethynylbenzene $s 2^{[5]}$}

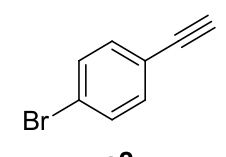

s2

According to GP-1, 4-bromobenzaldehyde (925.0 mg, 5 mmol, 1 eq.), $\mathrm{Cs}_{2} \mathrm{CO}_{3}$ (6.52 g, $20 \mathrm{mmol}, 4$ eq.) and the Ohira-Bestmann reagent ( $976 \mu \mathrm{L}, 6.5 \mathrm{mmol}, 1.3$ eq.) were stirred in dry $\mathrm{MeOH}(35 \mathrm{~mL})$ at room temperature for 15 minutes. The reaction was quenched by addition of water and the aqueous layer was extracted three times with DCM. Purification by flash chromatography $\left(\mathrm{SiO}_{2}\right.$, petroleum ether) yielded alkyne s2 (585 mg, $3.23 \mathrm{mmol}, 65 \%$ ) as an off-white solid.

${ }^{1} \mathrm{H}$ NMR (400 MHz, CDCl $), \delta(\mathrm{ppm}): 7.46$ (d, $\left.J=8.5 \mathrm{~Hz}, 2 \mathrm{H}\right), 7.35$ (m, $\left.J=8.5 \mathrm{~Hz}, 2 \mathrm{H}\right), 3.12(\mathrm{~s}, 1 \mathrm{H})$; ${ }^{13} \mathrm{C}$ NMR (100 MHz, $\left.\mathrm{CDCl}_{3}\right) \delta(\mathrm{ppm}): 133.5$ (2), 131.6 (2), 123.1, 121.1, 82.6, 78.3; IR (film) $v=3264$ (m), $2109(w), 1902(w), 1647(w), 1585(m), 1484(s) 1468(m), 1396(m), 1235(w), 1096(w), 1069$ 
(s), 1011 (s), 907 (s), 821 (s), 770 (w), 733 (s), $671(\mathrm{~m}), 633(\mathrm{~m}), 619(\mathrm{~m}), 544$ (w), 524 (s); MS (El,

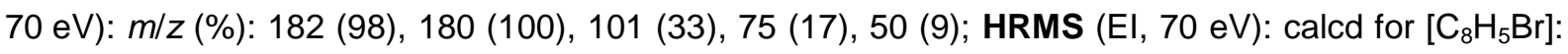
179.9575; found: $179.9574 ; \boldsymbol{R}_{\mathrm{f}}=0.74$ (petroleum ether/ethyl acetate $10 / 1$ ); Melting point: $66^{\circ} \mathrm{C}$.

The analytical data were consistent with those published in the literature ${ }^{[6]}$.

\section{1-bromo-4-(but-3-en-1-yn-1-yl)benzene $s 3^{[7]}$}

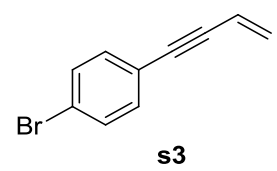

$\mathrm{PdCl}_{2}\left(\mathrm{PPh}_{3}\right)_{2}$ (44.2 mg, $\left.0.06 \mathrm{mmol}, 2 \mathrm{~mol} \%\right)$, Cul (12.0 mg, $\left.0.06 \mathrm{mmol}, 2 \mathrm{~mol} \%\right), \mathrm{PPh}_{3}$ (33.0 mg, 0.13 mmol, 4 mol\%), dry $\mathrm{NEt}_{3}(1 \mathrm{~mL}, 7.2 \mathrm{mmol}, 2.3$ eq.), 1-bromo-4-ethynylbenzene s2 (570.0 mg, 3.15 mmol, 1 eq.), and vinylbromide ( $1 \mathrm{M}$ in THF, $3.78 \mathrm{~mL}, 3.78 \mathrm{mmol}, 1.2$ eq.) were stirred in dry THF (5 $\mathrm{mL}$ ) under nitrogen at room temperature overnight. An additional amount of vinylbromide (1 M in THF, $1.6 \mathrm{~mL}, 1.6 \mathrm{mmol}, 0.5$ eq.) was added and the reaction mixture was stirred at $50^{\circ} \mathrm{C}$ (oil bath) for 4.5 hours. Purification by column chromatography $\left(\mathrm{SiO}_{2}\right.$, pentane) yielded enyne s3 $(403 \mathrm{mg}, 3.15 \mathrm{mmol}$, quantitative) as an orange oil.

${ }^{1} \mathrm{H}$ NMR (300 MHz, $\mathrm{CDCl}_{3}$ ), $\delta$ (ppm): 7.45 (d, $J=8.7 \mathrm{~Hz}, 2 \mathrm{H}$ ), 7.30 (d, $J=8.6 \mathrm{~Hz}, 2 \mathrm{H}$ ), 5.99 (dd, $J=$ 17.5, $11.1 \mathrm{~Hz}, 1 \mathrm{H}), 5.74(\mathrm{~d}, J=17.5,2.1 \mathrm{~Hz}, 1 \mathrm{H}), 5.57$ (d, $J=11.1,2.1 \mathrm{~Hz}, 1 \mathrm{H}) ;{ }^{13} \mathrm{C}$ NMR $(75 \mathrm{MHz}$, $\mathrm{CDCl}_{3}$ ) $\delta$ (ppm): 133.0 (2), 131.6 (2), 127.4, 122.5, 122.1, 116.9, 89.2, 88.9; IR (film) $v=3009$ (w), 2214 (w), 2170 (w), 1901 (w), 1839 (w), 1646 (w), 1607 (w), 1582 (m), 1484 (s), 1412 (w), 1394 (m), $1290(\mathrm{w}), 1265(\mathrm{~m}), 1095(\mathrm{w}), 1069(\mathrm{~s}), 1011(\mathrm{~s}), 968(\mathrm{~m}), 920(\mathrm{~m}), 822(\mathrm{~s}), 727(\mathrm{~m}), 519(\mathrm{~m})$; MS (El, $70 \mathrm{eV}):$ m/z (\%): 208 (98), 206 (100), 182 (5), 180 (5), 127 (62), 101 (12), 75 (12), 63 (13), 50 (7); HRMS (El, $70 \mathrm{eV}$ ): calcd for $\left[\mathrm{C}_{10} \mathrm{H}_{7} \mathrm{Br}\right.$ ]: 205.9731; found: 205.9735; $\boldsymbol{R}_{\mathbf{f}}=0.8$ (petroleum ether/ethyl acetate $10 / 1$ ).

The analytical data were consistent with those published in the literature ${ }^{[8]}$.

\section{1-ethynyl-4-methoxybenzene $54^{[9]}$}

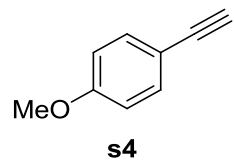

According to GP-1, $p$-anisaldehyde ( $608 \mu \mathrm{L}, 5 \mathrm{mmol}, 1$ eq.), $\mathrm{Cs}_{2} \mathrm{CO}_{3}(4.89 \mathrm{~g}, 15 \mathrm{mmol}, 3$ eq.) and the Ohira-Bestmann reagent $(1.13 \mathrm{~mL}, 7.5 \mathrm{mmol}, 1.5$ eq.) were stirred in dry $\mathrm{MeOH}(30 \mathrm{~mL})$ at room temperature for 6 hours, then an additional amount of the Ohira-Bestmann reagent $(150 \mu \mathrm{L}, 1 \mathrm{mmol}$, 0.2 eq.) was added and the reaction mixture was stirred overnight at room temperature. Purification by column chromatography $\left(\mathrm{SiO}_{2}\right.$, pentane/ $\left.\mathrm{Et}_{2} \mathrm{O} 20 / 1\right)$ yielded alkyne s4 (552 mg, $\left.4.17 \mathrm{mmol}, 83 \%\right)$ as a colorless oil.

${ }^{1} \mathrm{H}$ NMR $\left(400 \mathrm{MHz}, \mathrm{CDCl}_{3}\right), \delta(\mathrm{ppm}): 7.43(\mathrm{~d}, J=8.9 \mathrm{~Hz}, 2 \mathrm{H}), 6.84(\mathrm{~d}, J=8.9 \mathrm{~Hz}, 2 \mathrm{H}), 3.81(\mathrm{~s}, 3 \mathrm{H})$, 2.99 (s, $1 \mathrm{H}) ;{ }^{13} \mathrm{C}$ NMR $\left(100 \mathrm{MHz}, \mathrm{CDCl}_{3}\right) \delta$ (ppm): 160.0, 133.6 (2), 114.2, 113.9 (2), 83.7, 75.8, 55.3; IR (film) v = 3285 (m), 3005 (w), 2960 (w), 2838 (w), 2106 (w), 1605 (s), 1571 (w), 1505 (s), 1465 (m), $1441(\mathrm{~m}), 1290$ (s), 1246 (s), 1170 (s), 1108 (m), 1030 (s), 831 (s), $811(\mathrm{~m}), 685(\mathrm{~m}), 660$ (m), 641 
(m), $609(\mathrm{~m}), 537(\mathrm{~m}), 487$ (w), 450 (w); MS (El, $70 \mathrm{eV}): \mathrm{m} / \mathrm{z}(\%): 132$ (100), 117 (21), 89 (26), $75(4)$, 63 (10); HRMS (El, $70 \mathrm{eV}$ ): calcd for $\left[\mathrm{C}_{9} \mathrm{H}_{8} \mathrm{O}\right]: 132.0575$; found: 132.0576; $\boldsymbol{R}_{\mathbf{f}}=0.58$ (petroleum ether/ethyl acetate 10/1).

The analytical data were consistent with those published in the literature ${ }^{[10]}$.

\section{1-(but-3-en-1-yn-1-yl)-4-methoxybenzene $s 5^{[7]}$}

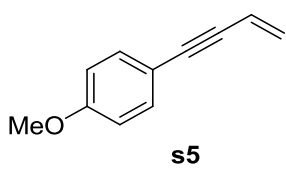

$\mathrm{PdCl}_{2}\left(\mathrm{PPh}_{3}\right)_{2}(57.6 \mathrm{mg}, 0.082 \mathrm{mmol}, 2 \mathrm{~mol} \%)$, Cul (15.6 mg, $\left.0.082 \mathrm{mmol}, 2 \mathrm{~mol} \%\right), \mathrm{PPh}_{3}(43.0 \mathrm{mg}$, $0.082 \mathrm{mmol}, 4 \mathrm{~mol} \%)$, dry NEt ${ }_{3}(1.3 \mathrm{~mL}, 9.43 \mathrm{mmol}, 2.3$ eq.), 1-ethynyl-4-methoxybenzene s4 (543.0 $\mathrm{mg}, 4.1 \mathrm{mmol}$, 1 eq.), and vinylbromide (1 $\mathrm{M}$ in THF, $7.0 \mathrm{~mL}, 7.0 \mathrm{mmol}, 1.7$ eq.) were stirred in dry THF (10 mL) under nitrogen at $50{ }^{\circ} \mathrm{C}$ (oil bath) overnight. Additional amounts of $\mathrm{PdCl}_{2}\left(\mathrm{PPh}_{3}\right)_{2}(57.6$ mg, $0.082 \mathrm{mmol}, 2 \mathrm{~mol} \%)$, Cul (31.2 mg, $0.16 \mathrm{mmol}, 4 \mathrm{~mol} \%$ ) and dry NEt $\mathrm{N}_{3}(5.7 \mathrm{~mL}, 41 \mathrm{mmol}, 10 \mathrm{eq}$.) were added and the reaction mixture was stirred at $50{ }^{\circ} \mathrm{C}$ for 3.5 hours. Purification by column chromatography $\left(\mathrm{SiO}_{2}\right.$, pentane/ $\mathrm{Et}_{2} \mathrm{O} 40 / 1$ to $20 / 1$ ) yielded enyne s5 (505 mg, $3.19 \mathrm{mmol}, 78 \%$ ) as an orange oil.

${ }^{1} \mathrm{H}$ NMR $\left(400 \mathrm{MHz}, \mathrm{CDCl}_{3}\right), \delta(\mathrm{ppm}): 7.39$ (d, $\left.J=8.9 \mathrm{~Hz}, 2 \mathrm{H}\right), 6.85$ (d, $\left.J=8.9 \mathrm{~Hz}, 2 \mathrm{H}\right), 6.02$ (dd, $J=$ 17.5, $11.1 \mathrm{~Hz}, 1 \mathrm{H}), 5.70(\mathrm{dd}, J=17.5,2.1 \mathrm{~Hz}, 1 \mathrm{H}), 5.50(\mathrm{dd}, J=11.1,2.1 \mathrm{~Hz}, 1 \mathrm{H}), 3.80(\mathrm{~s}, 3 \mathrm{H}) ;{ }^{13} \mathrm{C}$ NMR (100 MHz, $\left.\mathrm{CDCl}_{3}\right) \delta(\mathrm{ppm}): 159.7,133.0$ (2), 126.0, 117.4, 115.3, 114.0 (2), 90.0, 86.9, 55.2; IR (film) $v=3006(w), 2957(w), 2934(w), 2836(w), 2542(w), 2215(w), 2182(w), 2045(w), 1889(w)$, 1599 (s), 1562 (m), 1506 (s), 1463 (m), 1441 (m), 1414 (w), 1290 (s), 1243 (s), 1171 (s), 1106 (m), $1078(\mathrm{~m}), 1029(\mathrm{~s}), 970(\mathrm{~m}), 915(\mathrm{~m}), 828(\mathrm{~s}), 795(\mathrm{~m}), 674(\mathrm{w}), 648(\mathrm{w}), 634(\mathrm{~m}), 532(\mathrm{~s}), 493(\mathrm{w})$; MS (El, $70 \mathrm{eV}$ ): m/z (\%): 158 (100), 143 (27), 115 (21), 89 (4), 79 (3), 63 (4); HRMS (EI, $70 \mathrm{eV)}$ : calcd for $\left[\mathrm{C}_{11} \mathrm{H}_{10} \mathrm{O}\right]: 158.0732$, found: $158.0731 ; \boldsymbol{R}_{\mathbf{f}}=0.62$ (petroleum ether/ethyl acetate 10/1).

The analytical data were consistent with those published in the literature ${ }^{[11]}$.

\section{4-(but-3-en-1-yn-1-yl)-N,N-dimethylaniline $s 6^{[7]}$}

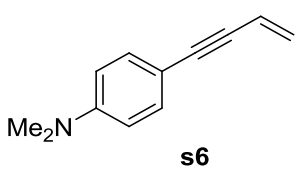

$\mathrm{PdCl}_{2}\left(\mathrm{PPh}_{3}\right)_{2}$ (70.2 mg, $\left.0.1 \mathrm{mmol}, 2 \mathrm{~mol} \%\right)$, Cul (19 mg, $\left.0.1 \mathrm{mmol}, 2 \mathrm{~mol} \%\right), \mathrm{PPh}_{3}(52.5 \mathrm{mg}, 0.2 \mathrm{mmol}$, 2 mol\%), dry $\mathrm{NEt}_{3}(1.6 \mathrm{~mL}, 11.5 \mathrm{mmol}, 2.3$ eq.), 4-ethynyl- $N, N$-dimethylamine (726.0 mg, $5 \mathrm{mmol}, 1$ eq.) and vinylbromide (1 $\mathrm{M}$ in THF, $6.0 \mathrm{~mL}, 6.0 \mathrm{mmol}, 1.2$ eq.) were stirred in dry THF (10 mL) under nitrogen at room temperature overnight. An extra amount of vinylbromide (1 M in THF, 7.5 mL, 7.5 $\mathrm{mmol}, 1.5$ eq.) was added and the reaction mixture was stirred at $50^{\circ} \mathrm{C}$ (oil bath) overnight. Additional amounts of $\mathrm{PdCl}_{2}\left(\mathrm{PPh}_{3}\right)_{2}(70.2 \mathrm{mg}, 0.1 \mathrm{mmol}, 2 \mathrm{~mol} \%)$, Cul $(38.1 \mathrm{mg}, 0.2 \mathrm{mmol}, 4 \mathrm{~mol} \%)$ and dry $\mathrm{NEt}_{3}$ (6.96 mL, $50 \mathrm{mmol}, 10$ eq.) were added and the reaction mixture was stirred at $50{ }^{\circ} \mathrm{C}$ for 4 hours. Purification by column chromatography $\left(\mathrm{SiO}_{2}\right.$, pentane/ $\mathrm{Et}_{2} \mathrm{O} 40 / 1$ to $\left.5 / 1\right)$ yielded enyne s6 (663 mg, $3.87 \mathrm{mmol}, 77 \%$ ) as a yellow solid. 
${ }^{1} \mathrm{H}$ NMR $\left(400 \mathrm{MHz}, \mathrm{CDCl}_{3}\right), \delta(\mathrm{ppm}): 7.34(\mathrm{~d}, J=8.9 \mathrm{~Hz}, 2 \mathrm{H}), 6.64(\mathrm{~d}, J=8.9 \mathrm{~Hz}, 2 \mathrm{H}), 6.04$ (dd, $J=$ 17.5, $11.1 \mathrm{~Hz}, 1 \mathrm{H}$ ), 5.67 (dd, $J=17.5,2.1 \mathrm{~Hz}, 1 \mathrm{H}), 5.21$ (dd, $J=11.1,2.2 \mathrm{~Hz}, 1 \mathrm{H}), 2.98(\mathrm{~s}, 6 \mathrm{H}) ;{ }^{13} \mathrm{C}$ NMR (100 MHz, $\left.\mathrm{CDCl}_{3}\right) \delta$ (ppm): 149.1, 131.7 (2), 123.8, 116.7, 110.7 (2), 108.8, 90.3, 85.2, 39.1 (2); IR (film) $v=3094$ (w), 3041 (w), 3003 (w), $2891(\mathrm{~m}), 2859(\mathrm{~m}), 2804(\mathrm{~m}), 2215(\mathrm{~m}), 2182(\mathrm{~m}), 1611$ (s), $1596(\mathrm{~s}), 1518(\mathrm{~s}), 1481(\mathrm{~m}), 1444(\mathrm{~m}), 1411(\mathrm{w}), 1357(\mathrm{~s}), 1290(\mathrm{w}), 1225(\mathrm{~m}), 1189(\mathrm{~s}), 1169$ (m), $1126(\mathrm{~s}), 1078(\mathrm{~m}), 1063(\mathrm{~m}), 970(\mathrm{~m}), 947(\mathrm{~m}), 911(\mathrm{~m}), 815(\mathrm{~s}), 673(\mathrm{w}), 531(\mathrm{~m}), 511(\mathrm{~m})$; MS (El, $70 \mathrm{eV}): \mathrm{m} / z$ (\%): 171 (100), 155 (13), 127 (8), 101 (2), 85 (6), 77 (3), 63 (2); HRMS (El, $70 \mathrm{eV):}$ calcd for $\left[\mathrm{C}_{12} \mathrm{H}_{13} \mathrm{~N}\right]: 171.1048$; found: $171.1045 ; \boldsymbol{R}_{\mathfrak{f}}=0.5$ (petroleum ether/ethyl acetate 10/1); Melting point: $53^{\circ} \mathrm{C}$.

\section{1-ethynyl-4-methylbenzene $s 7^{[0]}$}

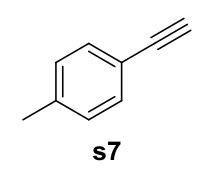

According to GP-1, p-tolualdehyde (590 $\mathrm{LL}, 5 \mathrm{mmol}, 1$ eq.), $\mathrm{Cs}_{2} \mathrm{CO}_{3}(4.89 \mathrm{~g}, 15 \mathrm{mmol}, 3$ eq.) and the Ohira-Bestmann reagent $(1.13 \mathrm{~mL}, 7.5 \mathrm{mmol}, 1.5$ eq.) were stirred in dry $\mathrm{MeOH}(30 \mathrm{~mL})$ at room temperature for 1 hour. Purification by flash chromatography $\left(\mathrm{SiO}_{2}\right.$, pentane) yielded alkyne s7 (260 $\mathrm{mg}, 2.23 \mathrm{mmol}, 45 \%$ ) as a colorless oil.

${ }^{1} \mathrm{H}$ NMR $\left(300 \mathrm{MHz}, \mathrm{CDCl}_{3}\right), \delta(\mathrm{ppm}): 7.39(\mathrm{~d}, J=8.1 \mathrm{~Hz}, 2 \mathrm{H}), 7.13(\mathrm{~d}, J=8.0 \mathrm{~Hz}, 2 \mathrm{H}), 3.03(\mathrm{~s}, 1 \mathrm{H})$, 2.36 (s, $3 \mathrm{H}$ ); ${ }^{13} \mathrm{C}$ NMR $\left(75 \mathrm{MHz}, \mathrm{CDCl}_{3}\right) \delta$ (ppm): 138.9, 132.0 (2), 129.1 (2), 119.0, 83.8, 76.4, 21.5; IR (film) $v=3290$ (m), 3029 (w), 2922 (w), 2856 (w), 2108 (w), 1908 (w), 1605 (w), 1507 (m), 1449 (w), $1215(w), 1175(w), 1106(w), 1039(w), 1021(w), 945(w), 815(s), 654(m), 643(m), 608(s), 528$ (s), $515(\mathrm{~m}), 410(\mathrm{~m})$; MS (El, $70 \mathrm{eV}): \mathrm{m} / z(\%): 116$ (93), 115 (100), 89 (7), 76 (3), 74 (3) , 63 (7); $\boldsymbol{R}_{\mathrm{f}}=$ 0.5 (petroleum ether).

The analytical data were consistent with those published in the literature ${ }^{[12]}$.

1-(but-3-en-1-yn-1-yl)-4-methylbenzene $s 8^{[9]}$

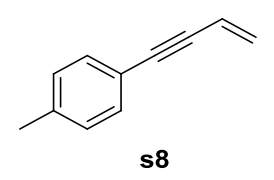

According to GP-2, $\mathrm{PdCl}_{2}\left(\mathrm{PPh}_{3}\right)_{2}(59.0 \mathrm{mg}, 0.084 \mathrm{mmol}, 4 \mathrm{~mol} \%)$, Cul (24.0 mg, $\left.0.13 \mathrm{mmol}, 6 \mathrm{~mol} \%\right)$, $\mathrm{PPh}_{3}$ (22.0 mg, 0.084 mmol, 4 mol\%), dry NEt 3 (2.9 mL, $21 \mathrm{mmol}, 10$ eq.), 1-ethynyl-4-methylbenzene s7 (244.0 mg, $2.1 \mathrm{mmol}, 1$ eq.), and vinylbromide ( $1 \mathrm{M}$ in THF, $4.2 \mathrm{~mL}, 4.2 \mathrm{mmol}, 2$ eq.) were stirred in dry THF $(10 \mathrm{~mL})$ under nitrogen at $50{ }^{\circ} \mathrm{C}$ for 3.5 hours. Purification by column chromatography $\left(\mathrm{SiO}_{2}\right.$, pentane) yielded enyne $\mathbf{s} 8$ (227 $\mathrm{mg}, 1.59 \mathrm{mmol}, 76 \%)$ as a colorless oil.

${ }^{1} \mathbf{H}$ NMR $\left(400 \mathrm{MHz}, \mathrm{CDCl}_{3}\right), \delta(\mathrm{ppm}): 7.34(\mathrm{~d}, J=8.1,2 \mathrm{H}), 7.12(\mathrm{~d}, J=7.8 \mathrm{~Hz}, 2 \mathrm{H}), 6.01$ (dd, $J=$ $17.5,11.1 \mathrm{~Hz}, 1 \mathrm{H}$ ), 5.71 (dd, $J=17.5,2.1 \mathrm{~Hz}, 1 \mathrm{H}), 5.51$ (dd, $J=11.1,2.1 \mathrm{~Hz}, 1 \mathrm{H}), 2.35(\mathrm{~s}, 3 \mathrm{H}) ;{ }^{13} \mathrm{C}$ NMR (100 MHz, $\left.\mathrm{CDCl}_{3}\right) \delta$ (ppm): 138.4, 131.5 (2), 129.1 (2), 126.4, 120.1, 117.3, 90.2, 87.5, 21.5; IR (film) $v=3008(w), 2921(w), 2216(w), 1841(w), 1602(m), 1508(m), 1448(w), 1410(w), 1291(w)$, $1265(w), 1180(w), 1106(w), 1077(w), 1020(w), 969(m), 917(m), 814(s), 708(w), 674(w), 525$ 
(m), 496 (w), 414 (w); MS (El, $70 \mathrm{eV}):$ m/z (\%): 142 (100), 115 (25), 91 (5), 71 (4), 63 (6); HRMS (El, $70 \mathrm{eV}$ ): calcd for $\left[\mathrm{C}_{11} \mathrm{H}_{10}\right]$ : 142.0783 ; found: $142.0783 ; \boldsymbol{R}_{\mathbf{f}}=0.5$ (petroleum ether).

The analytical data were consistent with those published in the literature ${ }^{[13]}$.

\section{4-ethynylbenzonitrile $s 9^{[5]}$}

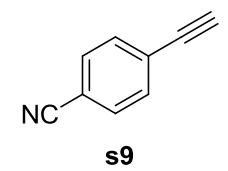

According to GP-1, 4-formylbenzonitrile (655.7 mg, 5 mmol, 1 eq.), $\mathrm{Cs}_{2} \mathrm{CO}_{3}$ (6.52 g, 20 mmol, 4 eq.) and the Ohira-Bestmann reagent (976 $\mathrm{LL}, 6.5 \mathrm{mmol}, 1.3$ eq.) were stirred in dry $\mathrm{MeOH}(35 \mathrm{~mL})$ at room temperature for 2 hours. The reaction was quenched by addition of water and the aqueous layer was extracted three times with DCM. Purification by column chromatography $\left(\mathrm{SiO}_{2}\right.$, pentane/ $\mathrm{Et}_{2} \mathrm{O}$ 10/1) yielded alkyne s9 (490 mg, $3.85 \mathrm{mmol}, 77 \%$ ) as colorless needles.

${ }^{1} \mathrm{H}$ NMR $\left(400 \mathrm{MHz}, \mathrm{CDCl}_{3}\right), \delta(\mathrm{ppm}): 7.62(\mathrm{~d}, J=8.6,2 \mathrm{H}), 7.57(\mathrm{~d}, J=8.5 \mathrm{~Hz}, 2 \mathrm{H}), 3.30(\mathrm{~s}, 1 \mathrm{H}) ;{ }^{13} \mathrm{C}$ NMR (100 MHz, $\mathrm{CDCl}_{3}$ ) $\delta$ (ppm): 132.7 (2), 132.0 (2), 127.0, 118.2, 112.4, 81.9, 81.5; IR (film) $v=$ $3235(\mathrm{~s}), 2227(\mathrm{~m}), 2166(\mathrm{w}), 2102(\mathrm{w}), 1976(\mathrm{w}), 1602(\mathrm{~m}), 1498(\mathrm{~m}), 1407(\mathrm{w}), 1271(\mathrm{w}), 1175(\mathrm{w})$, 838 (s), 730 (m), 685 (m), 555 (s), 506 (w); MS (El, 70 eV): m/z (\%): 127 (100), 100 (10), 74 (6), 63 (3), 50 (7); HRMS (El, $70 \mathrm{eV}$ ): calcd for $\left[\mathrm{C}_{9} \mathrm{H}_{5} \mathrm{~N}\right]$ : 127.0422; found: 127.0424; $\boldsymbol{R}_{\mathrm{f}}=0.47$ (petroleum ether/ethyl acetate $10 / 1)$; Melting point: $157^{\circ} \mathrm{C}$.

The analytical data were consistent with those published in the literature ${ }^{[12]}$.

\section{4-(but-3-en-1-yn-1-yl)benzonitrile s10}

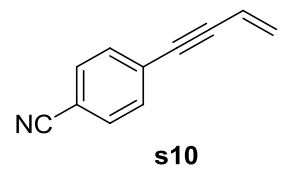

According to GP-2, $\mathrm{PdCl}_{2}\left(\mathrm{PPh}_{3}\right)_{2}$ (106.7 mg, $0.15 \mathrm{mmol}, 4 \mathrm{~mol} \%$ ), Cul (43.4 mg, $0.23 \mathrm{mmol}, 6 \mathrm{~mol} \%$ ), $\mathrm{PPh}_{3}$ (39.9 mg, $0.15 \mathrm{mmol}, 4 \mathrm{~mol} \%$ ), dry $\mathrm{NEt}_{3}(5.3 \mathrm{~mL}, 38 \mathrm{mmol}, 10$ eq.), 4-ethynylbenzonitrile s9 (483.0 mg, $3.8 \mathrm{mmol}, 1$ eq.), and vinylbromide ( $1 \mathrm{M}$ in THF, $7.6 \mathrm{~mL}, 7.6 \mathrm{mmol}, 2$ eq.) were stirred in dry THF $(10 \mathrm{~mL})$ under nitrogen at $50{ }^{\circ} \mathrm{C}$ for 5 hours. Purification by column chromatography $\left(\mathrm{SiO}_{2}\right.$, pentane/ $\left.\mathrm{Et}_{2} \mathrm{O} 20 / 1\right)$ yielded enyne $\mathbf{s} 10(492 \mathrm{mg}, 3.21 \mathrm{mmol}, 85 \%)$ as a yellow solid.

${ }^{1} \mathrm{H}$ NMR (400 MHz, $\mathrm{CDCl}_{3}$ ), $\delta(\mathrm{ppm}): 7.59$ (d, $\left.J=8.4 \mathrm{~Hz}, 2 \mathrm{H}\right), 7.50$ (d, $\left.J=8.4 \mathrm{~Hz}, 2 \mathrm{H}\right), 6.02$ (dd, $J=$ 17.6, $11.2 \mathrm{~Hz}, 1 \mathrm{H}), 5.80$ (dd, $J=17.6,1.9 \mathrm{~Hz}, 1 \mathrm{H}), 5.63(\mathrm{~d}, J=11.2,1.9 \mathrm{~Hz}, 1 \mathrm{H}) ;{ }^{13} \mathrm{C}$ NMR $(100$ $\left.\mathrm{MHz}, \mathrm{CDCl}_{3}\right) \delta$ (ppm): 132.1 (2), 132.0 (2), 128.7, 128.1, 118.4, 116.5, 111.6, 92.3, 88.2; IR (film) $v=$ $3012(w), 2354(w), 2220(m), 2187(w), 1925(w), 1861(w), 1796(w), 1722(w), 1675(w), 1605(m)$, $1514(\mathrm{w}), 1499(\mathrm{~m}), 1436(\mathrm{w}), 1405(\mathrm{~m}), 1290(\mathrm{~m}), 1270(\mathrm{~m}), 1193(\mathrm{~m}), 1178(\mathrm{~m}), 1106(\mathrm{~m}), 1018(\mathrm{w})$, 970 (m), 925 (m), 909 (s), 834 (s), 732 (s), 650 (w), 552 (s), 481 (w); MS (El, 70 eV): m/z (\%); 153

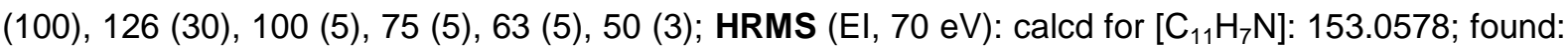
$153.0577 ; \boldsymbol{R}_{\mathfrak{f}}=0.55$ (petroleum ether/ethyl acetate 10/1); Melting point: $65^{\circ} \mathrm{C}$.

The analytical data were consistent with those published in the literature ${ }^{[7]}$. 


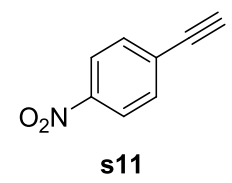

4-Nitrobenzaldehyde ( $378 \mathrm{mg}, 2.5 \mathrm{mmol}, 1$ eq.), $\mathrm{K}_{2} \mathrm{CO}_{3}(691 \mathrm{mg}, 5 \mathrm{mmol}, 2$ eq.) and the OhiraBestmann reagent $(563 \mu \mathrm{L}, 3.75 \mathrm{mmol}, 1.5$ eq.) were stirred in dry $\mathrm{MeOH}(15 \mathrm{~mL})$ at room temperature for 30 minutes. Purification by column chromatography $\left(\mathrm{SiO}_{2}\right.$, pentane/ $\left.\mathrm{Et}_{2} \mathrm{O} 20 / 1\right)$ yielded alkyne s11 (349 mg, $2.37 \mathrm{mmol}, 95 \%$ ) as a yellow solid.

${ }^{1} \mathbf{H}$ NMR (400 MHz, $\mathrm{CDCl}_{3}$ ), $\delta(\mathrm{ppm}): 8.19(\mathrm{~d}, J=8.9,5.5 \mathrm{~Hz}, 2 \mathrm{H}), 7.64(\mathrm{~d}, J=8.9 \mathrm{~Hz}, 2 \mathrm{H}$ ), 3.35 (s, $1 \mathrm{H}) ;{ }^{13} \mathrm{C}$ NMR (100 MHz, $\left.\mathrm{CDCl}_{3}\right) \delta$ (ppm): 147.6, 133.0 (2), 128.9, 123.5 (2), 82.3, 81.6; IR (film) $v=$ $3299(w), 3249(m), 3105(w), 2104(w), 1681(w), 1592(m), 1508(s), 1402(w) 1372(w) ; 1340(s)$, $1311(\mathrm{~m}), 1287(\mathrm{~m}), 1176(\mathrm{w}), 1105(\mathrm{~m}), 965(\mathrm{w}), 854(\mathrm{~s}), 771(\mathrm{w}), 750(\mathrm{~m}), 693(\mathrm{~m}), 677(\mathrm{~m}) 573(\mathrm{w})$, 518 (w), 496 (w); MS (El, $70 \mathrm{eV}): \mathrm{m} / \mathrm{z}(\%): 147$ (100), 117 (22), 101 (53), 89 (18), 75 (37), 63 (6), 51 (15); HRMS (El, $70 \mathrm{eV}$ ): calcd for $\left[\mathrm{C}_{8} \mathrm{H}_{5} \mathrm{NO}_{2}\right]$ : 147.0320; found: 147.0322; $\boldsymbol{R}_{\mathbf{f}}=0.57$ (petroleum ether/ethyl acetate 10/1); Melting point: $148^{\circ} \mathrm{C}$.

The analytical data were consistent with those published in the literature ${ }^{[14]}$.

\section{1-(but-3-en-1-yn-1-yl)-4-nitrobenzene s12}

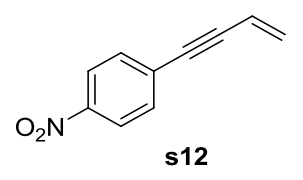

According to GP-2, $\mathrm{PdCl}_{2}\left(\mathrm{PPh}_{3}\right)_{2}(66.3 \mathrm{mg}, 0.094 \mathrm{mmol}, 4 \mathrm{~mol} \%)$, Cul (27.0 mg, $\left.0.14 \mathrm{mmol}, 6 \mathrm{~mol} \%\right)$, $\mathrm{PPh}_{3}$ (24.8 mg, $0.094 \mathrm{mmol}, 4 \mathrm{~mol} \%$ ), dry NEt 3 (3.3 mL, $23.6 \mathrm{mmol}, 10$ eq.), 1-ethynyl-4-nitrobenzene s11 (347.0 mg, $2.36 \mathrm{mmol}, 1$ eq.) and vinylbromide ( $1 \mathrm{M}$ in THF, $4.7 \mathrm{~mL}, 4.7 \mathrm{mmol}, 2$ eq.) were stirred in dry THF $(10 \mathrm{~mL})$ under nitrogen at $50{ }^{\circ} \mathrm{C}$ for 5 hours. Purification by column chromatography $\left(\mathrm{SiO}_{2}\right.$, pentane/ $\left.\mathrm{Et}_{2} \mathrm{O} 20 / 1\right)$ yielded enyne $\mathbf{s 1 2}(327 \mathrm{mg}, 1.89 \mathrm{mmol}, 80 \%)$ as a yellow solid.

${ }^{1} \mathrm{H}$ NMR $\left(400 \mathrm{MHz}, \mathrm{CDCl}_{3}\right), \delta(\mathrm{ppm}): 8.19(\mathrm{~d}, J=8.9 \mathrm{~Hz}, 2 \mathrm{H}), 7.57(\mathrm{~m}, J=5.0 \mathrm{~Hz}, 2 \mathrm{H}), 6.04$ (dd, $J=$ 17.5, $11.2 \mathrm{~Hz}, 1 \mathrm{H}$ ), 5.83 (dd, $J=17.5,2.0 \mathrm{~Hz}, 1 \mathrm{H}), 5.67$ (dd, $J=11.2,2.0 \mathrm{~Hz}, 1 \mathrm{H}) ;{ }^{13} \mathrm{C}$ NMR $(100$ $\left.\mathrm{MHz} \mathrm{CDCl}_{3}\right) \delta$ (ppm): 147.0, 132.3 (2), 130.1, 129.0, 123.6 (2), 116.5, 93.2, 88.0; IR (film) $v=3105$ (w), $2930(w), 2840(w), 2220(w), 1925(w), 1879(w), 1569(s), 1513(s), 1491(s), 1401(w), 1374$ (w), $1340(\mathrm{~s}), 1308(\mathrm{~m}), 1285(\mathrm{~m}), 1271(\mathrm{~m}), 1175(\mathrm{w}), 1106(\mathrm{~m}), 1013(\mathrm{w}), 974(\mathrm{~m}), 936(\mathrm{~m}), 908(\mathrm{~s})$, 851 (s), 748 (s), 730 (s), 685 (s), 649 (w), 575 (w), 539 (w), $514(\mathrm{~m}), 468$ (m); MS (El, 70 eV): m/z (\%): 173 (100), 143 (15), 127 (24), 115 (16), 101 (11), 89 (6), 77 (13), 63 (6), 51 (8); HRMS (El, 70 eV): calcd for $\left[\mathrm{C}_{10} \mathrm{H}_{7} \mathrm{NO}_{2}\right]$ : 173.0477; found: 173.0476; $\boldsymbol{R}_{\mathbf{f}}=0.62$ (petroleum ether/ethyl acetate 10/1); Melting point: $77^{\circ} \mathrm{C}$.

The analytical data were consistent with those published in the literature ${ }^{[15]}$. 


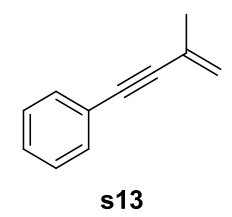

$\mathrm{PdCl}_{2}\left(\mathrm{PPh}_{3}\right)_{2}(70.2 \mathrm{mg}, 0.1 \mathrm{mmol}, 2 \mathrm{~mol} \%)$, Cul $(47.6 \mathrm{mg}, 0.25 \mathrm{mmol}, 5 \mathrm{~mol} \%)$, dry THF $(5 \mathrm{~mL})$ and dry $\mathrm{NEt}_{3}(5 \mathrm{~mL})$ were sequentially loaded in a Schlenk under nitrogen. 2-Bromopropene $(444 \mu \mathrm{L}, 5$ mmol, 1 eq.) was added, followed by a dropwise addition of phenylacetylene (604 $\mu \mathrm{L}, 5.5 \mathrm{mmol}, 1.1$ eq.). The reaction mixture was stirred at $50^{\circ} \mathrm{C}$ (oil bath) for 2 days. The reaction was quenched by addition of a saturated $\mathrm{NH}_{4} \mathrm{Cl}$ solution and extracted three times with EtOAc. The combined organic layers were dried over anhydrous $\mathrm{Na}_{2} \mathrm{SO}_{4}$, filtered and concentrated under reduced pressure. Purification by column chromatography $\left(\mathrm{SiO}_{2}\right.$, petroleum ether) yielded enyne s13 (606 mg, 4.26 $\mathrm{mmol}, 85 \%)$ as a colorless oil.

${ }^{1} \mathrm{H}$ NMR (400 MHz, $\left.\mathrm{CDCl}_{3}\right), \delta(\mathrm{ppm}):$ 7.48-7.45 (m, $\left.2 \mathrm{H}\right), 7.34-7.31(\mathrm{~m}, 3 \mathrm{H}), 5.42(\mathrm{~s}, 1 \mathrm{H}), 5.32(\mathrm{~s}, 1$ $\mathrm{H}), 2.01$ (s, $3 \mathrm{H}) ;{ }^{13} \mathrm{C} \mathrm{NMR}\left(100 \mathrm{MHz}, \mathrm{CDCl}_{3}\right) \delta$ (ppm): 131.6 (2), 128.3, 128.2 (2), 126.9, 123.3, 122.0, 90.6, 88.4, 23.5; IR (film) $v=3060$ (w), 2975 (w), 2953 (w), 2922 (w), 1802 (w), 1675 (w), 1611 (m), $1488(\mathrm{~m}), 1441(\mathrm{~m}), 1372(\mathrm{w}), 1313(\mathrm{~m}), 1198(\mathrm{w}), 1157(\mathrm{w}), 1069(\mathrm{w}), 1026(\mathrm{w}), 1011(\mathrm{w}), 894(\mathrm{~s})$, 752 (s), 688 (s), 560 (m), 536 (w), 510 (m), 485 (w), 456 (w); MS (El, 70 eV): m/z (\%): 142 (100), 127 (22), 115 (23), 101 (4), 89 (3), 77 (7), 63 (5), 51 (4); HRMS (El, $70 \mathrm{eV}$ ): calcd for [C $\mathrm{C}_{11} \mathrm{H}_{10}$ ]: 142.0783; found: $142.0782 ; \boldsymbol{R}_{\mathfrak{f}}=0.62$ (petroleum ether).

The analytical data were consistent with those published in the literature ${ }^{[15]}$.

\section{2-cyclopropyl-4-phenylbut-3-yn-2-ol s14 ${ }^{[17]}$}

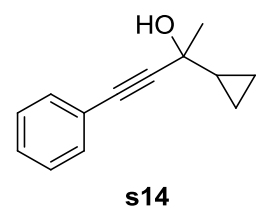

Phenylacetylene ( $550 \mu \mathrm{L}, 5 \mathrm{mmol}, 1$ eq.) was loaded in a dry flask under nitrogen and dissolved in dry THF (10 mL). The solution was cooled down to $-78^{\circ} \mathrm{C}$ and $n$-BuLi $(2.5 \mathrm{M}$ in hexanes, $2 \mathrm{~mL}, 5 \mathrm{mmol}, 1$ eq.) was added slowly. The resulting solution was brought to room temperature and stirred for 20 minutes. The reaction mixture was cooled down to $-78{ }^{\circ} \mathrm{C}$ again and acetylcyclopropane $(420 \mu \mathrm{L}, 5$ $\mathrm{mmol}, 1$ eq.) was added dropwise. The reaction mixture was then allowed to warm up to room temperature. After stirring for 40 minutes, it was quenched by addition of a saturated $\mathrm{NH}_{4} \mathrm{Cl}$ solution. The aqueous layer was extracted three times with EtOAc, the organic layers were combined, washed with brine, dried over anhydrous $\mathrm{Na}_{2} \mathrm{SO}_{4}$, filtered and concentrated under reduced pressure, yielding alcohol s14 (890 mg, $4.78 \mathrm{mmol}, 96 \%)$ as a colorless oil.

${ }^{1} \mathrm{H}$ NMR (400 MHz, CDCl $), \delta(\mathrm{ppm}): 7.39(\mathrm{~m}, 2 \mathrm{H}), 7.29(\mathrm{~m}, 3 \mathrm{H}), 2.10(\mathrm{~d}, J=10.4 \mathrm{~Hz}, 1 \mathrm{H}), 1.66(\mathrm{~s}, 3$ $\mathrm{H}), 1.23(\mathrm{~m}, 1 \mathrm{H}), 0.68(\mathrm{~m}, 1 \mathrm{H}), 0.60-0.47(\mathrm{~m}, 3 \mathrm{H}) ;{ }^{13} \mathbf{C ~ N M R}\left(100 \mathrm{MHz}, \mathrm{CDCl}_{3}\right) \delta(\mathrm{ppm}): 130.0(2)$, 126.6, 126.5 (2), 120.9, 88.2, 82.1, 68.8, 28.1, 20.4, 0.9, 0.0; IR (film) $v=3381$ (m), 3082 (w), 3006 (w), $2981(w), 2931(w), 1598(w), 1573(w), 1489(m), 1444(m), 1367(m), 1276(m), 1208(w), 1183$ 
(w), $1118(\mathrm{~m}), 1071(\mathrm{~m}), 1048(\mathrm{~m}), 1022(\mathrm{~m}), 951(\mathrm{~m}), 922(\mathrm{~m}), 869(\mathrm{w}), 826(\mathrm{w}), 800(\mathrm{w}), 755(\mathrm{~s}), 690$

(s), 642 (w), $575(\mathrm{~m}), 523$ (m), 482 (w); MS (El, $70 \mathrm{eV}): \mathrm{m} / \mathrm{z}(\%): 186$ (12), 171 (47), 158 (100), 152

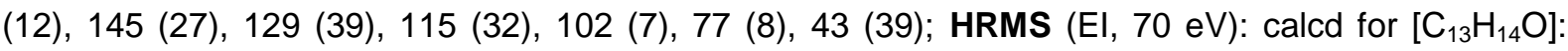
186.1045; found: $186.1044 ; \boldsymbol{R}_{\mathbf{f}}=0.22$ (petroleum ether/ethyl acetate 10/1).

\section{(3-cyclopropylbut-3-en-1-yn-1-yl)benzene s15 ${ }^{[17]}$}

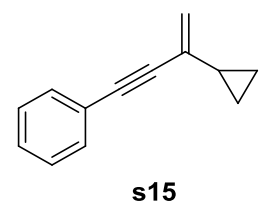

2-Cyclopropyl-4-phenylbut-3-yn-2-ol s14 $(800.0 \mathrm{mg}, 4.3 \mathrm{mmol}, 1$ eq.) was loaded in a dry flask under nitrogen and dissolved in dry DCM $(10 \mathrm{~mL})$. The solution was cooled down to $0{ }^{\circ} \mathrm{C}$ and $\mathrm{NEt}_{3}(3 \mathrm{~mL}$, $21.5 \mathrm{mmol}, 5$ eq.), then $\mathrm{MsCl}$ ( $832 \mu \mathrm{L}, 10.75 \mathrm{mmol}, 2.5$ eq.) were added. The reaction mixture was stirred at $0{ }^{\circ} \mathrm{C}$ for 15 minutes, then quenched by addition of a saturated $\mathrm{NH}_{4} \mathrm{Cl}$ solution. The aqueous layer was extracted thrice with EtOAc, the organic layers were combined, washed with brine, dried over anhydrous $\mathrm{Na}_{2} \mathrm{SO}_{4}$, filtered and concentrated under reduced pressure. Purification by column chromatography ( $\mathrm{SiO}_{2}$, petroleum ether) yielded enyne s15 (492 mg, $\left.2.92 \mathrm{mmol}, 68 \%\right)$ as a yellow oil. ${ }^{1} \mathrm{H}$ NMR $\left(400 \mathrm{MHz}, \mathrm{CDCl}_{3}\right), \delta(\mathrm{ppm}): 7.43(\mathrm{~m}, 2 \mathrm{H}), 7.31(\mathrm{~m}, 3 \mathrm{H}), 5.42(\mathrm{dd}, J=11.6,1.6 \mathrm{~Hz}, 2 \mathrm{H})$, $1.65(\mathrm{~m}, 1 \mathrm{H}), 0.82(\mathrm{~m}, 2 \mathrm{H}), 0.75(\mathrm{~m}, 2 \mathrm{H}) ;{ }^{13} \mathrm{C}$ NMR (100 MHz, $\left.\mathrm{CDCl}_{3}\right) \delta(\mathrm{ppm}): 133.9,131.6(2)$, 128.3, 128.2 (2), 123.1, 118.9, 89.5, 86.6, 16.5, 5.9 (2); IR (film) $v=3083$ (w), 3007 (w), 1780 (w), $1601(\mathrm{~m}), 1571(\mathrm{w}), 1489(\mathrm{~m}), 1442(\mathrm{w}), 1422(\mathrm{w}), 1386(\mathrm{w}), 1349(\mathrm{w}), 1303(\mathrm{w}), 1175(\mathrm{w}), 1096(\mathrm{w})$, $1064(\mathrm{w}), 1051(\mathrm{w}), 1024(\mathrm{w}), 959(\mathrm{~m}), 909(\mathrm{~m}), 879(\mathrm{~m}), 817(\mathrm{w}), 752(\mathrm{~s}), 688(\mathrm{~s}), 655(\mathrm{w}), 621(\mathrm{w})$, 587 (w), $522(\mathrm{~m}), 504$ (w), 446 (w); MS (El, $70 \mathrm{eV}): \mathrm{m} / z$ (\%): 168 (100), 152 (34), 139 (8), 127 (29), 115 (13), 102 (4), 89 (4), 77 (8); HRMS (El, $70 \mathrm{eV}$ ): calcd for [ $\left.\mathrm{C}_{13} \mathrm{H}_{12}\right]$ : 168.0939; found: 168.0938; $\boldsymbol{R}_{\mathbf{f}}=$ 0.70 (pentane). The analytical data were consistent with those published in the literature ${ }^{[17]}$.

\section{1-phenylpent-4-en-2-yn-1-ol s16 ${ }^{[18]}$}

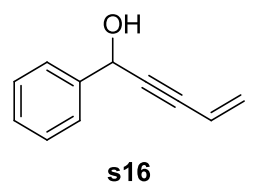

$\mathrm{Pd}\left(\mathrm{PPh}_{3}\right)_{4}(10.4 \mathrm{mg}, 0.009 \mathrm{mmol}, 0.3 \mathrm{~mol} \%)$ and Cul (11.4 mg, $\left.0.06 \mathrm{mmol}, 2 \mathrm{~mol} \%\right)$ were loaded in a dry schlenk under nitrogen. Dry $\mathrm{NEt}_{3}(1.5 \mathrm{~mL})$ was added and the mixture was cooled down to $0{ }^{\circ} \mathrm{C}$. 1 Phenyl-2-propyn-1-ol (396.5 mg, $3 \mathrm{mmol}, 1$ eq.) and vinyl bromide ( $423 \mu \mathrm{L}, 6 \mathrm{mmol}, 2$ eq.) were subsequently added and the mixture was warmed up to room temperature and stirred overnight. Water was added to the reaction mixture. The aqueous layer was extracted with $\mathrm{Et}_{2} \mathrm{O}$ :pentane (1:1) and the combined organic layers were washed with a $1 \mathrm{~N} \mathrm{HCl}$ solution, a saturated $\mathrm{NH}_{4} \mathrm{Cl}$ solution and brine, dried over anhydrous $\mathrm{Na}_{2} \mathrm{SO}_{4}$, filtered and concentrated under reduced pressure. Purification by column chromatography $\left(\mathrm{SiO}_{2}\right.$, petroleum ether/ethyl acetate $10 / 1$ to $\left.5 / 1\right)$ yielded enyne s16 (360 mg, $2.27 \mathrm{mmol}, 76 \%$ ) as a yellow oil. 
${ }^{1} \mathrm{H}$ NMR $\left(400 \mathrm{MHz}, \mathrm{CDCl}_{3}\right), \delta(\mathrm{ppm}): 7.55(\mathrm{~d}, J=7.3 \mathrm{~Hz}, 2 \mathrm{H}), 7.41-7.32(\mathrm{~m}, 3 \mathrm{H}), 5.88$ (ddd, $J=17.6$, 11.1, $1.7 \mathrm{~Hz}, 1 \mathrm{H}$ ), 5.70 (dd, $J=17.6,2.0 \mathrm{~Hz}, 1 \mathrm{H}$ ), 5.59 (d, $J=5.5 \mathrm{~Hz}, 1 \mathrm{H}$ ), 5.53 (dd, $J=11.1,2.0$ $\mathrm{Hz}, 1 \mathrm{H}), 2.21(\mathrm{~d}, J=6.0 \mathrm{~Hz}, 1 \mathrm{H}) ;{ }^{13} \mathrm{C}$ NMR $\left(100 \mathrm{MHz}, \mathrm{CDCl}_{3}\right) \delta(\mathrm{ppm}): 140.6,128.7$ (2), 128.4, 127.8, 126.7 (2), 116.5, 89.3, 85.3, 65.0; IR (film) $v=3352$ (m), 3031 (w), 2878 (w), 1601 (m), 1493 $(\mathrm{m}), 1453(\mathrm{~m}), 1411(\mathrm{~m}), 1276(\mathrm{~m}), 1192(\mathrm{~m}), 1152(\mathrm{~s}), 1077(\mathrm{w}), 1039(\mathrm{~m}), 974(\mathrm{~s}), 920(\mathrm{~m}), 831(\mathrm{w})$, 798 (w), 756 (m), 726 (m), 697 (s), 634 (m), 545 (w); MS (El, $70 \mathrm{eV}):$ m/z (\%): 157 (36), 139 (9), 129 (100), 115 (28), 105 (6), 89 (3), 77 (16), 63 (4), 51 (10); HRMS (El, 70 eV): calcd for [C ${ }_{11} \mathrm{H}_{10} \mathrm{O}$ ]: 158.0732; found: $158.0720 ; \boldsymbol{R}_{\mathfrak{f}}=0.53$ (petroleum ether/ethyl acetate 4/1).

The analytical data were consistent with those published in the literature ${ }^{[18]}$.

\section{1-phenylpent-4-en-2-yn-1-yl acetate $s 17^{[19]}$}

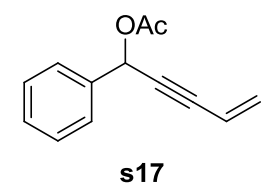

To a solution of 1-phenylpent-4-en-2-yn-1-ol s16 (316.0 mg, $2 \mathrm{mmol}$, 1eq.) in DCM (5 mL) were added DMAP (24.4 mg, $0.2 \mathrm{mmol}, 0.1$ eq.) and $\mathrm{NEt}_{3}(418 \mu \mathrm{L}, 3 \mathrm{mmol}, 1.5$ eq.). The resulting mixture was cooled down to $0{ }^{\circ} \mathrm{C}$ and stirred for 15 minutes. $\mathrm{Ac}_{2} \mathrm{O}(176 \mu \mathrm{L}, 2.4 \mathrm{mmol}, 1.2 \mathrm{eq}$.) was added and the reaction mixture was slowly warmed up to room temperature and stirred for 4 hours. Water was added to the reaction mixture. The aqueous layer was extracted with DCM and the combined organic layers were dried over anhydrous $\mathrm{Na}_{2} \mathrm{SO}_{4}$, filtered and concentrated under reduced pressure. Purification by column chromatography $\left(\mathrm{SiO}_{2}\right.$, petroleum ether/ethyl acetate 10/1) yielded enyne s17 (371 mg, 1.85 $\mathrm{mmol}, 93 \%)$ as a colorless oil.

${ }^{1}$ H NMR (400 MHz, $\left.\mathrm{CDCl}_{3}\right), \delta(\mathrm{ppm}): 7.53(\mathrm{~m}, 2 \mathrm{H}), 7.41-7.34(\mathrm{~m}, 3 \mathrm{H}), 6.58(\mathrm{~s}, 1 \mathrm{H}), 5.86$ (ddd, $J=$ 17.6, 11.1, 1.6 Hz, $1 \mathrm{H}$ ), 5.72 (dd, $J=17.6,2.1 \mathrm{~Hz}, 1 \mathrm{H}$ ), 5.55 (dd, $J=11.1,2.1 \mathrm{~Hz}, 1 \mathrm{H}$ ), 2.11 (s, $3 \mathrm{H}$ ); ${ }^{13} \mathrm{C}$ NMR $\left(100 \mathrm{MHz}, \mathrm{CDCl}_{3}\right) \delta$ (ppm): 169.8, 137.1, 128.9, 128.7 (2), 128.4, 127.7 (2), 116.3, 86.1, 85.7, 66.0, 21.1; IR (film) $v=3035$ (w), 1737 (s), 1600 (w), 1495 (w), 1455 (w), 1413 (w), 1369 (m), $1220(\mathrm{~s}), 1159(\mathrm{~m}), 1077(\mathrm{w}), 1014(\mathrm{~m}), 952(\mathrm{~m}), 930(\mathrm{~m}), 902(\mathrm{~m}), 840(\mathrm{w}), 803(\mathrm{w}), 753(\mathrm{~m}), 697(\mathrm{~s})$, $629(\mathrm{w}), 603(\mathrm{w}), 546(\mathrm{~m}), 474(\mathrm{w})$; HRMS (El, $70 \mathrm{eV})$ : calcd for $\left[\mathrm{C}_{13} \mathrm{H}_{12} \mathrm{O}_{2}\right]$ : 200.0837; found: 200.0837; $\boldsymbol{R}_{\mathfrak{f}}=0.68$ (petroleum ether/ethyl acetate 4/1).

The analytical data were consistent with those published in the literature ${ }^{[20]}$.

\section{1-phenylpent-4-en-2-yn-1-one $s 18^{[18]}$}

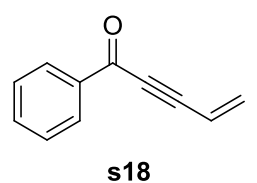

1-Phenylpent-4-en-2-yn-1-ol s16 (316.6 mg, 2 mmol, 1 eq.) was dissolved in dry DCM (40 mL) at $0{ }^{\circ} \mathrm{C}$ and Dess-Martin periodinane ( $2.12 \mathrm{~g}, 5 \mathrm{mmol}, 2.5 \mathrm{eq}$.) was added. The reaction mixture was warmed up to room temperature and stirred for 1.5 hours. The solvent was removed under reduced pressure and a saturated $\mathrm{NaHCO}_{3}$ solution was added. The aqueous layer was extracted three times with $\mathrm{Et}_{2} \mathrm{O}$ 
and the combined organic layers were dried over anhydrous $\mathrm{Na}_{2} \mathrm{SO}_{4}$, filtered and concentrated under reduced pressure. Purification by column chromatography $\left(\mathrm{SiO}_{2}\right.$, pentane/ $\left.\mathrm{Et}_{2} \mathrm{O} 4 / 1\right)$ yielded enyne s18 (166 mg, $1.06 \mathrm{mmol}, 53 \%$ ) as a yellow oil.

${ }^{1} \mathrm{H}$ NMR $\left(400 \mathrm{MHz}, \mathrm{CDCl}_{3}\right), \delta(\mathrm{ppm}): 8.14$ (d, $\left.J=7.3 \mathrm{~Hz}, 2 \mathrm{H}\right), 7.61$ (t, $\left.J=7.4 \mathrm{~Hz}, 1 \mathrm{H}\right), 7.49$ (t, $J=7.7$ $\mathrm{Hz}, 2 \mathrm{H}), 6.06$ (d, J = 7.2 Hz, $2 \mathrm{H}$ ), 5.87 (dd, $J=7.2,1.2 \mathrm{~Hz}, 1 \mathrm{H}) ;{ }^{13} \mathrm{C} \mathbf{N M R}\left(100 \mathrm{MHz}, \mathrm{CDCl}_{3}\right) \delta$ (ppm): 177.9, 136.7, 134.1, 133.1, 129.6 (2), 128.6 (2), 115.3, 91.2, 87.1; IR (film) $v=3268$ (w), 3064 (w), 2195 (s) $1638(\mathrm{~s}), 1597(\mathrm{~m}), 1580(\mathrm{~m}), 1450(\mathrm{~m}), 1412(\mathrm{w}), 1313(\mathrm{w}), 1261(\mathrm{~s}), 1176(\mathrm{~m}), 1137$ (w), $1071(\mathrm{w}), 1040(\mathrm{~m}), 1022(\mathrm{w}), 968(\mathrm{w}), 939(\mathrm{~m}), 886(\mathrm{~m}), 794(\mathrm{w}), 697(\mathrm{~s}), 619(\mathrm{w}) ; 556(\mathrm{w})$; HRMS (El, $70 \mathrm{eV}$ ): calcd for $\left[\mathrm{C}_{11} \mathrm{H}_{8} \mathrm{O}\right]$ : 156.0575; found: 156.0574; $\boldsymbol{R}_{\mathrm{f}}=0.69$ (petroleum ether/ethyl acetate 4/1).

The analytical data were consistent with those published in the literature ${ }^{[18]}$.

\section{dec-1-en-3-yne s19}

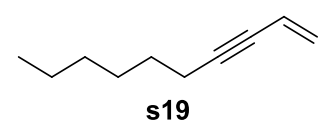

According to GP-2, $\mathrm{PdCl}_{2}\left(\mathrm{PPh}_{3}\right)_{2}(70.2 \mathrm{mg}, 0.1 \mathrm{mmol}, 4 \mathrm{~mol} \%)$, Cul (28.6 mg, $\left.0.15 \mathrm{mmol}, 6 \mathrm{~mol} \%\right)$, $\mathrm{PPh}_{3}$ (26.2 mg, $0.1 \mathrm{mmol}, 4$ mol\%), dry NEt 3 (3.5 mL, $25 \mathrm{mmol}, 10$ eq.), 1-octyne ( $369 \mu \mathrm{L}, 2.5 \mathrm{mmol}, 1$ eq.), and vinylbromide ( $1 \mathrm{M}$ in THF, $5 \mathrm{~mL}, 5 \mathrm{mmol}, 2$ eq.) were stirred in dry THF (10 mL) under nitrogen at $50{ }^{\circ} \mathrm{C}$ for 2 hours. Purification by column chromatography $\left(\mathrm{SiO}_{2}\right.$, pentane) yielded enyne s19 (335 mg, $2.46 \mathrm{mmol}, 98 \%)$ as a yellow oil.

${ }^{1} \mathrm{H}$ NMR (400 MHz, $\mathrm{CDCl}_{3}$ ), $\delta(\mathrm{ppm}): 5.77$ (ddt, $J=17.5,11.0,2.1 \mathrm{~Hz}, 1 \mathrm{H}$ ), 5.53 (dd, $J=17.5,2.2 \mathrm{~Hz}$, $1 \mathrm{H}), 5.36$ (dd, $J=11.0,2.2 \mathrm{~Hz}, 1 \mathrm{H}$ ), 2.29 (dt, $J=7.1,2.0 \mathrm{~Hz}, 2 \mathrm{H}), 1.56-1.49(\mathrm{~m}, 2 \mathrm{H}), 1.43-1.26(\mathrm{~m}$, $6 \mathrm{H}), 0.90-0.86(\mathrm{~m}, 3 \mathrm{H}) ;{ }^{13} \mathrm{C}$ NMR $\left(100 \mathrm{MHz}, \mathrm{CDCl}_{3}\right) \delta(\mathrm{ppm}): 125.3,117.7,91.2,79.3,31.3,28.7$, 28.6, 22.5, 19.3, 14.0; IR (film) $v=2955$ (s), 2929 (vs), 2858 (m), 1677 (w), 1607 (w), 1465 (m), 1379 (w), 1326 (w), 1255 (w), 973 (w), $913(\mathrm{~m}), 725$ (w); MS (El, $70 \mathrm{eV}): \mathrm{m} / \mathrm{z}(\%): 136$ (18), 121 (20), 107 (63), 93 (44), 79 (100), 67 (25), 55 (12), 41 (26); HRMS (El, $70 \mathrm{eV}$ ): calcd for $\left[\mathrm{C}_{10} \mathrm{H}_{16}\right]$ : 136.1252; found: $136.1251 ; \boldsymbol{R}_{\mathrm{f}}=0.78$ (petroleum ether).

The analytical data were consistent with those published in the literature ${ }^{[21]}$.

\section{tert-butyl(pent-4-en-2-yn-1-yloxy)diphenylsilane s20 ${ }^{[22]}$}

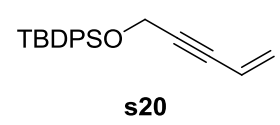

$\mathrm{Pd}\left(\mathrm{PPh}_{3}\right)_{4}(5.2 \mathrm{mg}, 0.0045 \mathrm{mmol}, 0.2 \mathrm{~mol} \%)$ and $\mathrm{Cul}(6.0 \mathrm{mg}, 0.03 \mathrm{mmol}, 1.4 \mathrm{~mol} \%)$ were loaded in a dry schlenk under nitrogen and dissolved in diethylamine $(1.25 \mathrm{~mL})$. Dry THF $(5 \mathrm{~mL})$ was added and the mixture was cooled down to $0{ }^{\circ} \mathrm{C}$. tert-Butyldiphenyl(prop-2-yn-1-yloxy)silane (662.6 mg, 2.25 mmol, 1 eq.) and vinyl bromide ( $317 \mu \mathrm{L}, 4.5 \mathrm{mmol}, 2$ eq.) were subsequently added and the mixture was gradually warmed up to room temperature and stirred overnight. The reaction mixture was poured into iced water. The aqueous layer was extracted three times with $\mathrm{Et}_{2} \mathrm{O}$ and the combined organic layers were washed with a saturated $\mathrm{NH}_{4} \mathrm{Cl}$ solution, water, then brine and dried over anhydrous $\mathrm{Na}_{2} \mathrm{SO}_{4}$, filtered and concentrated under reduced pressure. Purification by column chromatography 
$\left(\mathrm{SiO}_{2}\right.$, petroleum ether/ethyl acetate $90 / 1$ to $\left.40 / 1\right)$ yielded enyne s20 (655 mg, $\left.2.04 \mathrm{mmol}, 91 \%\right)$ as a colorless oil.

${ }^{1} \mathrm{H}$ NMR (400 MHz, $\left.\mathrm{CDCl}_{3}\right), \delta(\mathrm{ppm}): 7.72(\mathrm{~m}, 4 \mathrm{H}), 7.45-7.37(\mathrm{~m}, 6 \mathrm{H}), 5.77(\mathrm{dd}, J=17.6,11.1 \mathrm{~Hz}, 1$ H), 5.56 (dd, $J=17.6,1.8 \mathrm{~Hz}, 1 \mathrm{H}), 5.44$ (dd, $J=11.1,1.9 \mathrm{~Hz}, 1 \mathrm{H}), 4.44(\mathrm{~s}, 2 \mathrm{H}), 1.07$ (s, $9 \mathrm{H}) ;{ }^{13} \mathrm{C}$ NMR $\left(100 \mathrm{MHz}, \mathrm{CDCl}_{3}\right) \delta$ (ppm): 135.7, 133.2, 129.8, 127.7, 126.9, 116.9, 88.2, 83.8, 53.1, 26.7, 19.2; IR (film) $v=3071$ (w), 3049 (w), 3013 (w), 2959 (m), 2931 (m), 2894 (m), 2858 (m), 1609 (w), $1590(\mathrm{w}), 1472(\mathrm{~m}), 1428(\mathrm{~m}), 1391(\mathrm{w}), 1372(\mathrm{~m}), 1260(\mathrm{w}), 1163(\mathrm{~m}), 1111(\mathrm{~s}), 1077(\mathrm{~s}), 1026(\mathrm{~m})$, $997(\mathrm{~m}), 972(\mathrm{~m}), 922(\mathrm{w}), 823(\mathrm{~m}), 738(\mathrm{~m}), 701(\mathrm{~s}), 612(\mathrm{~m}), 505(\mathrm{~s}), 490(\mathrm{~s}), 416(\mathrm{w}) ;$ MS (El, 70 eV): m/z (\%): 320 (5), 305 (4), 263 (88), 233 (100), 211 (3), 199 (4), 181 (3), 105 (2); HRMS (El, 70 $\mathrm{eV}$ ): calcd for $\left[\mathrm{C}_{21} \mathrm{H}_{24} \mathrm{OSi}\right]$ : 320.1596; found: $320.1597 ; \boldsymbol{R}_{\mathfrak{f}}=0.50$ (petroleum ether/ethyl acetate $40 / 1$ ).

\section{4-methyl- $N$-(pent-4-en-2-yn-1-yl)benzenesulfonamide s21 ${ }^{[22]}$}

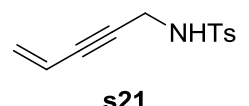

$\mathrm{Pd}\left(\mathrm{PPh}_{3}\right)_{4}(11.6 \mathrm{mg}, 0.01 \mathrm{mmol}, 0.2 \mathrm{~mol} \%)$ and $\mathrm{Cul}(13.3 \mathrm{mg}, 0.07 \mathrm{mmol}, 1.4 \mathrm{~mol} \%)$ were loaded in a dry schlenk under nitrogen and dissolved in diethylamine $(2.5 \mathrm{~mL})$. Dry THF $(6 \mathrm{~mL})$ was added and the mixture was cooled down to $0{ }^{\circ} \mathrm{C}$. 4-methyl- $N$-(prop-2-yn-1-yl)benzenesulfonamide $(1.046 \mathrm{~g}, 5 \mathrm{mmol}$, 1 eq.) and vinyl bromide ( $1 \mathrm{M}$ in THF, $10 \mathrm{~mL}, 10 \mathrm{mmol}, 2$ eq.) were subsequently added and the mixture was gradually warmed up to room temperature and stirred overnight. The reaction mixture was poured into iced water. The aqueous layer was extracted three times with $\mathrm{Et}_{2} \mathrm{O}$ and the combined organic layers were washed with a saturated $\mathrm{NH}_{4} \mathrm{Cl}$ solution, water, then brine and dried over anhydrous $\mathrm{Na}_{2} \mathrm{SO}_{4}$, filtered and concentrated under reduced pressure. Purification by column chromatography $\left(\mathrm{SiO}_{2}\right.$, petroleum ether/ethyl acetate 5/1) yielded enyne s21 (968 mg, $4.11 \mathrm{mmol}, 82$ $\%)$ as a beige solid.

${ }^{1} \mathrm{H}$ NMR $\left(400 \mathrm{MHz}, \mathrm{CDCl}_{3}\right), \delta(\mathrm{ppm}): 7.78(\mathrm{~d}, J=8.1 \mathrm{~Hz}, 2 \mathrm{H}), 7.31(\mathrm{~d}, J=8.1 \mathrm{~Hz}, 2 \mathrm{H}), 5.58(\mathrm{dd}, J=$ 17.9, $10.7 \mathrm{~Hz}, 1 \mathrm{H}$ ), 5.43-5.39 (m, $2 \mathrm{H}), 4.51$ (br s, $1 \mathrm{H}), 3.96$ (d, J = 5.9 Hz, $2 \mathrm{H}$ ), $2.42(\mathrm{~s}, 3 \mathrm{H}) ;{ }^{13} \mathbf{C}$ NMR $\left(100 \mathrm{MHz}, \mathrm{CDCl}_{3}\right) \delta$ (ppm): 143.7, 136.7, 129.7 (2), 127.7, 127.5 (2), 116.1, 83.8, 83.4, 33.7, 21.5; IR (film) $v$ = 3266 (s), 3032 (w), 2976 (w), 2923 (w), 2859 (w), 1597 (w), 1495 (w), $1434(\mathrm{~m})$, $1322(\mathrm{~s}), 1305$ (m), 1289 (w), 1238 (w), 1185 (w), $1154(\mathrm{~s}), 1091$ (s), 1067 (m), 1019 (w), 973 (w), 938 (m), $910(\mathrm{~m}), 841(\mathrm{~m}), 813(\mathrm{~s}), 559(\mathrm{~m}), 538(\mathrm{~m}), 516(\mathrm{~m}), 417(\mathrm{w}) ;$ MS (ESI): m/z (\%): 258 (100); HRMS (ESI): calcd for $\left[\mathrm{C}_{12} \mathrm{H}_{13} \mathrm{NO}_{2} \mathrm{SNa}^{+}\right]$: 258.0559; found: 258.0549; $\boldsymbol{R}_{\mathbf{f}}=0.33$ (petroleum ether/ethyl acetate $4 / 1$ ); Melting point: $86^{\circ} \mathrm{C}$.

The analytical data were consistent with those published in the literature ${ }^{[22]}$.

\section{$\mathrm{N}$-allyl-4-methyl- $\mathrm{N}$-(pent-4-en-2-yn-1-yl)benzenesulfonamide s22}

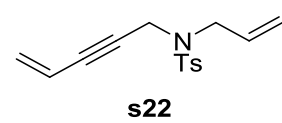

4-methyl- $N$-(pent-4-en-2-yn-1-yl)benzenesulfonamide s21 (953 mg, $4.05 \mathrm{mmol}, 1$ eq.) and potassium carbonate (2.24 g, $16.2 \mathrm{mmol}, 4$ eq.) were suspended in acetonitrile $(40 \mathrm{~mL})$. Allyl bromide $(700 \mu \mathrm{L}$, $8.1 \mathrm{mmol}, 2$ eq.) was added and the resulting mixture was stirred overnight at room temperature. The 
potassium carbonate was filtered off and the filtrate was concentrated under reduced pressure, yielding enyne s22 (1.10 g, $3.99 \mathrm{mmol}, 99 \%)$ as a light brown solid.

${ }^{1} \mathrm{H}$ NMR $\left(400 \mathrm{MHz}, \mathrm{CDCl}_{3}\right), \delta(\mathrm{ppm}): 7.74(\mathrm{~d}, J=8.3, \mathrm{~Hz}, 2 \mathrm{H}), 7.28(\mathrm{~d}, J=8.0 \mathrm{~Hz}, 2 \mathrm{H}), 5.75(\mathrm{~m}, 1 \mathrm{H})$, $5.51(\mathrm{dt}, J=17.2,11.3,1.9 \mathrm{~Hz}, 1 \mathrm{H}), 5.38-5.22(\mathrm{~m}, 4 \mathrm{H}), 4.19(\mathrm{~d}, J=1.6 \mathrm{~Hz}, 2 \mathrm{H}), 3.81(\mathrm{~d}, J=6.4 \mathrm{~Hz}$, $2 \mathrm{H}), 2.41$ (s, $3 \mathrm{H}) ;{ }^{13} \mathrm{C}$ NMR (100 MHz, $\left.\mathrm{CDCl}_{3}\right) \delta(\mathrm{ppm}): 143.4,136.0,132.1,129.4(2), 127.8(2)$, 127.2, 119.8, 116.3, 84.2, 82.5, 49.2, 36.6, 21.5; IR (film) $v=2980$ (w), 2919 (w), $1644(w), 1598(\mathrm{~m})$, $1495(w), 1418(w), 1348(s), 1306(w), 1290(w), 1257(w), 1161(s), 1119(w), 1092(m), 1059(w)$, $1018(\mathrm{w}), 991(\mathrm{w}), 974(\mathrm{w}), 930(\mathrm{~m}), 898(\mathrm{~m}), 815(\mathrm{~m}), 751(\mathrm{~m}), 706(\mathrm{~s}), 663(\mathrm{~m}), 576(\mathrm{~m}), 546(\mathrm{~m})$; MS (ESI): $\mathrm{m} / z$ (\%): 298 (100), 276 (40); HRMS (ESI): calcd for $\left[\mathrm{C}_{15} \mathrm{H}_{17} \mathrm{NO}_{2} \mathrm{~S}^{+}\right]$: 276.1053; found: 276.1050; $\boldsymbol{R}_{\mathrm{f}}=0.56$ (petroleum ether/ethyl acetate 5/1); Melting point: $31^{\circ} \mathrm{C}$.

The analytical data were consistent with those published in the literature ${ }^{[23]}$.

\section{2,2-dimethyl-7-phenylhept-4-yn-3-one $s 23^{[24]}$}

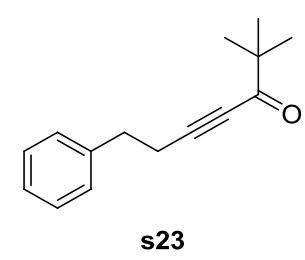

Dry $\mathrm{NEt}_{3}(20 \mathrm{~mL})$ was loaded in a dry flask under nitrogen. 4-Phenyl-1-butyne $(1.41 \mathrm{~mL}, 10 \mathrm{mmol}, 1$ eq.), pivaloyl chloride (1.23 mL, $10 \mathrm{mmol}, 1$ eq.), $\mathrm{PdCl}_{2}\left(\mathrm{PPh}_{3}\right)_{2}(17.5 \mathrm{mg}, 0.025 \mathrm{mmol}, 0.25 \mathrm{~mol} \%$ ) and Cul (23.8 mg, $0.125 \mathrm{mmol}, 1.25 \mathrm{~mol} \%$ ) were subsequently added and the resulting mixture was stirred at $50{ }^{\circ} \mathrm{C}$ (oil bath) for 20 hours. $\mathrm{MeOH}(5 \mathrm{~mL})$ was added and the solvent was removed under reduced pressure. The resulting residue was dissolved in $\mathrm{Et}_{2} \mathrm{O}(100 \mathrm{~mL})$ and the precipitate was filtered off. The filtrate was washed with a $10 \% \mathrm{HCl}$ solution, a saturated $\mathrm{NH}_{4} \mathrm{Cl}$ solution, water and brine, then dried over anhydrous $\mathrm{Na}_{2} \mathrm{SO}_{4}$, filtered and concentrated under reduced pressure. Purification by column chromatography $\left(\mathrm{SiO}_{2}\right.$, petroleum ether/ethyl acetate 20/1 to 10/1) yielded ketone s23 $(2.01 \mathrm{~g}$, $9.36 \mathrm{mmol}, 94 \%)$ as a yellow oil.

${ }^{1} \mathrm{H}$ NMR (400 MHz, $\left.\mathrm{CDCl}_{3}\right), \delta(\mathrm{ppm}): 7.32-7.29(\mathrm{~m}, 2 \mathrm{H}), 7.26-7.21(\mathrm{~m}, 3 \mathrm{H}), 2.91(\mathrm{t}, J=7.4 \mathrm{~Hz}, 2 \mathrm{H})$, $2.70(\mathrm{t}, J=7.4 \mathrm{~Hz}, 2 \mathrm{H}), 1.13(\mathrm{~s}, 9 \mathrm{H}) ;{ }^{13} \mathrm{C}$ NMR $\left(100 \mathrm{MHz}, \mathrm{CDCl}_{3}\right) \delta(\mathrm{ppm}): 194.3,139.7,128.5(2)$, 128.4 (2), 126.6, 94.3, 79.4, 44.6, 34.0, 26.0 (3), 21.1; IR (film) $v=3064(\mathrm{w}), 3029(\mathrm{w}), 2970(\mathrm{~m}), 2931$ (w), $2869(w), 2211(\mathrm{~m}), 1666(\mathrm{~s}), 1496(\mathrm{w}), 1477(\mathrm{~m}), 1455(\mathrm{~m}), 1393(\mathrm{w}), 1364(\mathrm{w}), 1339(\mathrm{~m}), 1275$ $(\mathrm{m}), 1148(\mathrm{~s}), 1078(\mathrm{w}), 1031(\mathrm{w}), 1001(\mathrm{w}), 966(\mathrm{~m}), 944(\mathrm{~m}), 904(\mathrm{w}), 865(\mathrm{w}), 806(\mathrm{w}), 746(\mathrm{~m}), 699$ (m), $571(\mathrm{~m}), 484(\mathrm{w}), 426(\mathrm{w})$; HRMS (El, $70 \mathrm{eV})$ : calcd for $\left[\mathrm{C}_{15} \mathrm{H}_{18} \mathrm{O}\right.$ ]: 214.1358; found: 214.1356; $\boldsymbol{R}_{\mathbf{f}}$ $=0.36$ (petroleum ether/ethyl acetate 20/1).

(6,6-dimethyl-5-methylenehept-3-yn-1-yl)benzene s24 ${ }^{[25]}$

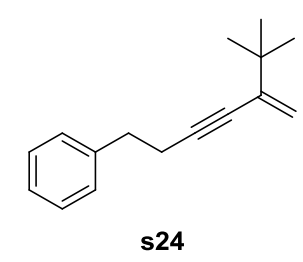


$\mathrm{PPh}_{3} \mathrm{CH}_{3} \mathrm{Br}$ (3.674 g, $10.29 \mathrm{mmol}, 1.1$ eq.) was suspended in dry THF (100 mL) and cooled down to $0^{\circ} \mathrm{C}$. $n$-BuLi (2.5 M in hexanes, $4.12 \mathrm{~mL}, 10.29 \mathrm{mmol}, 1.1$ eq.) was added dropwise under nitrogen and the resulting suspension was stirred for a further 30 minutes at this temperature, then cooled down to $78{ }^{\circ} \mathrm{C}$. 2,2-Dimethyl-7-phenylhept-4-yn-3-one s23 (2.00 g, $9.35 \mathrm{mmol}, 1$ eq.) in dry THF (20 mL) was added dropwise and the reaction mixture was stirred at this temperature for 6.5 hours. A saturated $\mathrm{NH}_{4} \mathrm{Cl}$ solution was added to quench the reaction, the aqueous layer was extracted with $\mathrm{Et}_{2} \mathrm{O}$ and the combined organic layers were washed with brine, dried over anhydrous $\mathrm{Na}_{2} \mathrm{SO}_{4}$, filtered and concentrated under reduced pressure. Purification by column chromatography $\left(\mathrm{SiO}_{2}\right.$, petroleum ether to petroleum ether/ethyl acetate 20/1) yielded enyne s24 (1.88 g, $8.83 \mathrm{mmol}, 94 \%)$ as a pale yellow oil.

${ }^{1} \mathrm{H}$ NMR (300 MHz, $\left.\mathrm{CDCl}_{3}\right), \delta(\mathrm{ppm}): 7.35-7.21(\mathrm{~m}, 5 \mathrm{H}), 5.21(\mathrm{dd}, J=10.3,1.5 \mathrm{~Hz}, 2 \mathrm{H}), 2.89$ (t, $J=$ $7.5 \mathrm{~Hz}, 2 \mathrm{H}$ ), 2.66 (t, $J=7.5 \mathrm{~Hz}, 2 \mathrm{H}), 1.11$ (s, $9 \mathrm{H}) ;{ }^{13} \mathrm{C} \mathrm{NMR}\left(75 \mathrm{MHz}, \mathrm{CDCl}_{3}\right) \delta$ (ppm): 142.0, 140.8, 128.5 (2), 128.3 (2), 126.2, 116.5, 89.8, 81.0, 35.9, 35.2, 28.9 (3), 21.5; IR (film) $v=3063$ (w), 3028 (w), $2955(\mathrm{~m}), 2928(\mathrm{~m}), 2866(\mathrm{~m}), 2211(\mathrm{~m}), 1601(\mathrm{~m}), 1496(\mathrm{~m}), 1479(\mathrm{~m}), 1454(\mathrm{~m}), 1383(\mathrm{w}), 1360$ $(\mathrm{m}), 1340(\mathrm{w}), 1192(\mathrm{~m}), 1077(\mathrm{w}), 1030(\mathrm{w}), 895(\mathrm{~s}), 744(\mathrm{~m}), 696(\mathrm{~s}), 651(\mathrm{~m}), 576(\mathrm{w}), 553(\mathrm{w}), 505$ $(\mathrm{m}), 477(\mathrm{w})$; HRMS (El, $70 \mathrm{eV}$ ): calcd for $\left[\mathrm{C}_{16} \mathrm{H}_{20}\right]$ : 212.1565; found: $212.1565 ; \boldsymbol{R}_{\mathbf{f}}=0.47$ (petroleum ether/ethyl acetate 90/1). 


\section{Iron-Catalyzed Cyclopropanation of Enynes.}

\section{General procedure 3 (GP-3): TBAFe-catalyzed cyclopropanation of enynes under thermal conditions.}

TBAFe $(5.2 \mathrm{mg}, 0.0125 \mathrm{mmol}, 2.5 \mathrm{~mol} \%)$ was loaded in a dry schlenk tube under nitrogen. The corresponding enyne ( $0.5 \mathrm{mmol}, 1 \mathrm{eq}$.), a solution of 4-nitroanisole $(0.38 \mathrm{mg}, 0.0025 \mathrm{mmol}, 0.5 \mathrm{~mol} \%)$ in dry 1,2-DCE $(250 \mu \mathrm{L})$ and ethyl diazoacetate $(63 \mu \mathrm{L}, 0.52 \mathrm{mmol}, 1.05$ eq.) were subsequently added and the reaction mixture was stirred at $40^{\circ} \mathrm{C}$ (heating block) for 24 hours. The reaction mixture was filtered through a plug of silica and eluted with $\mathrm{Et}_{2} \mathrm{O}$ or $\mathrm{EtOAc}$. The filtrate was concentrated under reduced pressure and the crude product was purified by column chromatography.

\section{General procedure 4 (GP-4): TBAFe-catalyzed cyclopropanation of enynes under photochemical conditions.}

TBAFe (10.3 mg, $0.025 \mathrm{mmol}, 5 \mathrm{~mol} \%)$ was loaded in a dry schlenk tube under nitrogen. The corresponding enyne $(0.5 \mathrm{mmol}, 1$ eq.), dry 1,4-dioxane $(125 \mu \mathrm{L})$, nitromethane $(25 \mu \mathrm{L})$, and ethyl diazoacetate ( $63 \mu \mathrm{L}, 0.52 \mathrm{mmol}, 1.05$ eq.) were subsequently added and the reaction mixture was stirred at room temperature under irradiation (75W Xe-lamp) for 20 hours. The reaction mixture was filtered through a plug of silica and eluted with $\mathrm{Et}_{2} \mathrm{O}$ or EtOAc. The filtrate was concentrated under reduced pressure and the crude product was purified by column chromatography.

ethyl 2-(4-phenylbut-1-yn-1-yl)cyclopropane-1-carboxylate 3

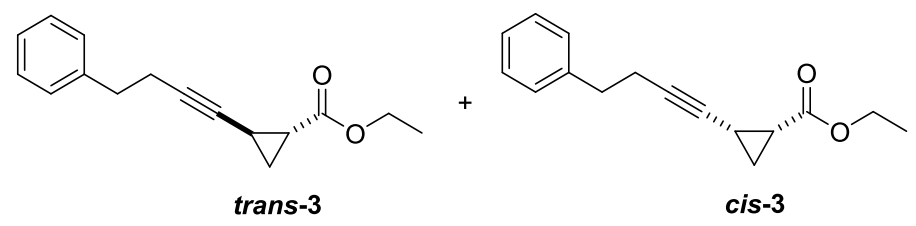

Thermal conditions:

According to GP-3, TBAFe (5.2 mg, $0.0125 \mathrm{mmol}, 2.5 \mathrm{~mol} \%$ ), 4-nitroanisole ( $0.38 \mathrm{mg}, 0.0025 \mathrm{mmol}$, $0.5 \mathrm{~mol} \%$ ) in 1,2-DCE (250 $\mu \mathrm{L})$, hex-5-en-3-yn-1-ylbenzene 1 (78.1 mg, $0.5 \mathrm{mmol}, 1$ eq.), and ethyl diazoacetate ( $63 \mu \mathrm{L}, 0.52 \mathrm{mmol}, 1.05$ eq.) were stirred at $40^{\circ} \mathrm{C}$ for 24 hours. The reaction mixture was filtered through a plug of silica and eluted with $\mathrm{Et}_{2} \mathrm{O}$. Purification by column chromatography $\left(\mathrm{SiO}_{2}\right.$, petroleum ether/ethyl acetate $40 / 1$ to $10 / 1$ ) yielded cyclopropane $3(86 \mathrm{mg}, 0.36 \mathrm{mmol}, 71 \%$ ) as a mixture of diastereomers, trans:cis-2.8:1.

\section{Photochemical conditions:}

According to GP-4, TBAFe (10.3 mg, $0.025 \mathrm{mmol}, 5 \mathrm{~mol} \%)$, 1,4-dioxane (125 $\mu \mathrm{L})$, nitromethane (25 $\mu \mathrm{L}$ ), hex-5-en-3-yn-1-ylbenzene 1 (78.1 mg, $0.5 \mathrm{mmol}, 1$ eq.), and ethyl diazoacetate (63 $\mu \mathrm{L}, 0.52$ $\mathrm{mmol}, 1.05$ eq.) were stirred at room temperature under irradiation for 20 hours. The reaction mixture was filtered through a plug of silica and eluted with $\mathrm{Et}_{2} \mathrm{O}$. The filtrate was concentrated under reduced pressure. Purification by column chromatography $\left(\mathrm{SiO}_{2}\right.$, petroleum ether/ethyl acetate $40 / 1$ to 10/1) yielded cyclopropane 3 (98 mg, $0.40 \mathrm{mmol}, 81 \%$ ) as a mixture of diastereomers, trans:cis-2.8:1. 


\section{trans-3}

colorless oil

${ }^{1} \mathrm{H}$ NMR $\left(400 \mathrm{MHz}, \mathrm{CDCl}_{3}\right), \delta(\mathrm{ppm}): 7.29(\mathrm{~m}, 2 \mathrm{H}), 7.21(\mathrm{~m}, 3 \mathrm{H}), 4.13(\mathrm{q}, J=7.1 \mathrm{~Hz}, 2 \mathrm{H}), 2.78(\mathrm{t}, J=$ $7.6 \mathrm{~Hz}, 2 \mathrm{H}), 2.41(\mathrm{t}, J=7.6 \mathrm{~Hz}, 2 \mathrm{H}), 1.81(\mathrm{~m}, 2 \mathrm{H}), 1.32(\mathrm{~m}, 1 \mathrm{H}), 1.27(\mathrm{t}, J=7.1 \mathrm{~Hz}, 3 \mathrm{H}), 1.09(\mathrm{~m}, 1$ $\mathrm{H}) ;{ }^{13} \mathrm{C}$ NMR (100 MHz, $\mathrm{CDCl}_{3}$ ), $\delta(\mathrm{ppm}): 172.6,140.7,128.4$ (2), 128.3 (2), 126.3, 80.6, 77.2, 60.8, 35.2, 22.7, 20.9, 16.8, 14.2, 10.6; IR (film) $v=3063$ (w), 3027 (w), 2981 (w), 2931 (w), 1722 (s), 1603 (w), $1495(\mathrm{w}), 1453(\mathrm{~m}), 1443(\mathrm{~m}), 1405(\mathrm{~m}), 1386(\mathrm{~m}), 1315(\mathrm{~m}), 1263(\mathrm{~m}), 1205(\mathrm{~m}), 1177(\mathrm{~s}), 1101$ (m), $1077(\mathrm{~m}), 1043(\mathrm{~m}), 1019(\mathrm{~m}), 927(\mathrm{~m}), 842(\mathrm{~m}), 746(\mathrm{~m}), 698(\mathrm{~s}), 576(\mathrm{w}), 515(\mathrm{w}), 490(\mathrm{~m})$; HRMS (ESI): calcd for $\left[\mathrm{C}_{16} \mathrm{H}_{18} \mathrm{O}_{2} \mathrm{Na}^{+}\right]$: 265.1199; found: 265.1197; $\boldsymbol{R}_{\mathbf{f}}=0.64$ (petroleum ether/ethyl acetate 10/1).

\section{cis-3}

colorless oil

${ }^{1} \mathbf{H}$ NMR $\left(400 \mathrm{MHz}, \mathrm{CDCl}_{3}\right), \delta(\mathrm{ppm}): 7.28(\mathrm{~m}, 2 \mathrm{H}), 7.20(\mathrm{~m}, 3 \mathrm{H}), 4.17$ (q, $\left.J=7.1 \mathrm{~Hz}, 2 \mathrm{H}\right), 2.79$ (t, $J=$ $7.7 \mathrm{~Hz}, 2 \mathrm{H}), 2.43(\mathrm{dt}, J=7.7,1.8 \mathrm{~Hz}, 2 \mathrm{H}), 1.88(\mathrm{~m}, 1 \mathrm{H}), 1.77(\mathrm{~m}, 1 \mathrm{H}), 1,37(\mathrm{~m}, 1 \mathrm{H}), 1.27(\mathrm{t}, J=7.1$ $\mathrm{Hz}, 3 \mathrm{H}), 1.15(\mathrm{~m}, 1 \mathrm{H}) ;{ }^{13} \mathrm{C}$ NMR $\left(100 \mathrm{MHz}, \mathrm{CDCl}_{3}\right) \delta(\mathrm{ppm}): 170.4,140.9,128.4$ (2), 128.3 (2), 126.2 , 79.1, 77.7, 60.7, 35.4, 21.1, 21.0, 14.4, 14.2, 9.7; IR (film) $v=3062$ (w), $3026(w), 2981(w), 2931(w)$, $1729(\mathrm{~s}), 1603(\mathrm{w}), 1496(\mathrm{~m}), 1454(\mathrm{~m}), 1401(\mathrm{~m}), 1380(\mathrm{~m}), 1354(\mathrm{~m}), 1272(\mathrm{~m}), 1177(\mathrm{~s}), 1146(\mathrm{~m})$, $1094(\mathrm{~m}), 1077(\mathrm{~m}), 1028(\mathrm{~m}), 947(\mathrm{w}), 897(\mathrm{w}), 860(\mathrm{~m}), 839(\mathrm{w}), 815(\mathrm{w}), 749(\mathrm{~m}), 698(\mathrm{~s}), 576(\mathrm{w})$, $498(\mathrm{~m})$; HRMS (ESI): calcd for $\left[\mathrm{C}_{16} \mathrm{H}_{18} \mathrm{O}_{2} \mathrm{Na}^{+}\right]$: 265.1199; found: 265.1196; $\boldsymbol{R}_{\mathrm{f}}=0.53$ (petroleum ether/ethyl acetate 10/1).

\section{ethyl 2-(phenylethynyl)cyclopropane-1-carboxylate 4}

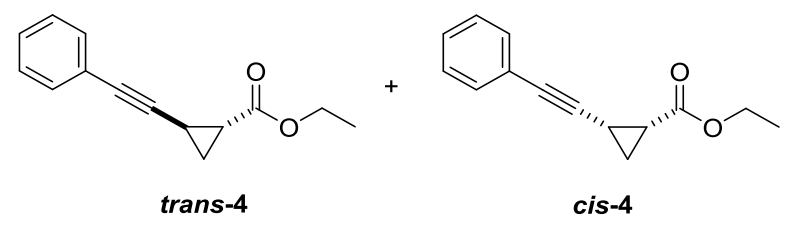

Thermal conditions:

According to GP-3, TBAFe (5.2 mg, $0.0125 \mathrm{mmol}, 2.5 \mathrm{~mol} \%)$, 4-nitroanisole $(0.38 \mathrm{mg}, 0.0025 \mathrm{mmol}$, $0.5 \mathrm{~mol} \%)$ in 1,2-DCE (250 $\mu \mathrm{L})$, but-3-en-1-yn-1-ylbenzene s1 (64.1 mg, $0.5 \mathrm{mmol}, 1$ eq.), and ethyl diazoacetate ( $63 \mu \mathrm{L}, 0.52 \mathrm{mmol}, 1.05$ eq.) were stirred at $40{ }^{\circ} \mathrm{C}$ for 24 hours. The reaction mixture was filtered through a plug of silica and eluted with $\mathrm{Et}_{2} \mathrm{O}$. Purification by column chromatography $\left(\mathrm{SiO}_{2}\right.$, petroleum ether/ethyl acetate $40 / 1$ to $10 / 1)$ yielded cyclopropane $4(57 \mathrm{mg}, 0.27 \mathrm{mmol}, 53 \%)$ as a mixture of diastereomers, trans:cis-2.2:1.

Photochemical conditions:

According to GP-4, TBAFe (10.3 mg, $0.025 \mathrm{mmol}, 5 \mathrm{~mol} \%)$, 1,4-dioxane (125 $\mu \mathrm{L})$, nitromethane (25 $\mu \mathrm{L}$ ), but-3-en-1-yn-1-ylbenzene s1 (64.1 mg, $0.5 \mathrm{mmol}, 1$ eq.), and ethyl diazoacetate (63 $\mu \mathrm{L}, 0.52$ $\mathrm{mmol}, 1.05$ eq.) were stirred at room temperature under irradiation for 20 hours. The reaction mixture was filtered through a plug of silica and eluted with $\mathrm{Et}_{2} \mathrm{O}$. The filtrate was concentrated under reduced pressure. Purification by column chromatography $\left(\mathrm{SiO}_{2}\right.$, petroleum ether/ethyl acetate $40 / 1$ to 10/1) yielded cyclopropane 4 (99 mg, $0.46 \mathrm{mmol}, 92 \%$ ) as a mixture of diastereomers, trans:cis-2.0:1. 


\section{trans-4}

colorless oil

${ }^{1}$ H NMR (400 MHz, $\mathrm{CDCl}_{3}$ ), $\delta$ (ppm): 7.34 (m, $2 \mathrm{H}$ ), 7.24 (m, 3H), 4.13 (q, $J=7.1 \mathrm{~Hz}, 2 \mathrm{H}$ ), 2.06-1.97 $(\mathrm{m}, 2 \mathrm{H}), 1.44(\mathrm{~m}, 1 \mathrm{H}), 1.26(\mathrm{~m}, 1 \mathrm{H}), 1.25(\mathrm{t}, J=7.1 \mathrm{~Hz}, 3 \mathrm{H}) ;{ }^{13} \mathrm{C} \mathrm{NMR}\left(100 \mathrm{MHz}, \mathrm{CDCl}_{3}\right), \delta(\mathrm{ppm})$ : 172.3, 131.7 (2), 128.2 (2), 128.0, 123.1, 89.5, 77.7, 61.0, 21.1, 17.1, 14.2, 11.0; IR (film) $v=2981$ (w), 2234 (w), 1721 (s), 1598 (w), $1572(w), 1492(\mathrm{~m}), 1441(\mathrm{~m}), 1405(\mathrm{~m}), 1387(\mathrm{~m}), 1323(\mathrm{~m}), 1264$ (m), $1192(\mathrm{~s}), 1176(\mathrm{~s}), 1116(\mathrm{w}), 1095(\mathrm{w}), 1055(\mathrm{~m}), 1034(\mathrm{~m}), 1000(\mathrm{w}), 986(\mathrm{~m}), 925(\mathrm{~m}), 892(\mathrm{w})$, $862(\mathrm{w}), 832(\mathrm{~m}), 754$ (s), 690 (s), 539 (m), 484 (w), 430 (w); MS (ESI): m/z (\%): 237 (100), 234 (48); HRMS (ESI): calcd for $\left[\mathrm{C}_{14} \mathrm{H}_{14} \mathrm{O}_{2} \mathrm{Na}^{+}\right]$: 237.0886; found: $237.0875 ; \boldsymbol{R}_{\mathfrak{f}}=0.55$ (petroleum ether/ethyl acetate 10/1).

The analytical data were consistent with those published in the literature ${ }^{[26]}$.

\section{cis-4}

colorless oil

${ }^{1}$ H NMR (400 MHz, $\mathrm{CDCl}_{3}$ ), $\delta(\mathrm{ppm}): 7.39(\mathrm{~m}, 2 \mathrm{H}), 7.27(\mathrm{~m}, 3 \mathrm{H}), 4.21(\mathrm{dq}, J=7.1,2.1 \mathrm{~Hz}, 2 \mathrm{H}), 2.03$ $(\mathrm{m}, 2 \mathrm{H}), 1.56(\mathrm{~m}, 1 \mathrm{H}), 1.30(\mathrm{~m}, 1 \mathrm{H}), 1.28(\mathrm{t}, J=7.1 \mathrm{~Hz}, 3 \mathrm{H}) ;{ }^{13} \mathrm{C} \mathrm{NMR}\left(100 \mathrm{MHz}, \mathrm{CDCl}_{3}\right), \delta(\mathrm{ppm})$ : 170.2, 131.7 (2), 128.1 (2), 127.7, 123.5, 87.2, 79.5, 60.8, 21.7, 14.6, 14.4, 10.0; IR (film) $v=2981$ (w), $2230(w), 1727(\mathrm{~s}), 1598(w), 1571(w), 1491(\mathrm{~m}), 1464(\mathrm{w}), 1442(\mathrm{~m}), 1401(\mathrm{~m}), 1381(\mathrm{~m}), 1354$ (w), $1279(w), 1257(w), 1179(\mathrm{~s}), 1112(\mathrm{w}), 1096(\mathrm{w}), 1068(\mathrm{w}), 1050(\mathrm{~m}), 1027(\mathrm{~m}), 979(\mathrm{w}), 916(\mathrm{w})$, 878 (w), 859 (w), 823 (w), 755 (s), 692 (s), 535 (m), 493 (w); MS (ESI): m/z (\%): 237 (100), 234 (13); HRMS (ESI): calcd for $\left[\mathrm{C}_{14} \mathrm{H}_{14} \mathrm{O}_{2} \mathrm{Na}^{+}\right]$: 237.0886; found: $237.0880 ; \boldsymbol{R}_{\mathfrak{f}}=0.42$ (petroleum ether/ethyl acetate 10/1).

The analytical data were consistent with those published in the literature ${ }^{[26]}$.

\section{ethyl 2-((4-bromophenyl)ethynyl)cyclopropane-1-carboxylate 5}

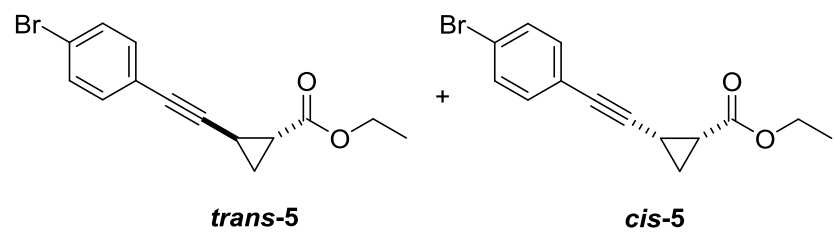

Thermal conditions:

According to GP-3, TBAFe (5.2 mg, $0.0125 \mathrm{mmol}, 2.5 \mathrm{~mol} \%)$, 4-nitroanisole (0.38 $\mathrm{mg}, 0.0025 \mathrm{mmol}$, $0.5 \mathrm{~mol} \%)$ in 1,2-DCE $(250 \mu \mathrm{L}), 1$-bromo-4-(but-3-en-1-yn-1-yl)benzene s3 (103.5 mg, $0.5 \mathrm{mmol}, 1$ eq.), and ethyl diazoacetate ( $63 \mu \mathrm{L}, 0.52 \mathrm{mmol}, 1.05$ eq.) were stirred at $40{ }^{\circ} \mathrm{C}$ for 24 hours. The reaction mixture was filtered through a plug of silica and eluted with EtOAc. Purification by column chromatography $\left(\mathrm{SiO}_{2}\right.$, petroleum ether/ethyl acetate 90/1 to 10/1) yielded cyclopropane 5 (125 mg, $0.43 \mathrm{mmol}, 85 \%)$ as a mixture of diastereomers, trans:cis-2.2:1.

\section{Photochemical conditions:}

According to GP-4, TBAFe (10.3 mg, $0.025 \mathrm{mmol}, 5 \mathrm{~mol} \%)$, 1,4-dioxane (125 $\mu \mathrm{L})$, nitromethane (25 $\mu \mathrm{L}$ ), 1-bromo-4-(but-3-en-1-yn-1-yl)benzene s3 (103.5 mg, $0.5 \mathrm{mmol}, 1$ eq.), and ethyl diazoacetate (63 $\mu \mathrm{L}, 0.52 \mathrm{mmol}, 1.05$ eq.) were stirred at room temperature under irradiation for 20 hours. The 
reaction mixture was filtered through a plug of silica and eluted with EtOAc. The filtrate was concentrated under reduced pressure. Purification by column chromatography $\left(\mathrm{SiO}_{2}\right.$, petroleum ether/ethyl acetate $40 / 1$ to $10 / 1$ ) yielded cyclopropane 5 (138 $\mathrm{mg}, 0.47 \mathrm{mmol}, 94 \%$ ) as a mixture of diastereomers, trans:cis-1.9:1.

\section{trans-5}

colorless solid

${ }^{1} \mathbf{H}$ NMR $\left(400 \mathrm{MHz}, \mathrm{CDCl}_{3}\right), \delta(\mathrm{ppm}): 7.40(\mathrm{~d}, J=8.6 \mathrm{~Hz}, 2 \mathrm{H}), 7.22(\mathrm{~d}, J=8.5 \mathrm{~Hz}, 2 \mathrm{H}), 4.16(\mathrm{q}, J=$ $7.1 \mathrm{~Hz}, 2 \mathrm{H}), 2.07-1.99(\mathrm{~m}, 2 \mathrm{H}), 1.47(\mathrm{~m}, 1 \mathrm{H}), 1.28(\mathrm{t}, J=7.1 \mathrm{~Hz}, 3 \mathrm{H}), 1.27(\mathrm{~m}, 1 \mathrm{H}) ;{ }^{13} \mathrm{C}$ NMR $(100$ $\left.\mathrm{MHz}, \mathrm{CDCl}_{3}\right), \delta(\mathrm{ppm}): 172.2,133.1$ (2), 131.5 (2), 122.2, 122.1, 90.8, 77.3, 61.0, 23.1, 17.0, 14.2, 10.9; IR (film) $v=2980$ (w), 2232 (w), 1900 (w), 1721 (s), 1586 (w), $1486(\mathrm{~s}), 1440$ (m), 1405 (m), $1387(\mathrm{~m}), 1322(\mathrm{~m}), 1263(\mathrm{~m}), 1176(\mathrm{~s}), 1114(\mathrm{w}), 1095(\mathrm{w}), 1070(\mathrm{~m}), 1054(\mathrm{~m}), 1033(\mathrm{~m}), 1010(\mathrm{~m})$, $988(\mathrm{~m}), 925(\mathrm{~m}), 893(\mathrm{w}), 821(\mathrm{~s}), 753(\mathrm{~m}), 706(\mathrm{~m}), 636(\mathrm{w}), 550(\mathrm{~m}), 525(\mathrm{~m}), 486(\mathrm{w}), 419(\mathrm{w})$; MS (ESI): $\mathrm{m} / \mathrm{z}$ (\%): 317 (100), 315 (98); HRMS (ESI): calcd for $\left[\mathrm{C}_{14} \mathrm{H}_{13} \mathrm{BrO}_{2} \mathrm{Na}^{+}\right]$: 314.9991; found: $314.9974 ; \boldsymbol{R}_{\mathfrak{f}}=0.52$ (petroleum ether/ethyl acetate $10 / 1$ ); Melting point: $36^{\circ} \mathrm{C}$.

The X-Ray structure of trans-5 is deposited at the Cambridge Crystallographic Data Centre (www.ccdc.cam.ac.uk) under CCDC 1913231.

\section{cis-5}

colorless oil

${ }^{1} \mathrm{H}$ NMR (400 MHz, $\left.\mathrm{CDCl}_{3}\right), \delta(\mathrm{ppm}): 7.39(\mathrm{~d}, J=8.5 \mathrm{~Hz}, 2 \mathrm{H}), 7.23(\mathrm{~d}, J=8.5 \mathrm{~Hz}, 2 \mathrm{H}), 4.19$ (dq, $J=$ 7.1, $1.0 \mathrm{~Hz}, 2 \mathrm{H}), 2.06-1.95(\mathrm{~m}, 2 \mathrm{H}), 1.53(\mathrm{~m}, 1 \mathrm{H}), 1.29(\mathrm{~m}, 1 \mathrm{H}), 1.26(\mathrm{t}, J=7.1 \mathrm{~Hz}, 3 \mathrm{H}) ;{ }^{13} \mathrm{C} \mathrm{NMR}$ (100 MHz, $\mathrm{CDCl}_{3}$ ), $\delta$ (ppm): 170.1, 133.2 (2), 131.4 (2), 122.5, 121.9, 88.6, 78.5, 60.8, 21.7, 14.6, 14.4, 9.9; IR (film) $v=2981$ (w), 2230 (w), 1727 (s), $1586(w), 1486(\mathrm{~m}), 1444(w), 1394(\mathrm{~m}), 1381$ (m), $1355(\mathrm{~m}), 1280(\mathrm{w}), 1258(\mathrm{w}), 1181(\mathrm{~s}), 1111(\mathrm{~m}), 1096(\mathrm{~m}), 1070(\mathrm{~m}), 1050(\mathrm{w}), 1028(\mathrm{~m}), 1010(\mathrm{~m})$, $980(w), 920(w), 878(w), 859(w), 823(s), 772(w), 713(w), 667(w), 559(w), 531(m), 520(w), 428$ (w); MS (ESI): $\mathrm{m} / z$ (\%): 317 (98), 315 (100); HRMS (ESI): calcd for [ $\left.\mathrm{C}_{14} \mathrm{H}_{13} \mathrm{BrO}_{2} \mathrm{Na}^{+}\right]: 314.9991$; found: 314.9995; $\boldsymbol{R}_{\mathrm{f}}=0.41$ (petroleum ether/ethyl acetate 10/1).

\section{ethyl 2-((4-methoxyphenyl)ethynyl)cyclopropane-1-carboxylate 6}

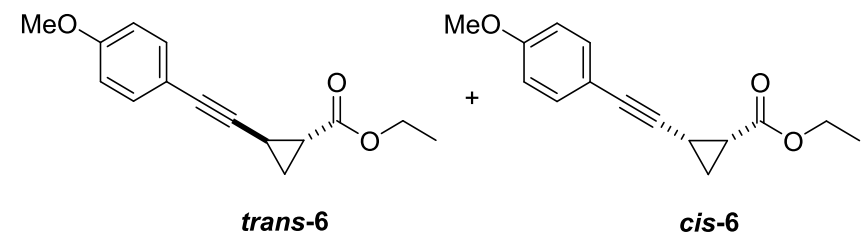

Thermal conditions:

According to GP-3, TBAFe (5.2 mg, $0.0125 \mathrm{mmol}, 2.5 \mathrm{~mol} \%)$, 4-nitroanisole (0.38 mg , $0.0025 \mathrm{mmol}$, $0.5 \mathrm{~mol} \%)$ in 1,2-DCE (250 $\mathrm{LL}), 1$-(but-3-en-1-yn-1-yl)-4-methoxybenzene s5 (79.1 mg, $0.5 \mathrm{mmol}, 1$ eq.), and ethyl diazoacetate ( $63 \mu \mathrm{L}, 0.52 \mathrm{mmol}, 1.05$ eq.) were stirred at $40{ }^{\circ} \mathrm{C}$ for 24 hours. The reaction mixture was filtered through a plug of silica and eluted with $\mathrm{Et}_{2} \mathrm{O}$. Purification by column chromatography $\left(\mathrm{SiO}_{2}\right.$, petroleum ether/ethyl acetate 40/1 to 20/1) yielded cyclopropane 6 (121 mg, $0.49 \mathrm{mmol}, 99 \%$ ) as a mixture of diastereomers, trans:cis-2.0:1. 


\section{Photochemical conditions:}

According to GP-4, TBAFe (10.3 mg, $0.025 \mathrm{mmol}, 5 \mathrm{~mol} \%)$, 1,4-dioxane (125 $\mu \mathrm{L})$, nitromethane (25 $\mu \mathrm{L}$ ), 1-(but-3-en-1-yn-1-yl)-4-methoxybenzene s5 (79.1 mg, $0.5 \mathrm{mmol}, 1$ eq.), and ethyl diazoacetate (63 $\mu \mathrm{L}, 0.52 \mathrm{mmol}, 1.05$ eq.) were stirred at room temperature under irradiation for 20 hours. The reaction mixture was filtered through a plug of silica and eluted with $\mathrm{Et}_{2} \mathrm{O}$. The filtrate was concentrated under reduced pressure. Purification by column chromatography $\left(\mathrm{SiO}_{2}\right.$, petroleum ether/ethyl acetate 40/1 to 20/1) yielded cyclopropane 6 (122 mg, $0.50 \mathrm{mmol}$, quant.) as a mixture of diastereomers, trans:cis-2.2:1.

\section{trans-6}

light yellow solid

${ }^{1} \mathbf{H}$ NMR $\left(400 \mathrm{MHz}, \mathrm{CDCl}_{3}\right), \delta(\mathrm{ppm}): 7.31(\mathrm{~d}, J=8.9 \mathrm{~Hz}, 2 \mathrm{H}), 6.80(\mathrm{~d}, J=8.9 \mathrm{~Hz}, 2 \mathrm{H}), 4.16(\mathrm{q}, J=$ $7.1 \mathrm{~Hz}, 2 \mathrm{H}), 3.79(\mathrm{~s}, 3 \mathrm{H}), 2.07-1.97(\mathrm{~m}, 2 \mathrm{H}), 1.45(\mathrm{~m}, 1 \mathrm{H}), 1.28(\mathrm{t}, J=7.1 \mathrm{~Hz}, 3 \mathrm{H}), 1.27(\mathrm{~m}, 1 \mathrm{H})$; ${ }^{13} \mathrm{C} \mathrm{NMR}\left(100 \mathrm{MHz}, \mathrm{CDCl}_{3}\right), \delta$ (ppm): 172.4, 159.4, 133.1 (2), 115.2, 113.9 (2), 88.0, 77.5, 60.9, 55.2, 23.1, 17.1, 14.2, 11.1; IR (film) $v=2980$ (w), 2936 (w), 2908 (w), 2837 (w), 1721 (s), 1605 (m), 1570 (w), 1509 (s), $1464(\mathrm{~m}), 1442(\mathrm{~m}), 1407(\mathrm{~m}), 1387(\mathrm{~m}), 1366(\mathrm{w}), 1323(\mathrm{~m}), 1288(\mathrm{~m}), 1244(\mathrm{~s}), 1193$ (s), $1170(\mathrm{~s}), 1107(\mathrm{~m}), 1055(\mathrm{~m}), 1031(\mathrm{~s}), 987(\mathrm{~m}), 926(\mathrm{~m}), 893(\mathrm{w}), 831(\mathrm{~s}), 793(\mathrm{~m}), 738(\mathrm{~m}), 686$ $(\mathrm{w}), 647(\mathrm{w}), 630(\mathrm{w}), 537(\mathrm{~m}), 475(\mathrm{w}) ;$ MS (ESI): $\mathrm{m} / \mathrm{z}(\%): 267$ (100); HRMS (ESI): calcd for $\left[\mathrm{C}_{15} \mathrm{H}_{16} \mathrm{O}_{3} \mathrm{Na}^{+}\right.$]: 267.0992; found: 267.1001; $\boldsymbol{R}_{\mathbf{f}}=0.43$ (petroleum ether/ethyl acetate 10/1); Melting point: $63^{\circ} \mathrm{C}$.

The X-Ray structure of trans-6 is deposited at the Cambridge Crystallographic Data Centre (www.ccdc.cam.ac.uk) under CCDC 1913243.

\section{cis-6}

light yellow oil

${ }^{1} \mathrm{H}$ NMR (400 MHz, $\left.\mathrm{CDCl}_{3}\right), \delta$ (ppm): 7.31 (d, $\left.J=8.9 \mathrm{~Hz}, 2 \mathrm{H}\right), 6.79(\mathrm{~d}, J=8.9 \mathrm{~Hz}, 2 \mathrm{H}), 4.19$ (dq, $J=$ 7.1, $1.2 \mathrm{~Hz}, 2 \mathrm{H}$ ), $3.78(\mathrm{~s}, 3 \mathrm{H}), 2.00(\mathrm{~m}, 2 \mathrm{H}), 1.52(\mathrm{~m}, 1 \mathrm{H}), 1.27(\mathrm{~m}, 1 \mathrm{H}), 1.26$ (t, $J=7.1 \mathrm{~Hz}, 3 \mathrm{H}$ ); ${ }^{13} \mathrm{C} \mathrm{NMR}\left(100 \mathrm{MHz}, \mathrm{CDCl}_{3}\right), \delta(\mathrm{ppm}): 170.2,159.2,133.1$ (2), 115.6, 113.8 (2), 85.6, 79.3, 60.7, 55.2, 21.7, 14.5, 14.4, 10.1; IR (film) $v=2980$ (w), 2937 (w), 2906 (w), 2837 (w), 1727 (s), 1605 (m), 1569 (w), $1508(\mathrm{~s}), 1464(\mathrm{~m}), 1442(\mathrm{~m}), 1402(\mathrm{~m}), 1381(\mathrm{~m}), 1354(\mathrm{w}), 1288(\mathrm{~m}), 1244(\mathrm{~s}), 1166(\mathrm{~s}), 1108$ (m), $1066(w), 1028(\mathrm{~s}), 981(\mathrm{w}), 920(\mathrm{w}), 878(\mathrm{w}), 859(\mathrm{w}), 831(\mathrm{~s}), 777(\mathrm{~m}), 648(\mathrm{w}), 631(\mathrm{w}), 538$ (m), 485 (w); MS (ESI): m/z (\%): 267 (100); HRMS (ESI): calcd for $\left[\mathrm{C}_{15} \mathrm{H}_{16} \mathrm{O}_{3} \mathrm{Na}^{+}\right]$: 267.0992 ; found: $267.1015 ; \boldsymbol{R}_{\mathfrak{f}}=0.28$ (petroleum ether/ethyl acetate 10/1).

\section{ethyl 2-((4-(dimethylamino)phenyl)ethynyl)cyclopropane-1-carboxylate 7}

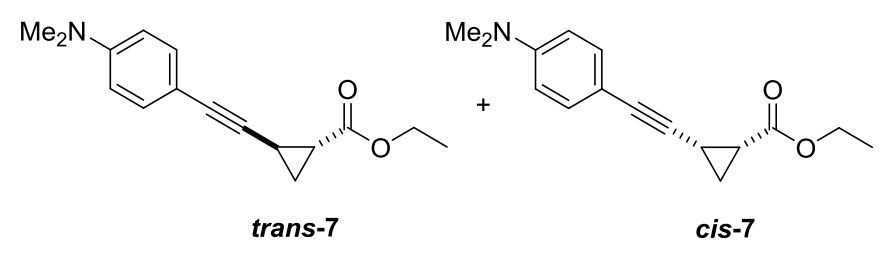

Thermal conditions: 
According to GP-3, TBAFe (5.2 mg, $0.0125 \mathrm{mmol}, 2.5 \mathrm{~mol} \%)$, 4-nitroanisole $(0.38 \mathrm{mg}, 0.0025 \mathrm{mmol}$, $0.5 \mathrm{~mol} \%$ ) in 1,2-DCE (250 $\mu \mathrm{L})$, 4-(but-3-en-1-yn-1-yl)- $N, N$-dimethylaniline s6 (85.6 mg, $0.5 \mathrm{mmol}, 1$ eq.), and ethyl diazoacetate ( $63 \mu \mathrm{L}, 0.52 \mathrm{mmol}, 1.05$ eq.) were stirred at $40{ }^{\circ} \mathrm{C}$ for 24 hours. The reaction mixture was filtered through a plug of silica and eluted with $\mathrm{Et}_{2} \mathrm{O}$. Purification by column chromatography $\left(\mathrm{SiO}_{2}\right.$, petroleum ether/ethyl acetate 20/1 to 10/1) yielded cyclopropane 7 (89 mg, $0.35 \mathrm{mmol}, 69 \%$ ) as a mixture of diastereomers, trans:cis-2.6:1.

Photochemical conditions:

According to GP-4, TBAFe (10.3 mg, $0.025 \mathrm{mmol}, 5 \mathrm{~mol} \%)$, 1,4-dioxane (125 $\mu \mathrm{L})$, nitromethane (25 $\mu \mathrm{L}$ ), 4-(but-3-en-1-yn-1-yl)- $N, N$-dimethylaniline $\mathbf{s} 6$ ( $85.6 \mathrm{mg}, 0.5 \mathrm{mmol}, 1$ eq.), and ethyl diazoacetate (63 $\mu \mathrm{L}, 0.52 \mathrm{mmol}, 1.05$ eq.) were stirred at room temperature under irradiation for 20 hours. The reaction mixture was filtered through a plug of silica and eluted with $\mathrm{Et}_{2} \mathrm{O}$. The filtrate was concentrated under reduced pressure. Purification by column chromatography $\left(\mathrm{SiO}_{2}\right.$, petroleum ether/ethyl acetate 20/1) yielded cyclopropane $7(116 \mathrm{mg}, 0.45 \mathrm{mmol}, 90 \%)$ as a mixture of diastereomers, trans:cis-2.2:1.

\section{trans-7}

orange oil

${ }^{1} \mathbf{H}$ NMR $\left(400 \mathrm{MHz}, \mathrm{CDCl}_{3}\right), \delta(\mathrm{ppm}): 7.25(\mathrm{~d}, J=8.9 \mathrm{~Hz}, 2 \mathrm{H}), 6.59(\mathrm{~d}, J=8.9 \mathrm{~Hz}, 2 \mathrm{H}), 4.15$ (q, $J=$ $7.1 \mathrm{~Hz}, 2 \mathrm{H}), 2.94(\mathrm{~s}, 6 \mathrm{H}), 2.05(\mathrm{~m}, 1 \mathrm{H}), 1.99(\mathrm{~m}, 1 \mathrm{H}), 1.44(\mathrm{~m}, 1 \mathrm{H}), 1.28(\mathrm{t}, J=7.1 \mathrm{~Hz}, 3 \mathrm{H}), 1.25$ $(\mathrm{m}, 1 \mathrm{H}) ;{ }^{13} \mathrm{C}$ NMR $\left(100 \mathrm{MHz}, \mathrm{CDCl}_{3}\right), \delta(\mathrm{ppm}): 172.5,149.9,132.7$ (2), $111.8(2), 110.0,86.9,78.5$, 60.8, $40.2(2), 23.2,17.2,14.2,11.3$; IR (film) $v=2980(w), 2899(w), 2802(w), 2225(w), 1720(s)$, $1606(\mathrm{~s}), 1519(\mathrm{~s}), 1479(\mathrm{w}), 1444(\mathrm{~m}), 1406(\mathrm{~m}), 1387(\mathrm{~m}), 1355(\mathrm{~m}), 1323(\mathrm{~m}), 1262(\mathrm{~m}), 1224(\mathrm{w})$, $1191(\mathrm{~s}), 1166(\mathrm{~s}), 1115(\mathrm{~m}), 1057(\mathrm{~m}), 1033(\mathrm{~m}), 1007(\mathrm{w}), 945(\mathrm{~m}), 926(\mathrm{~m}), 815(\mathrm{~s}), 767(\mathrm{~m}), 729$ (m), $684(w), 646(w), 617(w), 533(\mathrm{~m}), 488(\mathrm{~m}), 417(\mathrm{w})$; MS (ESI): $\mathrm{m} / \mathrm{z}(\%): 280(88), 258(100)$; HRMS (ESI): calcd for $\left[\mathrm{C}_{16} \mathrm{H}_{19} \mathrm{NO}_{2} \mathrm{Na}^{+}\right]$: 280.1308 ; found: $280.1307 ; \boldsymbol{R}_{\mathfrak{f}}=0.43$ (petroleum ether/ethyl acetate $5 / 1)$.

\section{cis-7}

orange solid

${ }^{1} \mathrm{H}$ NMR $\left(400 \mathrm{MHz}, \mathrm{CDCl}_{3}\right), \delta(\mathrm{ppm}): 7.24(\mathrm{~d}, J=8.9 \mathrm{~Hz}, 2 \mathrm{H}), 6.57$ (d, $\left.J=8.9 \mathrm{~Hz}, 2 \mathrm{H}\right), 4.18$ (dq, $J=$ 7.1, $1.6 \mathrm{~Hz}, 2 \mathrm{H}), 2.93(\mathrm{~s}, 6 \mathrm{H}), 1.98(\mathrm{~m}, 1 \mathrm{H}), 1.51(\mathrm{~m}, 1 \mathrm{H}), 1.44(\mathrm{~m}, 1 \mathrm{H}), 1.26(\mathrm{t}, J=7.1 \mathrm{~Hz}, 3 \mathrm{H})$, $1.24(\mathrm{~m}, 1 \mathrm{H}) ;{ }^{13} \mathrm{C}$ NMR $\left(100 \mathrm{MHz}, \mathrm{CDCl}_{3}\right), \delta(\mathrm{ppm}): 170.3,149.8,132.7$ (2), $111.8(2), 110.5,84.5$, 80.2, 60.7, 40.2 (2), 21.7, 14.5, 14.4, 10.3; IR (film) $v=2981$ (w), 2902 (w), 2804 (w), 2224 (w), 1727 (s), $1606(\mathrm{~s}), 1518(\mathrm{~s}), 1479(\mathrm{w}), 1445(\mathrm{~m}), 1401(\mathrm{~m}), 1379(\mathrm{~m}), 1351(\mathrm{~s}), 1282(\mathrm{w}), 1261(\mathrm{~m}), 1224$ (m), $1163(\mathrm{~s}), 1111(\mathrm{~m}), 1063(\mathrm{~m}), 1029(\mathrm{~m}), 980(\mathrm{w}), 945(\mathrm{~m}), 878(\mathrm{w}), 859(\mathrm{w}), 816(\mathrm{~s}), 773(\mathrm{w}), 645$ (w), $618(w), 535(\mathrm{~m}), 506(\mathrm{w})$; MS (ESI): m/z (\%): 280 (100), 258 (62); HRMS (ESI): calcd for $\left[\mathrm{C}_{16} \mathrm{H}_{19} \mathrm{NO}_{2} \mathrm{Na}^{+}\right]$: 280.1308; found: $280.1314 ; \boldsymbol{R}_{\mathfrak{f}}=0.28$ (petroleum ether/ethyl acetate 5/1); Melting point: $54^{\circ} \mathrm{C}$.

The X-Ray structure of cis-7 is deposited at the Cambridge Crystallographic Data Centre (www.ccdc.cam.ac.uk) under CCDC 1913244. 
ethyl 2-(p-tolylethynyl)cyclopropane-1-carboxylate 8

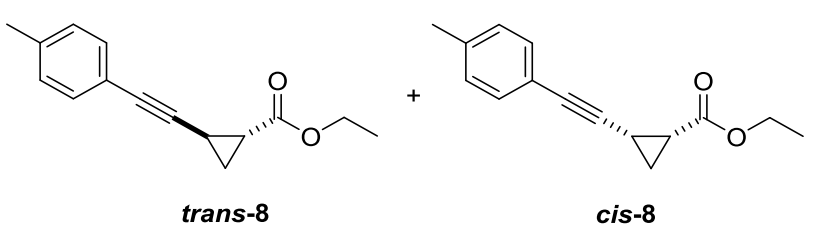

Thermal conditions:

According to GP-3, TBAFe (5.2 mg, $0.0125 \mathrm{mmol}, 2.5 \mathrm{~mol} \%)$, 4-nitroanisole $(0.38 \mathrm{mg}, 0.0025 \mathrm{mmol}$, $0.5 \mathrm{~mol} \%)$ in 1,2-DCE $(250 \mu \mathrm{L}), 1$-(but-3-en-1-yn-1-yl)-4-methylbenzene s8 $(71.1 \mathrm{mg}, 0.5 \mathrm{mmol}, 1$ eq.), and ethyl diazoacetate ( $63 \mu \mathrm{L}, 0.52 \mathrm{mmol}, 1.05$ eq.) were stirred at $40{ }^{\circ} \mathrm{C}$ for 24 hours. The reaction mixture was filtered through a plug of silica and eluted with $\mathrm{Et}_{2} \mathrm{O}$. Purification by column chromatography $\left(\mathrm{SiO}_{2}\right.$, petroleum ether/ethyl acetate 40/1 to 20/1) yielded cyclopropane 8 (97 mg, $0.43 \mathrm{mmol}, 85 \%$ ) as a mixture of diastereomers, trans:cis-1.9:1.

Photochemical conditions:

According to GP-4, TBAFe (10.3 mg, $0.025 \mathrm{mmol}, 5 \mathrm{~mol} \%)$, 1,4-dioxane (125 $\mu \mathrm{L})$, nitromethane (25 $\mu \mathrm{L}$ ), 1-(but-3-en-1-yn-1-yl)-4-methylbenzene $\mathbf{s} 8$ (71.1 mg, $0.5 \mathrm{mmol}, 1$ eq.), and ethyl diazoacetate (63 $\mu \mathrm{L}, 0.52 \mathrm{mmol}, 1.05$ eq.) were stirred at room temperature under irradiation for 20 hours. The reaction mixture was filtered through a plug of silica and eluted with $\mathrm{Et}_{2} \mathrm{O}$. The filtrate was concentrated under reduced pressure. Purification by column chromatography $\left(\mathrm{SiO}_{2}\right.$, petroleum ether/ethyl acetate $40 / 1$ to 20/1) yielded cyclopropane 8 (112 mg, $0.49 \mathrm{mmol}, 98 \%)$ as a mixture of diastereomers, trans:cis2.1:1.

\section{trans-8}

yellow oil

${ }^{1} \mathrm{H}$ NMR $\left(400 \mathrm{MHz}, \mathrm{CDCl}_{3}\right), \delta(\mathrm{ppm}): 7.24$ (d, $\left.J=8.0 \mathrm{~Hz}, 2 \mathrm{H}\right), 7.06$ (d, $\left.J=7.9 \mathrm{~Hz}, 2 \mathrm{H}\right), 4.14$ (q, $J=$ $7.1 \mathrm{~Hz}, 2 \mathrm{H}), 2.30(\mathrm{~s}, 3 \mathrm{H}), 2.06-1.96(\mathrm{~m}, 2 \mathrm{H}), 1.44(\mathrm{~m}, 1 \mathrm{H}), 1.26(\mathrm{t}, J=7.1 \mathrm{~Hz}, 3 \mathrm{H}), 1.25(\mathrm{~m}, 1 \mathrm{H})$; ${ }^{13} \mathrm{C} \mathrm{NMR}\left(100 \mathrm{MHz}, \mathrm{CDCl}_{3}\right), \delta(\mathrm{ppm}): 172.4,138.0,131.6(2), 129.0$ (2), 120.0, 88.7, 77.8, 61.0, 23.1, 21.4, 17.1, 14.2, 11.0; IR (film) $v=2981$ (w), 1723 (s), $1511(\mathrm{~m}), 1440(\mathrm{~m}), 1406(\mathrm{~m}), 1387$ (m), 1323 $(\mathrm{m}), 1263(\mathrm{~m}), 1193(\mathrm{~s}), 1176(\mathrm{~s}), 1116(\mathrm{w}), 1055(\mathrm{~m}), 1035(\mathrm{~m}), 989(\mathrm{w}), 926(\mathrm{w}), 893(\mathrm{w}), 837(\mathrm{~m})$, 816 (s), 739 (s), 709 (w), 476 (w); MS (ESI): m/z (\%): 251 (100); HRMS (ESI): calcd for $\left[\mathrm{C}_{15} \mathrm{H}_{16} \mathrm{O}_{2} \mathrm{Na}^{+}\right]$: 251.1043; found: $251.1045 ; \boldsymbol{R}_{\mathfrak{f}}=0.62$ (petroleum ether/ethyl acetate 10/1).

\section{cis-8}

colorless oil

${ }^{1} \mathrm{H}$ NMR $\left(400 \mathrm{MHz}, \mathrm{CDCl}_{3}\right), \delta(\mathrm{ppm}): 7.25$ (d, $\left.J=7.9 \mathrm{~Hz}, 2 \mathrm{H}\right), 7.04(\mathrm{~d}, J=7.9 \mathrm{~Hz}, 2 \mathrm{H}), 4.17$ (dq, $J=$ 7.1, $2.0 \mathrm{~Hz}, 2 \mathrm{H}), 2.30(\mathrm{~s}, 3 \mathrm{H}), 1.98(\mathrm{~m}, 2 \mathrm{H}), 1.51(\mathrm{~m}, 1 \mathrm{H}), 1.25(\mathrm{~m}, 1 \mathrm{H}), 1.24(\mathrm{t}, J=7.1 \mathrm{~Hz}, 3 \mathrm{H})$; ${ }^{13} \mathrm{C}$ NMR $\left(100 \mathrm{MHz}, \mathrm{CDCl}_{3}\right), \delta$ (ppm): 170.2, 137.7, 131.6 (2), 128.9 (2), 120.4, 86.4, 79.6, 60.7, 21.7, 21.4, 14.6, 14.4, 10.0; IR (film) $v=2981$ (w), $1728(\mathrm{~s}), 1510(\mathrm{~m}), 1445(\mathrm{~m}), 1402(\mathrm{~m}), 1381(\mathrm{~m}), 1354$ $(\mathrm{m}), 1280(\mathrm{~m}), 1258(\mathrm{w}), 1180(\mathrm{~s}), 1111(\mathrm{~m}), 1066(\mathrm{w}), 1049(\mathrm{~m}), 1029(\mathrm{~m}), 980(\mathrm{w}), 920(\mathrm{w}), 878(\mathrm{w})$, $860(\mathrm{w}), 816(\mathrm{~s}), 769(\mathrm{w}), 709(\mathrm{w}), 648(\mathrm{w}), 533(\mathrm{~m}), 487(\mathrm{w})$; HRMS (ESI): calcd for $\left[\mathrm{C}_{15} \mathrm{H}_{16} \mathrm{O}_{2} \mathrm{Na}^{+}\right]$: 251.1043; found: $251.1041 ; \boldsymbol{R}_{\mathfrak{f}}=0.49$ (petroleum ether/ethyl acetate 10/1). 


\section{ethyl 2-((4-cyanophenyl)ethynyl)cyclopropane-1-carboxylate 9}

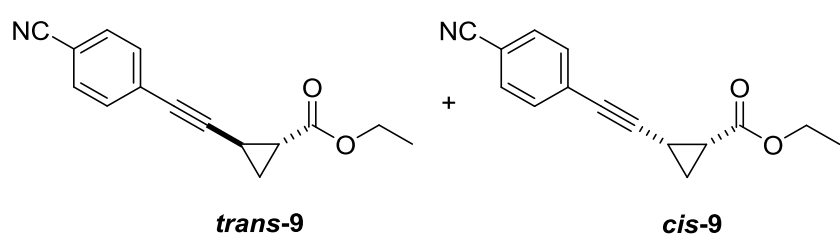

\section{Thermal conditions:}

According to GP-3, TBAFe (5.2 mg, $0.0125 \mathrm{mmol}, 2.5 \mathrm{~mol} \%)$, 4-nitroanisole (0.38 mg , $0.0025 \mathrm{mmol}$, $0.5 \mathrm{~mol} \%$ ) in 1,2-DCE (250 $\mu \mathrm{L}$ ), 4-(but-3-en-1-yn-1-yl)benzonitrile s10 (76.6 mg, $0.5 \mathrm{mmol}, 1$ eq.), and ethyl diazoacetate $\left(63 \mu \mathrm{L}, 0.52 \mathrm{mmol}, 1.05\right.$ eq.) were stirred at $40{ }^{\circ} \mathrm{C}$ for 24 hours. The reaction mixture was filtered through a plug of silica and eluted with $\mathrm{Et}_{2} \mathrm{O}$. Purification by column chromatography $\left(\mathrm{SiO}_{2}\right.$, petroleum ether/ethyl acetate 20/1 to 10/1) yielded cyclopropane 9 (96 mg, $0.40 \mathrm{mmol}, 80 \%$ ) as a mixture of diastereomers, trans:cis-1.8:1.

Photochemical conditions:

According to GP-4, TBAFe (10.3 mg, $0.025 \mathrm{mmol}, 5 \mathrm{~mol} \%)$, 1,4-dioxane (125 $\mu \mathrm{L})$, nitromethane (25 $\mu \mathrm{L}$ ), 4-(but-3-en-1-yn-1-yl)benzonitrile s10 (76.6 mg, $0.5 \mathrm{mmol}, 1$ eq.), and ethyl diazoacetate ( $63 \mu \mathrm{L}$, $0.52 \mathrm{mmol}, 1.05$ eq.) were stirred at room temperature under irradiation for 20 hours. The reaction mixture was filtered through a plug of silica and eluted with $\mathrm{Et}_{2} \mathrm{O}$. The filtrate was concentrated under reduced pressure. Purification by column chromatography $\left(\mathrm{SiO}_{2}\right.$, petroleum ether/ethyl acetate $20 / 1$ to 10/1) yielded cyclopropane 9 (98 $\mathrm{mg}, 0.41 \mathrm{mmol}, 82 \%)$ as a mixture of diastereomers, trans:cis-1.8:1.

\section{trans-9}

yellow oil

${ }^{1} \mathrm{H}$ NMR $\left(400 \mathrm{MHz}, \mathrm{CDCl}_{3}\right), \delta(\mathrm{ppm}): 7.56(\mathrm{~d}, J=8.5 \mathrm{~Hz}, 2 \mathrm{H}), 7.43(\mathrm{~d}, J=8.5 \mathrm{~Hz}, 2 \mathrm{H}), 4.17$ (q, $J=$ $7.1 \mathrm{~Hz}, 2 \mathrm{H}), 2.10-2.02(\mathrm{~m}, 2 \mathrm{H}), 1.50(\mathrm{~m}, 1 \mathrm{H}), 1.30(\mathrm{~m}, 1 \mathrm{H}), 1.28(\mathrm{t}, J=7.1 \mathrm{~Hz}, 3 \mathrm{H}) ;{ }^{13} \mathrm{C}$ NMR $(100$ $\left.\mathrm{MHz}, \mathrm{CDCl}_{3}\right), \delta(\mathrm{ppm}): 171.9,132.2$ (2), 131.9 (2), 128.2, 118.4, 111.3, 94.5, 76.4, 61.1, 23.2, 17.0, 14.2, 10.7; IR (film) $v=2982(\mathrm{w}), 2226$ (m), 1720 (s), 1603 (m), 1502 (m), 1465 (w), 1441 (m), 1406 $(\mathrm{m}), 1387(\mathrm{~m}), 1366(\mathrm{w}), 1324(\mathrm{~m}), 1267(\mathrm{~m}), 1193(\mathrm{~s}), 1177(\mathrm{~s}), 1106(\mathrm{w}), 1095(\mathrm{w}), 1083(\mathrm{w}), 1055$ (m), $1032(\mathrm{~m}), 986(\mathrm{~m}), 924(\mathrm{~m}), 894(\mathrm{w}), 834(\mathrm{~s}), 779(\mathrm{w}), 733(\mathrm{~m}), 684(\mathrm{w}), 554(\mathrm{~s}), 511(\mathrm{w}), 466(\mathrm{~m})$; MS (ESI): $\mathrm{m} / \mathrm{z}(\%): 262$ (100); HRMS (ESI): calcd for $\left[\mathrm{C}_{15} \mathrm{H}_{13} \mathrm{NO}_{2} \mathrm{Na}^{+}\right]: 262.0838$; found: 262.0836; $\boldsymbol{R}_{\mathbf{f}}$ $=0.34$ (petroleum ether/ethyl acetate $5 / 1)$.

\section{cis-9}

colorless solid

${ }^{1} \mathrm{H}$ NMR $\left(400 \mathrm{MHz}, \mathrm{CDCl}_{3}\right), \delta(\mathrm{ppm}): 7.54(\mathrm{~d}, J=8.5 \mathrm{~Hz}, 2 \mathrm{H}), 7.44(\mathrm{~d}, J=8.5 \mathrm{~Hz}, 2 \mathrm{H}), 4.20$ (q, $J=$ $7.1 \mathrm{~Hz}, 2 \mathrm{H}), 2.10-1.98(\mathrm{~m}, 2 \mathrm{H}), 1.55(\mathrm{~m}, 1 \mathrm{H}), 1.34(\mathrm{~m}, 1 \mathrm{H}), 1.27(\mathrm{t}, J=7.1 \mathrm{~Hz}, 3 \mathrm{H}) ;{ }^{13} \mathrm{C}$ NMR $(100$ $\mathrm{MHz}, \mathrm{CDCl}_{3}$ ), $\delta(\mathrm{ppm}): 169.9,132.2$ (2), 131.9 (2), 128.5, 118.6, 111.1, 92.5, 78.3, 60.9, 21.8, 14.8, 14.4, 9.9; IR (film) $v=2982(\mathrm{w}), 2225(\mathrm{~m}), 1726(\mathrm{~s}), 1603(\mathrm{~m}), 1502(\mathrm{~m}), 1464(\mathrm{w}), 1445(\mathrm{w}), 1403$ (m), $1381(\mathrm{~m}), 1354(\mathrm{~m}), 1273(\mathrm{~m}), 1259(\mathrm{~m}), 1181(\mathrm{~s}), 1109(\mathrm{~m}), 1066(\mathrm{w}), 1049(\mathrm{w}), 1027(\mathrm{~m}), 978$ (w), 920 (w), 879 (w), $840(\mathrm{~m}), 775$ (w), 654 (w), $556(\mathrm{~m}), 474(\mathrm{w})$; MS (ESI): m/z (\%): 262 (100); HRMS (ESI): calcd for $\left[\mathrm{C}_{15} \mathrm{H}_{13} \mathrm{NO}_{2} \mathrm{Na}^{+}\right]$: 262.0838; found: 262.0844; $\boldsymbol{R}_{\mathfrak{f}}=0.23$ (petroleum ether/ethyl acetate $5 / 1$ ); Melting point: $62^{\circ} \mathrm{C}$. 
The X-Ray structure of cis-9 is deposited at the Cambridge Crystallographic Data Centre (www.ccdc.cam.ac.uk) under CCDC 1913245.

ethyl 2-((4-nitrophenyl)ethynyl)cyclopropane-1-carboxylate 10

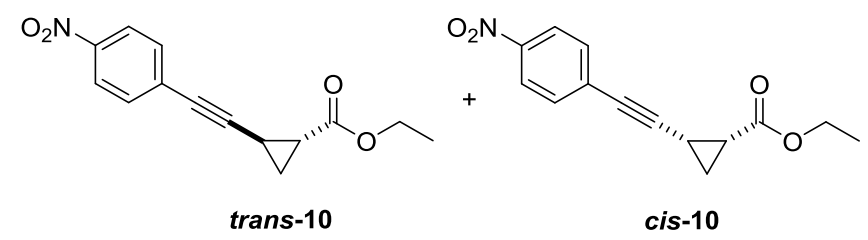

Thermal conditions:

According to GP-3, TBAFe (5.2 mg, $0.0125 \mathrm{mmol}, 2.5 \mathrm{~mol} \%)$, 4-nitroanisole $(0.38 \mathrm{mg}, 0.0025 \mathrm{mmol}$, $0.5 \mathrm{~mol} \%$ ) in 1,2-DCE (250 $\mathrm{LL}$ ), 1-(but-3-en-1-yn-1-yl)-4-nitrobenzene s12 (86.6 mg, 0.5 mmol, 1 eq.), and ethyl diazoacetate ( $63 \mu \mathrm{L}, 0.52 \mathrm{mmol}, 1.05$ eq.) were stirred at $40{ }^{\circ} \mathrm{C}$ for 24 hours. The reaction mixture was filtered through a plug of silica and eluted with $\mathrm{Et}_{2} \mathrm{O}$. Purification by column chromatography $\left(\mathrm{SiO}_{2}\right.$, petroleum ether/ethyl acetate 20/1 to 10/1) yielded cyclopropane 10 (130 mg, $0.50 \mathrm{mmol}, 94 \%$ ) as a mixture of diastereomers, trans:cis-2.1:1.

Photochemical conditions:

According to GP-4, TBAFe (10.3 mg, $0.025 \mathrm{mmol}, 5 \mathrm{~mol} \%)$, 1,4-dioxane $(125 \mu \mathrm{L})$, nitromethane $(25$ $\mu \mathrm{L}$ ), 1-(but-3-en-1-yn-1-yl)-4-nitrobenzene s12 (86.6 mg, $0.5 \mathrm{mmol}, 1$ eq.), and ethyl diazoacetate (63 $\mu \mathrm{L}, 0.52 \mathrm{mmol}, 1.05$ eq.) were stirred at room temperature under irradiation for 20 hours. The reaction mixture was filtered through a plug of silica and eluted with $\mathrm{Et}_{2} \mathrm{O}$. The filtrate was concentrated under reduced pressure. Purification by column chromatography $\left(\mathrm{SiO}_{2}\right.$, petroleum ether/ethyl acetate $20 / 1$ to 10/1) yielded cyclopropane 10 (120 mg, $0.47 \mathrm{mmol}$, quant.) as a mixture of diastereomers, trans:cis2.2:1.

\section{trans-10}

yellow oil

${ }^{1} \mathrm{H}$ NMR $\left(400 \mathrm{MHz}, \mathrm{CDCl}_{3}\right), \delta(\mathrm{ppm}): 8.15(\mathrm{~d}, J=8.9 \mathrm{~Hz}, 2 \mathrm{H}), 7.50(\mathrm{~d}, J=8.9 \mathrm{~Hz}, 2 \mathrm{H}), 4.18(\mathrm{q}, J=$ $7.1 \mathrm{~Hz}, 2 \mathrm{H}), 2.13-2.05(\mathrm{~m}, 2 \mathrm{H}), 1.53(\mathrm{~m}, 1 \mathrm{H}), 1.33(\mathrm{~m}, 1 \mathrm{H}), 1.29(\mathrm{t}, J=7.1 \mathrm{~Hz}, 3 \mathrm{H}) ;{ }^{13} \mathrm{C}$ NMR $(100$ $\left.\mathrm{MHz}, \mathrm{CDCl}_{3}\right), \delta(\mathrm{ppm}): 171.9,146.9,132.4(2), 130.2,123.5$ (2), 95.5, 76.2, 61.2, 23.3, 17.1, 14.2, 10.7; IR (film) $v=3106(w), 2982(w), 2232(m), 1725(s), 1594(m), 1518(s), 1493(w), 1441(w)$, $1408(\mathrm{~m}), 1387(\mathrm{w}), 1343(\mathrm{~s}), 1325(\mathrm{~m}), 1266(\mathrm{w}), 1196(\mathrm{~m}), 1181(\mathrm{~m}), 1108(\mathrm{w}), 1056(\mathrm{w}), 1033(\mathrm{w})$, 987 (w), 925 (w), $855(\mathrm{~m}), 750(\mathrm{~m}), 710$ (w), 689 (w), 524 (w); MS (ESI): m/z (\%): 282 (100); HRMS (ESI): calcd for $\left[\mathrm{C}_{14} \mathrm{H}_{13} \mathrm{NO}_{4} \mathrm{Na}^{+}\right]$: 282.0737 ; found: $282.0724 ; \boldsymbol{R}_{\mathbf{f}}=0.40$ (petroleum ether/ethyl acetate 10/1).

cis-10

yellow oil

${ }^{1} \mathrm{H}$ NMR $\left(400 \mathrm{MHz}, \mathrm{CDCl}_{3}\right), \delta(\mathrm{ppm}): 8.13(\mathrm{~d}, J=8.9 \mathrm{~Hz}, 2 \mathrm{H}), 7.51(\mathrm{~d}, J=8.9 \mathrm{~Hz}, 2 \mathrm{H}), 4.21$ (q, $J=$ $7.1 \mathrm{~Hz}, 2 \mathrm{H}), 2.13-1.99(\mathrm{~m}, 2 \mathrm{H}), 1.58(\mathrm{~m}, 1 \mathrm{H}), 1.37(\mathrm{~m}, 1 \mathrm{H}), 1.28(\mathrm{t}, J=7.1 \mathrm{~Hz}, 3 \mathrm{H}) ;{ }^{13} \mathrm{C}$ NMR $(100$ $\left.\mathrm{MHz}, \mathrm{CDCl}_{3}\right), \delta(\mathrm{ppm}): 169.9,146.8,132.4$ (2), 130.6, 123.5 (2), 93.6, 78.1, 61.0, 21.9, 14.9, 14.4, 9.9; 
IR (film) $v=3106(\mathrm{w}), 2983(\mathrm{w}), 2228(\mathrm{~m}), 1729(\mathrm{~s}), 1594(\mathrm{~m}), 1517(\mathrm{~s}), 1492(\mathrm{w}), 1403(\mathrm{~m}), 1381(\mathrm{~m})$, $1342(\mathrm{~s}), 1307(\mathrm{w}), 1284(\mathrm{w}), 1185(\mathrm{~s}), 1108(\mathrm{~m}), 1050(\mathrm{w}), 1028(\mathrm{w}), 978(\mathrm{w}), 854(\mathrm{~s}), 823(\mathrm{w}), 772$ (w), $750(\mathrm{~m}), 689(\mathrm{w}), 529(\mathrm{w})$; MS (ESI): $\mathrm{m} / \mathrm{z}(\%): 282(100)$; HRMS (ESI): calcd for $\left[\mathrm{C}_{14} \mathrm{H}_{13} \mathrm{NO}_{4} \mathrm{Na}^{+}\right]$: 282.0737; found: $282.0729 ; \boldsymbol{R}_{\mathfrak{f}}=0.26$ (petroleum ether/ethyl acetate 10/1).

ethyl 2-methyl-2-(phenylethynyl)cyclopropane-1-carboxylate 11

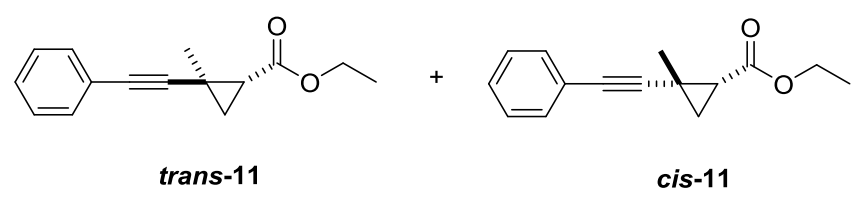

Thermal conditions:

According to GP-3, TBAFe (5.2 mg, $0.0125 \mathrm{mmol}, 2.5 \mathrm{~mol} \%)$, 4-nitroanisole $(0.38 \mathrm{mg}, 0.0025 \mathrm{mmol}$, $0.5 \mathrm{~mol} \%)$ in 1,2-DCE (250 $\mu \mathrm{L})$, (3-methylbut-3-en-1-yn-1-yl)benzene s13 (71.1 mg, $0.5 \mathrm{mmol}, 1$ eq.), and ethyl diazoacetate $\left(63 \mu \mathrm{L}, 0.52 \mathrm{mmol}, 1.05 \mathrm{eq}\right.$.) were stirred at $40{ }^{\circ} \mathrm{C}$ for 24 hours. The reaction mixture was filtered through a plug of silica and eluted with $\mathrm{Et}_{2} \mathrm{O}$. Purification by column chromatography $\left(\mathrm{SiO}_{2}\right.$, petroleum ether/ethyl acetate 40/1 to 10/1) yielded cyclopropane 11 (64 mg, $0.28 \mathrm{mmol}, 56 \%$ ) as a mixture of diastereomers, trans:cis-1.7:1.

Photochemical conditions:

According to GP-4, TBAFe (10.3 mg, $0.025 \mathrm{mmol}, 5 \mathrm{~mol} \%)$, 1,4-dioxane (125 $\mu \mathrm{L})$, nitromethane (25 $\mu \mathrm{L})$, (3-methylbut-3-en-1-yn-1-yl)benzene $\mathbf{s} 13(71.1 \mathrm{mg}, 0.5 \mathrm{mmol}, 1$ eq.), and ethyl diazoacetate (63 $\mu \mathrm{L}, 0.52 \mathrm{mmol}, 1.05$ eq.) were stirred at room temperature under irradiation for 20 hours. The reaction mixture was filtered through a plug of silica and eluted with $\mathrm{Et}_{2} \mathrm{O}$. The filtrate was concentrated under reduced pressure. Purification by column chromatography $\left(\mathrm{SiO}_{2}\right.$, petroleum ether/ethyl acetate $40 / 1$ to 10/1) yielded cyclopropane 11 (85 mg, $0.37 \mathrm{mmol}, 74 \%$ ) as a mixture of diastereomers, trans:cis1.8:1.

\section{trans-11}

colorless oil

${ }^{1} \mathbf{H}$ NMR (400 MHz, CDCl $), \delta(p p m): 7.35(\mathrm{~m}, 2 \mathrm{H}), 7.24(\mathrm{~m}, 3 \mathrm{H}), 4.16(\mathrm{q}, J=7.1 \mathrm{~Hz}, 2 \mathrm{H}), 2.13(\mathrm{~m}, 1$ $\mathrm{H}), 1.47(\mathrm{~s}, 3 \mathrm{H}), 1.42(\mathrm{~m}, 1 \mathrm{H}), 1.35(\mathrm{~m}, 1 \mathrm{H}), 1.27(\mathrm{t}, J=7.1 \mathrm{~Hz}, 3 \mathrm{H}) ;{ }^{13} \mathrm{C}$ NMR $\left(100 \mathrm{MHz}, \mathrm{CDCl}_{3}\right), \delta$ (ppm): 169.7, 130.6 (2), 127.2 (2), 126.8, 122.2, 93.1, 75.8, 59.8, 27.5, 21.5, 16.8, 16.2, 13.3; IR (film) $v=2979(w), 2936(w), 2223(w), 1722(s), 1597(w), 1492(w), 1443(m), 1401(m), 1382(m), 1357$ (w), $1314(\mathrm{~m}), 1266(\mathrm{w}), 1195(\mathrm{~s}), 1166(\mathrm{~s}), 1096(\mathrm{w}), 1067(\mathrm{~m}), 1021(\mathrm{w}), 979(\mathrm{w}), 957(\mathrm{w}), 936(\mathrm{w})$, $914(w), 858(w), 826(w), 776(m), 755(s), 691(m), 630(w), 547(w), 479(w) ; M S(E l, 70$ eV): m/z (\%): 228 (54), 199 (27), 183 (19), 171 (8), 155 (100), 141 (6), 128 (30), 115 (33), 105 (9), 77 (11); HRMS (El, $70 \mathrm{eV}$ ): calcd for $\left[\mathrm{C}_{15} \mathrm{H}_{16} \mathrm{O}_{2}\right]$ : 228.1150; found: $228.1151 ; \boldsymbol{R}_{\mathbf{f}}=0.58$ (petroleum ether/ethyl acetate 10/1).

cis-11

colorless oil 
${ }^{1} \mathbf{H}$ NMR (400 MHz, $\left.\mathrm{CDCl}_{3}\right), \delta(\mathrm{ppm}): 7.35(\mathrm{~m}, 2 \mathrm{H}), 7.23(\mathrm{~m}, 3 \mathrm{H}), 4.16(\mathrm{q}, J=7.2 \mathrm{~Hz}, 2 \mathrm{H}), 1.81(\mathrm{~m}, 1$ $\mathrm{H}), 1.68(\mathrm{~m}, 1 \mathrm{H}), 1.45(\mathrm{~s}, 3 \mathrm{H}), 1.24(\mathrm{t}, J=7.1 \mathrm{~Hz}, 3 \mathrm{H}), 1.11(\mathrm{~m}, 1 \mathrm{H}) ;{ }^{13} \mathbf{C ~ N M R}\left(100 \mathrm{MHz}, \mathrm{CDCl}_{3}\right), \delta$ (ppm): 170.2, 131.7 (2), 128.1 (2), 127.7, 123.5, 90.5, 79.4, 60.7, 29.8, 25.1, 22.3, 17.6, 14.4; IR (film) $v=2977(w), 2933(w), 2903(w), 2230(w), 1728(s), 1598(w), 1491(w), 1443(m), 1400(m), 1380$ $(\mathrm{m}), 1354(\mathrm{w}), 1339(\mathrm{w}), 1270(\mathrm{~m}), 1237(\mathrm{~m}), 1198(\mathrm{~m}), 1162(\mathrm{~s}), 1097(\mathrm{~m}), 1082(\mathrm{~m}), 1068(\mathrm{~m}), 1022$ (m), $968(w), 949(w), 934(w), 915(w), 881(w), 857(w), 822(w), 810(w), 786(w), 755(s), 691(s)$, $626(\mathrm{w}), 546(\mathrm{~m}), 513(\mathrm{w}), 483(\mathrm{w}), 453(\mathrm{w}), 429(\mathrm{w}) ;$ MS (El, $70 \mathrm{eV}): \mathrm{m} / \mathrm{z}(\%): 228(56), 199(28), 183$ (21), 171 (8), 155 (100), 141 (6), 128 (29), 115 (33), 105 (9), 77 (11); HRMS (El, 70 eV): calcd for $\left[\mathrm{C}_{15} \mathrm{H}_{16} \mathrm{O}_{2}\right]: 228.1150$; found: $228.1153 ; \boldsymbol{R}_{\mathrm{f}}=0.47$ (petroleum ether/ethyl acetate $10 / 1$ ).

ethyl 1-(phenylethynyl)-[1,1'-bi(cyclopropane)]-2-carboxylate 12

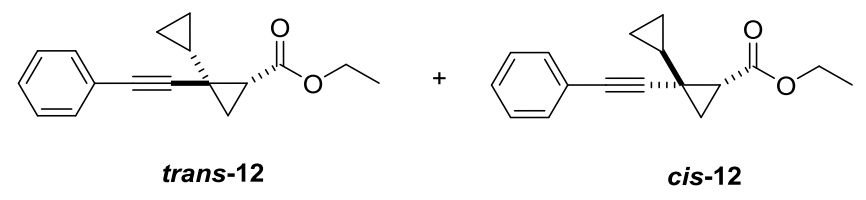

Thermal conditions:

According to GP-3, TBAFe (5.2 mg, $0.0125 \mathrm{mmol}, 2.5 \mathrm{~mol} \%)$, 4-nitroanisole (0.38 mg , $0.0025 \mathrm{mmol}$, $0.5 \mathrm{~mol} \%)$ in 1,2-DCE (250 $\mu \mathrm{L})$, 3-cyclopropylbut-3-en-1-yn-1-yl)benzene s15 (84.1 mg, $0.5 \mathrm{mmol}, 1$ eq.), and ethyl diazoacetate ( $63 \mu \mathrm{L}, 0.52 \mathrm{mmol}, 1.05$ eq.) were stirred at $40{ }^{\circ} \mathrm{C}$ for 24 hours. The reaction mixture was filtered through a plug of silica and eluted with $\mathrm{Et}_{2} \mathrm{O}$. Purification by column chromatography $\left(\mathrm{SiO}_{2}\right.$, petroleum ether/ethyl acetate 40/1 to 10/1) yielded cyclopropane 12 (118 mg, $0.46 \mathrm{mmol}, 93 \%$ ) as a mixture of diastereomers, trans:cis-1.9:1.

Photochemical conditions:

According to GP-4, TBAFe (10.3 mg, $0.025 \mathrm{mmol}, 5 \mathrm{~mol} \%)$, 1,4-dioxane $(125 \mu \mathrm{L})$, nitromethane $(25$ $\mu \mathrm{L}$ ), 3-cyclopropylbut-3-en-1-yn-1-yl)benzene s15 (84.1 mg, $0.5 \mathrm{mmol}, 1$ eq.), and ethyl diazoacetate (63 $\mu \mathrm{L}, 0.52 \mathrm{mmol}, 1.05$ eq.) were stirred at room temperature under irradiation for 20 hours. The reaction mixture was filtered through a plug of silica and eluted with $\mathrm{Et}_{2} \mathrm{O}$. The filtrate was concentrated under reduced pressure. Purification by column chromatography $\left(\mathrm{SiO}_{2}\right.$, petroleum ether/ethyl acetate $40 / 1$ to $10 / 1)$ yielded cyclopropane 12 (103 $\mathrm{mg}, 0.40 \mathrm{mmol}, 81 \%)$ as a mixture of diastereomers, trans:cis-2.3:1.

\section{trans-12}

colorless oil

${ }^{1} \mathbf{H}$ NMR $\left(400 \mathrm{MHz}, \mathrm{CDCl}_{3}\right), \delta(\mathrm{ppm}): 7.35(\mathrm{~m}, 2 \mathrm{H}), 7.27(\mathrm{~m}, 3 \mathrm{H}), 4.21(\mathrm{~m}, 2 \mathrm{H}), 2.24(\mathrm{~m}, 1 \mathrm{H}), 1.60$ $(\mathrm{m}, 1 \mathrm{H}), 1.51(\mathrm{~m}, 1 \mathrm{H}), 1.31(\mathrm{t}, J=7.1 \mathrm{~Hz}, 3 \mathrm{H}), 1.03(\mathrm{~m}, 1 \mathrm{H}), 0.74(\mathrm{~m}, 1 \mathrm{H}), 0.60-0.51(\mathrm{~m}, 2 \mathrm{H}), 0.49-$ $0.42(\mathrm{~m}, 1 \mathrm{H}) ;{ }^{13} \mathrm{C}$ NMR $\left(100 \mathrm{MHz}, \mathrm{CDCl}_{3}\right), \delta(\mathrm{ppm}): 170.5,131.7(2), 128.2(2), 127.9,123.1,90.1$, 78.6, 60.7, 29.4, 24.0, 22.2, 14.3, 10.7, 3.6, 2.8; IR (film) $v=3083(w), 2981(w), 2229(w), 1722(s)$, $1597(w), 1573(w), 1490(m), 1463(w), 1442(m), 1400(m), 1384(m), 1357(w), 1322(m), 1265(m)$, $1237(\mathrm{w}), 1197(\mathrm{~s}), 1163(\mathrm{~s}), 1095(\mathrm{~m}), 1039(\mathrm{~m}), 1023(\mathrm{~m}), 1000(\mathrm{~m}), 977(\mathrm{~m}), 945(\mathrm{w}), 913(\mathrm{~m}), 860$ 
(m), $837(\mathrm{~m}), 754(\mathrm{~s}), 690(\mathrm{~s}), 620(\mathrm{w}), 550(\mathrm{~m}), 521(\mathrm{w}), 491(\mathrm{w}), 414(\mathrm{w})$; HRMS (ESI): calcd for $\left[\mathrm{C}_{17} \mathrm{H}_{18} \mathrm{O}_{2} \mathrm{Na}^{+}\right]: 277.1199 ;$ found: $277.1199 ; \boldsymbol{R}_{\mathrm{f}}=0.56$ (petroleum ether/ethyl acetate 10/1).

cis-12

colorless oil

${ }^{1} \mathrm{H}$ NMR (400 MHz, $\left.\mathrm{CDCl}_{3}\right), \delta(\mathrm{ppm}): 7.37(\mathrm{~m}, 2 \mathrm{H}), 7.25(\mathrm{~m}, 3 \mathrm{H}), 4.18(\mathrm{q}, J=7.1 \mathrm{~Hz}, 2 \mathrm{H}), 1.91(\mathrm{~m}, 1$ $\mathrm{H}), 1.66(\mathrm{~m}, 1 \mathrm{H}), 1.26(\mathrm{t}, J=7.1 \mathrm{~Hz}, 3 \mathrm{H}), 1.14(\mathrm{~m}, 1 \mathrm{H}), 0.97(\mathrm{~m}, 1 \mathrm{H}), 0.59-0.41(\mathrm{~m}, 4 \mathrm{H}) ;{ }^{13} \mathbf{C} \mathbf{N M R}$ (100 MHz, $\mathrm{CDCl}_{3}$ ), $\delta$ (ppm): 170.0, 131.7 (2), 128.1 (2), 127.7, 123.5, 88.3, 80.3, 60.7, 28.7, 23.8, 19.9, 16.9, 14.4, 3.4, 3.0; IR (film) $v=3082(w), 2981(w), 2229(w), 1728(s), 1597(w), 1572(w)$, $1491(\mathrm{~m}), 1464(\mathrm{~m}), 1443(\mathrm{~m}), 1399(\mathrm{~m}), 1382(\mathrm{~m}), 1353(\mathrm{~m}), 1255(\mathrm{~m}), 1224(\mathrm{~m}), 1162(\mathrm{~s}), 1095(\mathrm{~m})$, $1069(\mathrm{~m}), 1034(\mathrm{~m}), 1000(\mathrm{w}), 946(\mathrm{w}), 911(\mathrm{w}), 842(\mathrm{~m}), 756(\mathrm{~s}), 692(\mathrm{~s}), 622(\mathrm{w}), 556(\mathrm{w}), 541(\mathrm{w})$, 521 (w), 491 (w); MS (El, $70 \mathrm{eV}$ ): m/z (\%): 168 (100), 152 (34), 139 (8), 127 (29), 115 (13), 102 (4), 89 (4), 77 (8); HRMS (El, $70 \mathrm{eV}$ ): calcd for $\left[\mathrm{C}_{17} \mathrm{H}_{18} \mathrm{O}_{2} \mathrm{Na}^{+}\right]$: 277.1199; found: $277.1199 ; \boldsymbol{R}_{\mathfrak{f}}=0.46$ (petroleum ether/ethyl acetate 10/1).

\section{ethyl 2-(3-hydroxy-3-phenylprop-1-yn-1-yl)cyclopropane-1-carboxylate 13}

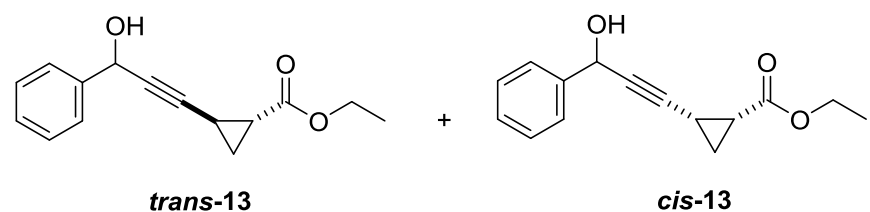

Photochemical conditions:

According to GP-4, TBAFe (10.3 mg, $0.025 \mathrm{mmol}, 5 \mathrm{~mol} \%)$, 1,4-dioxane (125 $\mu \mathrm{L})$, nitromethane (25 $\mu \mathrm{L})$, 1-phenylpent-4-en-2-yn-1-ol s16 (79.1 mg, $0.5 \mathrm{mmol}, 1$ eq.), and ethyl diazoacetate ( $63 \mu \mathrm{L}, 0.52$ $\mathrm{mmol}, 1.05$ eq.) were stirred at room temperature under irradiation for 20 hours. The reaction mixture was filtered through a plug of silica and eluted with EtOAc. The filtrate was concentrated under reduced pressure. Purification by column chromatography $\left(\mathrm{SiO}_{2}\right.$, petroleum ether/ethyl acetate $4 / 1$ to 1/1) yielded cyclopropane 13 (70 mg, $0.29 \mathrm{mmol}, 57 \%)$ as a mixture of diastereomers, trans:cis-1.5:1.

\section{trans-13}

yellow oil

${ }^{1} \mathrm{H}$ NMR (400 MHz, $\left.\mathrm{CDCl}_{3}\right), \delta(\mathrm{ppm}): 7.52(\mathrm{~m}, 2 \mathrm{H}), 7.32(\mathrm{~m}, 3 \mathrm{H}), 5.41(\mathrm{~s}, 1 \mathrm{H}), 4.13(\mathrm{~m}, 2 \mathrm{H}), 2.70(\mathrm{br}$ $\mathrm{s}, 1 \mathrm{H}), 1.96(\mathrm{~m}, 1 \mathrm{H}), 1.86(\mathrm{~m}, 1 \mathrm{H}), 1.45(\mathrm{~m}, 1 \mathrm{H}), 1.23(\mathrm{~m}, 1 \mathrm{H}), 1.22(\mathrm{dt}, J=7.1,1.8 \mathrm{~Hz}, 3 \mathrm{H}) ;{ }^{13} \mathrm{C}$ NMR (100 MHz, $\left.\mathrm{CDCl}_{3}\right), \delta(\mathrm{ppm}):$ 170.4, 141.0, 128.4 (2), 128.1, 126.7 (2), 84.3, 79.4, 64.6, 60.9, 21.3, 14.5, 14.3, 9.5; IR (film) $v=3429$ (m), 3062 (w), 2982 (w), 2245 (w), 1713 (s), 1602 (w), 1493 (w), $1452(\mathrm{~m}), 1404(\mathrm{~m}), 1381(\mathrm{~m}), 1354(\mathrm{~m}), 1275(\mathrm{~m}), 1181(\mathrm{~s}), 1146(\mathrm{~m}), 1094(\mathrm{~m}), 1026(\mathrm{~m}), 996$ $(\mathrm{m}), 943(\mathrm{w}), 919(\mathrm{w}), 901(\mathrm{w}), 861(\mathrm{~m}), 818(\mathrm{~m}), 764(\mathrm{~m}), 718(\mathrm{~m}), 698(\mathrm{~s}), 634(\mathrm{~m}), 570(\mathrm{w}), 542(\mathrm{~m})$; HRMS (ESI): calcd for $\left[\mathrm{C}_{15} \mathrm{H}_{16} \mathrm{O}_{3} \mathrm{Na}^{+}\right]$: 267.0992; found: 267.0991; $\boldsymbol{R}_{\boldsymbol{f}}=0.62$ (petroleum ether/ethyl acetate 2/1).

cis-13

yellow oil 
${ }^{1} \mathrm{H}$ NMR $\left(400 \mathrm{MHz}, \mathrm{CDCl}_{3}\right), \delta(\mathrm{ppm}): 7.50(\mathrm{~m}, 2 \mathrm{H}), 7.40-7.30(\mathrm{~m}, 3 \mathrm{H}), 5.42(\mathrm{~s}, 1 \mathrm{H}), 4.14$ (q, $J=7.1$ $\mathrm{Hz}, 2 \mathrm{H}), 2.12$ (br s, $1 \mathrm{H}), 1.94(\mathrm{~m}, 2 \mathrm{H}), 1.40(\mathrm{~m}, 1 \mathrm{H}), 1.27(\mathrm{dt}, J=7.1,1.8 \mathrm{~Hz}, 3 \mathrm{H}), 1.21(\mathrm{~m}, 1 \mathrm{H})$; ${ }^{13} \mathrm{C} \mathrm{NMR}\left(100 \mathrm{MHz}, \mathrm{CDCl}_{3}\right), \delta(\mathrm{ppm}): 172.2,140.7,128.6(2), 128.4,126.5$ (2), 86.8, 77.3, 64.7, 61.0, 22.8, 16.8, 14.2, 10.2; IR (film) $v=3429(\mathrm{~m}), 3064(w), 3031(w), 2982(w), 2937(w), 2239(w), 1723$ (s), $1706(\mathrm{~s}), 1602(\mathrm{w}), 1493(\mathrm{w}), 1449(\mathrm{~m}), 1408(\mathrm{~m}), 1387(\mathrm{~m}), 1317(\mathrm{~m}), 1268(\mathrm{~m}), 1208(\mathrm{~m}), 1182$ (s), $1098(\mathrm{w}), 1079(\mathrm{~m}), 1043(\mathrm{~m}), 1000(\mathrm{~m}), 926(\mathrm{~m}), 861(\mathrm{~m}), 843(\mathrm{~m}), 822(\mathrm{w}), 790(\mathrm{w}), 744(\mathrm{~m}), 713$ $(\mathrm{m}), 698(\mathrm{~m}), 634(\mathrm{~m}), 569(\mathrm{w}), 533(\mathrm{w}), 495(\mathrm{w}) ;$ MS (ESI): m/z (\%): 267 (100), 227 (17), 199 (75); HRMS (ESI): calcd for $\left[\mathrm{C}_{15} \mathrm{H}_{16} \mathrm{O}_{3} \mathrm{Na}^{+}\right]$: 267.0992; found: 267.0997; $\boldsymbol{R}_{\mathbf{f}}=0.48$ (petroleum ether/ethyl acetate 2/1).

\section{ethyl 2-(oct-1-yn-1-yl)cyclopropane-1-carboxylate 14}

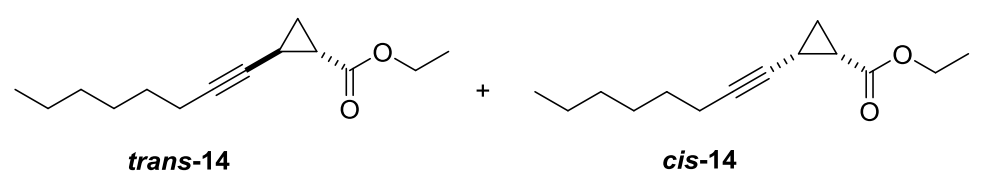

Thermal conditions:

According to GP-3, TBAFe (5.2 mg, $0.0125 \mathrm{mmol}, 2.5 \mathrm{~mol} \%)$, 4-nitroanisole (0.38 mg , $0.0025 \mathrm{mmol}$, $0.5 \mathrm{~mol} \%)$ in 1,2-DCE $(250 \mu \mathrm{L})$, dec-1-en-3-yne s19 (68.1 mg, $0.5 \mathrm{mmol}, 1$ eq.), and ethyl diazoacetate ( $63 \mu \mathrm{L}, 0.52 \mathrm{mmol}, 1.05$ eq.) were stirred at $40{ }^{\circ} \mathrm{C}$ for 24 hours. The reaction mixture was filtered through a plug of silica and eluted with $\mathrm{Et}_{2} \mathrm{O}$. Purification by column chromatography $\left(\mathrm{SiO}_{2}\right.$, petroleum ether/ethyl acetate 40/1 to 10/1) yielded cyclopropane 14 (63 mg, $0.28 \mathrm{mmol}, 57 \%$ ) as a mixture of diastereomers, trans:cis-2.7:1.

Photochemical conditions:

According to GP-4, TBAFe (10.3 mg, $0.025 \mathrm{mmol}, 5 \mathrm{~mol} \%)$, 1,4-dioxane (125 $\mu \mathrm{L})$, nitromethane (25 $\mu \mathrm{L}$ ), dec-1-en-3-yne $\mathbf{s} 19$ (68.1 mg, $0.5 \mathrm{mmol}, 1$ eq.), and ethyl diazoacetate $(63 \mu \mathrm{L}, 0.52 \mathrm{mmol}, 1.05$ eq.) were stirred at room temperature under irradiation for 20 hours. The reaction mixture was filtered through a plug of silica and eluted with $\mathrm{Et}_{2} \mathrm{O}$. The filtrate was concentrated under reduced pressure. Purification by column chromatography $\left(\mathrm{SiO}_{2}\right.$, petroleum ether/ethyl acetate $40 / 1$ to 10/1) yielded cyclopropane 14 (80 mg, $0.36 \mathrm{mmol}, 72 \%$ ) as a mixture of diastereomers, trans:cis-2.6:1.

\section{trans-14}

colorless oil

${ }^{1} \mathrm{H}$ NMR $\left(400 \mathrm{MHz}, \mathrm{CDCl}_{3}\right), \delta(\mathrm{ppm}): 4.12(\mathrm{q}, J=7.1 \mathrm{~Hz}, 2 \mathrm{H}), 2.10(\mathrm{dt}, J=7.0,1.7 \mathrm{~Hz}, 2 \mathrm{H}), 1.82(\mathrm{~m}$, $2 \mathrm{H}), 1.44(\mathrm{~m}, 2 \mathrm{H}), 1.37-1.24(\mathrm{~m}, 7 \mathrm{H}), 1.25(\mathrm{t}, J=7.1 \mathrm{~Hz}, 3 \mathrm{H}), 1.11(\mathrm{~m}, 1 \mathrm{H}), 0.88(\mathrm{t}, J=6.9 \mathrm{~Hz}, 3 \mathrm{H})$;

${ }^{13} \mathrm{C}$ NMR $\left(100 \mathrm{MHz}, \mathrm{CDCl}_{3}\right), \delta(\mathrm{ppm}): 172.6,79.7,78.1,60.8,31.3,28.8,28.5,22.7,22.5,18.7,16.9$, 14.2, 14.0, 10.7; IR (film) $v=2957(\mathrm{~m}), 2930(\mathrm{~m}), 2858(\mathrm{~m}), 1726(\mathrm{~s}), 1442(\mathrm{~m}), 1405(\mathrm{~m}), 1386(\mathrm{~m})$, $1313(\mathrm{~m}), 1261(\mathrm{~m}), 1205(\mathrm{~m}), 1177(\mathrm{~s}), 1101(\mathrm{w}), 1079(\mathrm{w}), 928(\mathrm{w}), 844(\mathrm{~m}), 769(\mathrm{w}), 752(\mathrm{w}), 726$ (w), 674 (w), 490 (w); MS (ESI): m/z (\%): 245 (100), 223 (18); HRMS (ESI): calcd for $\left[\mathrm{C}_{14} \mathrm{H}_{22} \mathrm{O}_{2} \mathrm{Na}^{+}\right]$: 245.1512; found: $245.1519 ; \boldsymbol{R}_{\mathfrak{f}}=0.60$ (petroleum ether/ethyl acetate $10 / 1$ ).

cis-14

colorless oil 
${ }^{1} \mathrm{H}$ NMR (400 MHz, $\mathrm{CDCl}_{3}$ ), $\delta(\mathrm{ppm}): 4.17$ (dq, $\left.J=7.1,1.5 \mathrm{~Hz}, 2 \mathrm{H}\right), 2.12(\mathrm{dt}, J=7.0,2.0 \mathrm{~Hz}, 2 \mathrm{H}$ ), $1.86(\mathrm{~m}, 1 \mathrm{H}), 1.76(\mathrm{~m}, 1 \mathrm{H}), 1.44(\mathrm{~m}, 2 \mathrm{H}), 1.38-1.24(\mathrm{~m}, 7 \mathrm{H}), 1.27(\mathrm{t}, J=7.1 \mathrm{~Hz}, 3 \mathrm{H}), 1.13(\mathrm{~m}, 1 \mathrm{H})$, $0.87(\mathrm{t}, J=7.0 \mathrm{~Hz}, 3 \mathrm{H}) ;{ }^{13} \mathrm{C}$ NMR $\left(100 \mathrm{MHz}, \mathrm{CDCl}_{3}\right), \delta(\mathrm{ppm}): 170.4,79.9,76.9,60.6,31.4,28.9$, 28.5, 22.5, 21.1, 18.8, 14.4, 14.1, 14.0, 9.7; IR (film) $v=2956(\mathrm{~m}), 2929(\mathrm{~m}), 2858(\mathrm{~m}), 1732(\mathrm{~s}), 1464$ $(\mathrm{m}), 1400(\mathrm{~m}), 1380(\mathrm{~m}), 1354(\mathrm{~m}), 1271(\mathrm{~m}), 1177(\mathrm{~s}), 1147(\mathrm{~m}), 1095(\mathrm{~m}), 1078(\mathrm{~m}), 1032(\mathrm{~m}), 932$ (w), 895 (w), 771 (w), 727 (w), 657 (w), 509 (w); MS (ESI): m/ z (\%): 245 (100), 223 (19); HRMS (ESI): calcd for $\left[\mathrm{C}_{14} \mathrm{H}_{22} \mathrm{O}_{2} \mathrm{Na}^{+}\right]$: 245.1512; found: $245.1507 ; \boldsymbol{R}_{\mathbf{f}}=0.50$ (petroleum ether/ethyl acetate 10/1).

ethyl 2-(3-acetoxy-3-phenylprop-1-yn-1-yl)cyclopropane-1-carboxylate 15

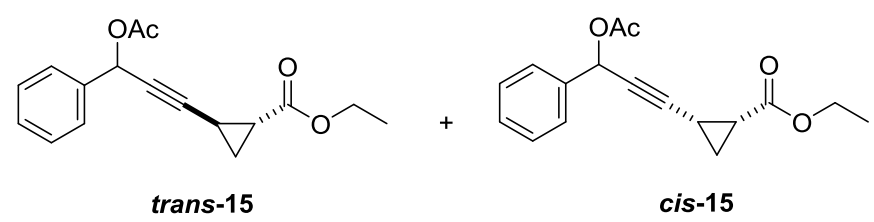

Thermal conditions:

According to GP-3, TBAFe (5.2 mg, $0.0125 \mathrm{mmol}, 2.5 \mathrm{~mol} \%)$, 4-nitroanisole $(0.38 \mathrm{mg}, 0.0025 \mathrm{mmol}$, $0.5 \mathrm{~mol} \%)$ in 1,2-DCE (250 $\mathrm{LL}), 1$-phenylpent-4-en-2-yn-1-yl acetate s17 (100.1 mg, $0.5 \mathrm{mmol}, 1$ eq.), and ethyl diazoacetate $\left(63 \mu \mathrm{L}, 0.52 \mathrm{mmol}, 1.05 \mathrm{eq}\right.$.) were stirred at $40{ }^{\circ} \mathrm{C}$ for 24 hours. The reaction mixture was filtered through a plug of silica and eluted with $\mathrm{Et}_{2} \mathrm{O}$. Purification by column chromatography $\left(\mathrm{SiO}_{2}\right.$, petroleum ether/ethyl acetate $10 / 1$ to $\left.5 / 1\right)$ yielded cyclopropane 15 (73 mg, $0.25 \mathrm{mmol}, 51 \%$ ) as a mixture of diastereomers, trans:cis-1.8:1.

Photochemical conditions:

According to GP-4, TBAFe (10.3 mg, $0.025 \mathrm{mmol}, 5 \mathrm{~mol} \%)$, 1,4-dioxane (125 $\mu \mathrm{L})$, nitromethane (25 $\mu \mathrm{L}$ ), 1-phenylpent-4-en-2-yn-1-yl acetate $\mathbf{s} 17$ (100.1 mg, $0.5 \mathrm{mmol}, 1$ eq.), and ethyl diazoacetate (63 $\mu \mathrm{L}, 0.52 \mathrm{mmol}, 1.05$ eq.) were stirred at room temperature under irradiation for 20 hours. The reaction mixture was filtered through a plug of silica and eluted with EtOAc. The filtrate was concentrated under reduced pressure. Purification by column chromatography $\left(\mathrm{SiO}_{2}\right.$, petroleum ether/ethyl acetate $10 / 1$ to 2/1) yielded cyclopropane 15 (107 mg, $0.37 \mathrm{mmol}, 75 \%)$ as a mixture of diastereomers, trans:cis$1.7: 1$.

\section{trans-15}

colorless oil

${ }^{1} \mathrm{H}$ NMR $\left(400 \mathrm{MHz}, \mathrm{CDCl}_{3}\right), \delta(\mathrm{ppm}): 7.47(\mathrm{~m}, 2 \mathrm{H}), 7.40-7.34(\mathrm{~m}, 3 \mathrm{H}), 6.41(\mathrm{~d}, J=1.6 \mathrm{~Hz}, 1 \mathrm{H}), 4.14$ (q, $J=7.1 \mathrm{~Hz}, 2 \mathrm{H}), 2.09(\mathrm{~s}, 3 \mathrm{H}), 1.94(\mathrm{~m}, 2 \mathrm{H}), 1.40(\mathrm{~m}, 1 \mathrm{H}), 1.27(\mathrm{t}, J=7.1 \mathrm{~Hz}, 3 \mathrm{H}), 1.21(\mathrm{~m}, 1 \mathrm{H})$; ${ }^{13} \mathrm{C}$ NMR (125 MHz, $\mathrm{CDCl}_{3}$ ), $\delta$ (ppm): 172.1, 169.8, 137.2, 128.9, 128.7 (2), 127.6 (2), 87.4, 74.1, 65.8, 61.0, 22.8, 21.1, 16.8, 14.2, 10.2; IR (film) $v=2982(w), 2245(w), 1723(s), 1495(w), 1443(\mathrm{~m})$, $1407(\mathrm{~m}), 1387(\mathrm{~m}), 1369(\mathrm{~m}), 1317(\mathrm{~m}), 1266(\mathrm{w}), 1222(\mathrm{~s}), 1179(\mathrm{~s}), 1097(\mathrm{w}), 1077(\mathrm{w}), 1043(\mathrm{~m})$, $1015(\mathrm{~m}), 958(\mathrm{~m}), 900(\mathrm{w}), 862(\mathrm{w}), 844(\mathrm{~m}), 793(\mathrm{w}), 756(\mathrm{w}), 721(\mathrm{w}), 697(\mathrm{~s}), 627(\mathrm{w}), 603(\mathrm{w}), 558$ (m), 534 (w), 499 (w); MS (ESI): m/z (\%): 309 (100), 249 (25), 227 (80), 199 (75); HRMS (ESI): calcd for $\left[\mathrm{C}_{17} \mathrm{H}_{18} \mathrm{O}_{4} \mathrm{Na}^{+}\right]$: 309.1097; found: 309.1102; $\boldsymbol{R}_{\mathfrak{f}}=0.43$ (petroleum ether/ethyl acetate 5/1). 
light green oil

${ }^{1} \mathrm{H}$ NMR $\left(400 \mathrm{MHz}, \mathrm{CDCl}_{3}\right), \delta(\mathrm{ppm}): 7.51(\mathrm{~m}, 2 \mathrm{H}), 7.38-7.30(\mathrm{~m}, 3 \mathrm{H}), 6.43(\mathrm{~d}, J=1.4 \mathrm{~Hz}, 1 \mathrm{H}), 4.13$ $(\mathrm{m}, 2 \mathrm{H}), 2.07(\mathrm{~d}, J=0.6 \mathrm{~Hz}, 3 \mathrm{H}), 1.96(\mathrm{~m}, 1 \mathrm{H}), 1.86(\mathrm{~m}, 1 \mathrm{H}), 1.49(\mathrm{~m}, 1 \mathrm{H}), 1.22(\mathrm{~m}, 1 \mathrm{H}), 1.21$ (dt, $J=7.1,4.5 \mathrm{~Hz}, 3 \mathrm{H}) ;{ }^{13} \mathrm{C}$ NMR $\left(125 \mathrm{MHz}, \mathrm{CDCl}_{3}\right), \delta(\mathrm{ppm}): 170.0,169.8,137.3,128.8,128.5(2)$, 127.9, 127.8, 85.1, 75.8, 65.9, 60.8, 21.4, 21.1, 14.3 (2), 9.3; IR (film) $v=2982$ (w), 2251 (w), 1729 (s), $1495(\mathrm{w}), 1455(\mathrm{~m}), 1402(\mathrm{~m}), 1369(\mathrm{~m}), 1273(\mathrm{~m}), 1223(\mathrm{~s}), 1179(\mathrm{~s}), 1095(\mathrm{~m}), 1015(\mathrm{~m}), 954(\mathrm{~m})$, $898(\mathrm{~m}), 863(\mathrm{~m}), 819(\mathrm{w}), 762(\mathrm{~m}), 731(\mathrm{~m}), 698(\mathrm{~m}), 627(\mathrm{w}), 603(\mathrm{w}), 559(\mathrm{~m}), 542(\mathrm{~m}), 514(\mathrm{w}), 466$ (w); MS (ESI): m/z (\%): 309 (100), 227 (88), 199 (32); HRMS (ESI): calcd for $\left[\mathrm{C}_{17} \mathrm{H}_{18} \mathrm{O}_{4} \mathrm{Na}^{+}\right]$: 309.1097; found: $309.1092 ; \boldsymbol{R}_{\mathrm{f}}=0.28$ (petroleum ether/ethyl acetate $5 / 1$ ).

\section{ethyl 2-(3-((tert-butyldiphenylsilyl)oxy)prop-1-yn-1-yl)cyclopropane-1-carboxylate 16}

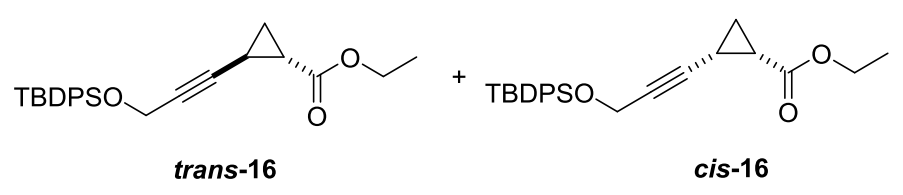

Thermal conditions:

According to GP-3, TBAFe (5.2 mg, $0.0125 \mathrm{mmol}, 2.5 \mathrm{~mol} \%)$, 4-nitroanisole $(0.38 \mathrm{mg}, 0.0025 \mathrm{mmol}$, $0.5 \mathrm{~mol} \%)$ in 1,2-DCE $(250 \mu \mathrm{L})$, tert-butyl(pent-4-en-2-yn-1-yloxy)diphenylsilane s20 (160.3 mg, 0.5 mmol, 1 eq.), and ethyl diazoacetate ( $63 \mu \mathrm{L}, 0.52 \mathrm{mmol}, 1.05$ eq.) were stirred at $40^{\circ} \mathrm{C}$ for 24 hours. The reaction mixture was filtered through a plug of silica and eluted with $\mathrm{Et}_{2} \mathrm{O}$. Purification by column chromatography $\left(\mathrm{SiO}_{2}\right.$, petroleum ether/ethyl acetate 40/1 to 20/1) yielded cyclopropane 16 (77 mg, $0.19 \mathrm{mmol}, 38 \%$ ) as a mixture of diastereomers, trans:cis-2.4:1.

Photochemical conditions:

According to GP-4, TBAFe (10.3 mg, $0.025 \mathrm{mmol}, 5 \mathrm{~mol} \%)$, 1,4-dioxane (125 $\mu \mathrm{L})$, nitromethane (25 $\mu \mathrm{L})$, tert-butyl(pent-4-en-2-yn-1-yloxy)diphenylsilane s20 (160.3 mg, $0.5 \mathrm{mmol}, 1$ eq.), and ethyl diazoacetate ( $63 \mu \mathrm{L}, 0.52 \mathrm{mmol}, 1.05$ eq.) were stirred at room temperature under irradiation for 20 hours. The reaction mixture was filtered through a plug of silica and eluted with EtOAc. The filtrate was concentrated under reduced pressure. Purification by column chromatography $\left(\mathrm{SiO}_{2}\right.$, petroleum ether/ethyl acetate $40 / 1$ to $20 / 1$ ) yielded cyclopropane 16 (108 mg, $0.26 \mathrm{mmol}, 53 \%$ ) as a mixture of diastereomers, trans:cis-2.3:1.

\section{trans-16}

colorless oil

${ }^{1} \mathrm{H}$ NMR $\left(300 \mathrm{MHz}, \mathrm{CDCl}_{3}\right), \delta(\mathrm{ppm}): 7.69(\mathrm{~m}, 4 \mathrm{H}), 7.47-7.36(\mathrm{~m}, 6 \mathrm{H}), 4.28(\mathrm{~d}, J=1.7 \mathrm{~Hz}, 2 \mathrm{H}), 4.14$ (q, $J=7.1 \mathrm{~Hz}, 2 \mathrm{H}), 1.79(\mathrm{~m}, 2 \mathrm{H}), 1.30(\mathrm{~m}, 1 \mathrm{H}), 1.26(\mathrm{t}, J=7.1 \mathrm{~Hz}, 3 \mathrm{H}), 1.05(\mathrm{~s}, 9 \mathrm{H}), 1.04(\mathrm{~m}, 1 \mathrm{H})$; ${ }^{13} \mathrm{C}$ NMR $\left(75 \mathrm{MHz}, \mathrm{CDCl}_{3}\right), \delta(\mathrm{ppm}): 172.3,135.6,133.2,129.8,127.7,85.2,75.8,60.9,52.8,26.7$, 22.6, 19.1, 16.6, 14.2, 10.3; IR (film) $v=3071(w), 2958(w), 2931(\mathrm{~m}), 2895(\mathrm{w}), 2858(\mathrm{~m}), 2243(\mathrm{w})$, $1726(\mathrm{~s}), 1589(\mathrm{w}), 1472(\mathrm{~m}), 1442(\mathrm{~m}), 1428(\mathrm{~m}), 1407(\mathrm{~m}), 1387(\mathrm{~m}), 1315(\mathrm{~m}), 1262(\mathrm{~m}), 1207(\mathrm{~m})$, $1779(\mathrm{~s}), 1108(\mathrm{~s}), 1068(\mathrm{~s}), 1009(\mathrm{~m}), 997(\mathrm{~m}), 927(\mathrm{~m}), 845(\mathrm{~m}), 823(\mathrm{~m}), 771(\mathrm{~m}), 738(\mathrm{~m}), 700(\mathrm{~s})$, $611(\mathrm{~m}), 504$ (s), 489 (s), 426 (w); HRMS (ESI): calcd for $\left[\mathrm{C}_{25} \mathrm{H}_{30} \mathrm{O}_{3} \mathrm{SiNa}^{+}\right]$: 429.1856; found: 429.1842; $\boldsymbol{R}_{\mathbf{f}}=0.63$ (petroleum ether/ethyl acetate $5 / 1$ ). 
cis-16

colorless oil

${ }^{1} \mathbf{H}$ NMR $\left(300 \mathrm{MHz}, \mathrm{CDCl}_{3}\right), \delta(\mathrm{ppm}): 7.70(\mathrm{~m}, 4 \mathrm{H}), 7.43-7.35(\mathrm{~m}, 6 \mathrm{H}), 4.28(\mathrm{~d}, J=2.0 \mathrm{~Hz}, 2 \mathrm{H}), 4.15$ (dq, $J=7.1,0.6 \mathrm{~Hz}, 2 \mathrm{H}), 1.91(\mathrm{~m}, 1 \mathrm{H}), 1.79(\mathrm{~m}, 1 \mathrm{H}), 1.39(\mathrm{~m}, 1 \mathrm{H}), 1.24(\mathrm{t}, J=7.1 \mathrm{~Hz}, 3 \mathrm{H}), 1.18(\mathrm{~m}, 1$ $\mathrm{H}), 1.05$ (s, $9 \mathrm{H}) ;{ }^{13} \mathrm{C}$ NMR (100 MHz, $\left.\mathrm{CDCl}_{3}\right), \delta$ (ppm): 170.1, 135.6, 133.3, 133.2, 129.7, 127.7, 82.4, 77.7, 60.7, 52.9, 26.7, 21.2, 19.2, 14.4, 14.1, 9.5; IR (film) $v=3071(\mathrm{w}), 3048(\mathrm{w}), 2957(\mathrm{~m}), 2930(\mathrm{~m})$, $2858(\mathrm{~m}), 2250(\mathrm{w}), 1733(\mathrm{~s}), 1598(\mathrm{w}), 1472(\mathrm{~m}), 1428(\mathrm{~m}), 1378(\mathrm{~m}), 1269(\mathrm{w}), 1181(\mathrm{~s}), 1108(\mathrm{~s})$, $1070(\mathrm{~s}), 1030(\mathrm{~m}), 998(\mathrm{~m}), 940(\mathrm{w}), 897(\mathrm{w}), 853(\mathrm{w}), 823(\mathrm{~m}), 741(\mathrm{~m}), 702(\mathrm{~s}), 613(\mathrm{~m}), 505(\mathrm{~s})$, $490(\mathrm{~m}), 442(\mathrm{w})$; HRMS (ESI): calcd for $\left[\mathrm{C}_{25} \mathrm{H}_{30} \mathrm{O}_{3} \mathrm{SiNa}^{+}\right]$: 429.1856; found: 429.1863; $\boldsymbol{R}_{\mathrm{f}}=0.54$ (petroleum ether/ethyl acetate 5/1).

ethyl 2-(3-((N-allyl-4-methylphenyl)sulfonamido)prop-1-yn-1-yl)cyclopropane-1-carboxylate 17

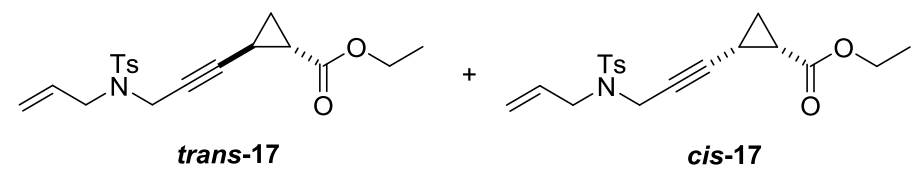

Thermal conditions:

According to GP-3, TBAFe (5.2 mg, $0.0125 \mathrm{mmol}, 2.5 \mathrm{~mol} \%)$, 4-nitroanisole $(0.38 \mathrm{mg}, 0.0025 \mathrm{mmol}$, $0.5 \mathrm{~mol} \%)$ in 1,2-DCE $(250 \mu \mathrm{L}), \mathrm{N}$-allyl-4-methyl- $N$-(pent-4-en-2-yn-1-yl)benzenesulfonamide s22 (137.7 mg, $0.5 \mathrm{mmol}, 1$ eq.), and ethyl diazoacetate (63 $\mu \mathrm{L}, 0.52 \mathrm{mmol}, 1.05$ eq.) were stirred at $40{ }^{\circ} \mathrm{C}$ for 24 hours. The reaction mixture was filtered through a plug of silica and eluted with $\mathrm{Et}_{2} \mathrm{O}$. Purification by column chromatography $\left(\mathrm{SiO}_{2}\right.$, petroleum ether/ethyl acetate $10 / 1$ to $\left.5 / 1\right)$ yielded cyclopropane 17 (49 mg, $0.13 \mathrm{mmol}, 27 \%$ ) as a mixture of diastereomers, trans:cis-2.0:1.

Photochemical conditions:

According to GP-4, TBAFe (10.3 mg, $0.025 \mathrm{mmol}, 5 \mathrm{~mol} \%)$, 1,4-dioxane (125 $\mu \mathrm{L})$, nitromethane (25 $\mu \mathrm{L}$ ), $N$-allyl-4-methyl- $N$-(pent-4-en-2-yn-1-yl)benzenesulfonamide s22 (137.7 mg, $0.5 \mathrm{mmol}, 1$ eq.), and ethyl diazoacetate ( $63 \mu \mathrm{L}, 0.52 \mathrm{mmol}, 1.05 \mathrm{eq}$.) were stirred at room temperature under irradiation for 20 hours. The reaction mixture was filtered through a plug of silica and eluted with EtOAc. The filtrate was concentrated under reduced pressure. Purification by column chromatography $\left(\mathrm{SiO}_{2}\right.$, petroleum ether/ethyl acetate 10/1 to $5 / 1$ ) yielded cyclopropane 17 (131 mg, $0.36 \mathrm{mmol}, 72 \%$ ) as a mixture of diastereomers, trans:cis-2.4:1.

\section{trans-17}

white oil

${ }^{1} \mathrm{H}$ NMR (400 MHz, $\left.\mathrm{CDCl}_{3}\right), \delta(\mathrm{ppm}): 7.71(\mathrm{~d}, J=8.3 \mathrm{~Hz}, 2 \mathrm{H}), 7.31(\mathrm{~d}, J=8.0 \mathrm{~Hz}, 2 \mathrm{H}), 5.78-5.68(\mathrm{~m}$, $1 \mathrm{H}), 5.21-5.28(\mathrm{~m}, 2 \mathrm{H}), 4.13(\mathrm{dq}, J=7.1,0.9 \mathrm{~Hz}, 2 \mathrm{H}), 4.02(\mathrm{~s}, 2 \mathrm{H}), 3.78(\mathrm{dd}, J=6.4,0.9 \mathrm{~Hz}, 2 \mathrm{H}$ ), $2.44(\mathrm{~s}, 3 \mathrm{H}), 1.60-1.50(\mathrm{~m}, 2 \mathrm{H}), 1.27(\mathrm{t}, J=7.1 \mathrm{~Hz}, 3 \mathrm{H}), 1.21(\mathrm{~m}, 1 \mathrm{H}), 0.80(\mathrm{~m}, 1 \mathrm{H}) ;{ }^{13} \mathrm{C}$ NMR $(100$ $\left.\mathrm{MHz}, \mathrm{CDCl}_{3}\right), \delta(\mathrm{ppm}): 172.0,143.5,136.2,132.1,129.4$ (2), 127.8 (2), 119.7, 85.5, 70.0, 61.0, 49.1, 36.1, 22.4, 21.5, 16.5, 14.2, 9.7; IR (film) $v=2981$ (w), $2923(w), 2243(w), 1725$ (s), 1644 (w), 1598 (w), $1495(\mathrm{w}), 1444(\mathrm{w}), 1407(\mathrm{~m}), 1387(\mathrm{w}), 1349(\mathrm{~m}), 1315(\mathrm{~m}), 1264(\mathrm{~m}), 1207(\mathrm{~m}), 1182(\mathrm{~m}), 1162$ (s), $1119(w), 1093(m), 1043(w), 1018(w), 993(w), 930(m), 898(m), 845(w), 816(w), 756(m), 706$ 
(w), $663(\mathrm{~m}), 578(\mathrm{~m}), 546(\mathrm{~m})$; HRMS (ESI): calcd for $\left[\mathrm{C}_{19} \mathrm{H}_{23} \mathrm{NO}_{4} \mathrm{SNa}^{+}\right]$: 384.1240; found: 384.1240; $\boldsymbol{R}_{\mathbf{f}}=0.22$ (petroleum ether/ethyl acetate 10/1).

cis-17

white oil

${ }^{1} \mathrm{H}$ NMR (400 MHz, $\left.\mathrm{CDCl}_{3}\right), \delta(\mathrm{ppm}): 7.76(\mathrm{~d}, J=8.3 \mathrm{~Hz}, 2 \mathrm{H}), 7.30(\mathrm{~d}, J=8.1 \mathrm{~Hz}, 2 \mathrm{H}), 5.76-5.66(\mathrm{~m}$, $1 \mathrm{H}), 5.32(\mathrm{dd}, J=17.0,1.4 \mathrm{~Hz}, 1 \mathrm{H}), 5.20(\mathrm{dd}, J=10.1,1.3 \mathrm{~Hz}, 1 \mathrm{H}), 4.13(\mathrm{~m}, 2 \mathrm{H}), 4.04(\mathrm{~d}, J=10.1$, $1.3 \mathrm{~Hz}, 2 \mathrm{H}), 3.80$ (d, J = 6.5 Hz, $2 \mathrm{H}), 2.43(\mathrm{~s}, 3 \mathrm{H}), 1.81(\mathrm{~m}, 1 \mathrm{H}), 1.51(\mathrm{~m}, 1 \mathrm{H}), 1.26(\mathrm{t}, J=7.1 \mathrm{~Hz}, 3$ $\mathrm{H})$, 1.14-1.04 (m, $2 \mathrm{H}) ;{ }^{13} \mathrm{C}$ NMR $\left(100 \mathrm{MHz}, \mathrm{CDCl}_{3}\right), \delta(\mathrm{ppm}): 169.9,143.2,136.3,132.1,129.4(2)$, 127.8 (2), 119.8, 83.0, 72.1, 60.7, 48.7, 36.2, 21.5, 20.8, 14.4, 14.1, 9.1; IR (film) $v=2981$ (w), 2925 (w), $2253(w), 1729(\mathrm{~s}), 1643(\mathrm{w}), 1598(\mathrm{w}), 1495(\mathrm{w}), 1445(\mathrm{~m}), 1404(\mathrm{~m}), 1382(\mathrm{~m}), 1347(\mathrm{~s}), 1306$ (w), $1183(\mathrm{~s}), 1161(\mathrm{~s}), 1119(\mathrm{~m}), 1092(\mathrm{~m}), 1059(\mathrm{~m}), 1019(\mathrm{~m}), 994(\mathrm{w}), 933(\mathrm{~m}), 894(\mathrm{~m}), 853(\mathrm{~m})$, $815(\mathrm{~m}), 757(\mathrm{~m}), 713(\mathrm{w}), 664(\mathrm{~m}), 579(\mathrm{~m}), 546(\mathrm{~m})$; HRMS (ESI): calcd for $\left[\mathrm{C}_{19} \mathrm{H}_{23} \mathrm{NO}_{4} \mathrm{SNa}^{+}\right]$: 384.1240; found: $384.1246 ; \boldsymbol{R}_{\mathfrak{f}}=0.13$ (petroleum ether/ethyl acetate $10 / 1$ ).

ethyl 2-(3-((4-methylphenyl)sulfonamido)prop-1-yn-1-yl)cyclopropane-1-carboxylate 18

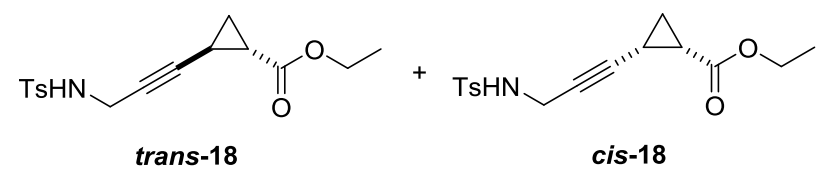

Thermal conditions:

According to GP-3, TBAFe (5.2 mg, $0.0125 \mathrm{mmol}, 2.5 \mathrm{~mol} \%)$, 4-nitroanisole (0.38 $\mathrm{mg}, 0.0025 \mathrm{mmol}$, $0.5 \mathrm{~mol} \%)$ in 1,2-DCE (250 $\mu \mathrm{L})$, 4-methyl- $N$-(pent-4-en-2-yn-1-yl)benzenesulfonamide s21 (117.7 mg, $0.5 \mathrm{mmol}, 1$ eq.), and ethyl diazoacetate (63 $\mu \mathrm{L}, 0.52 \mathrm{mmol}, 1.05$ eq.) were stirred at $40{ }^{\circ} \mathrm{C}$ for $24 \mathrm{~h}$. The reaction mixture was filtered through a plug of silica and eluted with $\mathrm{Et}_{2} \mathrm{O}$. Purification by column chromatography $\left(\mathrm{SiO}_{2}\right.$, petroleum ether/ethyl acetate $5 / 1$ to $\left.1 / 1\right)$ yielded cyclopropane 18 (30 mg, 0.10 $\mathrm{mmol}, 19 \%)$ as a mixture of diastereomers, trans:cis-1.8:1.

Photochemical conditions:

According to GP-4, TBAFe (10.3 mg, $0.025 \mathrm{mmol}, 5 \mathrm{~mol} \%)$, 1,4-dioxane (125 $\mu \mathrm{L})$, nitromethane (25 $\mu \mathrm{L}$ ), 4-methyl- $N$-(pent-4-en-2-yn-1-yl)benzenesulfonamide s21 (117.7 mg, $0.5 \mathrm{mmol}, 1$ eq.), and ethyl diazoacetate ( $63 \mu \mathrm{L}, 0.52 \mathrm{mmol}, 1.05$ eq.) were stirred at room temperature under irradiation for $20 \mathrm{~h}$. The reaction mixture was filtered through a plug of silica and eluted with EtOAc. The filtrate was concentrated under reduced pressure. Purification by column chromatography $\left(\mathrm{SiO}_{2}\right.$, petroleum ether/ethyl acetate $5 / 1$ to $1 / 1)$ yielded cyclopropane $18(63 \mathrm{mg}, 0.20 \mathrm{mmol}, 39 \%)$ as a mixture of diastereomers, trans:cis-3.2:1.

\section{trans-18}

yellow oil

${ }^{1} \mathrm{H}$ NMR $\left(300 \mathrm{MHz}, \mathrm{CDCl}_{3}\right), \delta(\mathrm{ppm}): 7.76(\mathrm{~d}, J=8.3 \mathrm{~Hz}, 2 \mathrm{H}), 7.32(\mathrm{~d}, J=8.0 \mathrm{~Hz}, 2 \mathrm{H}), 4.51$ (t, $J=5.9$ $\mathrm{Hz}, 1 \mathrm{H}$ ), 4.13 (q, J= 7.1 Hz, $2 \mathrm{H}$ ), 3.79 (dd, J=6.1, $1.4 \mathrm{~Hz}, 2 \mathrm{H}), 2.44(\mathrm{~s}, 3 \mathrm{H}), 1.60(\mathrm{~m}, 2 \mathrm{H}), 1.26$ (t, $J=7.1 \mathrm{~Hz}, 3 \mathrm{H}), 1.24(\mathrm{~m}, 1 \mathrm{H}), 0.88(\mathrm{~m}, 1 \mathrm{H}) ;{ }^{13} \mathrm{C} \mathrm{NMR}\left(100 \mathrm{MHz}, \mathrm{CDCl}_{3}\right), \delta(\mathrm{ppm}): 172.0,143.8$, 136.9, 129.6 (2), 127.5 (2), 84.8, 71.6, 61.0, 33.2, 22.4, 21.5, 16.4, 14.2, 9.7; IR (film) $v=3273$ (m), 
$2983(w), 2246(w), 1722(s), 1598(w), 1495(w), 1441(m), 1408(m), 1388(m), 1317(s), 1267(m)$, $1209(\mathrm{~m}), 1183(\mathrm{~s}), 1156(\mathrm{~s}), 1093(\mathrm{~m}), 1056(\mathrm{~m}), 1017(\mathrm{~m}), 927(\mathrm{w}), 842(\mathrm{~m}), 813(\mathrm{~m}), 770(\mathrm{w}), 708$ (m), 663 (s), $550(\mathrm{~m}), 494(\mathrm{~m})$; HRMS (ESI): calcd for $\left[\mathrm{C}_{16} \mathrm{H}_{19} \mathrm{NO}_{4} \mathrm{SNa}^{+}\right]$: 344.0927; found: 344.0927; $\boldsymbol{R}_{\mathbf{f}}=0.49$ (petroleum ether/ethyl acetate $2 / 1$ ).

\section{cis-18}

yellow oil

${ }^{1} \mathrm{H}$ NMR $\left(400 \mathrm{MHz}, \mathrm{CDCl}_{3}\right), \delta(\mathrm{ppm}): 7.75(\mathrm{~d}, J=8.3 \mathrm{~Hz}, 2 \mathrm{H}), 7.30(\mathrm{~d}, J=8.1 \mathrm{~Hz}, 2 \mathrm{H}), 4.59$ (t, $J=5.3$ $\mathrm{Hz}, 1 \mathrm{H}), 4.16(\mathrm{~m}, 2 \mathrm{H}), 3.75(\mathrm{dd}, J=5.8,1.8 \mathrm{~Hz}, 2 \mathrm{H}), 2.42(\mathrm{~s}, 3 \mathrm{H}), 1.85(\mathrm{~m}, 1 \mathrm{H}), 1.58(\mathrm{~m}, 1 \mathrm{H}), 1.26$ (t, $J=7.1 \mathrm{~Hz}, 3 \mathrm{H}), 1.22(\mathrm{~m}, 1 \mathrm{H}), 1.12(\mathrm{~m}, 1 \mathrm{H}) ;{ }^{13} \mathrm{C} \mathrm{NMR}\left(100 \mathrm{MHz}, \mathrm{CDCl}_{3}\right), \delta(\mathrm{ppm}): 170.1,143.5$, 136.7, 129.6 (2), 127.4 (2), 82.6, 73.6, 60.9, 33.5, 21.5, 20.9, 14.4, 14.3, 9.1; IR (film) $v=3265$ (m), $2981(w), 2253(w), 1716(s), 1598(w), 1561(w), 1495(w), 1434(m), 1405(m), 1382(m), 1327(m)$, $1306(\mathrm{~m}), 1184(\mathrm{~s}), 1155$ (s), $1092(\mathrm{~s}), 1054(\mathrm{~m}), 1020(\mathrm{~m}), 943(\mathrm{w}), 855(\mathrm{~m}), 813(\mathrm{~m}), 714(\mathrm{~m}), 662$ (s), $571(\mathrm{~m}), 550$ (s); HRMS (ESI): calcd for $\left[\mathrm{C}_{16} \mathrm{H}_{19} \mathrm{NO}_{4} \mathrm{SNa}^{+}\right]$: 344.0927; found: 344.0928; $\boldsymbol{R}_{\mathrm{f}}=0.34$ (petroleum ether/ethyl acetate $2 / 1$ ).

ethyl 2-(tert-butyl)-2-(4-phenylbut-1-yn-1-yl)cyclopropane-1-carboxylate 19

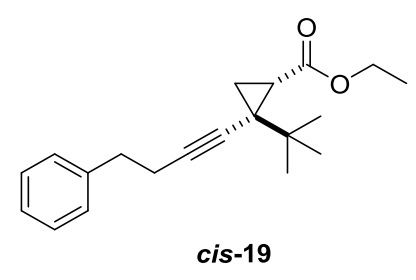

Thermal conditions:

According to GP-3, TBAFe (5.2 mg, $0.0125 \mathrm{mmol}, 2.5 \mathrm{~mol} \%)$, 4-nitroanisole (0.38 mg , $0.0025 \mathrm{mmol}$, $0.5 \mathrm{~mol} \%)$ in 1,2-DCE $(250 \mu \mathrm{L}),(6,6$-dimethyl-5-methylenehept-3-yn-1-yl)benzene s24 (106.2 mg, 0.5 mmol, 1 eq.), and ethyl diazoacetate ( $63 \mu \mathrm{L}, 0.52 \mathrm{mmol}, 1.05$ eq.) were stirred at $40^{\circ} \mathrm{C}$ for 24 hours. The reaction mixture was filtered through a plug of silica and eluted with $\mathrm{Et}_{2} \mathrm{O}$. Purification by column chromatography $\left(\mathrm{SiO}_{2}\right.$, petroleum ether/ethyl acetate $40 / 1$ to 20/1) yielded exclusively ciscyclopropane 19 (77 mg, $0.26 \mathrm{mmol}, 52 \%$ ) as a colorless oil.

Photochemical conditions:

According to GP-4, TBAFe (10.3 mg, $0.025 \mathrm{mmol}, 5 \mathrm{~mol} \%)$, 1,4-dioxane (125 $\mu \mathrm{L})$, nitromethane (25 $\mu \mathrm{L}$ ), (6,6-dimethyl-5-methylenehept-3-yn-1-yl)benzene s24 (76.6 mg, $0.5 \mathrm{mmol}, 1$ eq.), and ethyl diazoacetate ( $63 \mu \mathrm{L}, 0.52 \mathrm{mmol}, 1.05$ eq.) were stirred at room temperature under irradiation for 20 hours. The reaction mixture was filtered through a plug of silica and eluted with EtOAc. The filtrate was concentrated under reduced pressure. Purification by column chromatography $\left(\mathrm{SiO}_{2}\right.$, petroleum ether/ethyl acetate 40/1 to 10/1) yielded exclusively cis-cyclopropane 19 (78 mg, $0.26 \mathrm{mmol}, 52 \%)$ as a colorless oil.

${ }^{1} \mathrm{H}$ NMR $\left(400 \mathrm{MHz}, \mathrm{CDCl}_{3}\right), \delta(\mathrm{ppm}): 7.27(\mathrm{~m}, 2 \mathrm{H}), 7.19(\mathrm{~m}, 3 \mathrm{H}), 4.14(\mathrm{q}, J=7.1 \mathrm{~Hz}, 2 \mathrm{H}), 2.79(\mathrm{t}, J=$ $7.6 \mathrm{~Hz}, 2 \mathrm{H}), 2.44$ (t, $J=7.6 \mathrm{~Hz}, 2 \mathrm{H}), 1.86(\mathrm{~m}, 1 \mathrm{H}), 1.37(\mathrm{~m}, 1 \mathrm{H}), 1.25(\mathrm{t}, J=7.1 \mathrm{~Hz}, 3 \mathrm{H}), 1.14(\mathrm{~m}, 1$ $\mathrm{H}), 0.93(\mathrm{~s}, 9 \mathrm{H}) ;{ }^{13} \mathrm{C}$ NMR (100 MHz, $\left.\mathrm{CDCl}_{3}\right), \delta(\mathrm{ppm}): 170.9,141.0,128.5$ (2), $128.3(2), 126.1,80.1$, 
79.7, 60.5, 35.5, 33.1, 31.0, 27.0, 24.6, 20.9, 16.8, 14.4; IR (film) $v=3063$ (w), 3027 (w), $2969(\mathrm{~m})$, 2907 (w), $2868(w), 1734(s), 1603(w), 1496(w), 1454(m), 1395(m), 1379(m), 1366(m), 1251(m)$, $1182(\mathrm{~s}), 1152(\mathrm{~s}), 1097(\mathrm{~m}), 1084(\mathrm{~m}), 1064(\mathrm{w}), 1033(\mathrm{~m}), 975(\mathrm{w}), 931(\mathrm{w}), 844(\mathrm{~m}), 793(\mathrm{w}), 745$ $(\mathrm{m}), 699(\mathrm{~m}), 586(\mathrm{w}), 536(\mathrm{w}), 504(\mathrm{w})$; HRMS (ESI): calcd for $\left[\mathrm{C}_{20} \mathrm{H}_{26} \mathrm{O}_{2} \mathrm{Na}^{+}\right]$: 321.1825; found: 321.1826; $\boldsymbol{R}_{\mathfrak{f}}=0.49$ (petroleum ether/ethyl acetate $10 / 1$ ).

ethyl 2-(3-oxo-3-phenylprop-1-yn-1-yl)cyclopropane-1-carboxylate 20

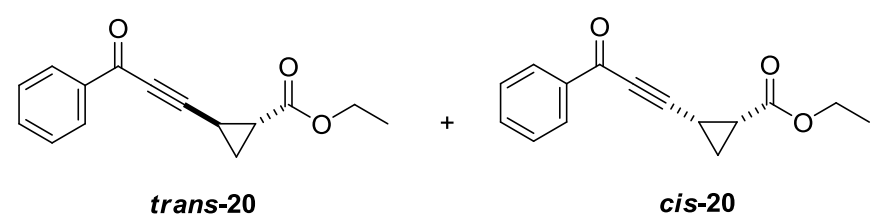

Thermal conditions:

According to GP-3, TBAFe (5.2 mg, $0.0125 \mathrm{mmol}, 2.5 \mathrm{~mol} \%)$, 4-nitroanisole $(0.38 \mathrm{mg}, 0.0025 \mathrm{mmol}$, $0.5 \mathrm{~mol} \%)$ in 1,2-DCE (250 $\mathrm{\mu L}), 1$-phenylpent-4-en-2-yn-1-one s18 (78.1 mg, $0.5 \mathrm{mmol}, 1$ eq.), and ethyl diazoacetate ( $63 \mu \mathrm{L}, 0.52 \mathrm{mmol}, 1.05$ eq.) were stirred at $40{ }^{\circ} \mathrm{C}$ for 24 hours. The reaction mixture was filtered through a plug of silica and eluted with EtOAc. Purification by column chromatography $\left(\mathrm{SiO}_{2}\right.$, petroleum ether/ethyl acetate 10/1 to 6/1) yielded cyclopropane 20 (63 mg, $0.26 \mathrm{mmol}, 52 \%$ ) as a mixture of diastereomers, trans:cis-1.0:1.

\section{Photochemical conditions:}

According to GP-4, TBAFe (10.3 mg, $0.025 \mathrm{mmol}, 5 \mathrm{~mol} \%)$, 1,4-dioxane (125 $\mu \mathrm{L})$, nitromethane (25 $\mu \mathrm{L}$ ), 1-phenylpent-4-en-2-yn-1-one $\mathbf{s} 18$ (78.1 mg, $0.5 \mathrm{mmol}, 1$ eq.), and ethyl diazoacetate (63 $\mu \mathrm{L}$, $0.52 \mathrm{mmol}, 1.05$ eq.) were stirred at room temperature under irradiation for 20 hours. The reaction mixture was filtered through a plug of silica and eluted with EtOAc. The filtrate was concentrated under reduced pressure. Purification by column chromatography $\left(\mathrm{SiO}_{2}\right.$, petroleum ether/ethyl acetate $10 / 1$ to 6/1) yielded cyclopropane 20 (66 mg, $0.27 \mathrm{mmol}, 54 \%$ ) as a mixture of diastereomers, trans:cis-1.3:1.

\section{trans-20}

yellow oil

${ }^{1} \mathrm{H}$ NMR $\left(400 \mathrm{MHz}, \mathrm{CDCl}_{3}\right), \delta(\mathrm{ppm}): 8.09$ (d, $\left.J=7.4 \mathrm{~Hz}, 2 \mathrm{H}\right), 7.60$ (t, $\left.J=7.4 \mathrm{~Hz}, 1 \mathrm{H}\right), 7.48$ (t, $J=7.7$ $\mathrm{Hz}, 2 \mathrm{H}), 4.19(\mathrm{~d}, J=7.1 \mathrm{~Hz}, 2 \mathrm{H}), 2.20(\mathrm{~m}, 1 \mathrm{H}), 2.13(\mathrm{~m}, 1 \mathrm{H}), 1.60(\mathrm{~m}, 1 \mathrm{H}), 1.44(\mathrm{~m}, 1 \mathrm{H}), 1.29(\mathrm{t}, J$ $=7.1 \mathrm{~Hz}, 3 \mathrm{H}) ;{ }^{13} \mathrm{C}$ NMR $\left(100 \mathrm{MHz}, \mathrm{CDCl}_{3}\right), \delta(\mathrm{ppm}): 177.6,171.4,136.7,134.1,129.5$ (2), $128.6(2)$, 95.7, 76.5, 61.4, 23.6, 17.3, 14.2, 10.0; IR (film) $v=3064$ (w), 2981 (w), 2937 (w), 2907 (w), 2210 (s), $1728(\mathrm{~s}), 1639(\mathrm{~s}), 1597(\mathrm{~m}), 1581(\mathrm{~m}), 1450(\mathrm{~m}), 1405(\mathrm{~m}), 1382(\mathrm{~m}), 1354(\mathrm{~m}), 1313(\mathrm{~m}), 1268(\mathrm{~s})$, $1186(\mathrm{~s}), 1141(\mathrm{w}), 1095(\mathrm{w}), 1077(\mathrm{w}), 1022(\mathrm{~m}), 946(\mathrm{~m}), 915(\mathrm{w}), 874(\mathrm{~m}), 820(\mathrm{w}), 796(\mathrm{w}), 773$ (w), 702 (s), 618 (w), 543 (w); MS (ESI): m/z (\%): 265 (100), 243 (61); HRMS (ESI): calcd for $\left[\mathrm{C}_{15} \mathrm{H}_{14} \mathrm{O}_{3} \mathrm{Na}^{+}\right]$: 265.0835; found: $265.0835 ; \boldsymbol{R}_{\mathbf{f}}=0.26$ (petroleum ether/ethyl acetate 10/1).

\section{cis-20}

yellow oil

${ }^{1} \mathbf{H}$ NMR $\left(400 \mathrm{MHz}, \mathrm{CDCl}_{3}\right), \delta(\mathrm{ppm}): 8.18(\mathrm{~m}, 2 \mathrm{H}), 7.58(\mathrm{~m}, 1 \mathrm{H}), 7.47(\mathrm{~m}, 2 \mathrm{H}), 4.23(\mathrm{dq}, J=7.1,2.6$ $\mathrm{Hz}, 2 \mathrm{H}), 2.16(\mathrm{~m}, 1 \mathrm{H}), 2.07(\mathrm{~m}, 1 \mathrm{H}), 1.70(\mathrm{~m}, 1 \mathrm{H}), 1.45(\mathrm{~m}, 1 \mathrm{H}), 1.28(\mathrm{t}, J=7.1 \mathrm{~Hz}, 3 \mathrm{H}) ;{ }^{13} \mathrm{C} \mathrm{NMR}$ 
(100 MHz, $\mathrm{CDCl}_{3}$ ), $\delta$ (ppm): 177.8, 169.7, 136.8, 133.9, 129.7 (2), 128.4 (2), 93.7, 78.5, 61.3, 22.3, 15.3, 14.3, 9.4; IR (film) $v=3065$ (w), 2982 (w), 2938 (w), 2906 (w), 2212 (s), 1726 (s), 1642 (s), 1597 $(\mathrm{m}), 1581(\mathrm{~m}), 1450(\mathrm{~m}), 1408(\mathrm{~m}), 1387(\mathrm{~m}), 1320(\mathrm{~m}), 1264(\mathrm{~s}), 1201(\mathrm{~s}), 1182(\mathrm{~s}), 1098(\mathrm{w}), 1042$ $(\mathrm{m}), 1001(\mathrm{w}), 956(\mathrm{~m}), 922(\mathrm{w}), 881(\mathrm{~m}), 842(\mathrm{w}), 824(\mathrm{w}), 796(\mathrm{w}), 743(\mathrm{w}), 701(\mathrm{~s}), 619(\mathrm{w}), 534(\mathrm{w})$, 505 (w); MS (ESI): m/z (\%): 265 (100), 243 (34); HRMS (ESI): calcd for $\left[\mathrm{C}_{15} \mathrm{H}_{14} \mathrm{O}_{3} \mathrm{Na}^{+}\right]: 265.0835$; found: $265.0832 ; \boldsymbol{R}_{\mathbf{f}}=0.13$ (petroleum ether/ethyl acetate 10/1). 


\section{Upscaling Experiments.}

\section{Synthesis of ethyl 2-((4-methoxyphenyl)ethynyl)cyclopropane-1-carboxylate 6 on $1.5 \mathrm{mmol}$ scale under thermal conditions}

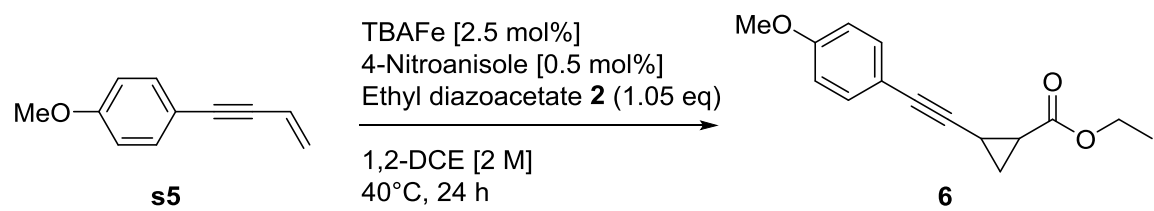

TBAFe (15.5 mg, $0.0375 \mathrm{mmol}, 2.5 \mathrm{~mol} \%)$ was loaded in a dry schlenk tube under nitrogen. A solution of 4-nitroanisole (1.15 mg , $0.0075 \mathrm{mmol}, 0.5 \mathrm{~mol} \%)$ in dry 1,2-DCE (750 $\mu \mathrm{L}), 1$-(but-3-en-1-yn-1-yl)-4methoxybenzene s5 (237.3 mg, $1.5 \mathrm{mmol}, 1$ eq.) and ethyl diazoacetate (190 $\mu \mathrm{L}, 1.57 \mathrm{mmol}, 1.05 \mathrm{eq}$.) were subsequently added and the reaction mixture was stirred at $40^{\circ} \mathrm{C}$ (heating block) for 24 hours. The reaction mixture was cooled down to room temperature, filtered through a plug of silica and eluted with $\mathrm{Et}_{2} \mathrm{O}$. The filtrate was concentrated under reduced pressure and the crude product was purified by column chromatography $\left(\mathrm{SiO}_{2}\right.$, petroleum ether/ethyl acetate 40/1 to 20/1). Cyclopropane 6 (317 $\mathrm{mg}, 1.30 \mathrm{mmol}, 86 \%$ ) was obtained as a mixture of diastereomers, trans:cis-1.9:1.

Synthesis of ethyl 2-(phenylethynyl)cyclopropane-1-carboxylate 4 on $2 \mathrm{mmol}$ scale under photochemical conditions

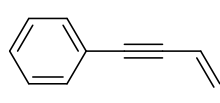

s1

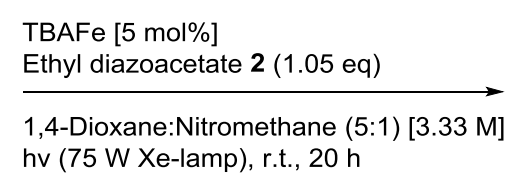

hv (75 W Xe-lamp), r.t., $20 \mathrm{~h}$

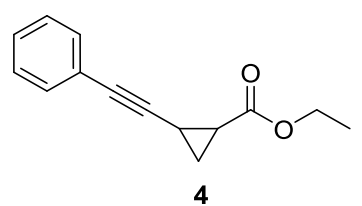

4

TBAFe (41.2 mg, $0.1 \mathrm{mmol}, 5 \mathrm{~mol} \%$ ) was loaded in a dry Schlenk tube under nitrogen. But-3-en-1-yn1-ylbenzene s1 (256.3 mg, $2 \mathrm{mmol}, 1$ eq.), 1,4-dioxane (500 $\mu \mathrm{L})$, nitromethane (100 $\mu \mathrm{L})$, and ethyl diazoacetate ( $254 \mu \mathrm{L}, 2.1 \mathrm{mmol}, 1.05$ eq.) were subsequently added. The reaction mixture was stirred at room temperature under irradiation ( $75 \mathrm{~W}$ Xe-lamp) for 20 hours, then filtered through a plug of silica and eluted with $\mathrm{Et}_{2} \mathrm{O}$. The filtrate was concentrated under reduced pressure and the crude was purified by column chromatography $\left(\mathrm{SiO}_{2}\right.$, petroleum ether/ethyl acetate $90 / 1$ to 40/1), yielding cyclopropane 4 (349 mg, $1.63 \mathrm{mmol}, 81 \%$ ) as a mixture of diastereomers, trans:cis-2.1:1. 


\section{Iron-Catalyzed Cyclopropanation of Enyne 1 in the presence of TEMPO}

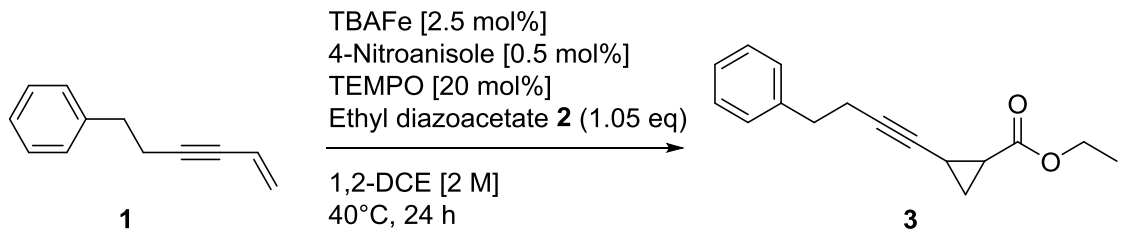

Following GP-3, TBAFe (5.2 mg, $0.0125 \mathrm{mmol}, 2.5 \mathrm{~mol} \%)$, 4-nitroanisole (0.38 mg , $0.0025 \mathrm{mmol}, 0.5$ mol\%) in 1,2-DCE (250 $\mu \mathrm{L})$, hex-5-en-3-yn-1-ylbenzene 1 (78.1 mg, $0.5 \mathrm{mmol}, 1$ eq.), ethyl diazoacetate (63 $\mu \mathrm{L}, 0.52 \mathrm{mmol}, 1.05$ eq.), and TEMPO (15.6 mg, $0.10 \mathrm{mmol}, 20 \mathrm{~mol} \%$ ) were stirred at $40{ }^{\circ} \mathrm{C}$ (heating block) for 24 hours. The reaction mixture was filtered through a plug of silica and eluted with $\mathrm{Et}_{2} \mathrm{O}$. After concentration under reduced pressure, cyclopropane $\mathbf{3}$ was obtained as a mixture of diastereomers in $45 \%$ yield (yield determined by ${ }^{1} \mathrm{H}$ NMR integration, using mesitylene as internal standard).
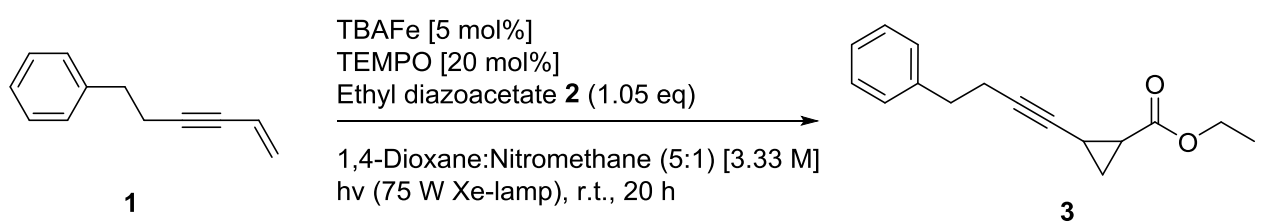

According to GP-4, TBAFe (10.3 mg, $0.025 \mathrm{mmol}, 5 \mathrm{~mol} \%)$, 1,4-dioxane (125 $\mu \mathrm{L})$, nitromethane $(25$ $\mu \mathrm{L}$ ), hex-5-en-3-yn-1-ylbenzene 1 (78.1 mg, $0.5 \mathrm{mmol}, 1$ eq.), ethyl diazoacetate ( $63 \mu \mathrm{L}, 0.52 \mathrm{mmol}$, 1.05 eq.), and TEMPO (15.6 mg, $0.10 \mathrm{mmol}, 20 \mathrm{~mol} \%$ ) were stirred at room temperature under irradiation for 20 hours. The reaction mixture was filtered through a plug of silica and eluted with $\mathrm{Et}_{2} \mathrm{O}$. After concentration under reduced pressure, cyclopropane $\mathbf{3}$ was obtained as a mixture of diastereomers in $37 \%$ yield (yield determined by ${ }^{1} \mathrm{H}$ NMR integration, using mesitylene as internal standard). 


\section{Product transformation}

\section{(2-(Phenylethynyl)cyclopropyl)methanol $21^{[27]}$}

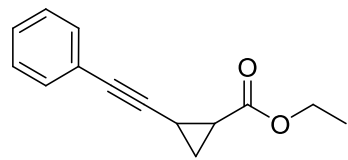

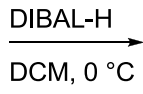

$\mathrm{CM}, 0{ }^{\circ} \mathrm{C}$

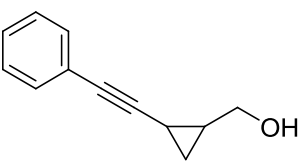

21

To a solution of ethyl 2-(phenylethynyl)cyclopropane-1-carboxylate 4 ( $86 \mathrm{mg}, 0.4 \mathrm{mmol}, 1$ eq.) in dry DCM (0.3 mL) was added DIBAL-H (1 M in heptane, $0.92 \mathrm{~mL}, 0.92 \mathrm{mmol}, 2.3$ eq.), dropwise, at $0{ }^{\circ} \mathrm{C}$. After stirring for 1.5 hours at $0{ }^{\circ} \mathrm{C}$, a saturated solution of potassium sodium tartrate was added to quench the reaction. The mixture was extracted four times with $\mathrm{DCM}$, the organic layers were combined, washed with brine, dried over anhydrous $\mathrm{Na}_{2} \mathrm{SO}_{4}$, filtered and concentrated under reduced pressure, yielding alcohol 21 (61 mg, $0.35 \mathrm{mmol}, 88 \%$ ) as a mixture of diastereomers.

\section{trans-21}

yellow oil

${ }^{1}$ H NMR $\left(400 \mathrm{MHz}, \mathrm{CDCl}_{3}\right), \delta(\mathrm{ppm}): 7.38(\mathrm{~m}, 2 \mathrm{H}), 7.27(\mathrm{~m}, 3 \mathrm{H}), 3.57(\mathrm{~m}, 2 \mathrm{H}), 1.62(\mathrm{brs}, 1 \mathrm{H}), 1.57$ $(\mathrm{m}, 1 \mathrm{H}), 1.41(\mathrm{~m}, 1 \mathrm{H}), 1.04(\mathrm{~m}, 1 \mathrm{H}), 0.86(\mathrm{~m}, 1 \mathrm{H}) ;{ }^{13} \mathrm{C}$ NMR $\left(100 \mathrm{MHz}, \mathrm{CDCl}_{3}\right), \delta(\mathrm{ppm}):$ 131.6, 128.2 (2), 127.6 (2), 123.7, 91.8, 65.5, 24.5, 13.3, 5.7; IR (film) $v=3328$ (m), 3080 (w), 2924 (w), 2874 (w), $2227(w), 1597(w), 1491(m), 1442(w), 1410(w), 1315(w), 1263(w), 1239(w), 1149(w), 1096$ (w), 1070 (w), 1043 (s), 1025 (s), 967 (m), 913 (w), 875 (w), 839 (w), 755 (s), 691 (s), 541 (w), $492(w)$; HRMS (El): calcd for $\left[\mathrm{C}_{12} \mathrm{H}_{12} \mathrm{O}\right]: 172.0888$ found: $172.0886 ; \boldsymbol{R}_{\mathbf{f}}=0.33$ (petroleum ether/ethyl acetate 2/1).

cis-21

yellow oil

${ }^{1}$ H NMR $\left(400 \mathrm{MHz}, \mathrm{CDCl}_{3}\right), \delta(\mathrm{ppm}): 7.38(\mathrm{~m}, 2 \mathrm{H}), 7.28(\mathrm{~m}, 3 \mathrm{H}), 3.96(\mathrm{dd}, J=11.8,5.4 \mathrm{~Hz}, 1 \mathrm{H})$, 3.70 (dd, $J=11.8,8.7 \mathrm{~Hz}, 1 \mathrm{H}), 1.88$ (brs, $1 \mathrm{H}), 1.70(\mathrm{~m}, 1 \mathrm{H}), 1.50(\mathrm{~m}, 1 \mathrm{H}), 1.45(\mathrm{~m}, 1 \mathrm{H}), 1.11(\mathrm{~m}, 1$ $\mathrm{H}), 0.74(\mathrm{~m}, 1 \mathrm{H}) ;{ }^{13} \mathrm{C}$ NMR (100 MHz, $\left.\mathrm{CDCl}_{3}\right), \delta(\mathrm{ppm}): 131.6,128.3(2), 127.8$ (2), 123.4, 89.6, 78.3, 64.1, 21.1, 13.2, 5.2; IR (film) $v=3334$ (m), 3079 (w), 3017 (w), 2928 (w), 2877 (w), 2223 (w), 1597 (w), $1571(w), 1490(m), 1442(w), 1402(w), 1357(w), 1330(w), 1242(w), 1143(w), 1095(w), 1069$ (w), 1043 (s), 1017 (s), 976 (m), 915 (w), 820 (w), 754 (s), 690 (s), 592 (w), 537 (w), 524 (w), 451 (w); HRMS (El): calcd for $\left[\mathrm{C}_{12} \mathrm{H}_{12} \mathrm{O}\right]: 172.0888$; found: $172.0885 ; \boldsymbol{R}_{\mathfrak{f}}=0.31$ (petroleum ether/ethyl acetate 2/1). 
Ethyl 2-(3-Phenyl-1H-pyrazol-5-yl)cyclopropane-1-carboxylate $22^{[28]}$

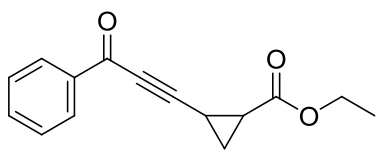

20
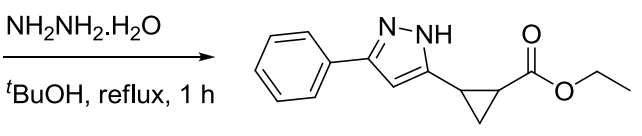

22

To a solution of ethyl 2-(3-oxo-3-phenylprop-1-yn-1-yl)cyclopropane-1-carboxylate 20 (48 mg, 0.2 mmol, 1 eq.) in ${ }^{t} \mathrm{BuOH}(0.3 \mathrm{~mL})$ was added hydrazine hydrate $(19 \mu \mathrm{L}, 0.4 \mathrm{mmol}, 2$ eq.) at room temperature. The resulting mixture was refluxed for 1 hour, then quenched by addition a saturated solution of $\mathrm{NH}_{4} \mathrm{Cl}$. The mixture was extracted three times with $\mathrm{DCM}$, the organic layers were combined, washed with brine, dried over anhydrous $\mathrm{Na}_{2} \mathrm{SO}_{4}$, filtered and concentrated under reduced pressure. Purification by column chromatography $\left(\mathrm{SiO}_{2}\right.$, petroleum ether/ethyl acetate $1 / 1$ to $\left.1 / 3\right)$ yielded pyrazole 22 (42 mg, $0.16 \mathrm{mmol}, 82 \%$ ) as a mixture of diastereomers.

\section{trans-22}

yellow oil

${ }^{1} \mathrm{H}$ NMR $\left(400 \mathrm{MHz}, \mathrm{CDCl}_{3}\right), \delta(\mathrm{ppm}): 8.80$ (brs, $\left.1 \mathrm{H}\right), 7.73(\mathrm{~d}, J=7.4 \mathrm{~Hz}, 2 \mathrm{H}), 7.38(\mathrm{t}, J=7.6 \mathrm{~Hz}, 2 \mathrm{H})$, $7.30(\mathrm{~m}, 1 \mathrm{H}), 6.50(\mathrm{~s}, 1 \mathrm{H}), 4.06(\mathrm{~m}, 2 \mathrm{H}), 2.50(\mathrm{~m}, 1 \mathrm{H}), 2.13(\mathrm{~m}, 1 \mathrm{H}), 1.68(\mathrm{~m}, 1 \mathrm{H}), 1.49(\mathrm{~m}, 1 \mathrm{H})$, $1.14(\mathrm{t}, J=7.1 \mathrm{~Hz}, 3 \mathrm{H}) ;{ }^{13} \mathrm{C}$ NMR $\left(100 \mathrm{MHz}, \mathrm{CDCl}_{3}\right), \delta(\mathrm{ppm}): 171.7,149.4,143.6,132.4,128.7(2)$, 127.8 (2), 125.6, 103.1, 61.0, 21.9, 17.5, 14.1, 13.3; IR (film) $v=3202(\mathrm{~m}), 2979$ (m), 2930 (w), 1718 (s), 1572 (w), 1504 (w), 1463 (w), 1403 (w), 1381 (m), 1356 (w), 1269 (w), 1180 (s), 1095 (m), 1073 $(\mathrm{m}), 1030(\mathrm{~m}), 1007(\mathrm{~m}), 965(\mathrm{w}), 955(\mathrm{w}), 913(\mathrm{w}), 852(\mathrm{w}), 812(\mathrm{~m}), 763(\mathrm{~s}), 731(\mathrm{w}), 692(\mathrm{~s}), 511$ (w); HRMS (ESI): calcd for $\left[\mathrm{C}_{15} \mathrm{H}_{16} \mathrm{~N}_{2} \mathrm{O}_{2} \mathrm{Na}^{+}\right]$: 279.1104, found: 279.1105; $\boldsymbol{R}_{\boldsymbol{f}}=0.50$ (petroleum ether/ethyl acetate 1/1).

\section{cis-22}

yellow oil

${ }^{1} \mathrm{H}$ NMR $\left(400 \mathrm{MHz}, \mathrm{CDCl}_{3}\right), \delta(\mathrm{ppm}): 9.37$ (brs, $\left.1 \mathrm{H}\right), 7.60(\mathrm{~d}, J=7.4 \mathrm{~Hz}, 2 \mathrm{H}), 7.38-7.29(\mathrm{~m}, 3 \mathrm{H}), 6.27$ (s, 1H), $4.16(\mathrm{t}, J=7.1 \mathrm{~Hz}, 2 \mathrm{H}), 2.54(\mathrm{~m}, 1 \mathrm{H}), 2.01(\mathrm{~m}, 1 \mathrm{H}), 1.56(\mathrm{~m}, 1 \mathrm{H}), 1.36(\mathrm{~m}, 1 \mathrm{H}), 1.27(\mathrm{t}, J=$ $7.1 \mathrm{~Hz}, 3 \mathrm{H}) ;{ }^{13} \mathrm{C}$ NMR $\left(100 \mathrm{MHz}, \mathrm{CDCl}_{3}\right), \delta(\mathrm{ppm}): 173.0,149.4,147.4,130.8,128.9$ (2), 128.3 (2), 125.6, 100.2, 60.9, 23.1, 18.7, 16.1, 14.2; IR (film) $v=3135$ (m), 2978 (m), 2923 (m), 2853 (w), 1720 (s), $1572(w), 1506(w), 1464(w), 1448(m), 1400(m), 1380(m), 1304(w), 1267(m), 1180(s), 1111$ (w), 1075 (w), 1046 (w), 1003 (w), 965 (w), 928 (w), 849 (m), 804 (w), 761 (s), 692 (s), 513 (w); HRMS (ESI): calcd for $\left[\mathrm{C}_{15} \mathrm{H}_{16} \mathrm{~N}_{2} \mathrm{O}_{2} \mathrm{Na}^{+}\right]$: 279.1104 ; found: $279.1105 ; \boldsymbol{R}_{\mathfrak{f}}=0.33$ (petroleum ether/ethyl acetate $1 / 1)$. 


\section{Comparative experiments}

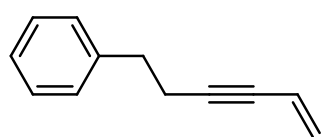

1

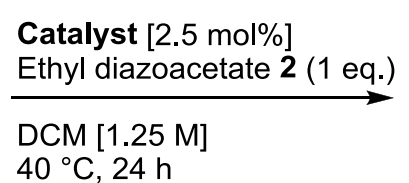

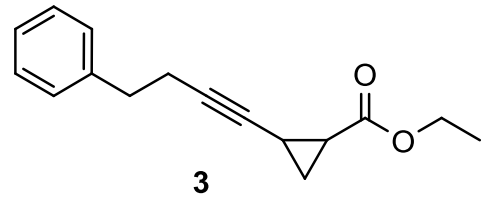

3

\section{General procedure 5 (GP-5): Cyclopropanation of enynes using different cataysts.}

The corresponding catalyst $(0.0125 \mathrm{mmol}, 2.5 \mathrm{~mol} \%)$ was loaded in a dry schlenk tube under nitrogen, then hex-5-en-3-yn-1-ylbenzene 1 (78.1 mg, $0.5 \mathrm{mmol}, 1$ eq.) and dry DCM $(250 \mu \mathrm{L})$ were added. $\mathrm{A}$ solution of ethyl diazoacetate 2 ( $66 \mu \mathrm{L}, 0.5 \mathrm{mmol}, 1$ eq.) in dry DCM $(150 \mu \mathrm{L})$ was added over a period of 30 minutes to 1 hour at room temperature. The reaction mixture was heated up to $40^{\circ} \mathrm{C}$ (heating block) and stirred for 24 hours. The reaction mixture was filtered through a plug of silica and eluted with $\mathrm{Et}_{2} \mathrm{O}$. The filtrate was concentrated under reduced pressure and the crude cyclopropane $\mathbf{3}$ was purified by column chromatography.

Table S9: Comparison with other catalysts.

\begin{tabular}{llll} 
Entry $^{[\mathrm{ac}]}$ & Catalyst & Trans:cis $^{[\mathrm{b}]}$ & Yield $^{[\mathrm{c}]}$ \\
\hline $\mathbf{1}$ & $\mathrm{Rh}_{2}(\mathrm{OAc})_{2}$ & $1.6: 1$ & $22 \%$ \\
$\mathbf{2}$ & $\mathrm{Fe}(\mathrm{TPP}) \mathrm{Cl}$ & $2.7: 1$ & $20 \%$ \\
$\mathbf{3}$ & $\mathrm{AgOTf}$ & $6 \%$ \\
$\mathbf{4}$ & $\mathrm{CuOTf}$ & $2.1: 1$ & $11 \%$ \\
$\mathbf{5}$ & $\mathrm{Cu}(\mathrm{acac})_{2}$ & $2.3: 1$ & $14 \%$ \\
$\mathbf{6}$ & $\mathrm{AuCl}$ & $3.8: 1$ & $11 \%$ \\
$\mathbf{7}$ & $\mathrm{AuCl}_{3}$ & $2.1: 1$ & traces \\
$\mathbf{8}$ & $\mathrm{Cp}^{*} \mathrm{RuCl}(\mathrm{COD})$ & - & traces \\
\hline
\end{tabular}

${ }^{[a]}$ All reactions were performed on $0.5 \mathrm{mmol}$ scale in dry DCM at $40^{\circ} \mathrm{C}$ under a nitrogen atmosphere for $24 \mathrm{~h} ;{ }^{[b]}$ trans/cis-ratio determined by GC-analysis on the crude mixture; ${ }^{[c]}$ Isolated yield; ${ }^{[\mathrm{dd}}$ Shielded from light. 


\section{References}

${ }^{[1]}$ Angew. Chem. Int. Ed. 2009, 48, 7251-7255

${ }^{[2]}$ Org. Lett. 2016, 18, 1756-1759

${ }^{[3]}$ Chem. Eur. J. 2014, 20, 8599-8602

${ }^{[4]}$ Eur. J. Org. Chem. 2009, 17, 2836-2844

${ }^{[5]}$ Angew. Chem. Int. Ed. 2015, 54, 1666-1670

${ }^{\left[{ }^{[6]}\right.}$ Tetrahedron, 2005, 61, 4043-4052

${ }^{[7]} \mathrm{J}$. Org. Chem. 2015, 80, 7311-7316

${ }^{[8]} \mathrm{J}$. Am. Chem. Soc. 2015, 137, 42-45

${ }^{[9]}$ Chem. Eur. J. 2018, 24, 3725-3728

${ }^{[10]}$ Eur. J. Org. Chem. 2006, 17, 4044-4054

${ }^{[11]} \mathrm{J}$. Am. Chem. Soc. 2014, 136, 11304-11307

${ }^{[12]}$ Chem. Commun. 2014, 50, 7666-7669

${ }^{[13]}$ Chem. Commun. 2014, 50, 10699-10702

${ }^{[14]}$ Eur. J. Med. Chem. 2016, 122, 436-441

${ }^{[15]}$ Org. Lett. 2019, 21, 535-539

${ }^{[16]}$ Adv. Synth. Catal. 2012, 354, 1149-1155

${ }^{[17]} \mathrm{J}$. Am. Chem. Soc. 2019, 141, 548-559

${ }^{[18]}$ Chem. Commun. 2012, 48, 3617-3619

${ }^{[19]}$ Org. Lett. 2018, 20, 1038-1041

${ }^{[20]}$ Org. Lett. 2004, 6, 2035-2038

${ }^{[21]} \mathrm{J}$. Am. Chem. Soc. 2004, 126, 4130-4131

${ }^{[22]}$ Chem. Eur. J. 2012, 18, 16823-16827

${ }^{[23]}$ Chem. Commun. 2017, 53, 2158-2161

${ }^{[24]} \mathrm{J}$. Org. Chem. 2000, 65, 4131-4137

${ }^{[25]} \mathrm{J}$. Am. Chem. Soc. 2012, 134, 16551-16553

${ }^{[26]}$ Chem. Ber. 1995, 128, 987-990

${ }^{[27]}$ Eur. J. Org. Chem. 2013, 23, 5165-5170

${ }^{[28]}$ J. Org. Chem. 2014, 79, 2049-2058 
10. ${ }^{1} \mathrm{H}$ and ${ }^{13} \mathrm{C}$ NMR Spectra

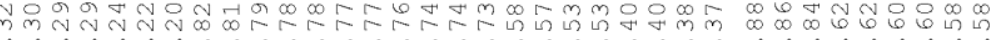

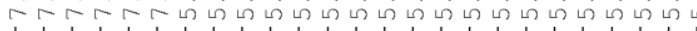

$\longrightarrow$

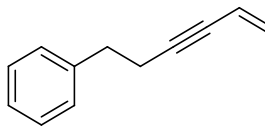

1
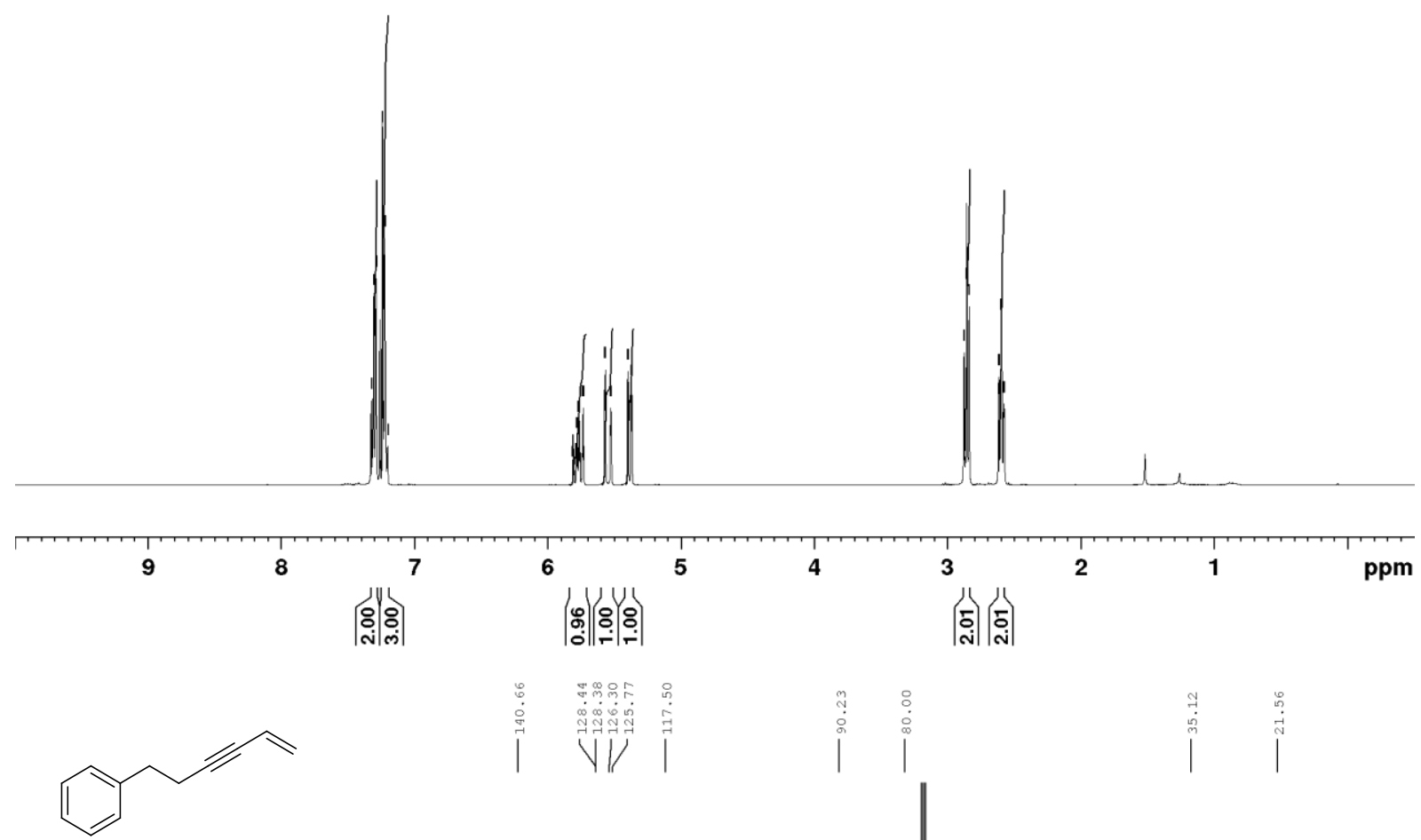

80 

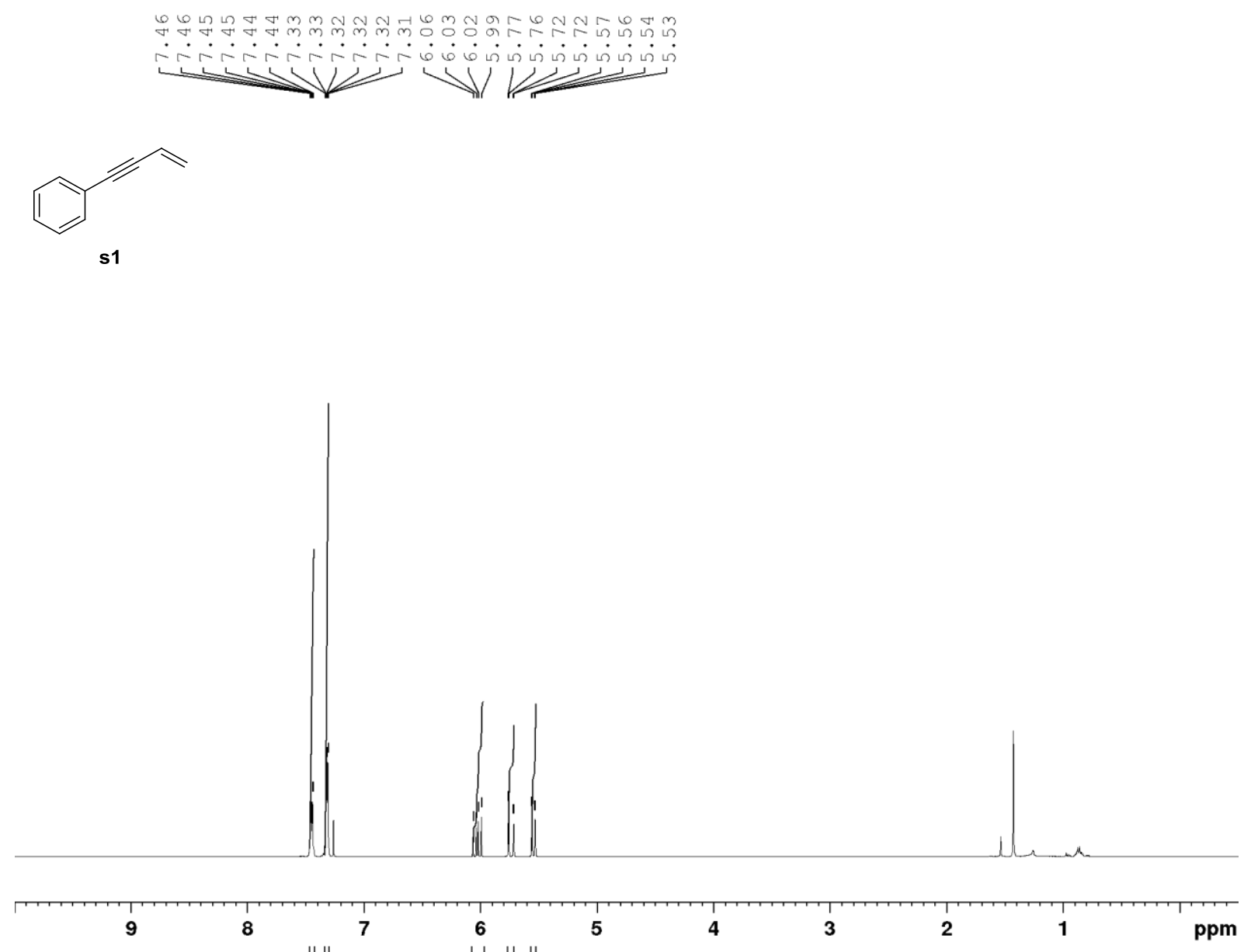

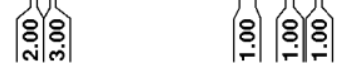

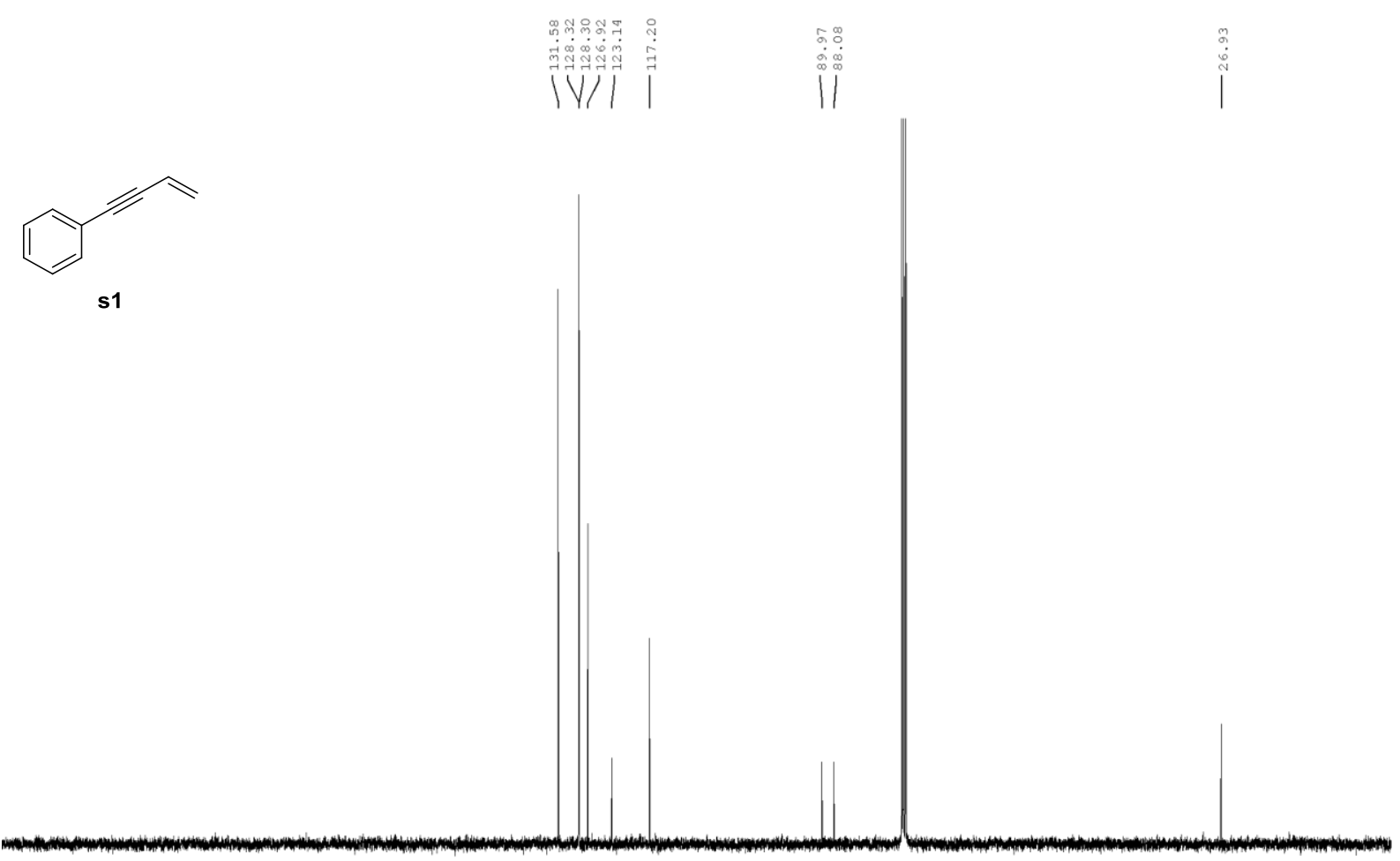

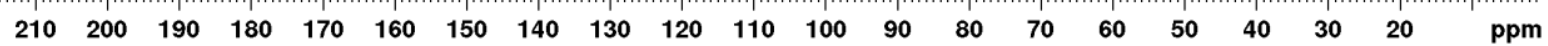



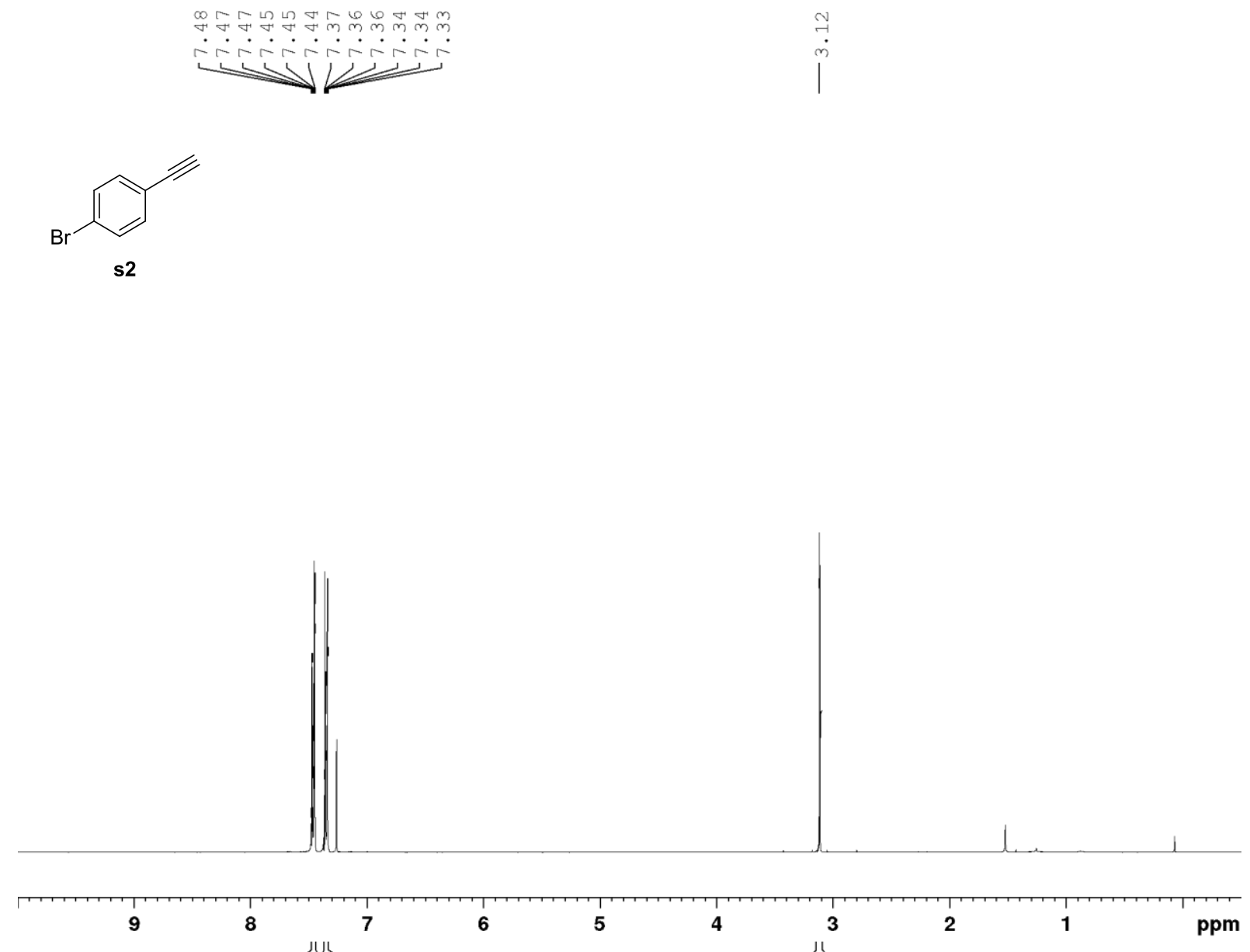

해음

움ํ.

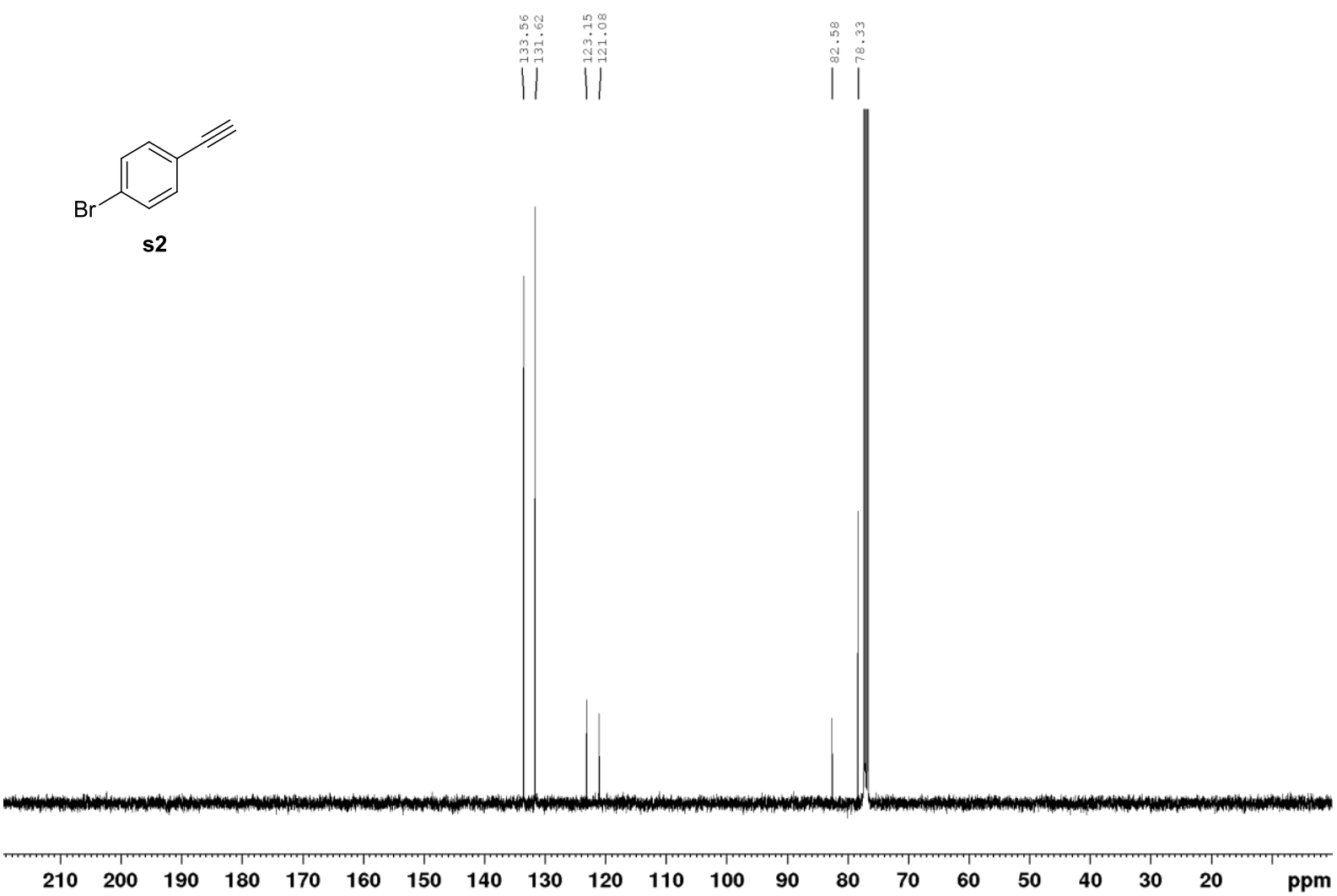



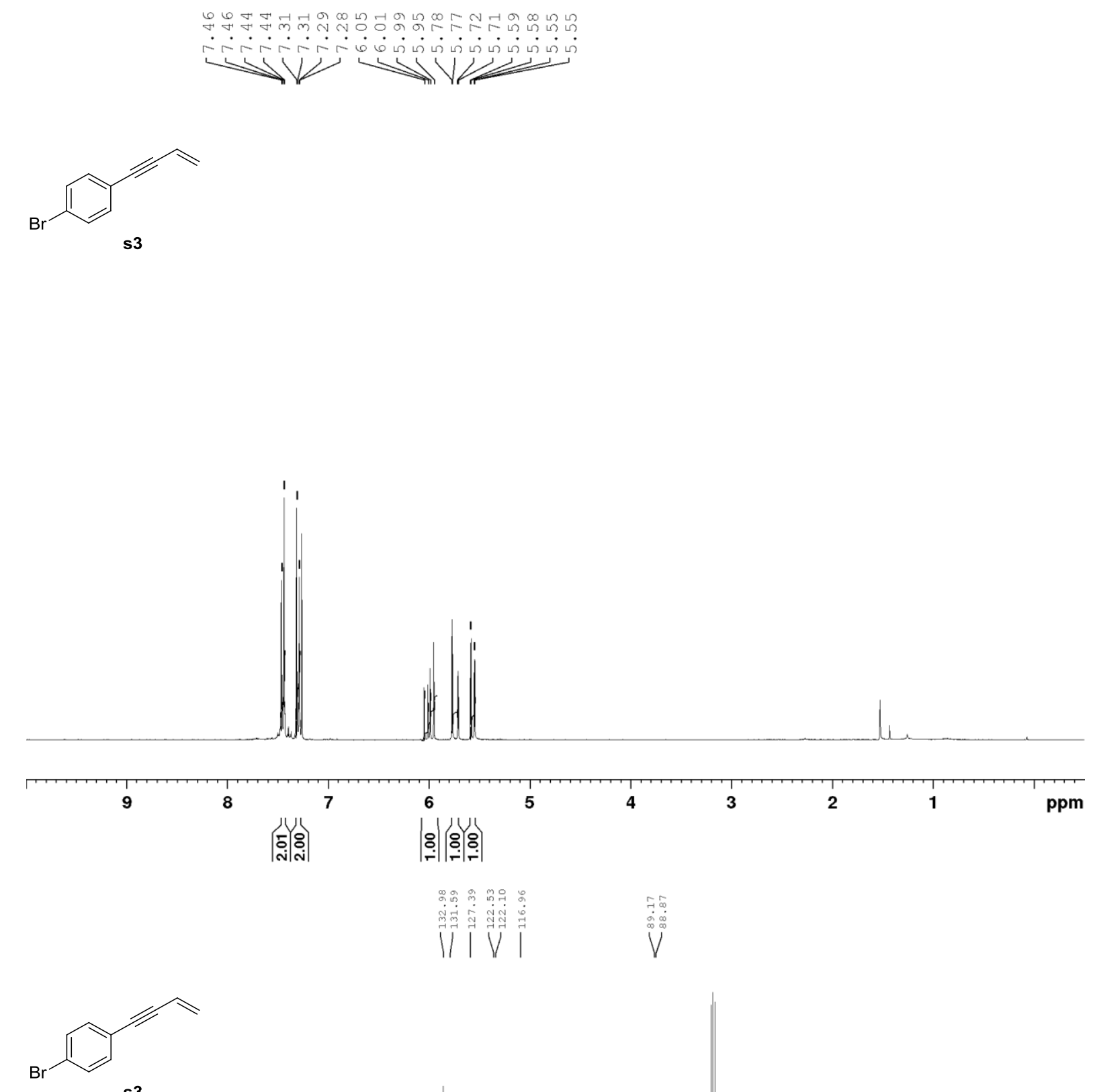

s3

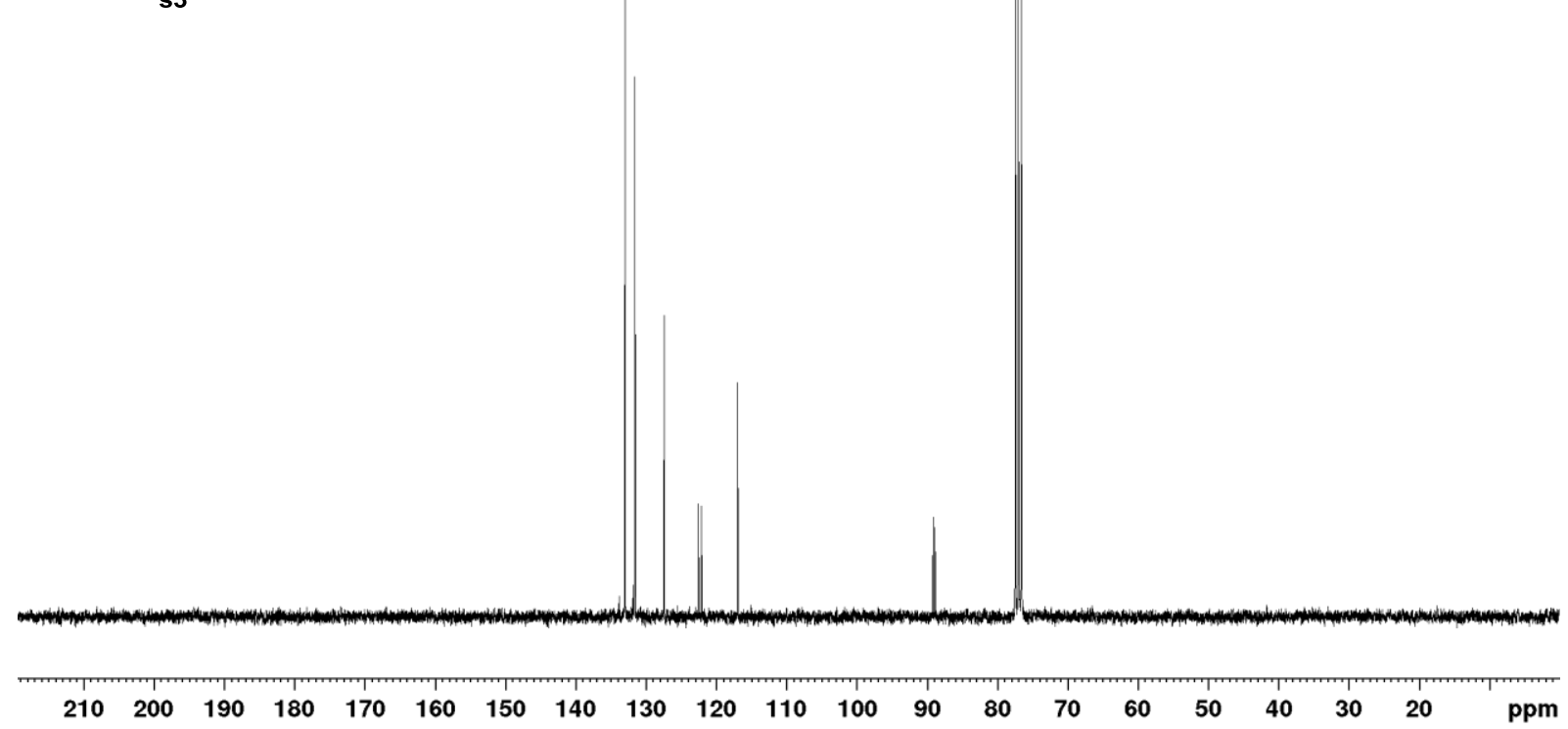




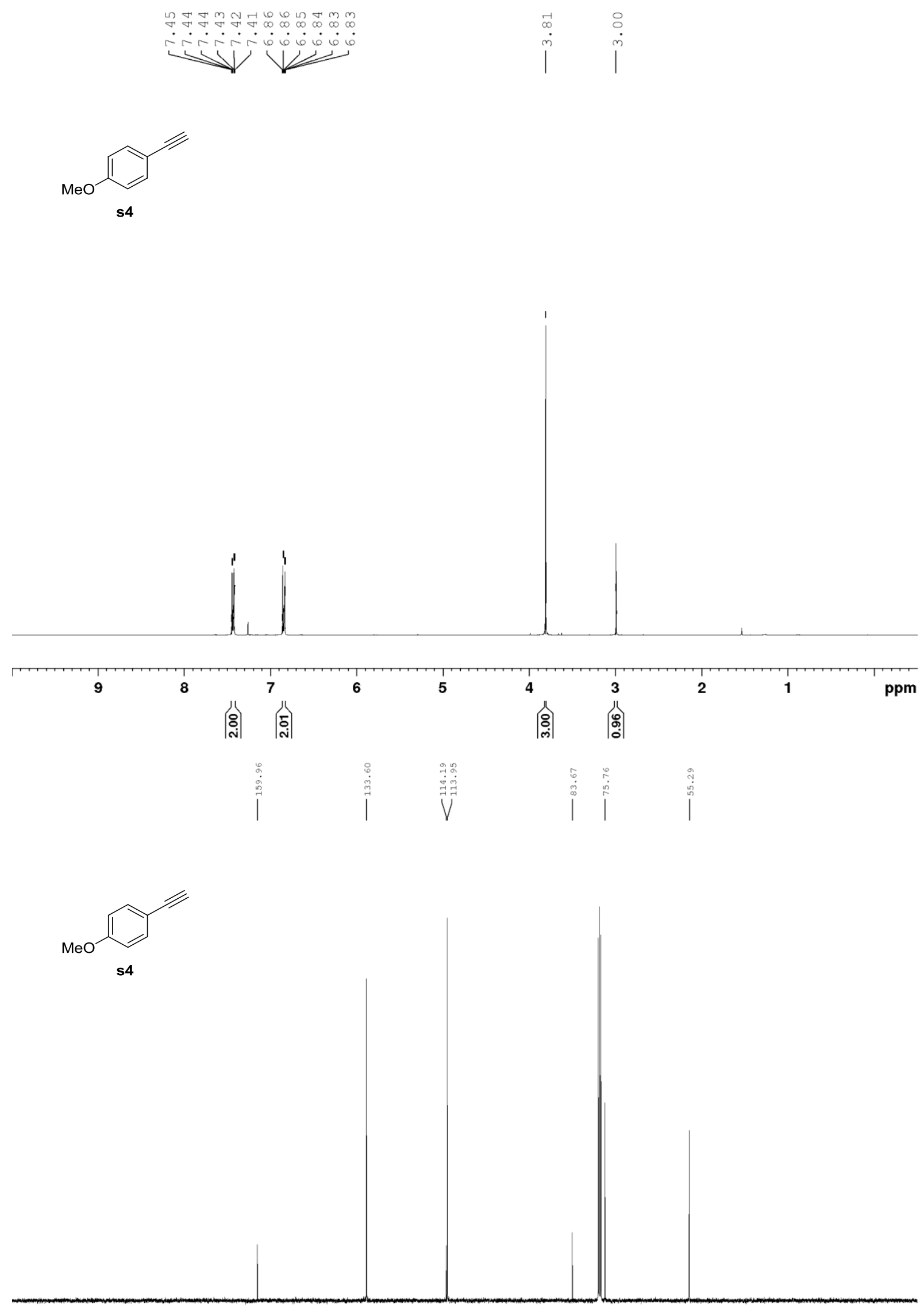

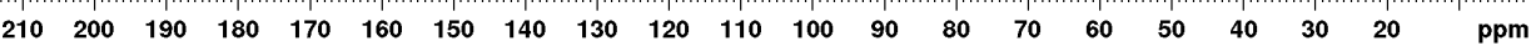



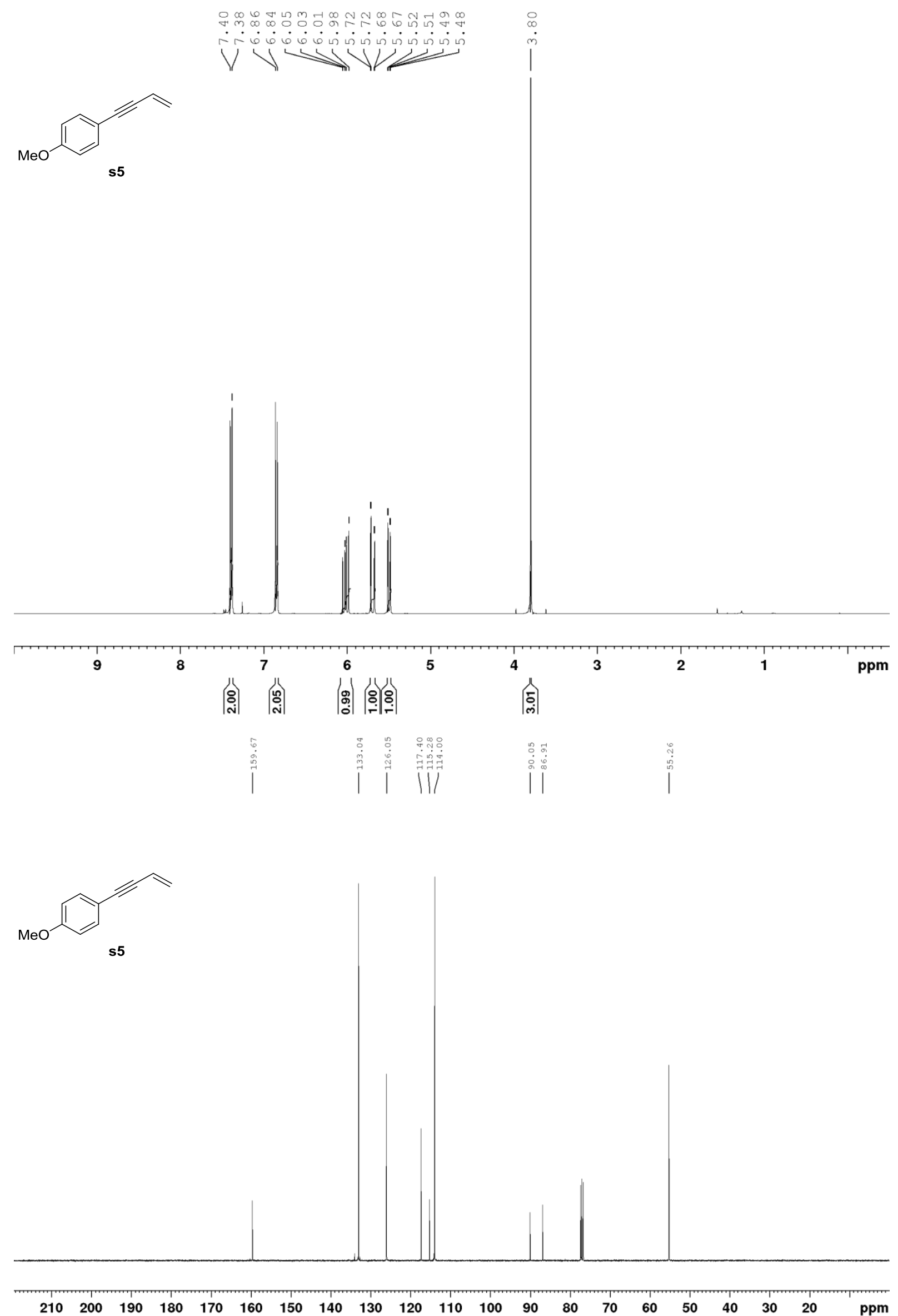

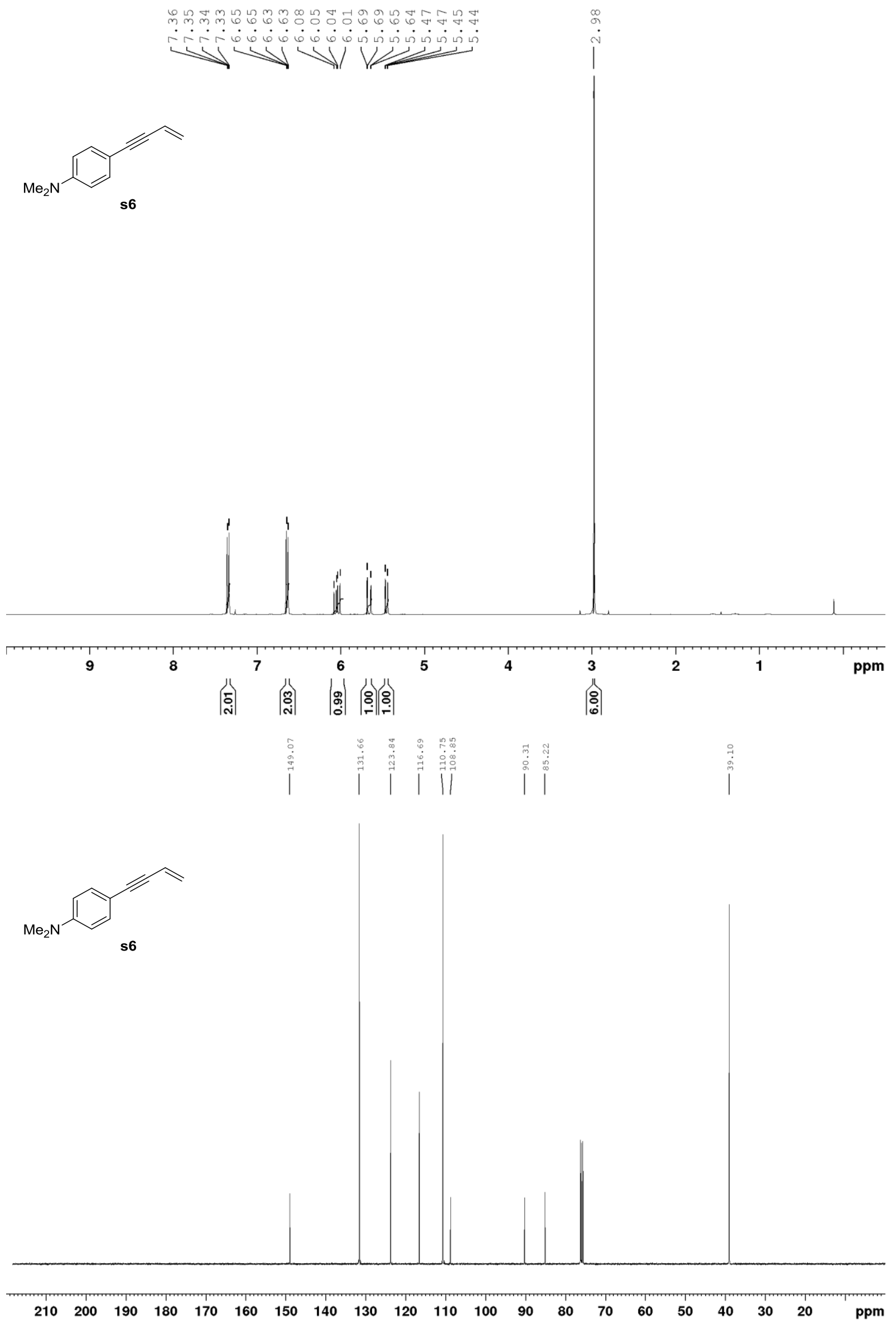

S54 


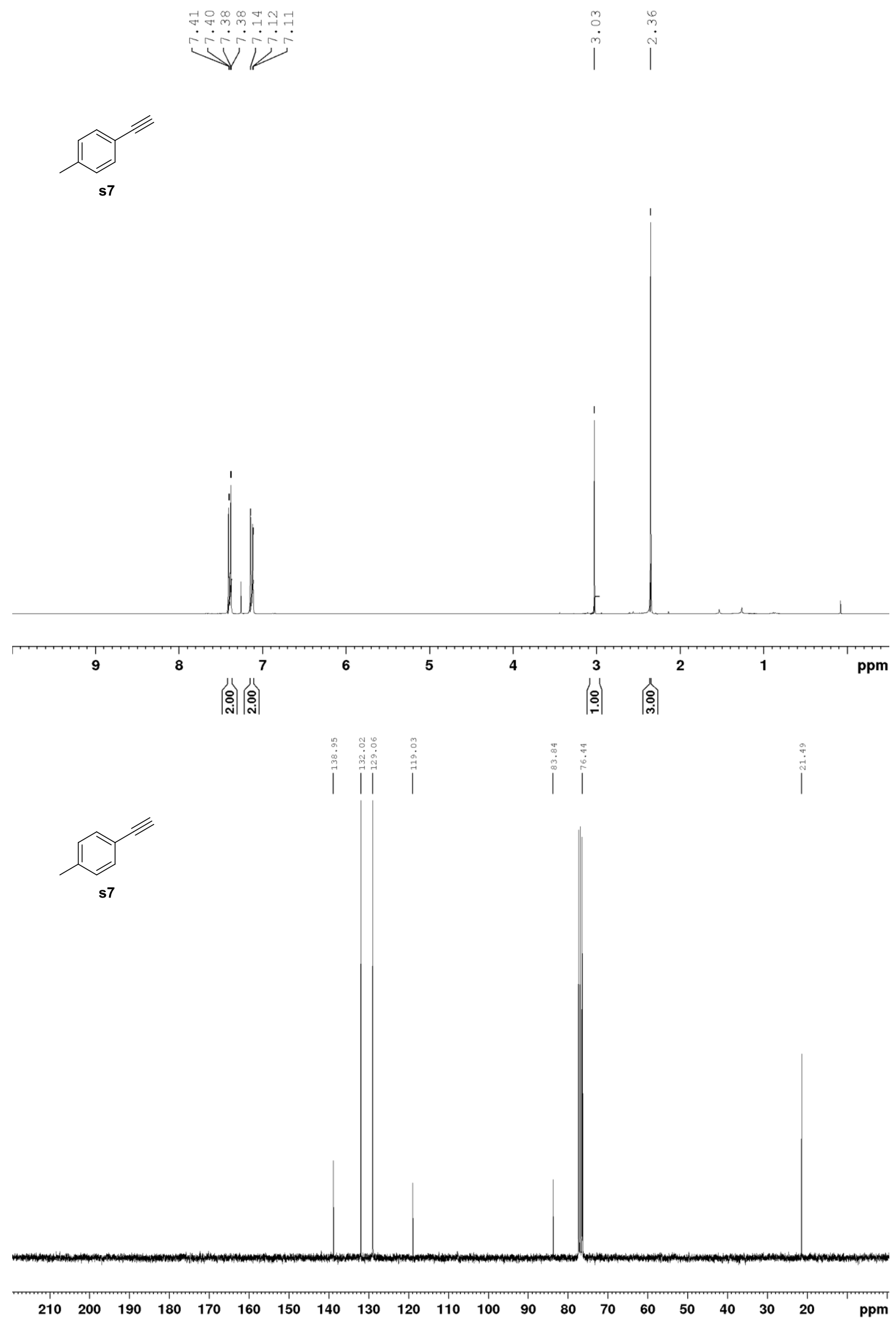




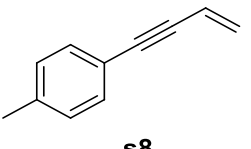

58

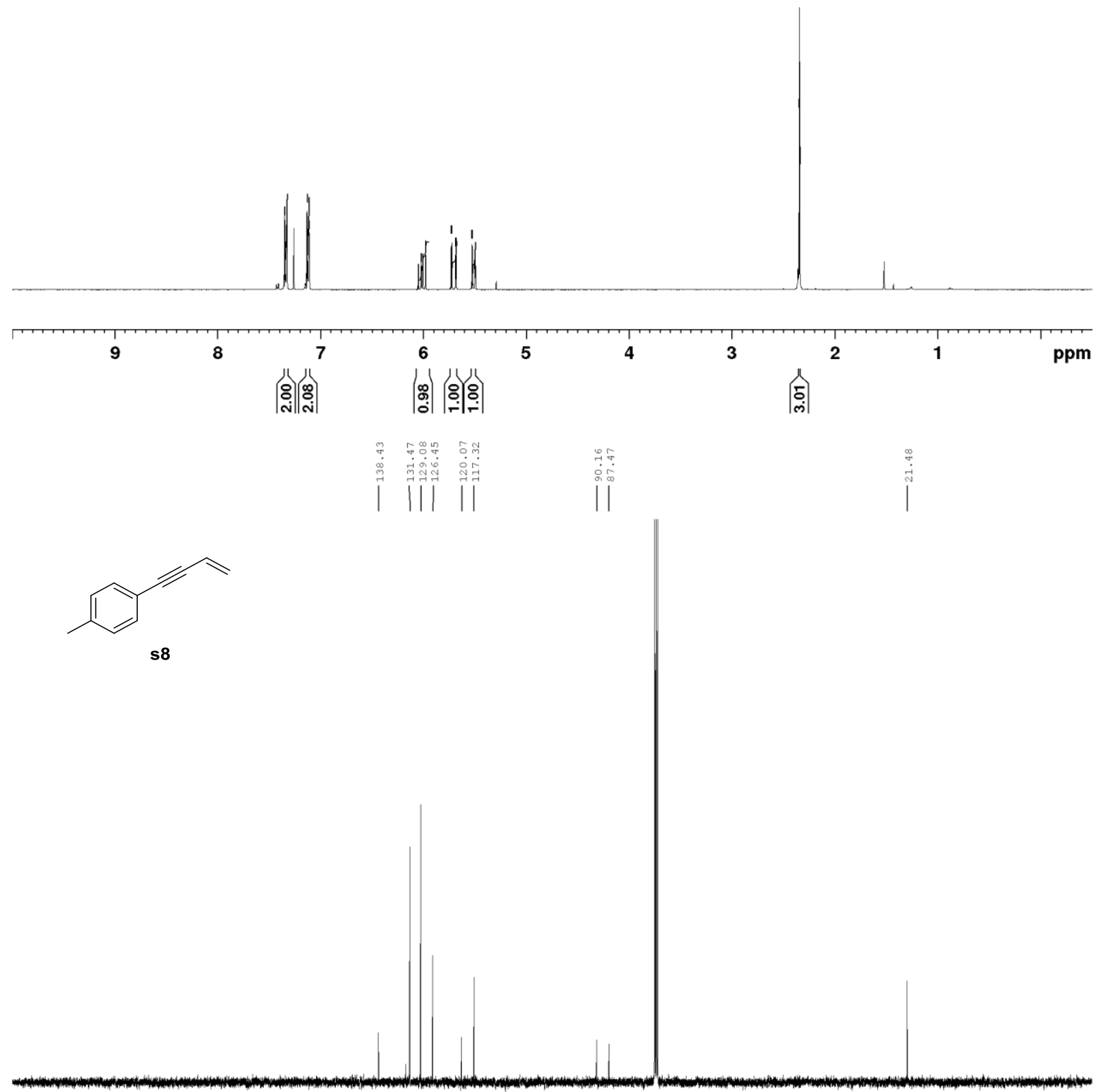

$\begin{array}{lllllllllllllllllllllll}210 & 200 & 190 & 180 & 170 & 160 & 150 & 140 & 130 & 120 & 110 & 100 & 90 & 80 & 70 & 60 & 50 & 40 & 30 & 20 & 10 & 0 & \mathrm{ppm}\end{array}$ 


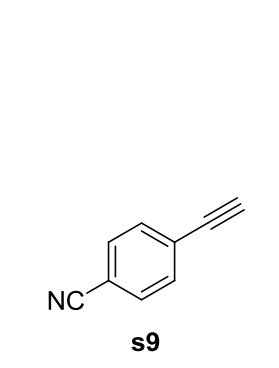

$\underbrace{2}$
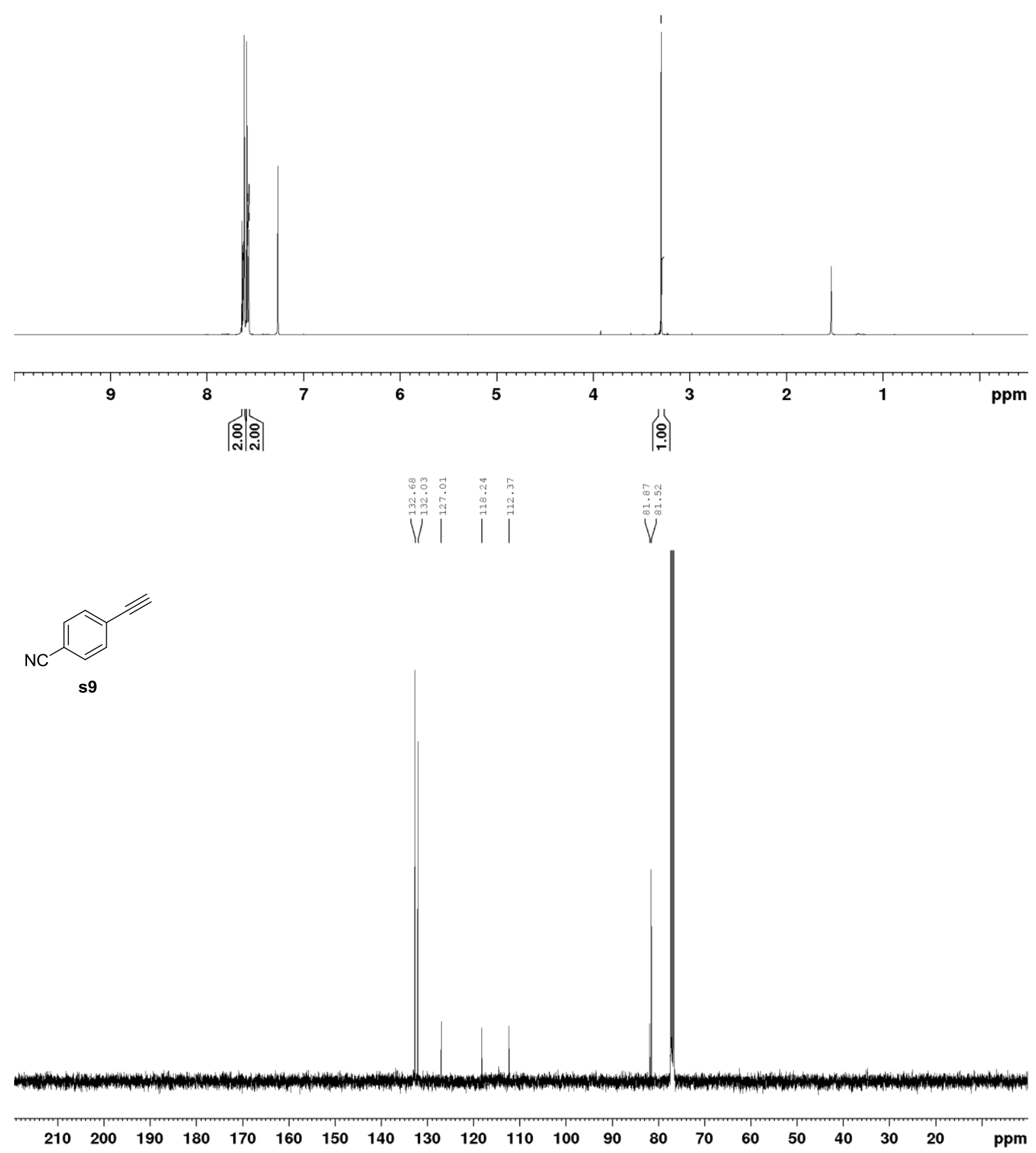

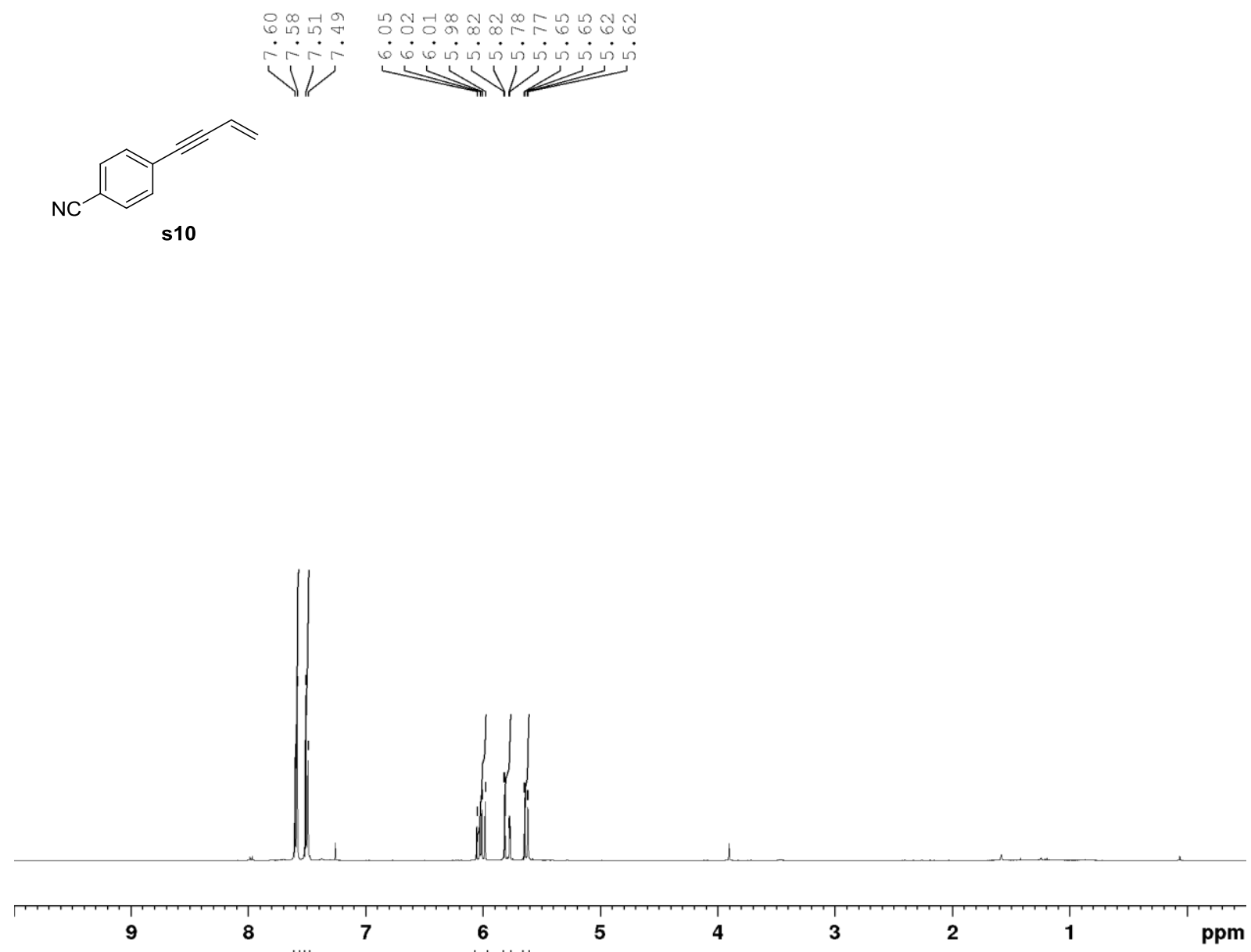

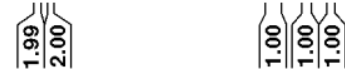
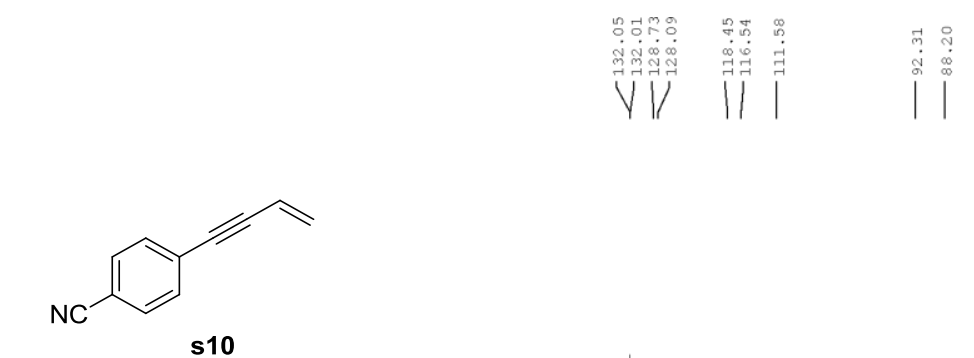

$\begin{array}{llllllllllllllllllllll}210 & 200 & 190 & 180 & 170 & 160 & 150 & 140 & 130 & 120 & 110 & 100 & 90 & 80 & 70 & 60 & 50 & 40 & 30 & 20 & \mathrm{ppm}\end{array}$ 

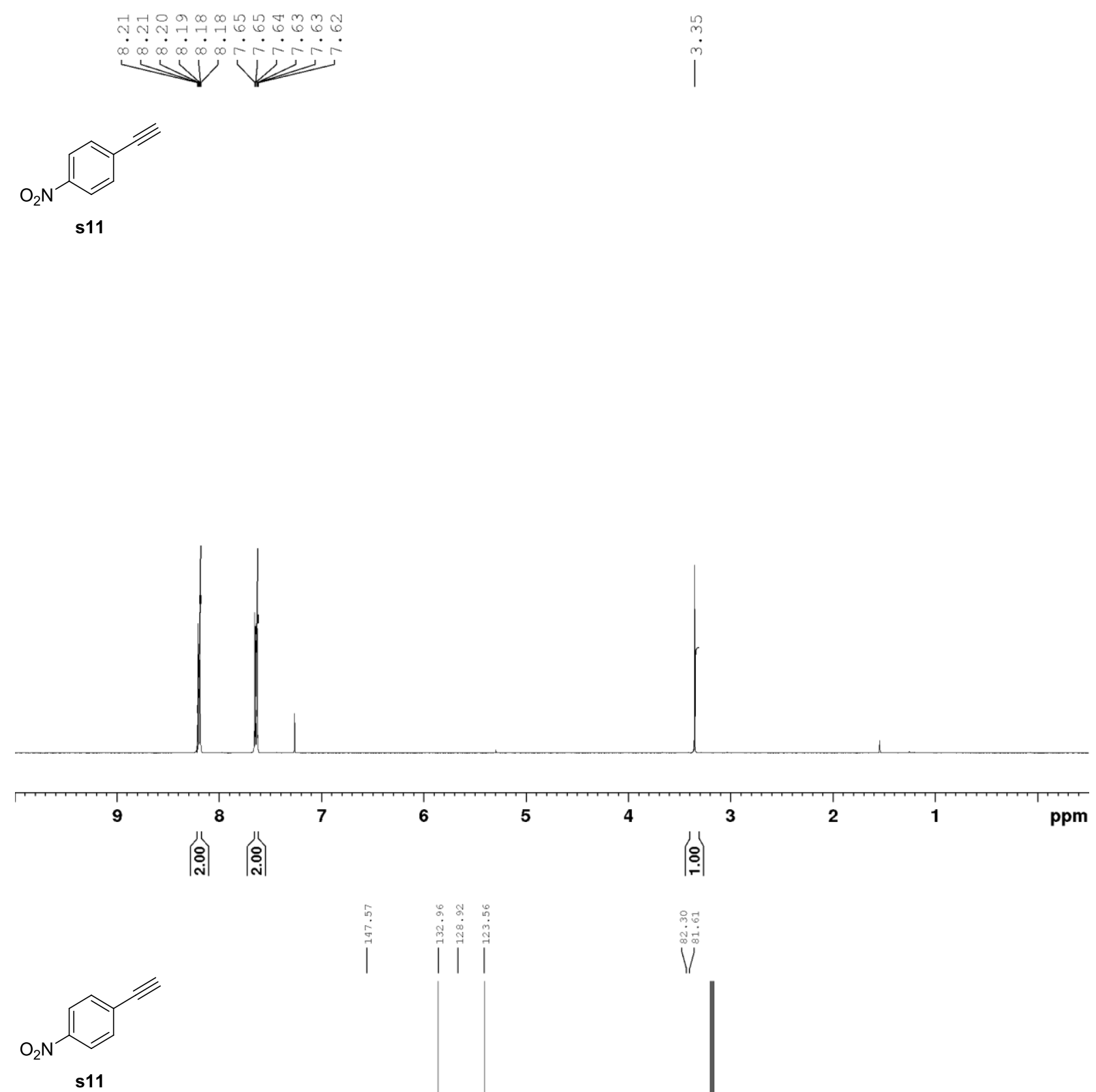

Vं

$\begin{array}{lllllllllllllllllllllll}210 & 200 & 190 & 180 & 170 & 160 & 150 & 140 & 130 & 120 & 110 & 100 & 90 & 80 & 70 & 60 & 50 & 40 & 30 & 20 & \mathrm{ppm}\end{array}$ 


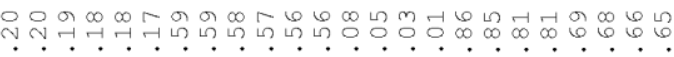

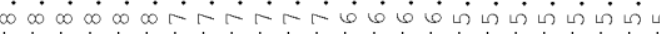

$+$

s12

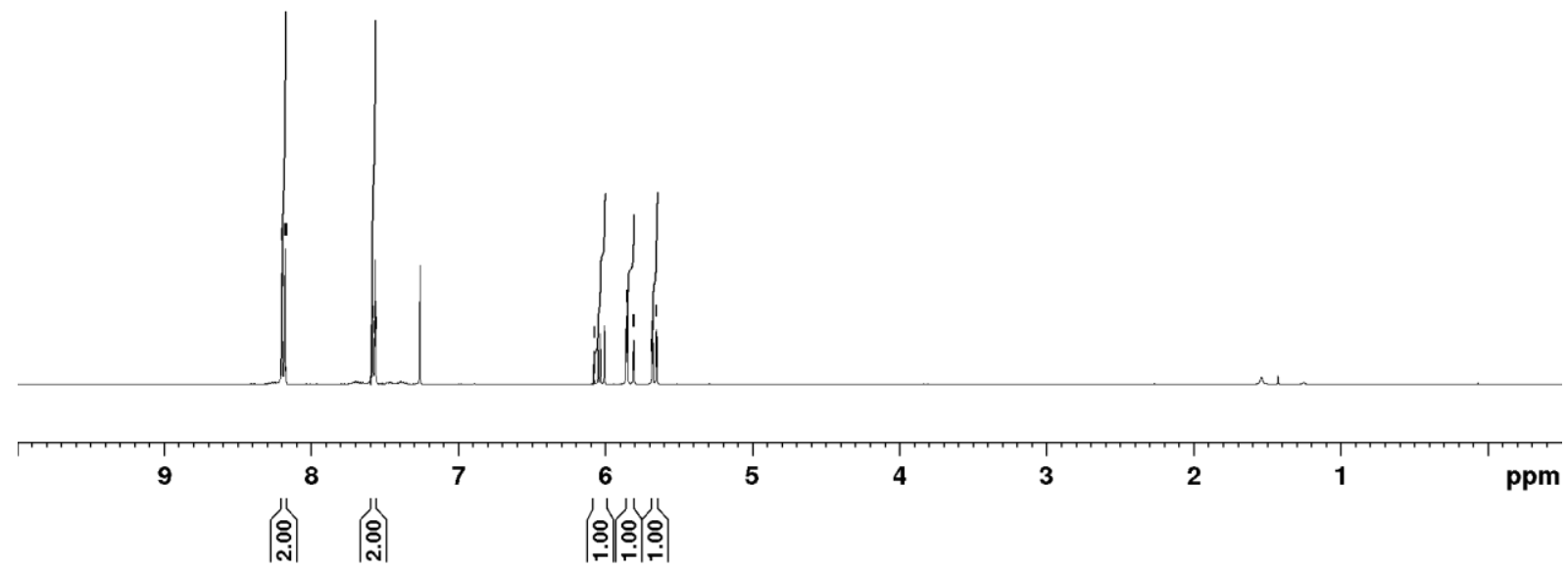

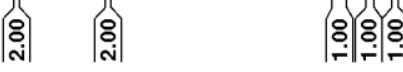

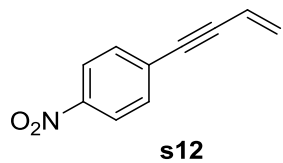

$\mid$
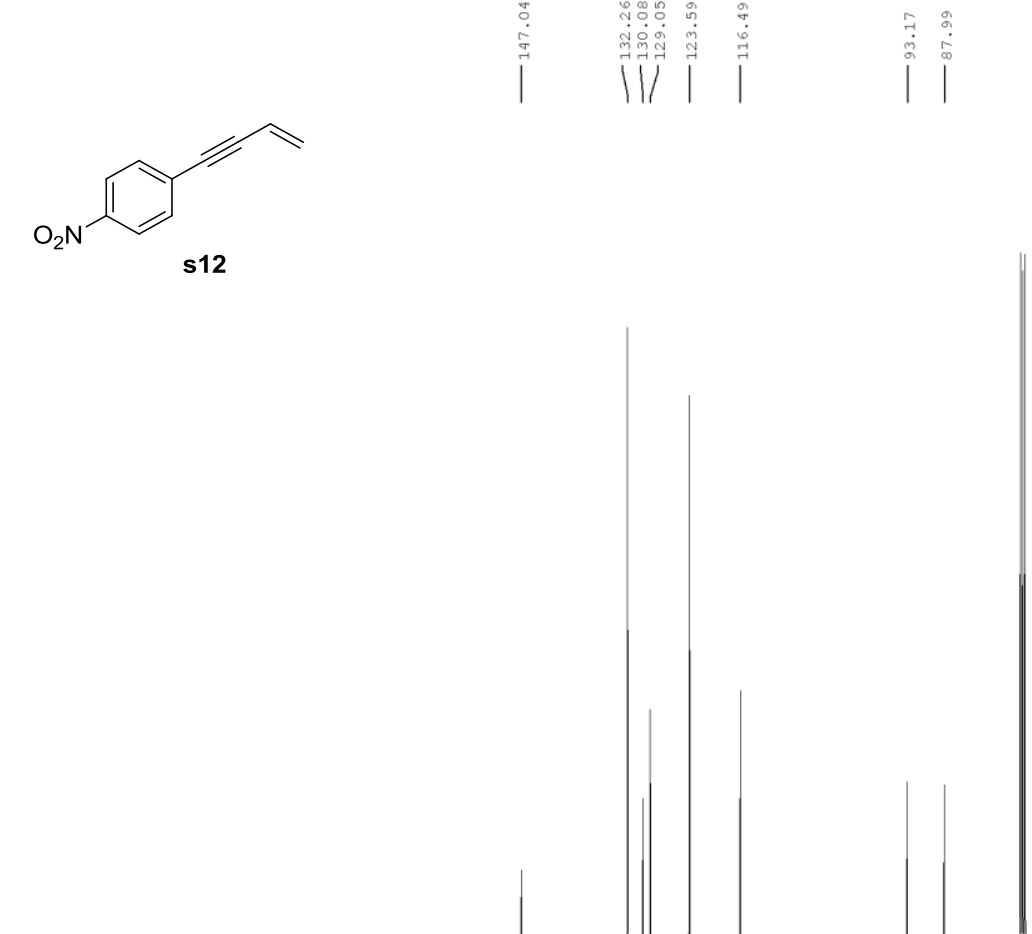

$\begin{array}{llllllllllllllllllllll}210 & 200 & 190 & 180 & 170 & 160 & 150 & 140 & 130 & 120 & 110 & 100 & 90 & 80 & 70 & 60 & 50 & 40 & 30 & 20 & \text { ppm }\end{array}$ 

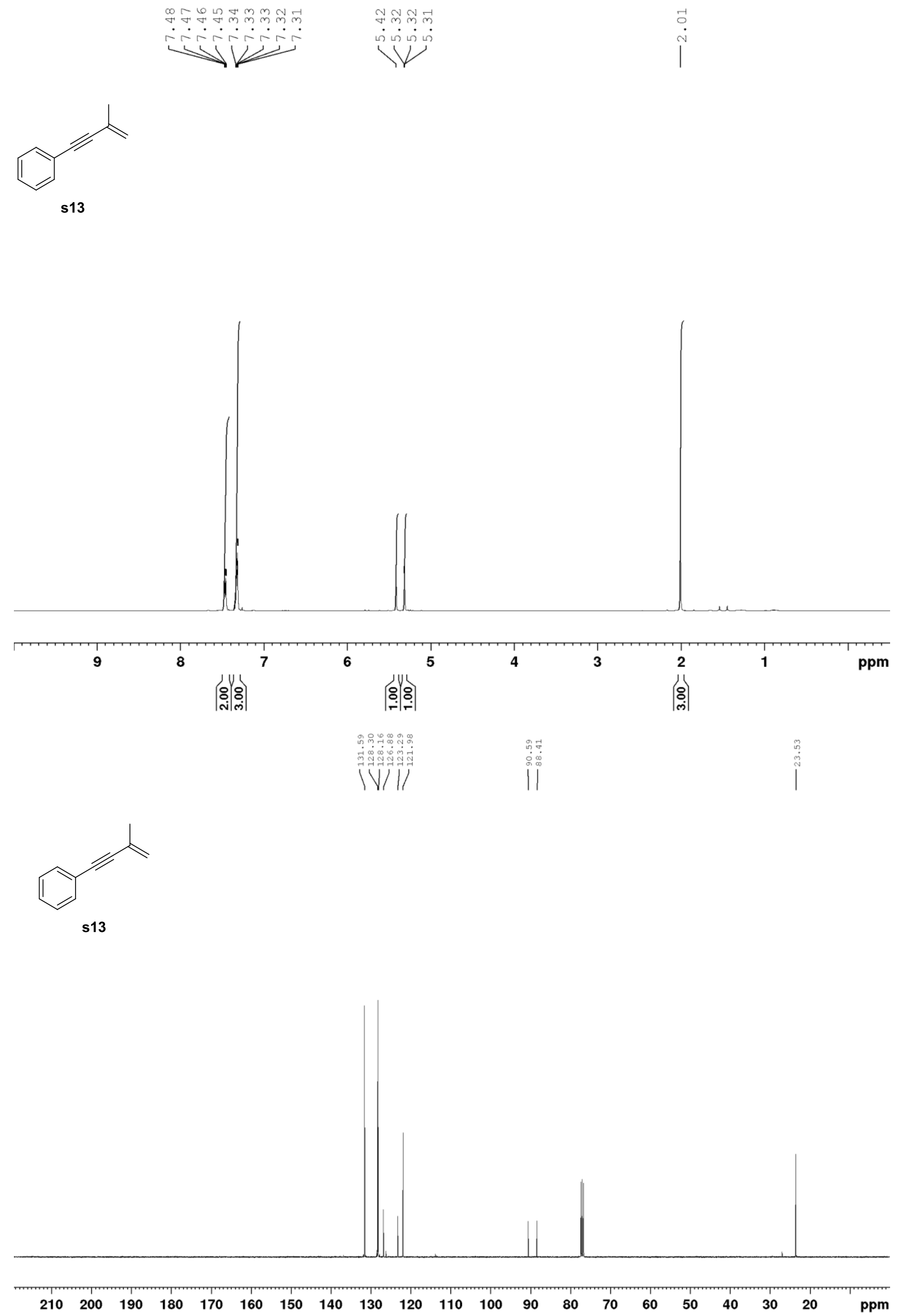


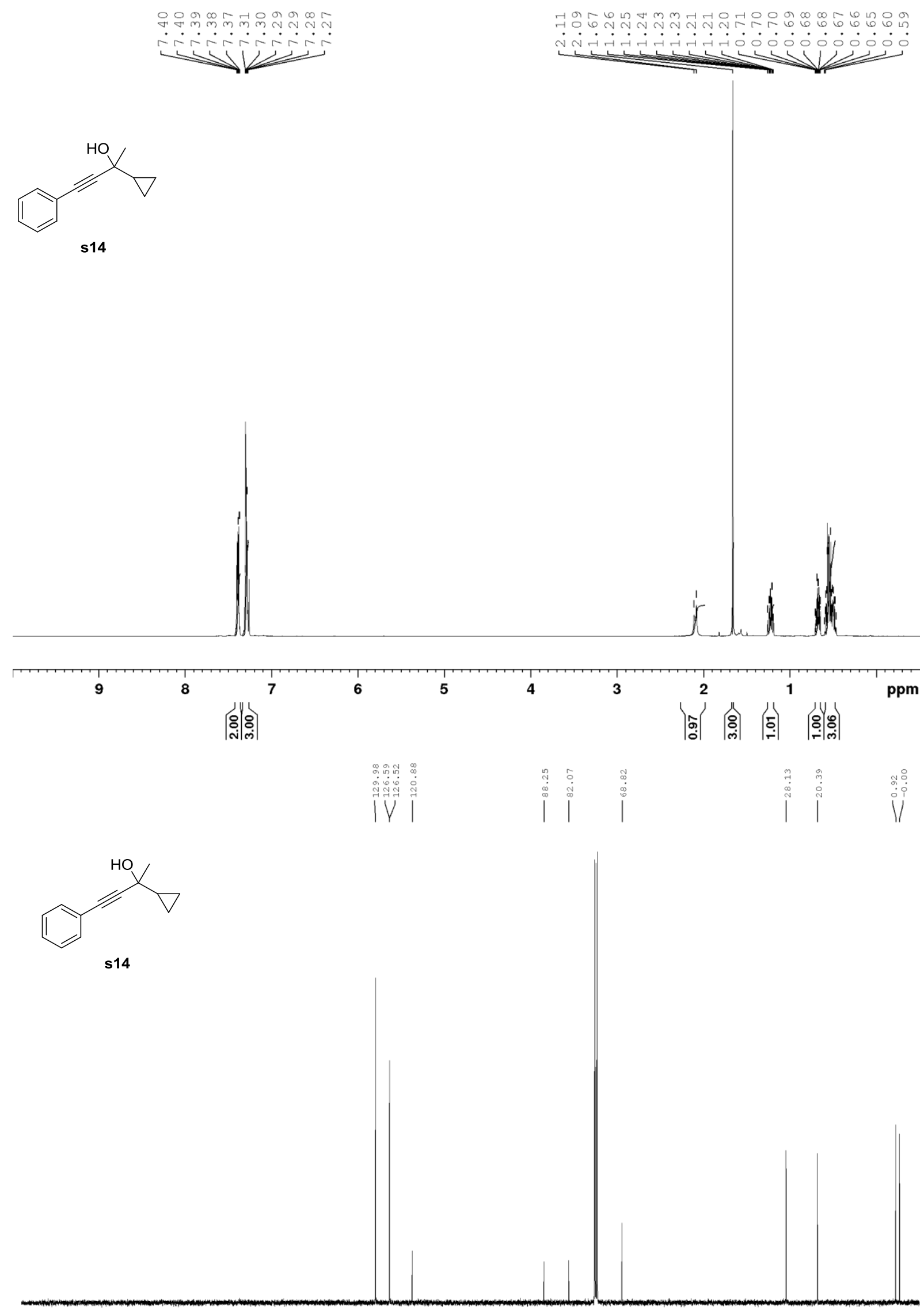

$\begin{array}{llllllllllllllllllllll}210 & 200 & 190 & 180 & 170 & 160 & 150 & 140 & 130 & 120 & 110 & 100 & 90 & 80 & 70 & 60 & 50 & 40 & 30 & 20 & 10 & \mathrm{ppm}\end{array}$ 


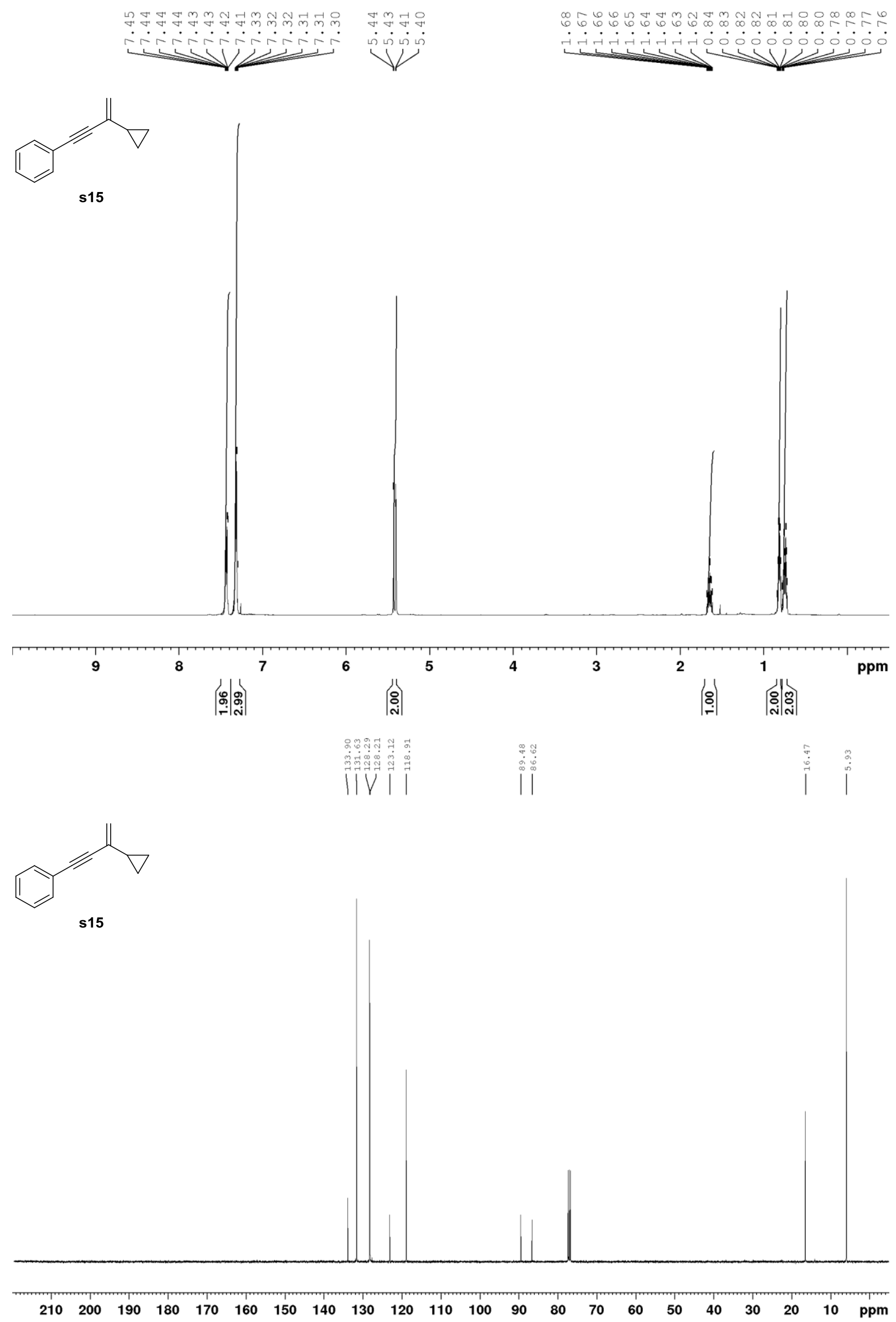



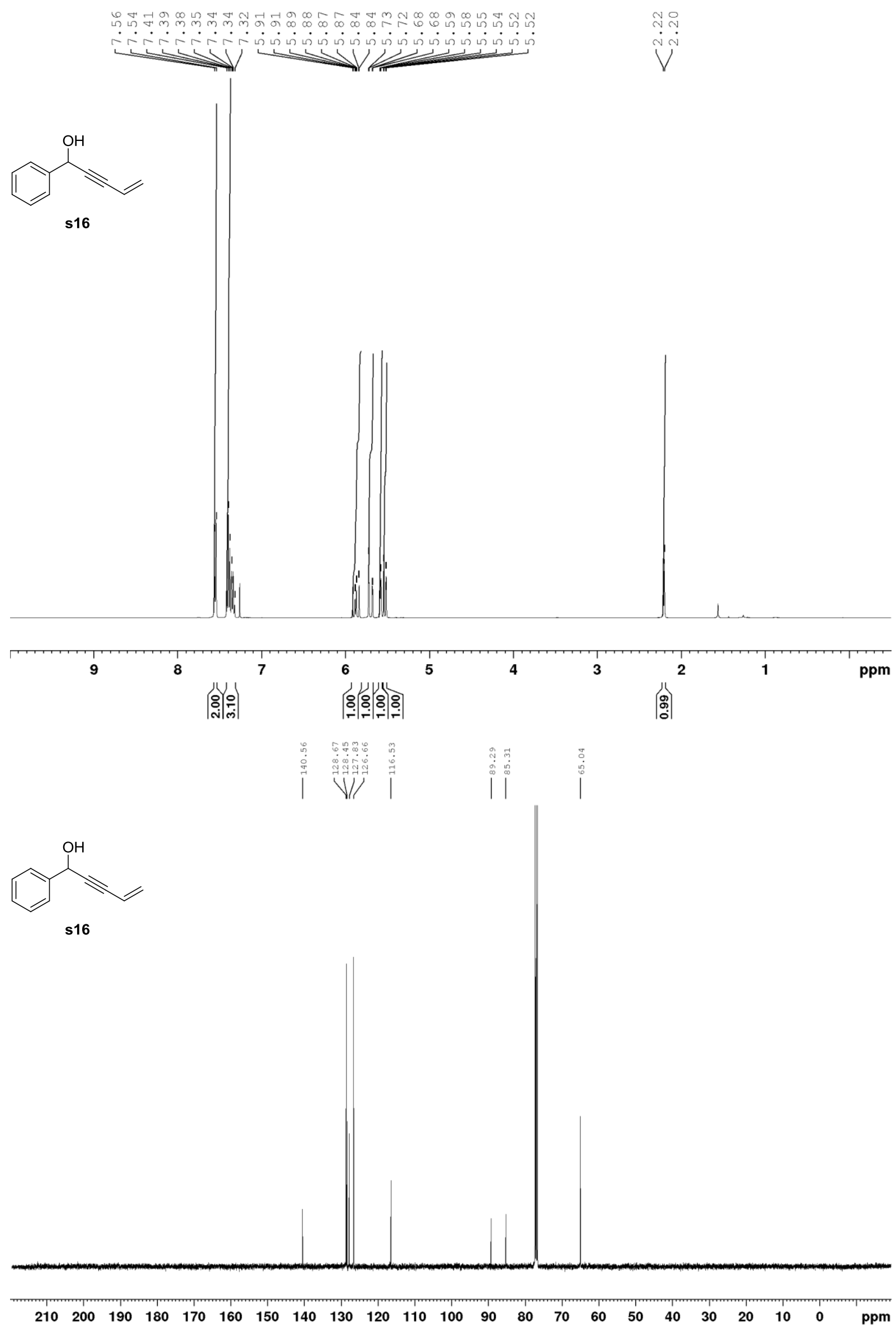

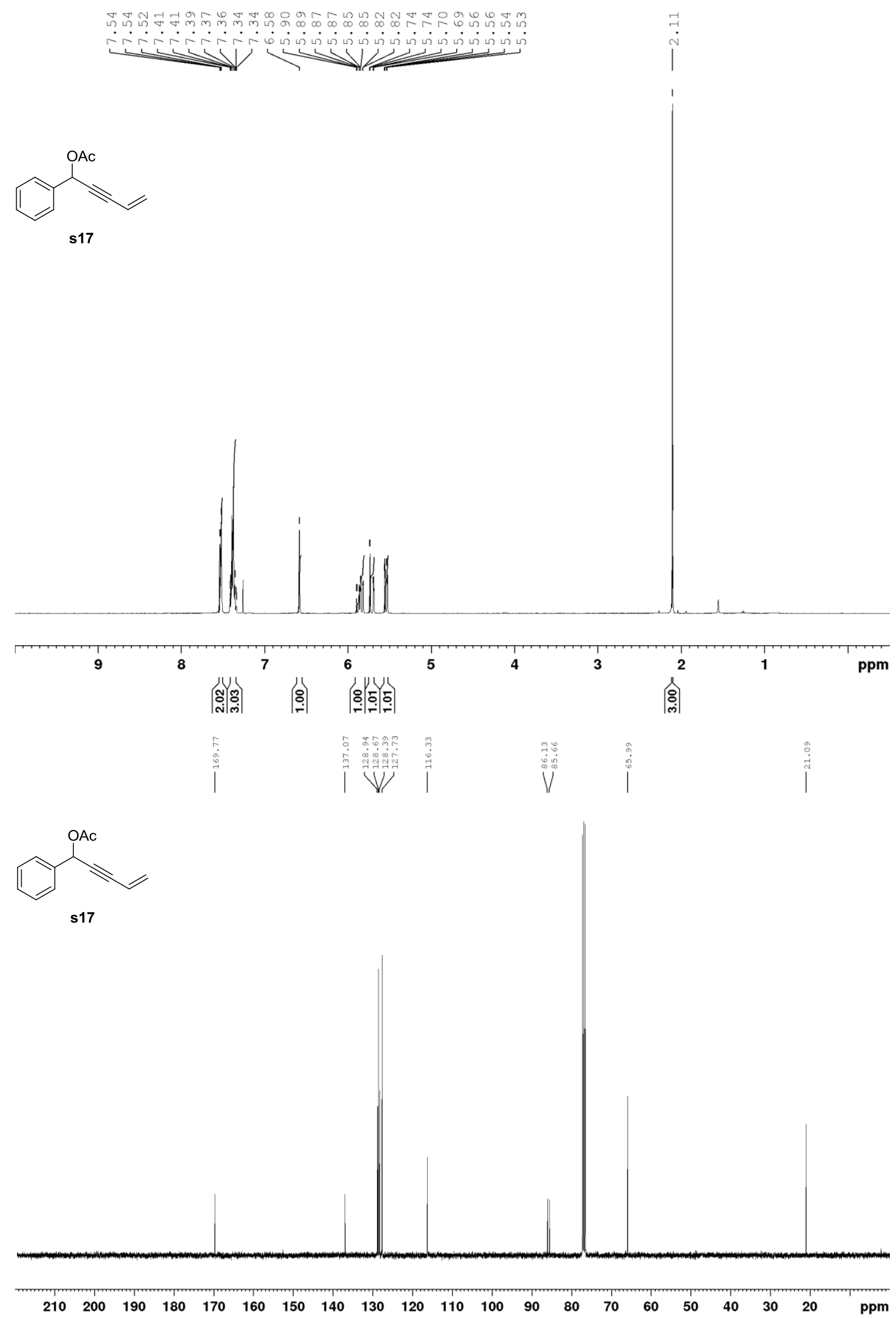


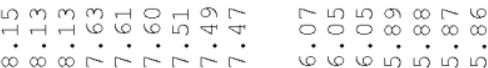

Yon vifir

$\underbrace{O}_{s 18}$

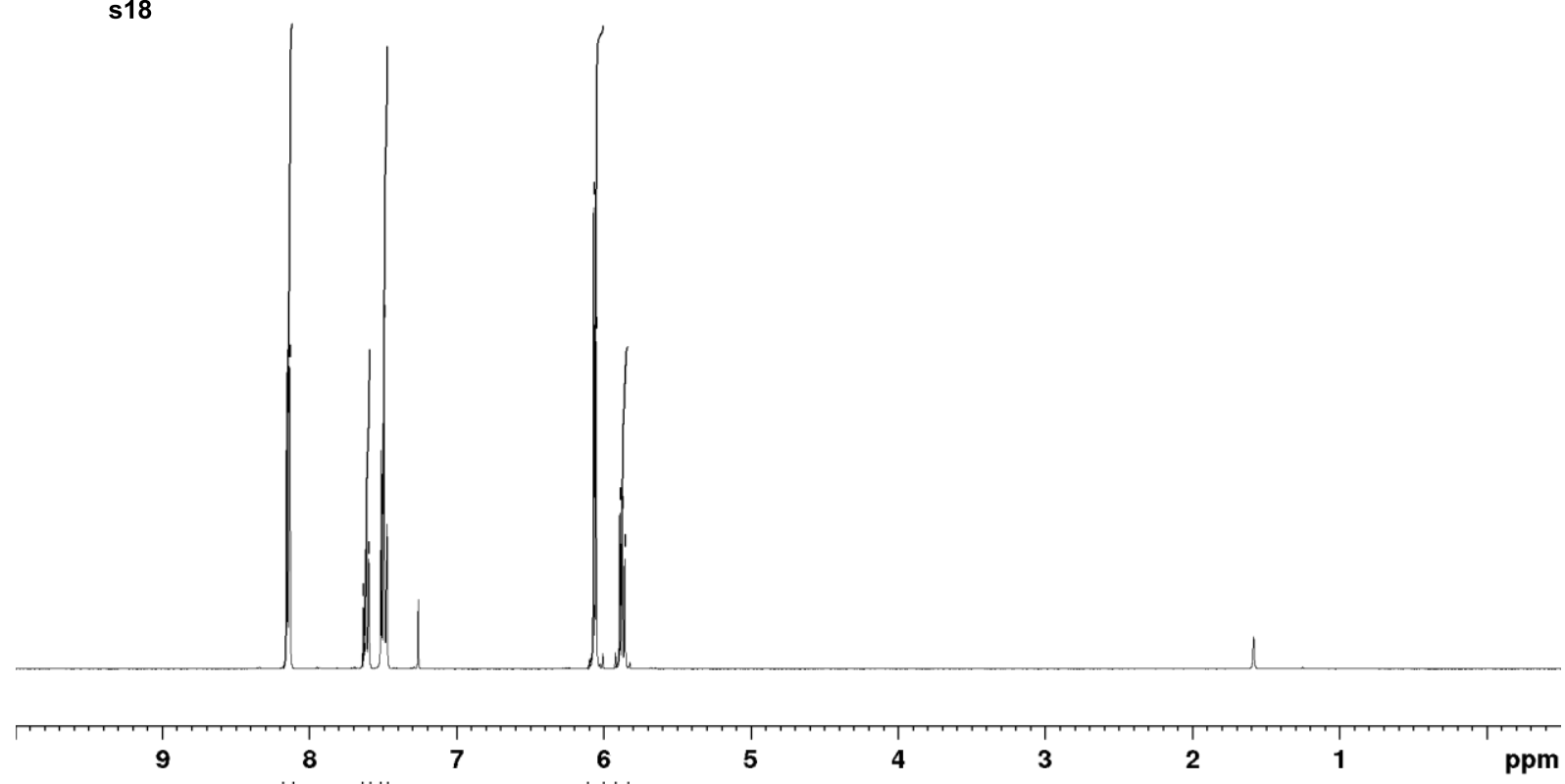

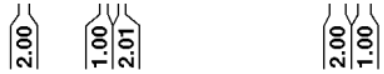
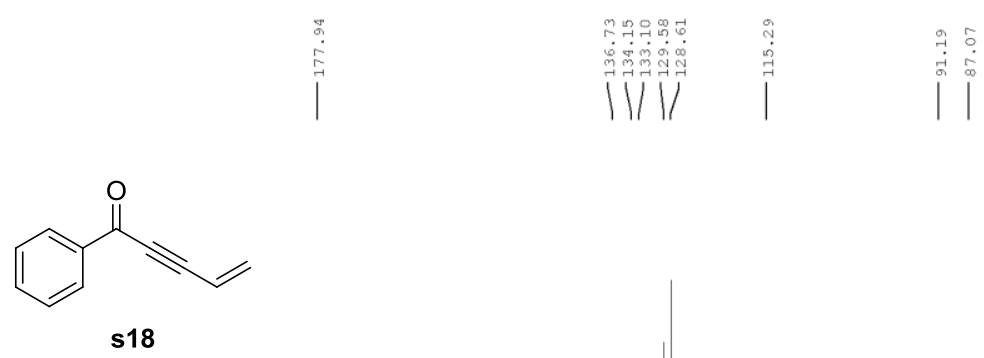

$|1|$

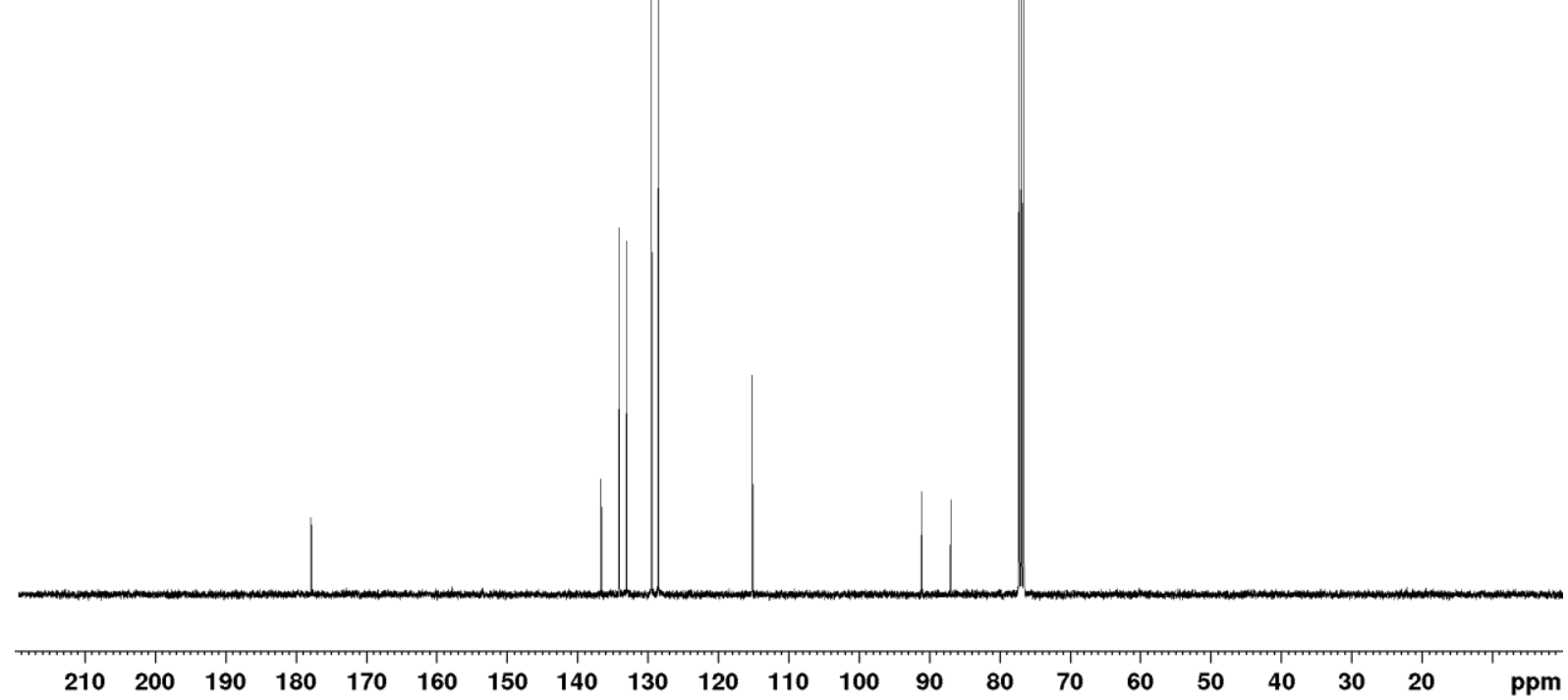



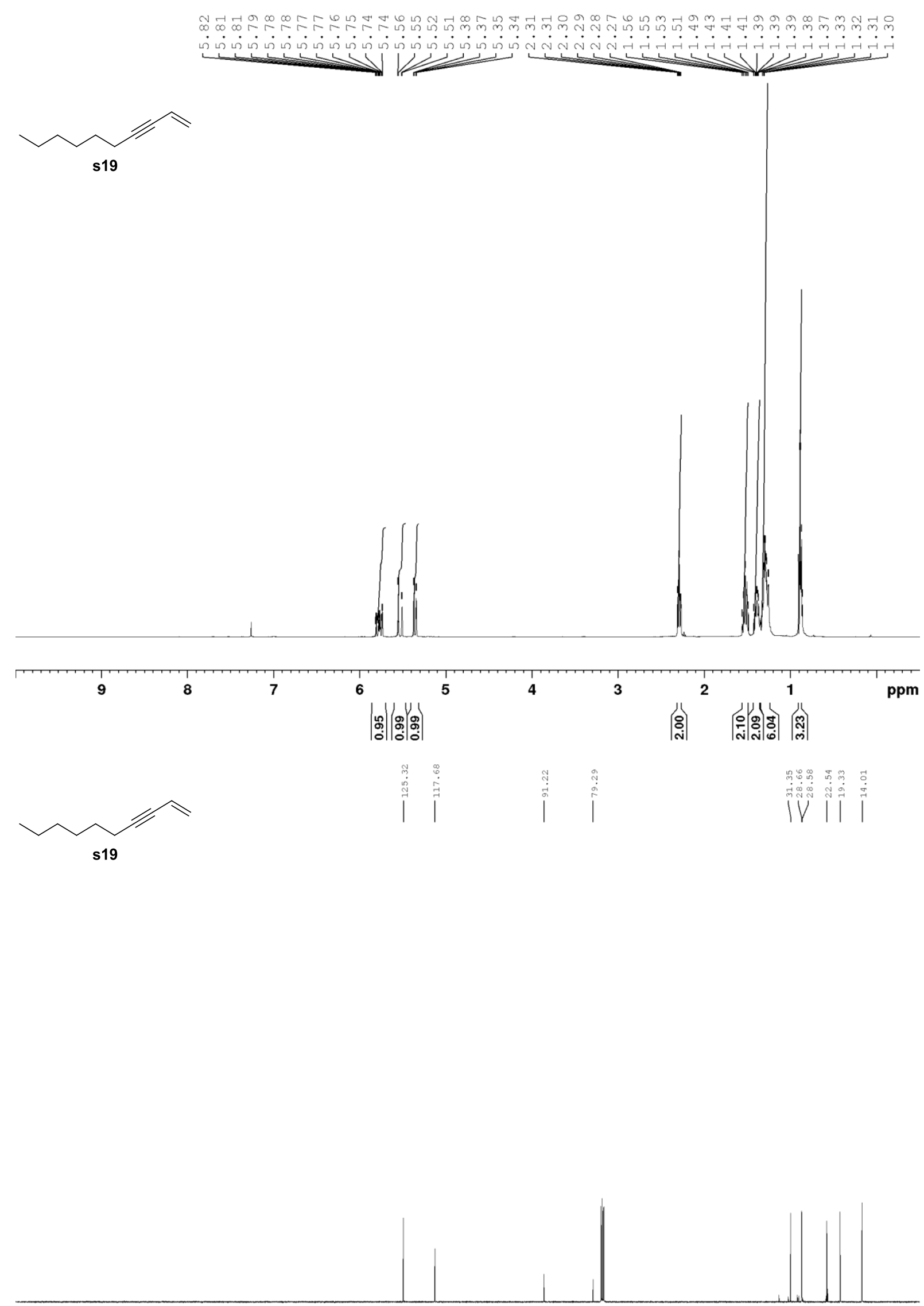

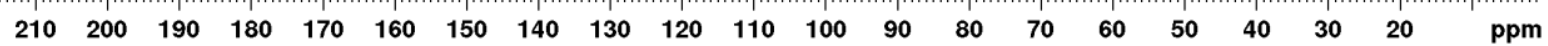




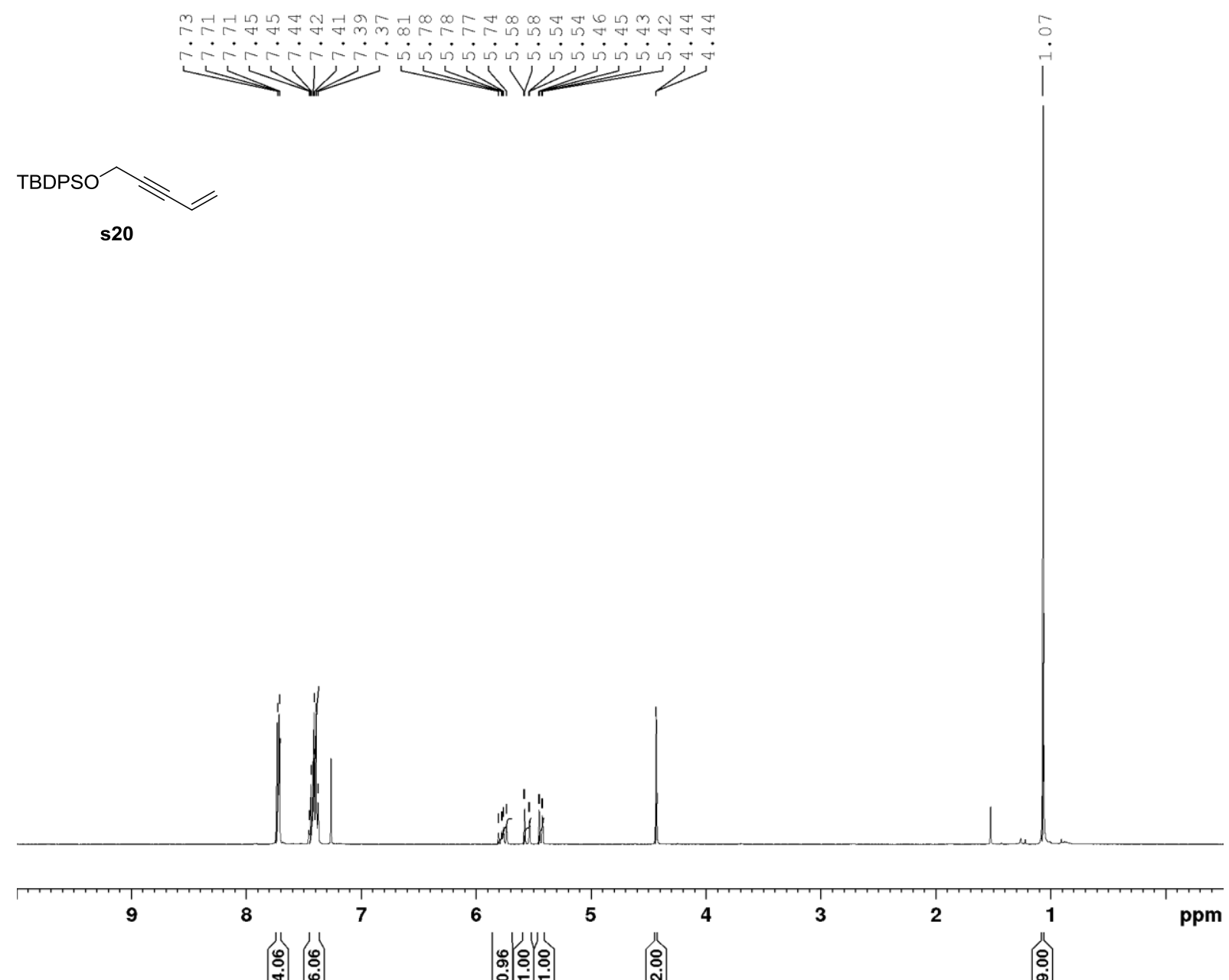

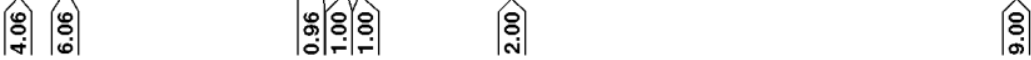
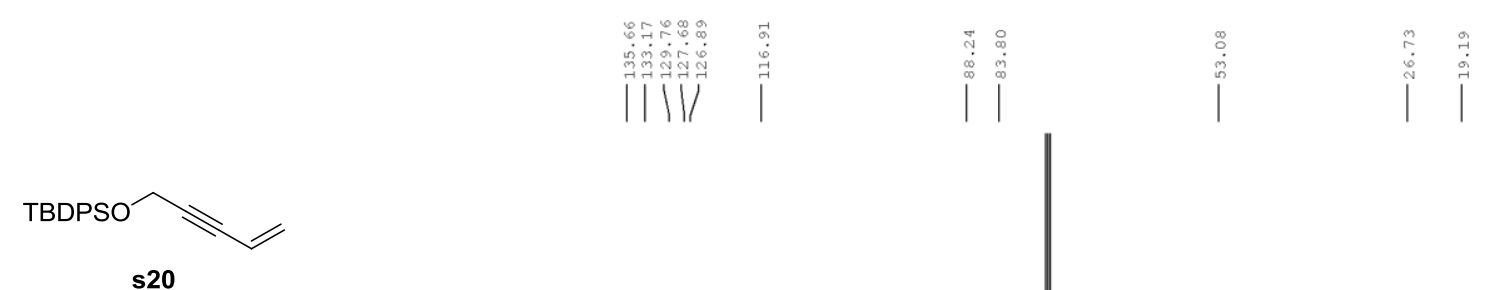

s20

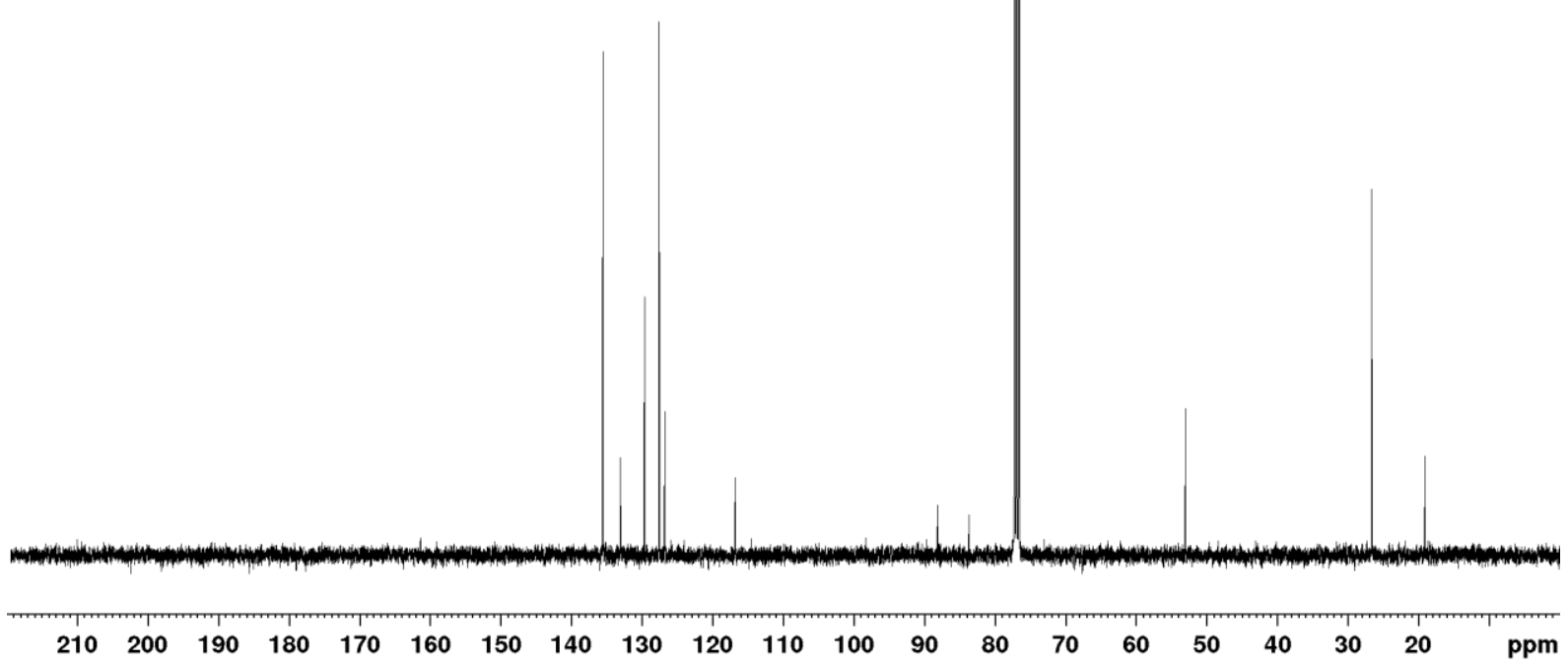




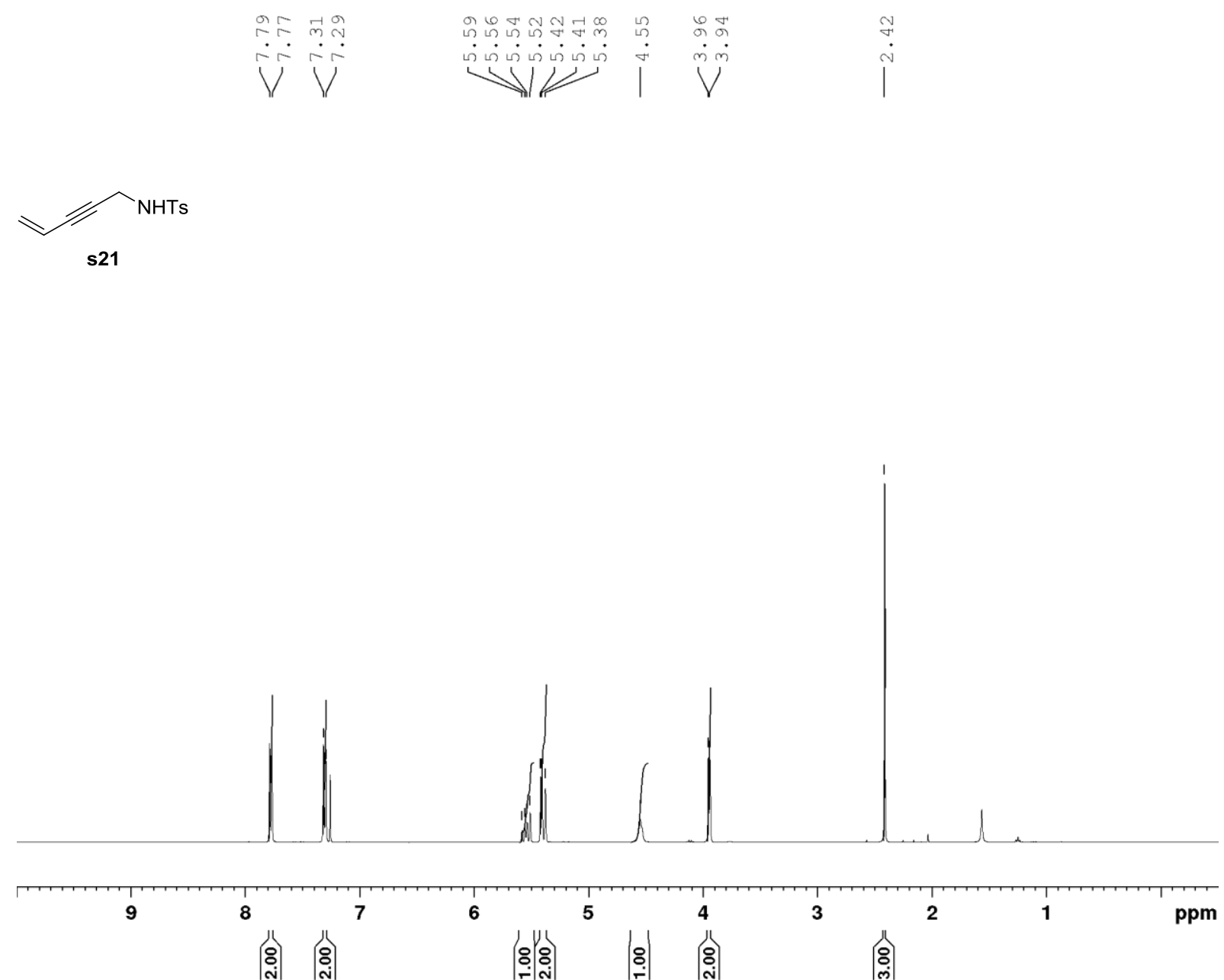

|
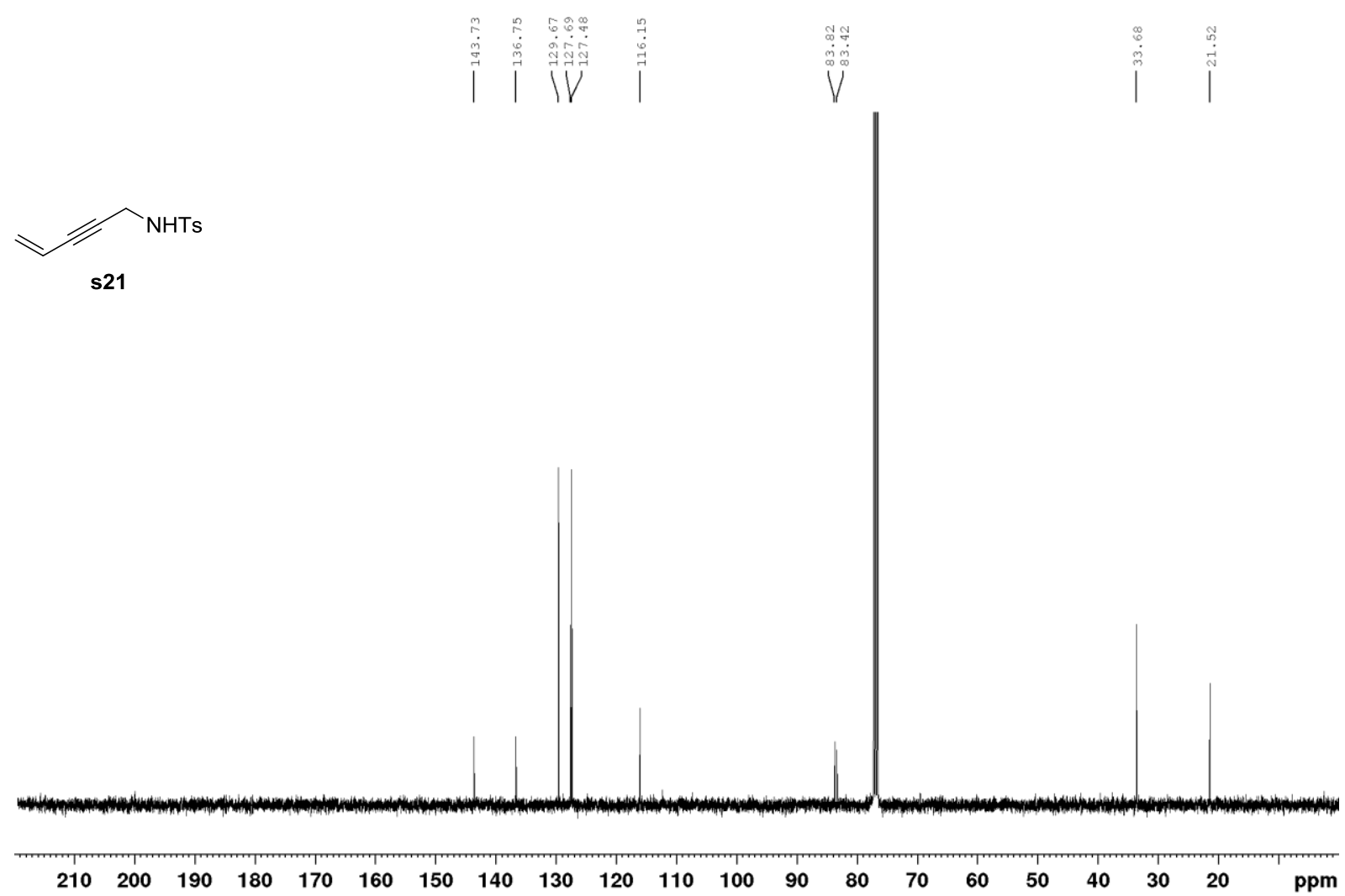

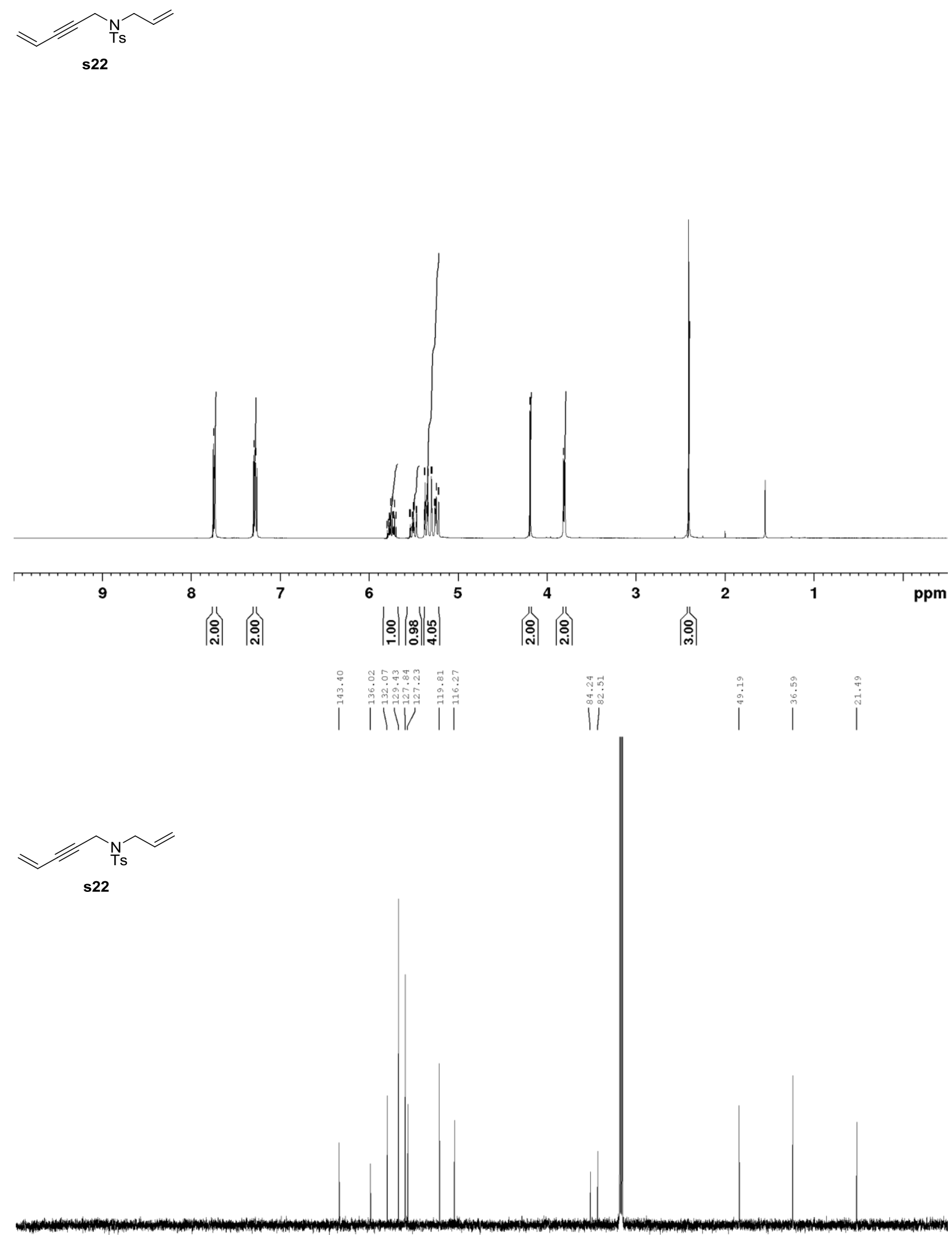

$\begin{array}{lllllllllllllllllllll}210 & 200 & 190 & 180 & 170 & 160 & 150 & 140 & 130 & 120 & 110 & 100 & 90 & 80 & 70 & 60 & 50 & 40 & 30 & 20 & \mathrm{ppm}\end{array}$ 


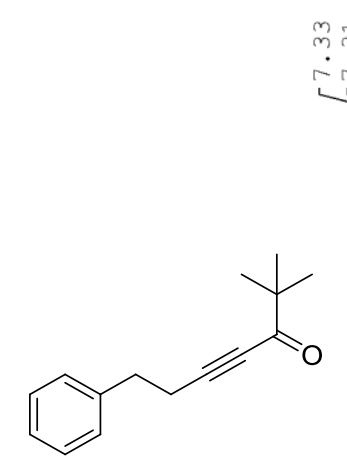

身

정하마뭉

บivivi

s23
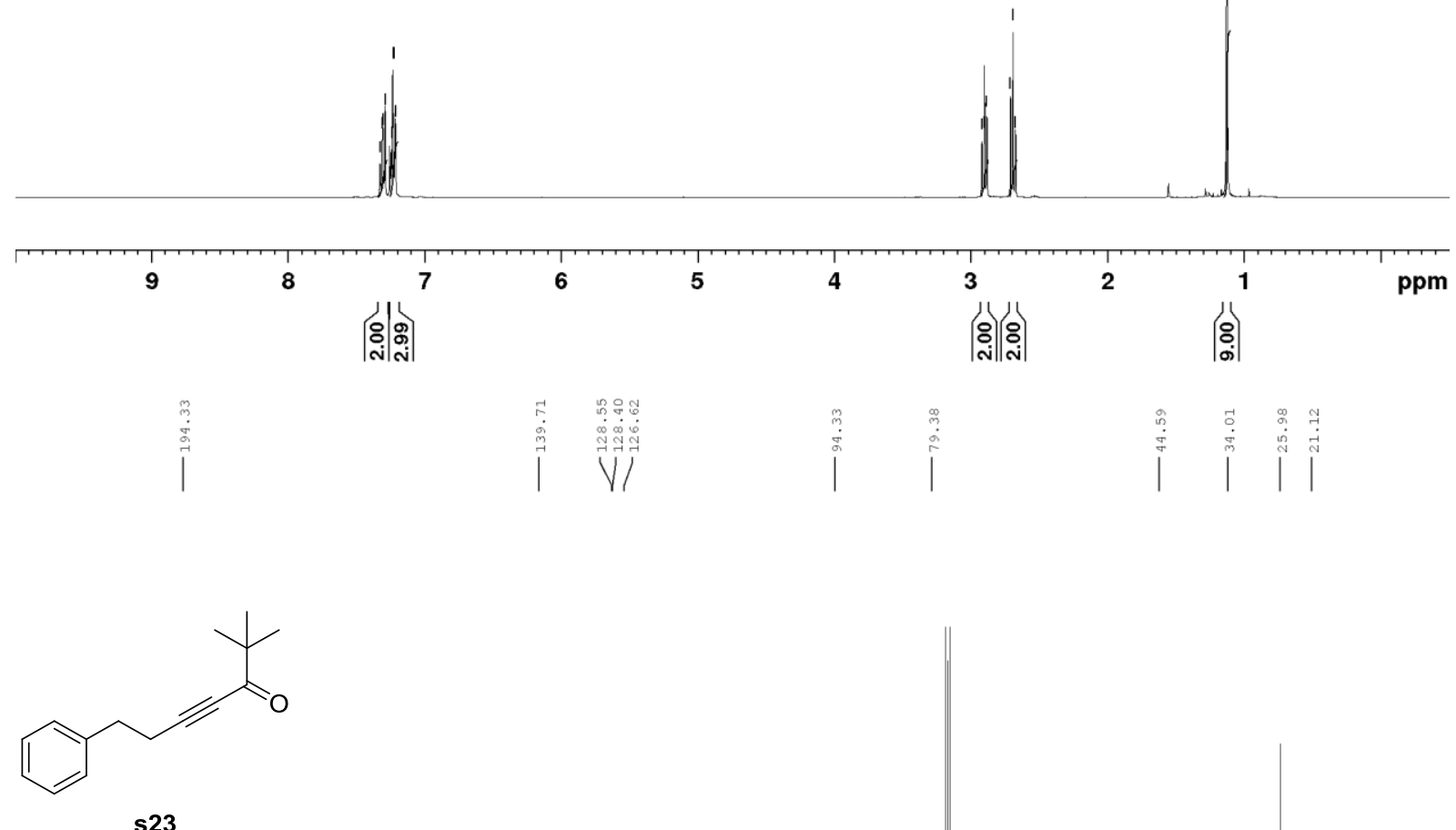

523

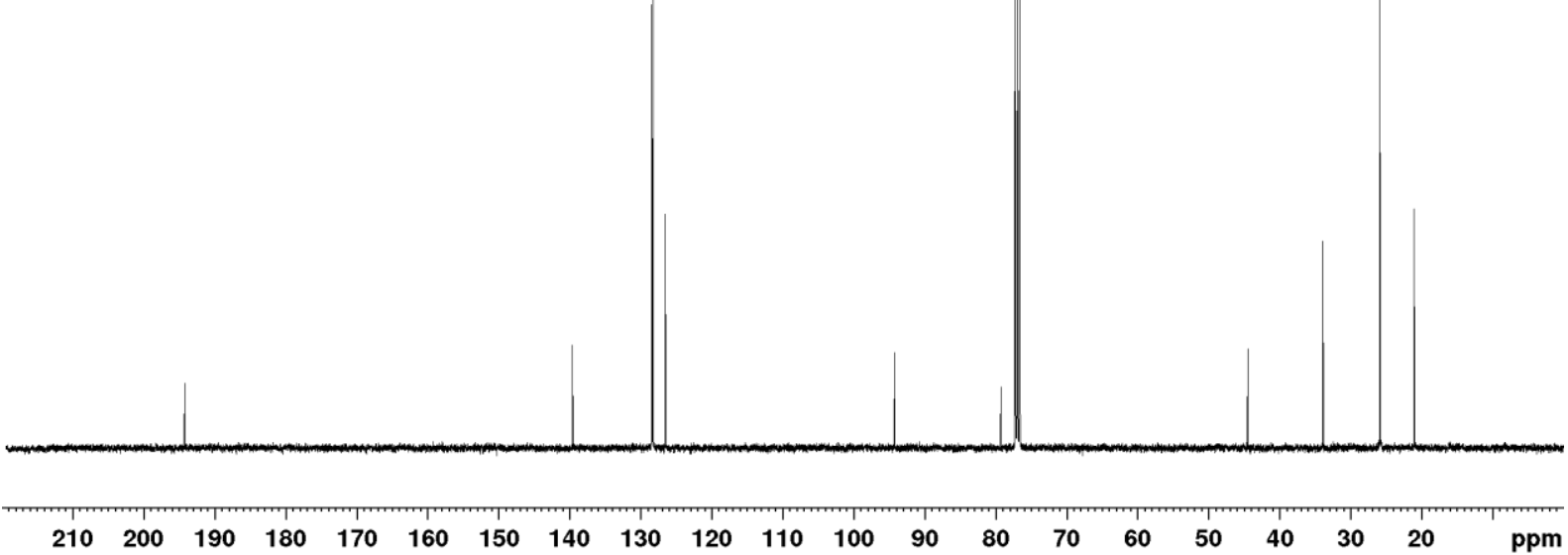



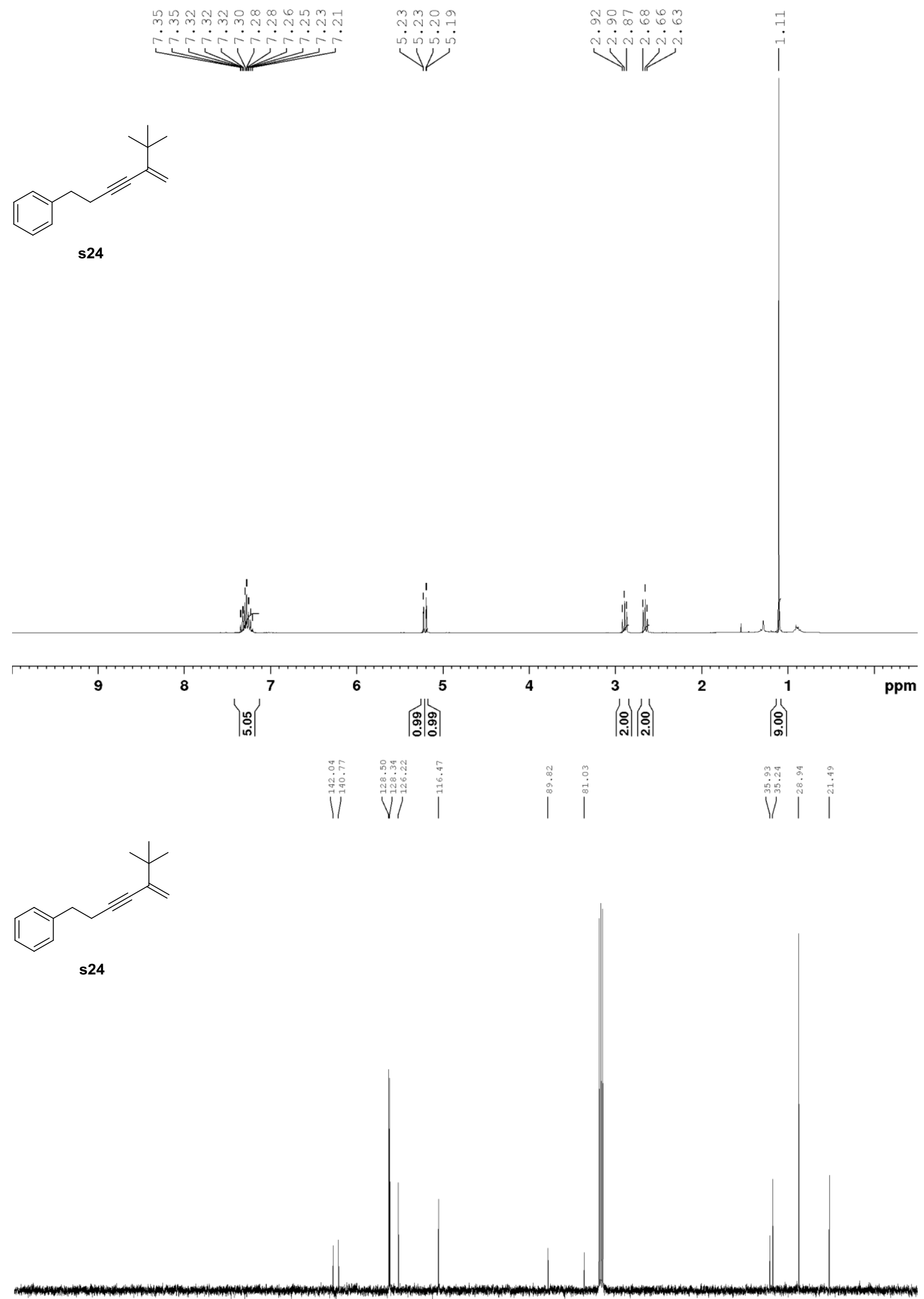

$\begin{array}{lllllllllllllllllllll}210 & 200 & 190 & 180 & 170 & 160 & 150 & 140 & 130 & 120 & 110 & 100 & 90 & 80 & 70 & 60 & 50 & 40 & 30 & 20 & \mathrm{ppm}\end{array}$ 

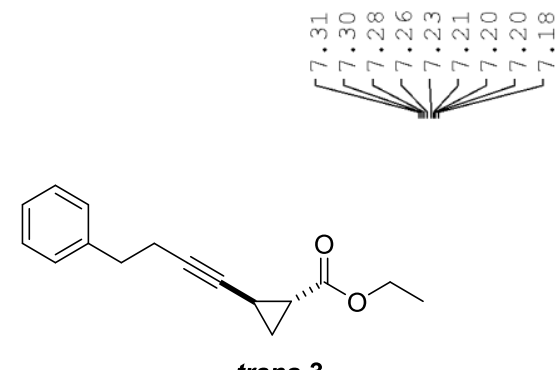

trans-3
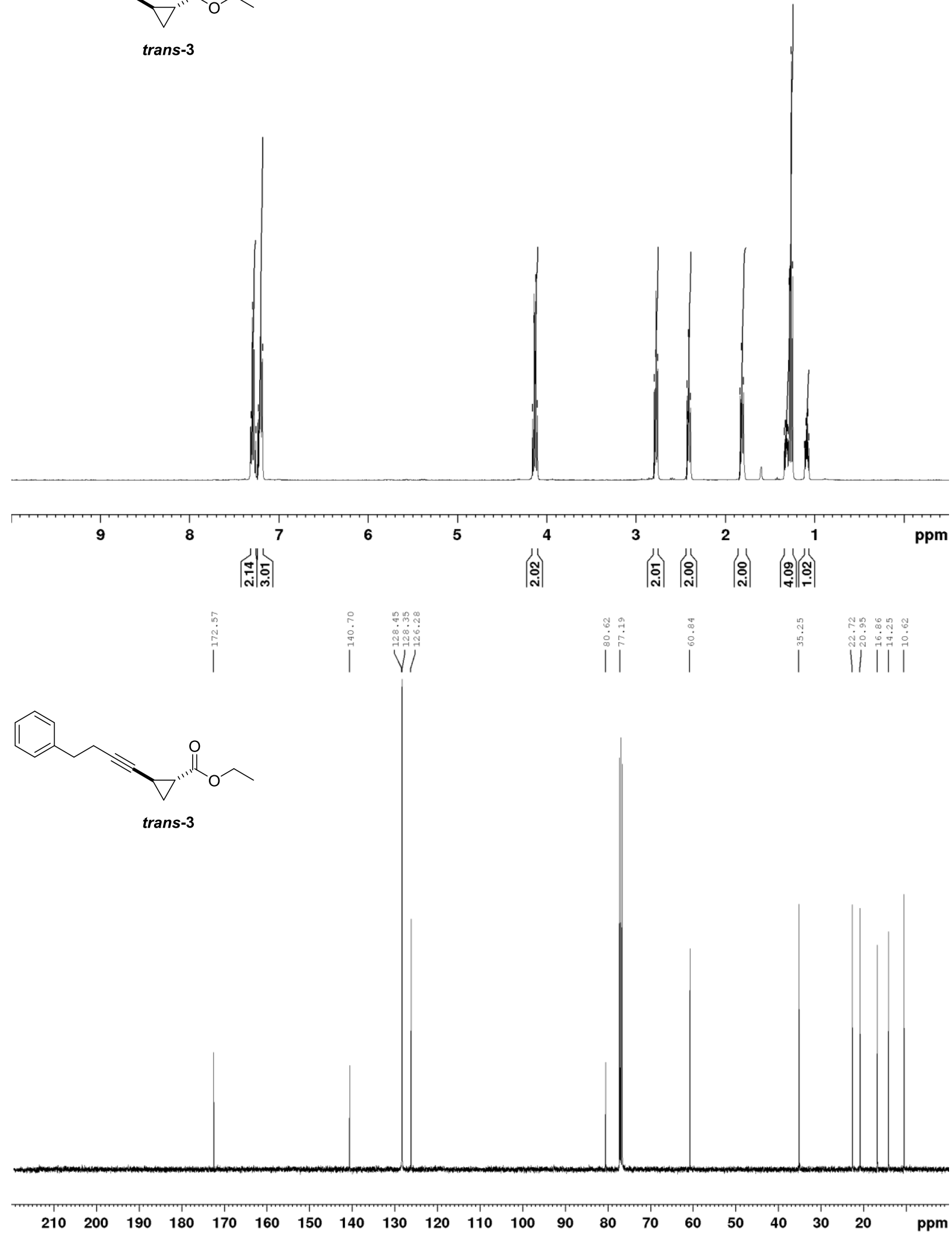
$\underbrace{-10}$
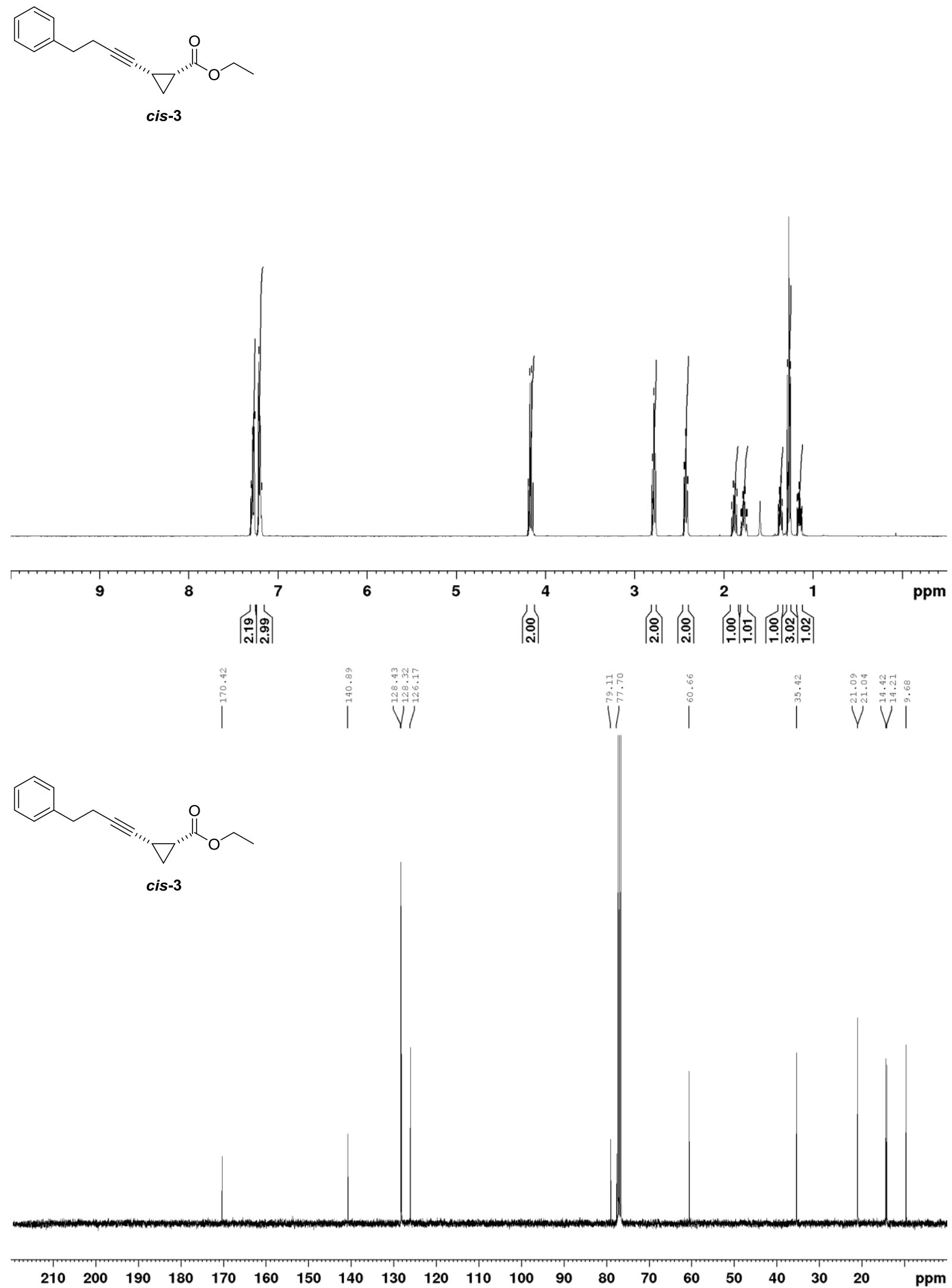

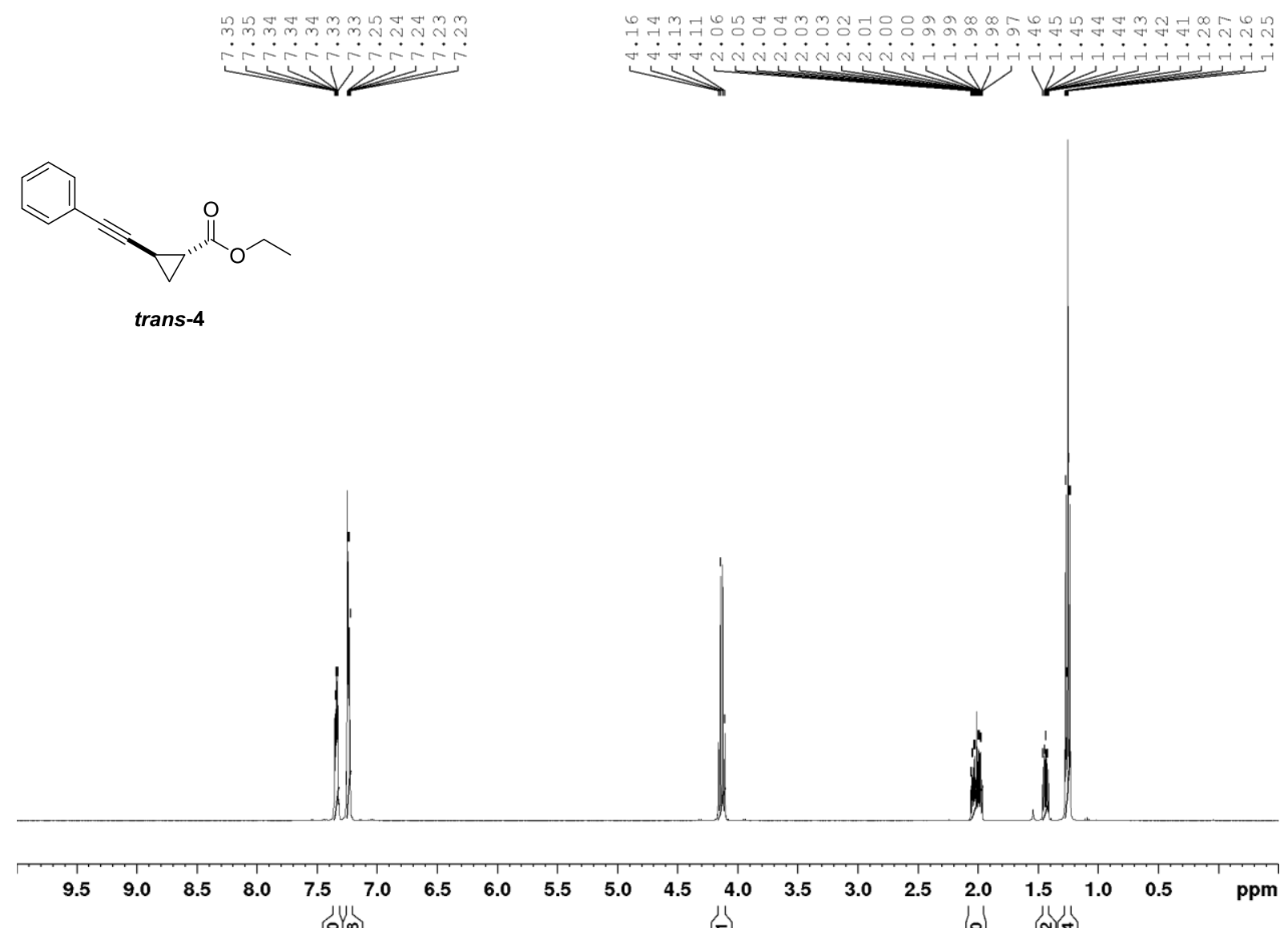

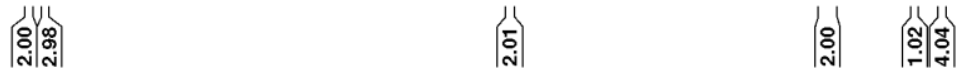
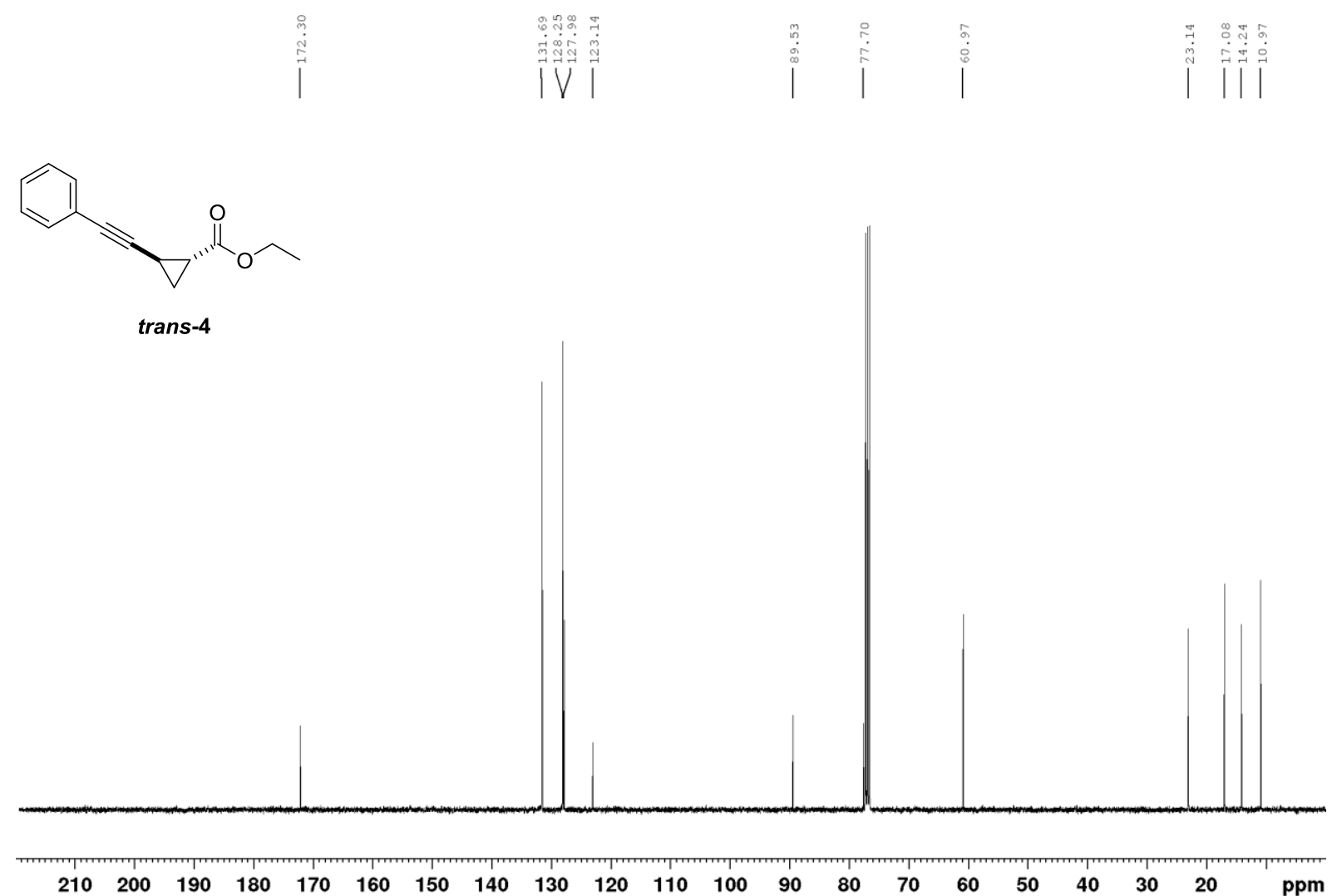

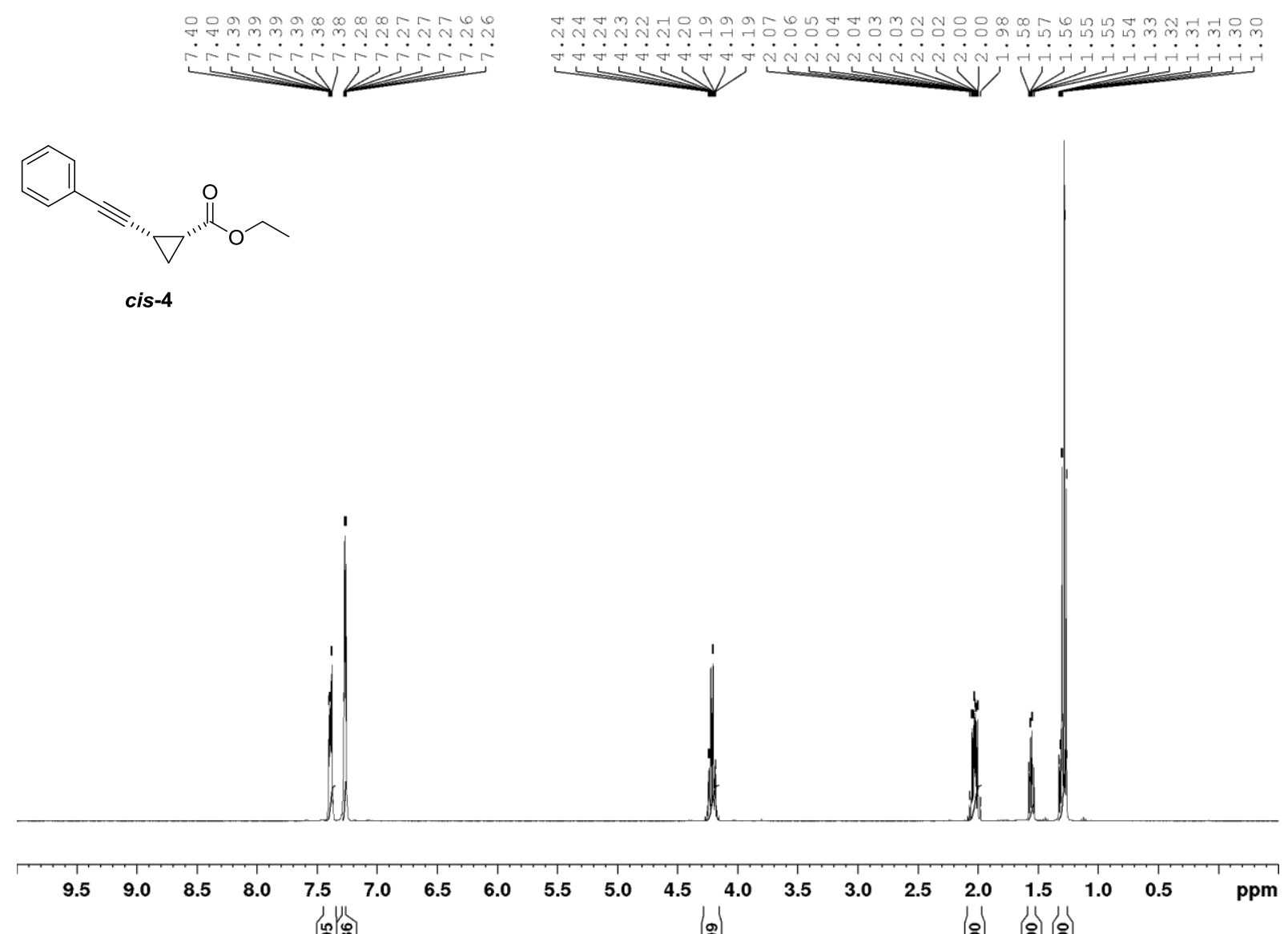

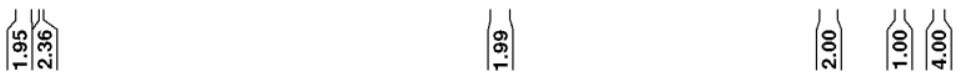

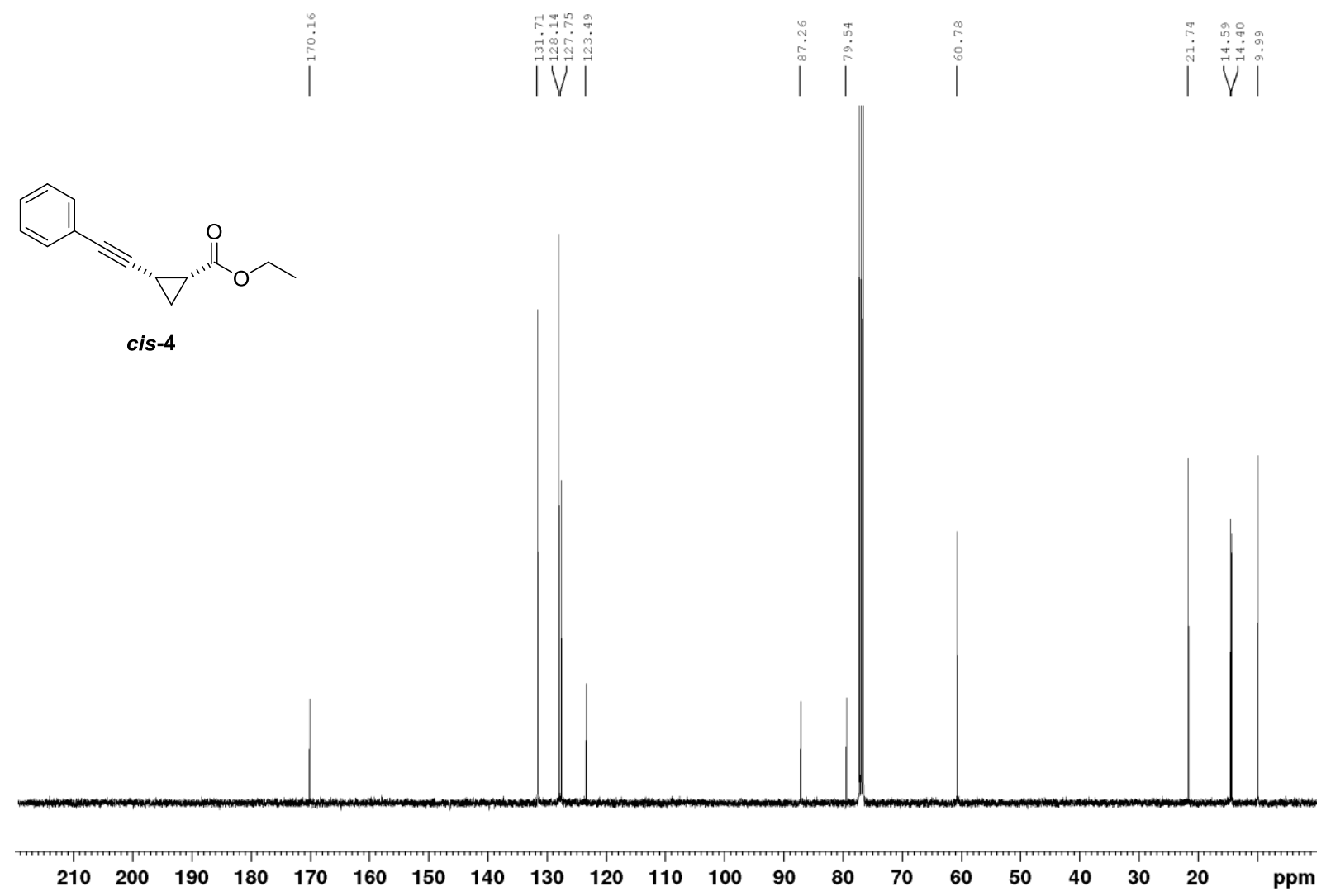




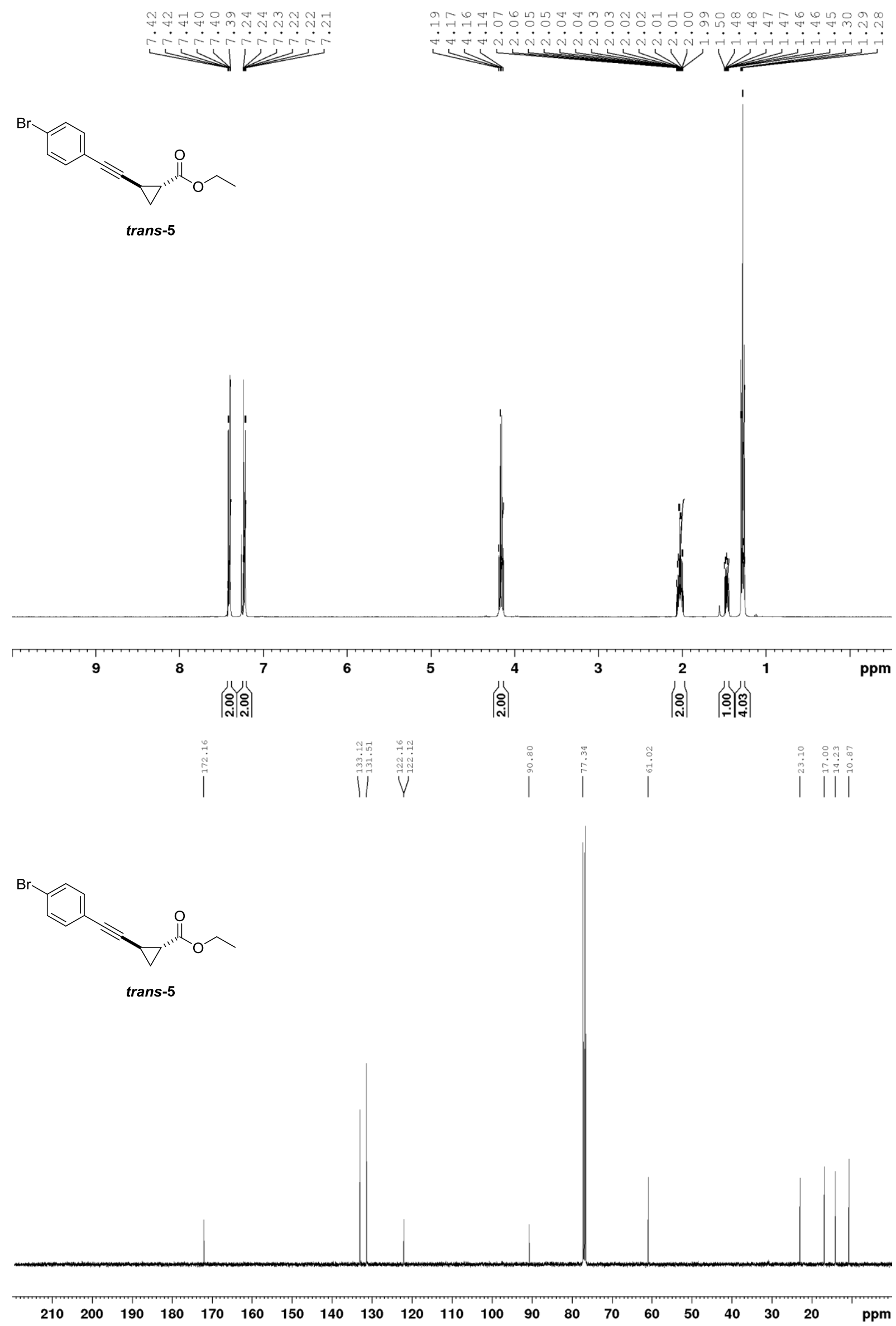




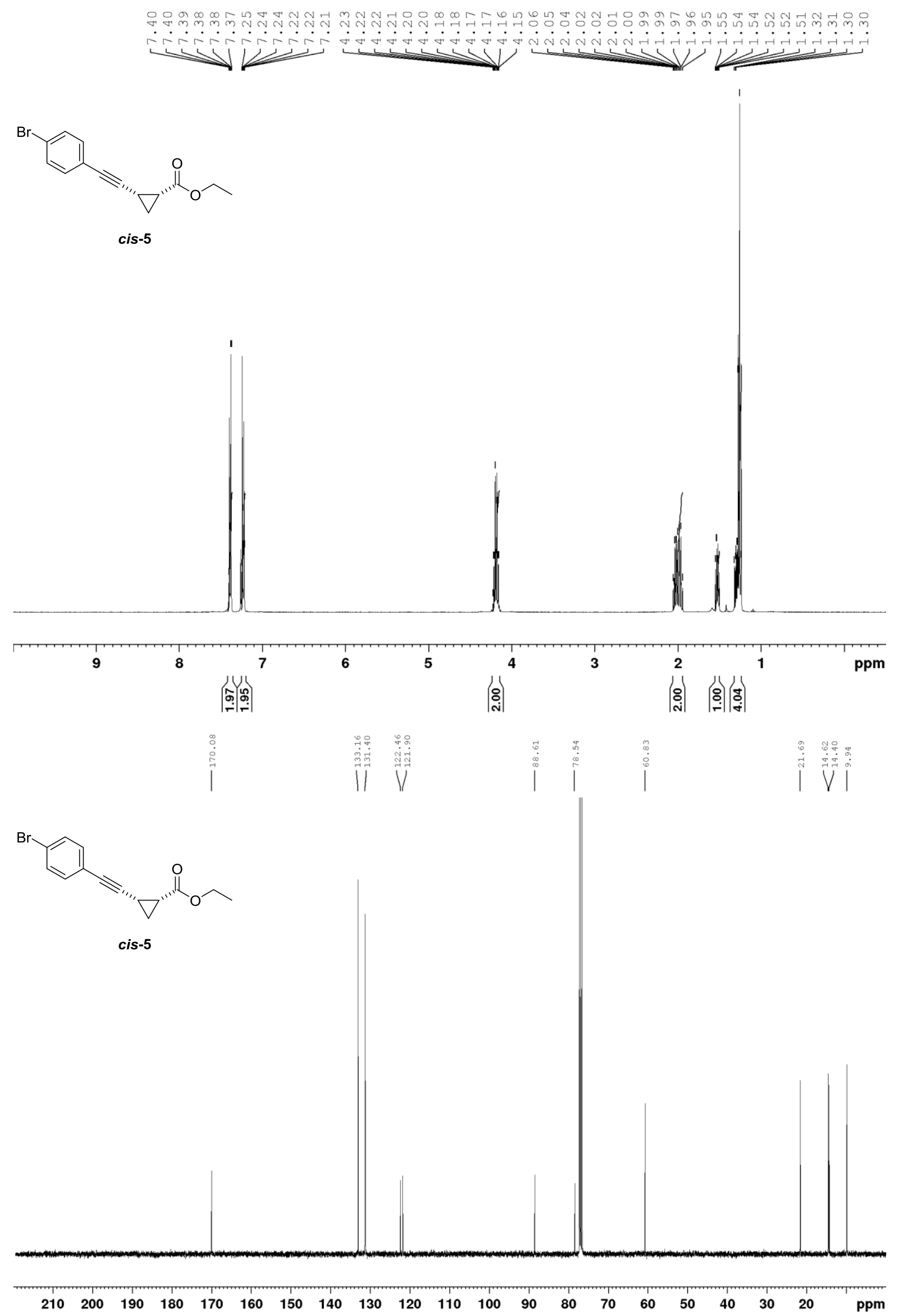




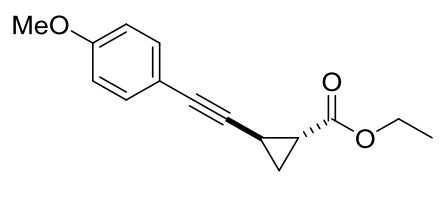

trans-6

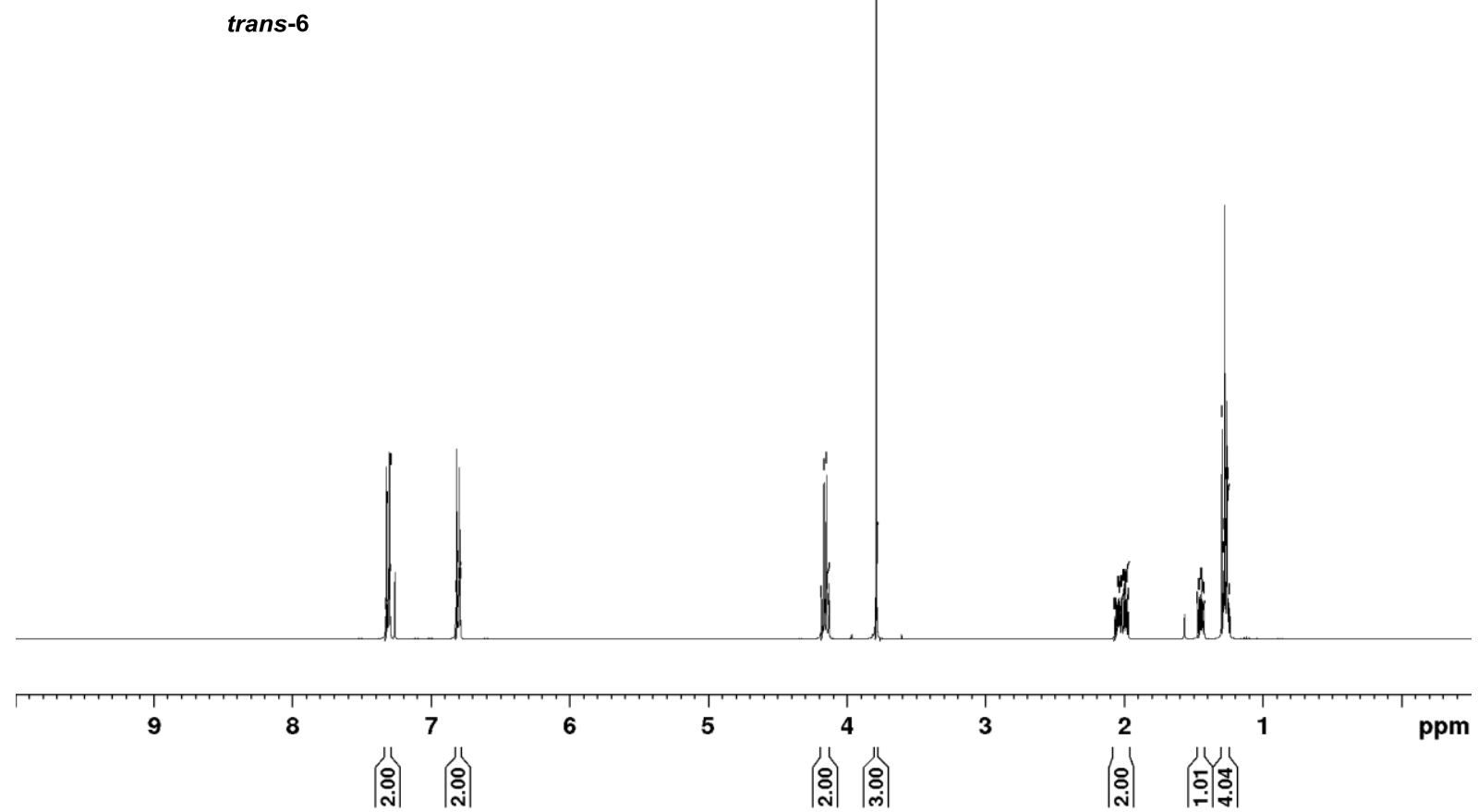

|
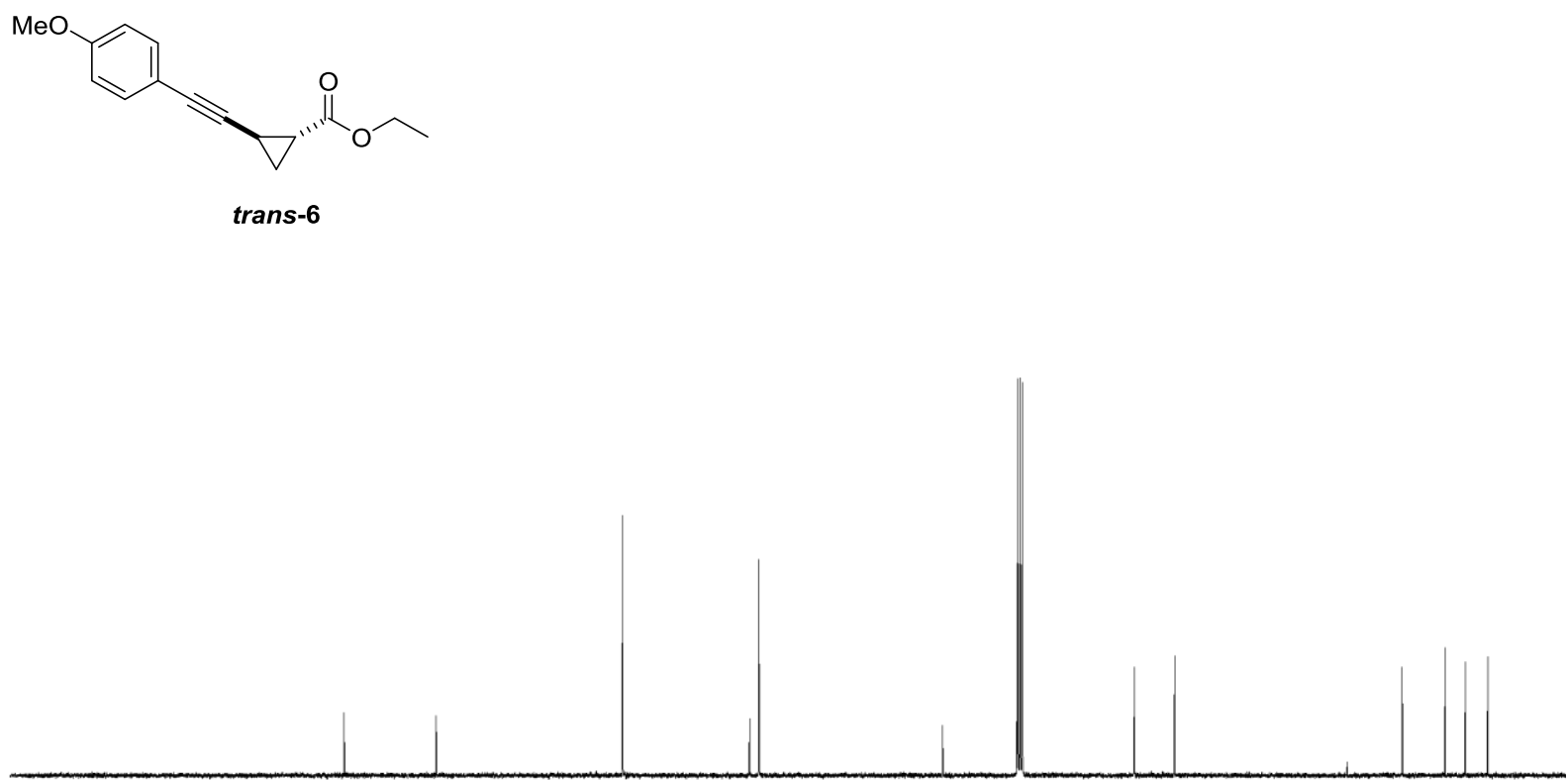

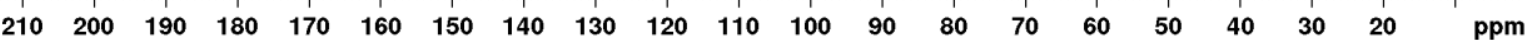




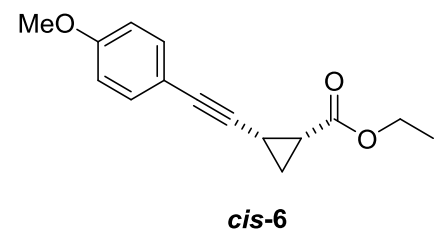

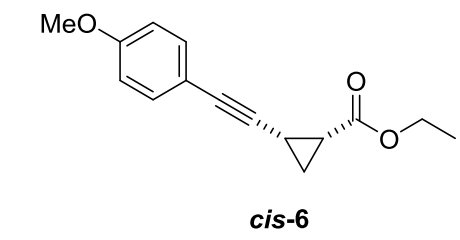

cis-6

6

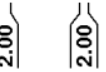

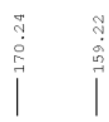

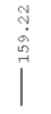

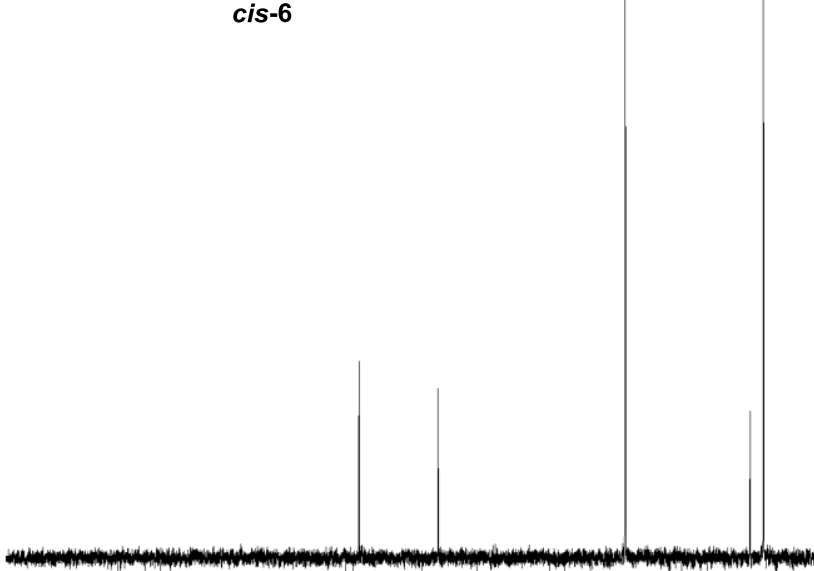

4

(ㅇ.) 웅

ลู่

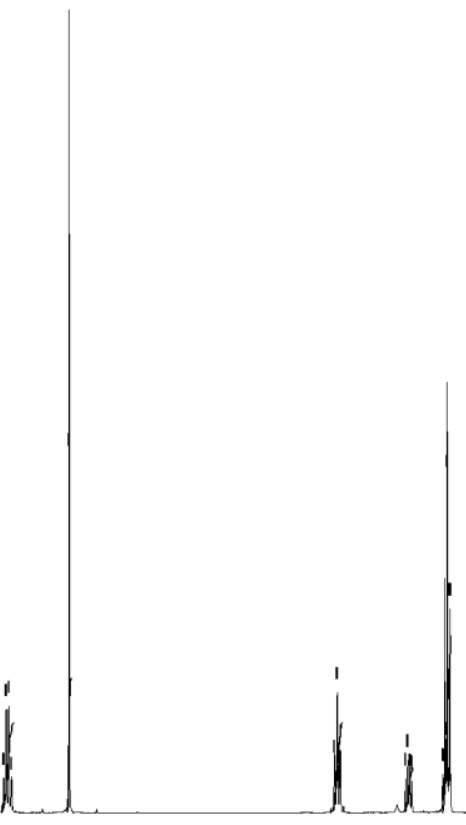

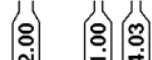
1 ppm 

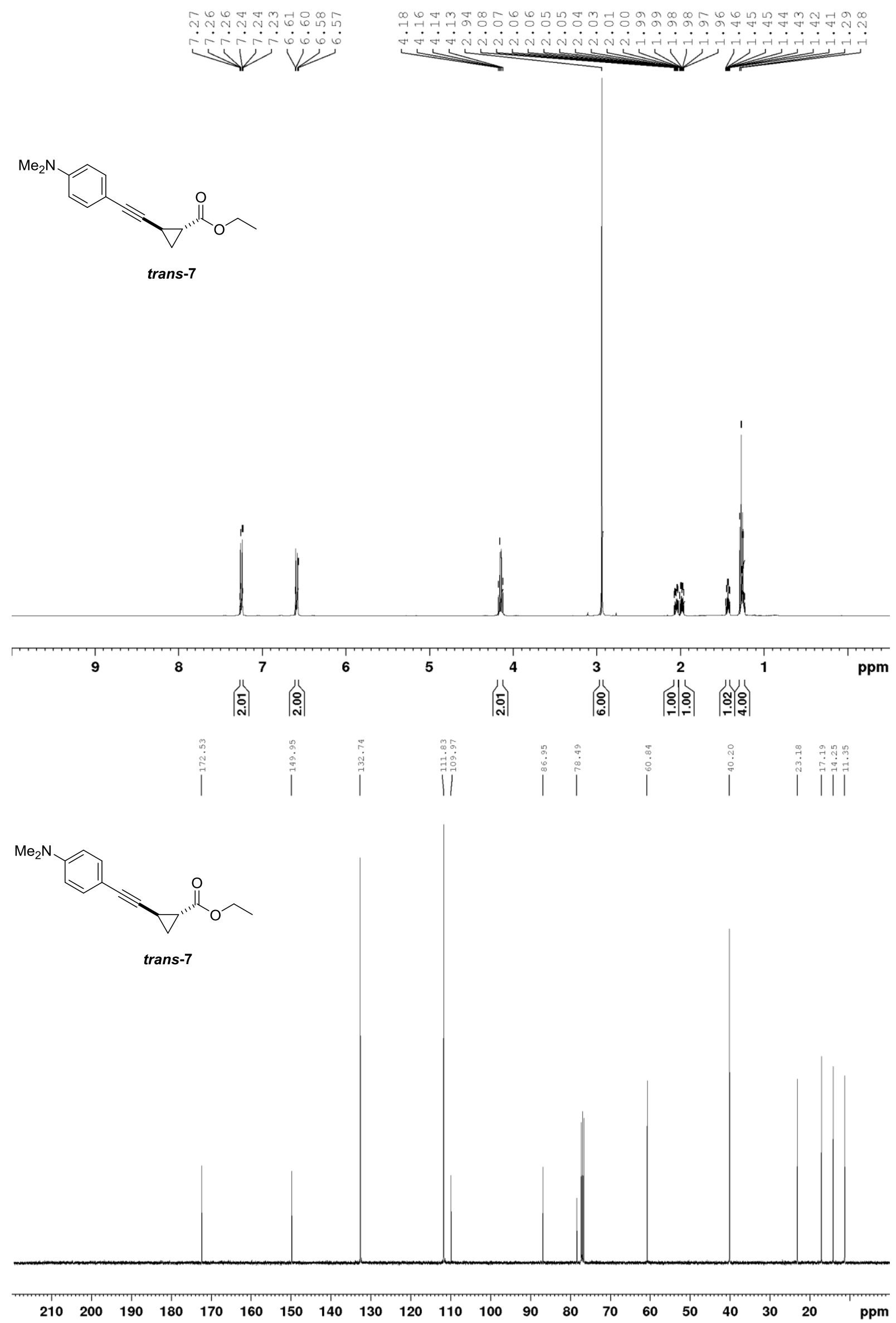

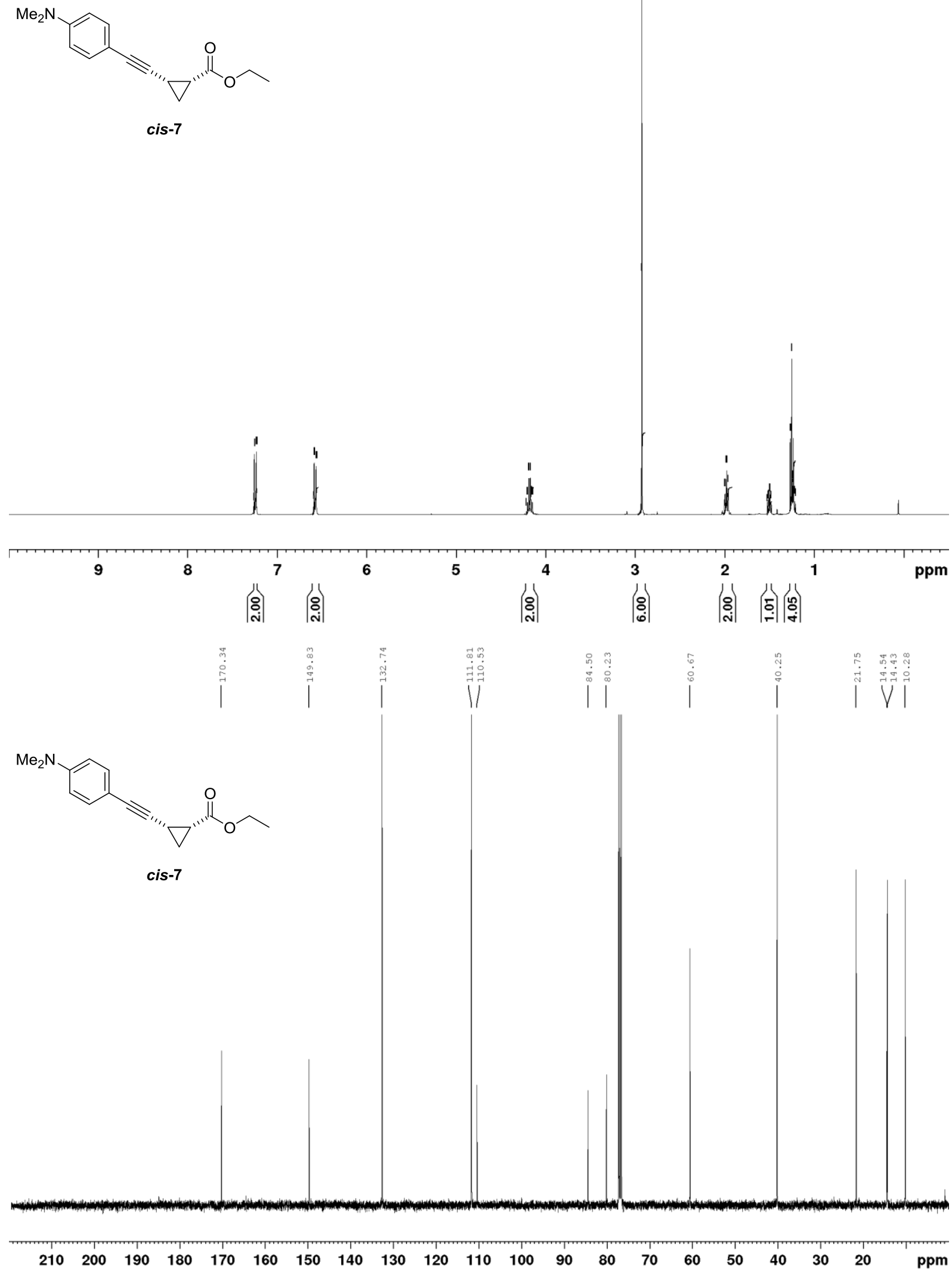


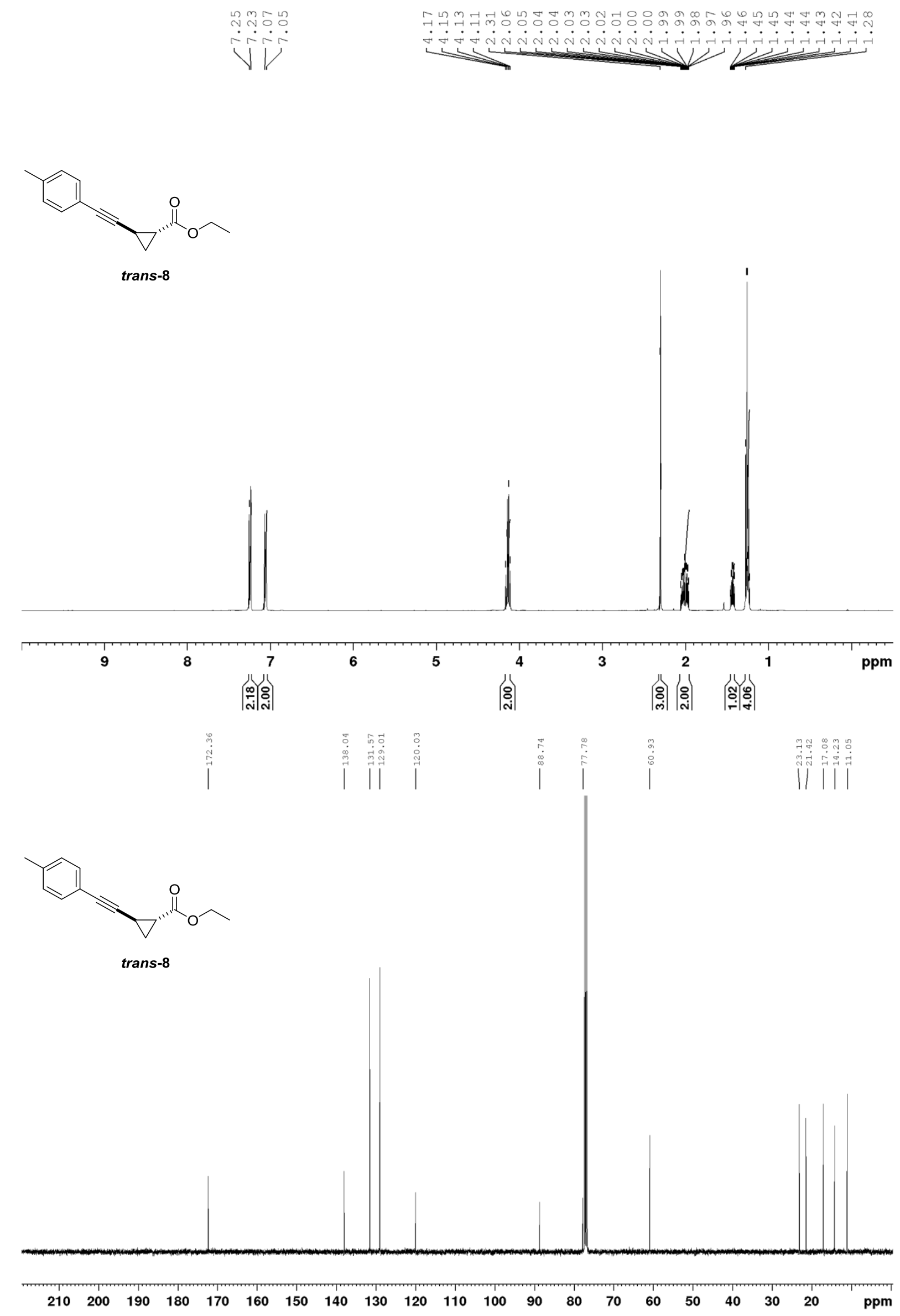




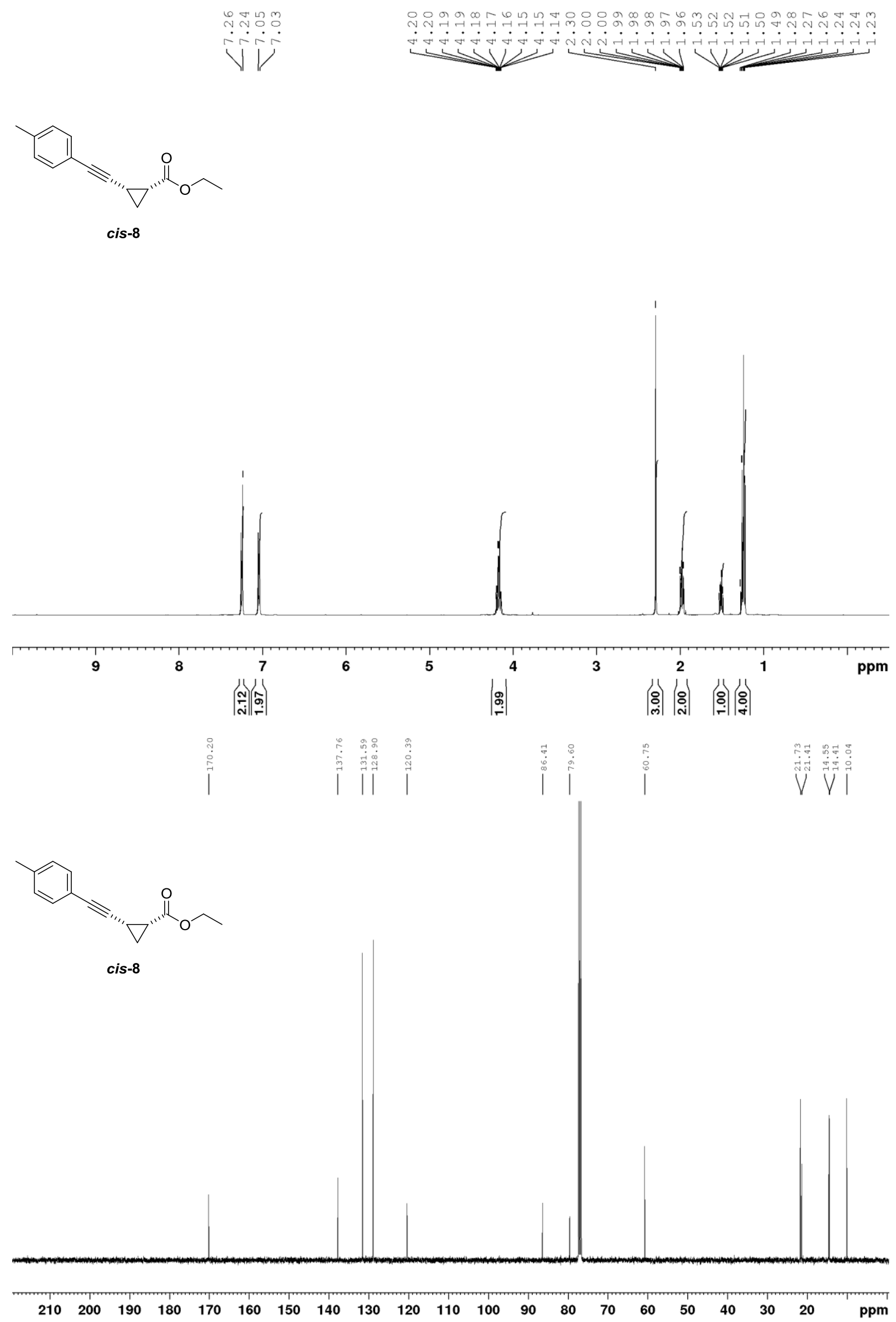




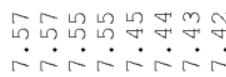

匀供

NC<smiles>CCOC(=O)[C@H]1C[C@H]1c1ccc(I)cc1</smiles>

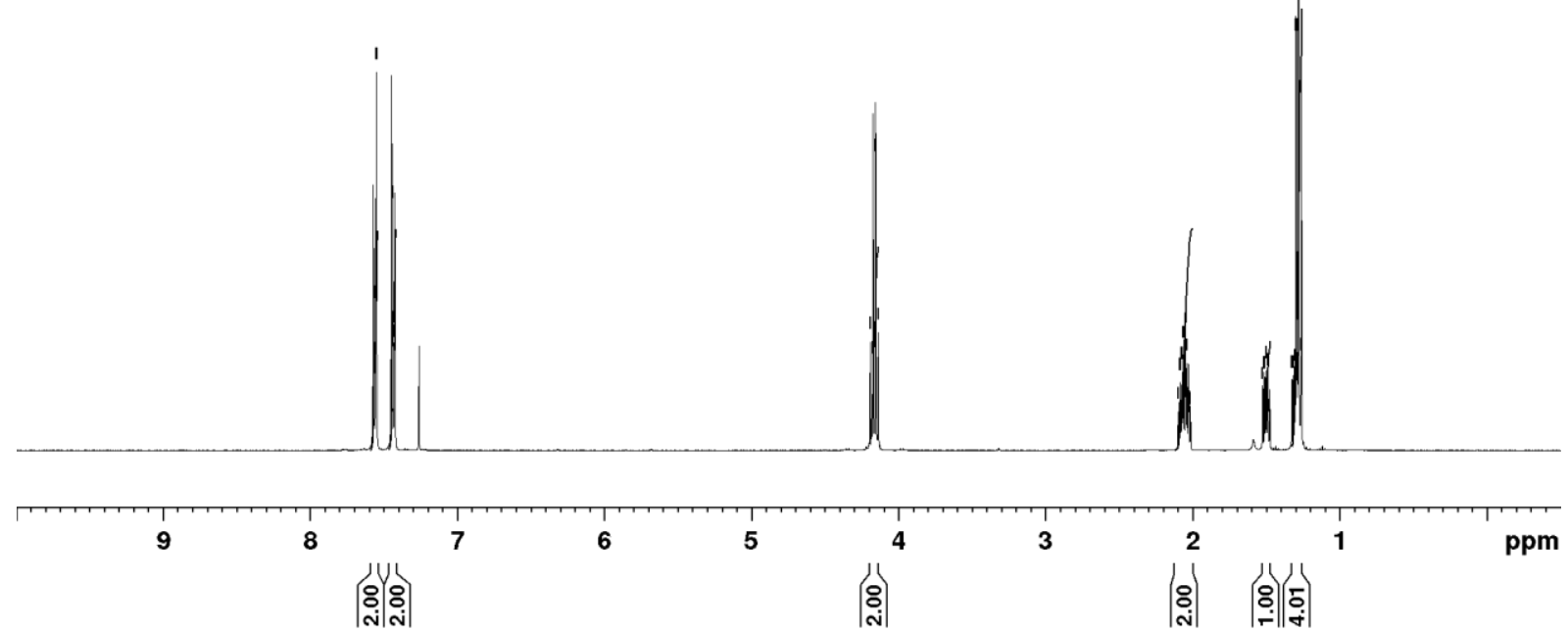

NC

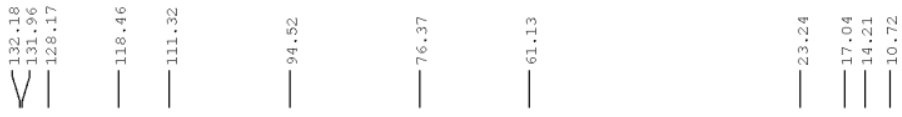<smiles>CCOC(=O)[C@@H]1CC1c1ccccc1</smiles>

trans-9
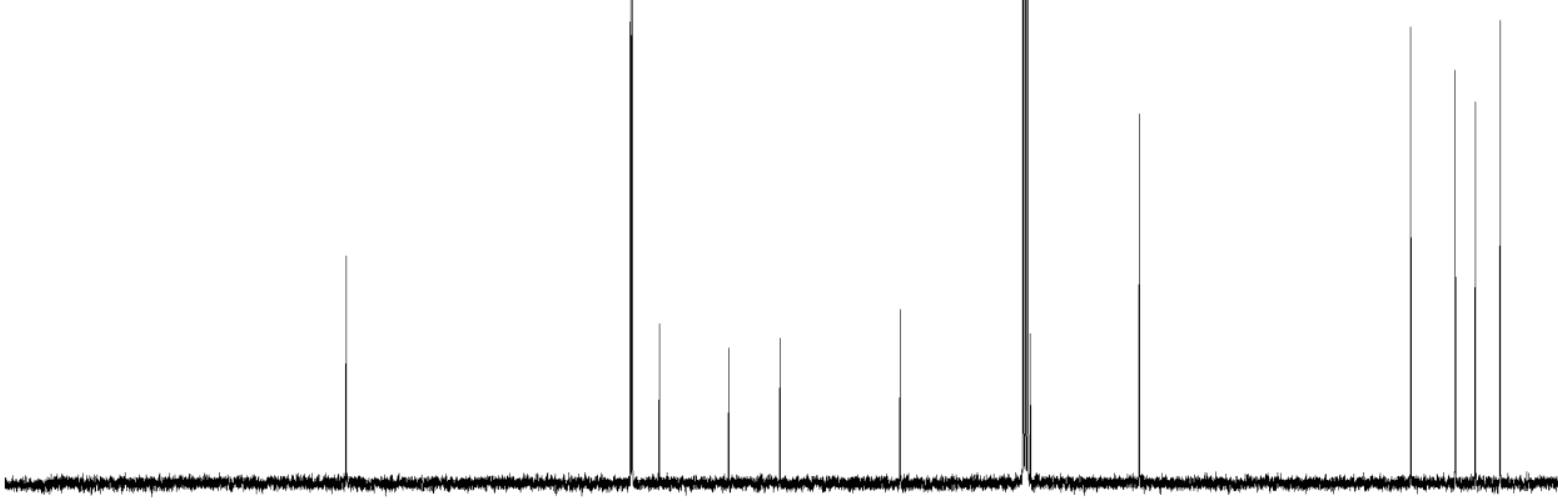
<smiles>CCOC(=O)[C@@H]1C[C@H]1c1ccc(C#N)cc1</smiles>
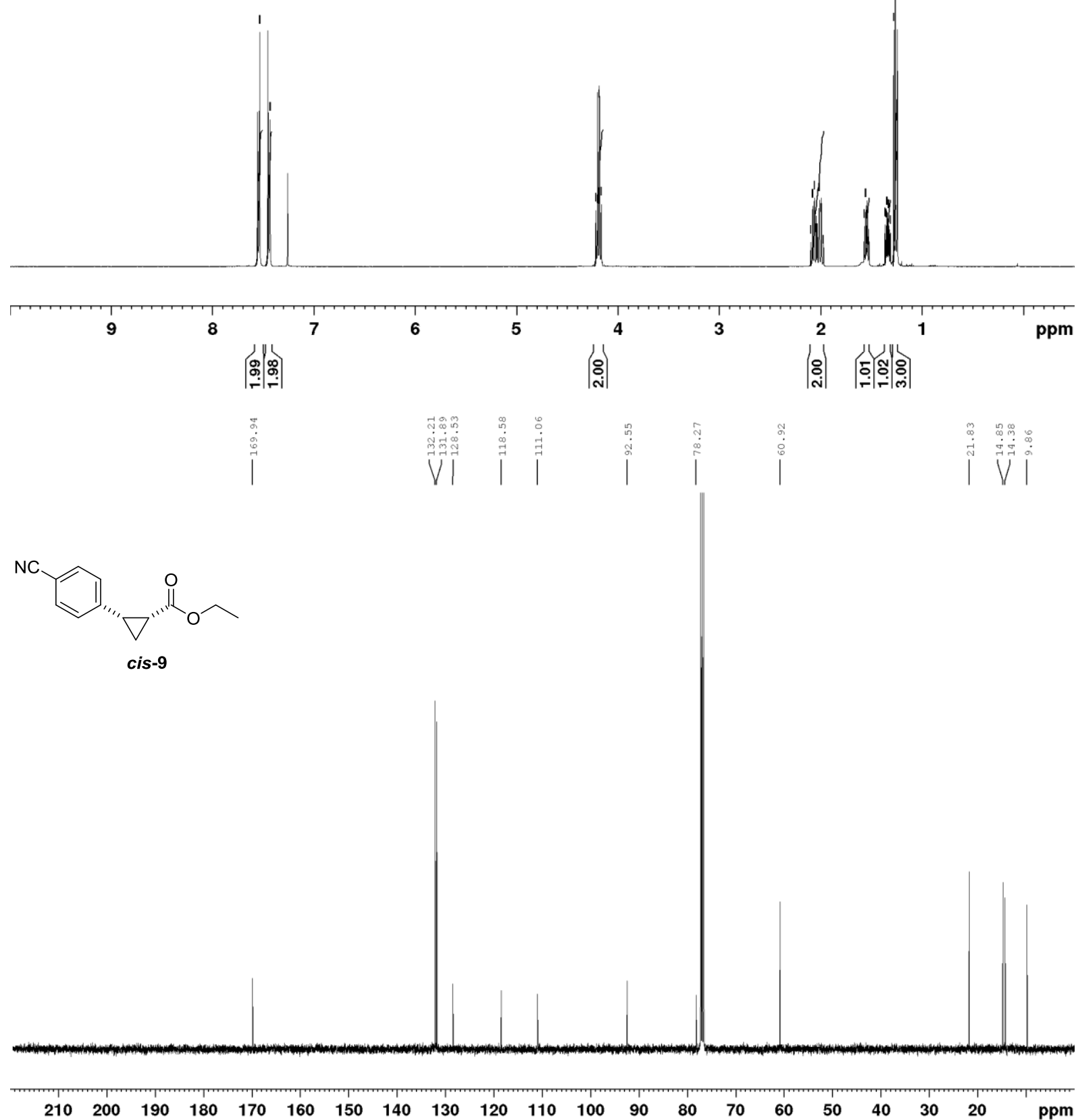


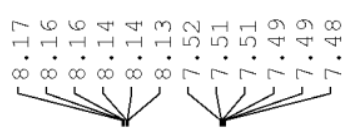
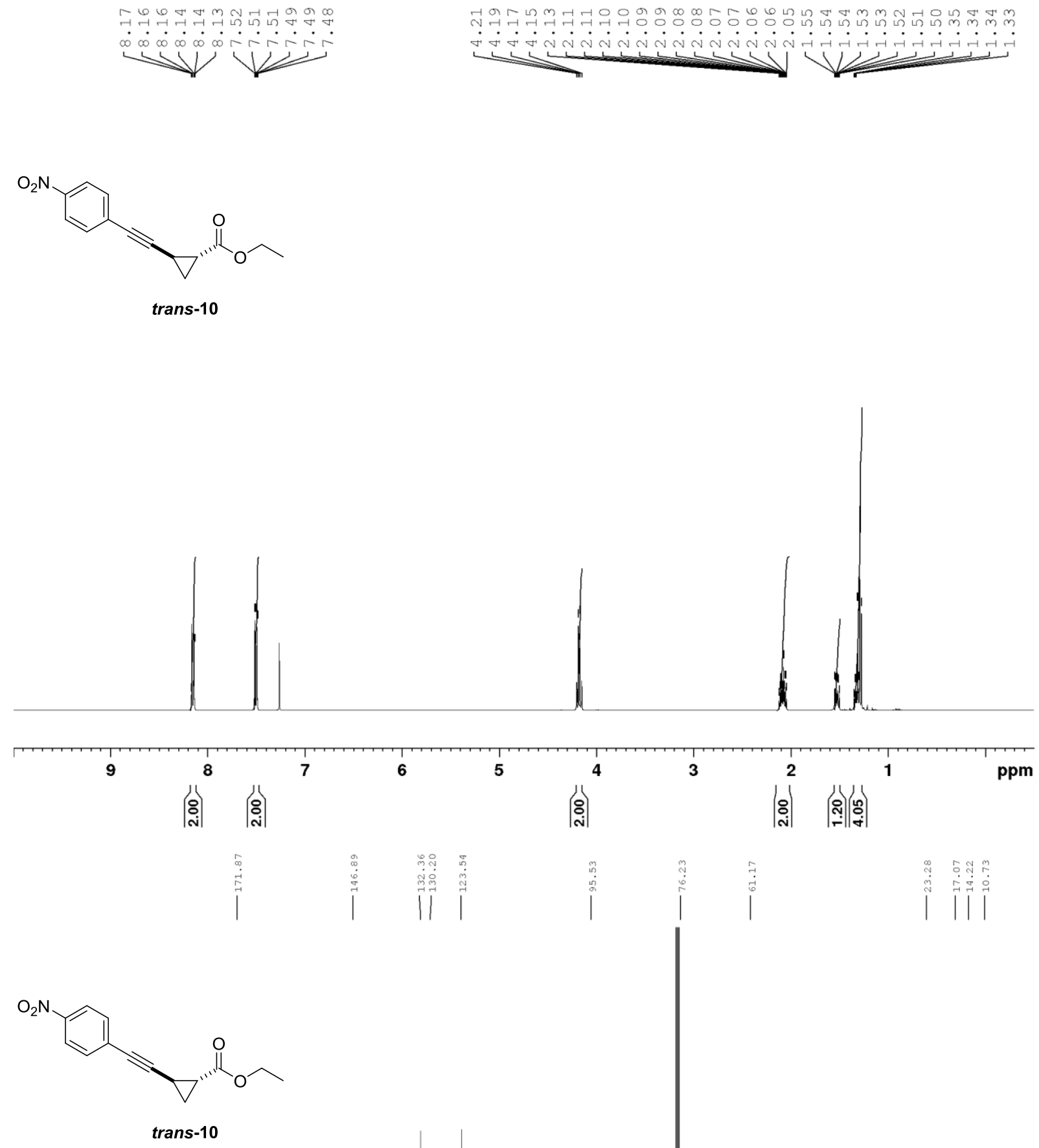

S87 

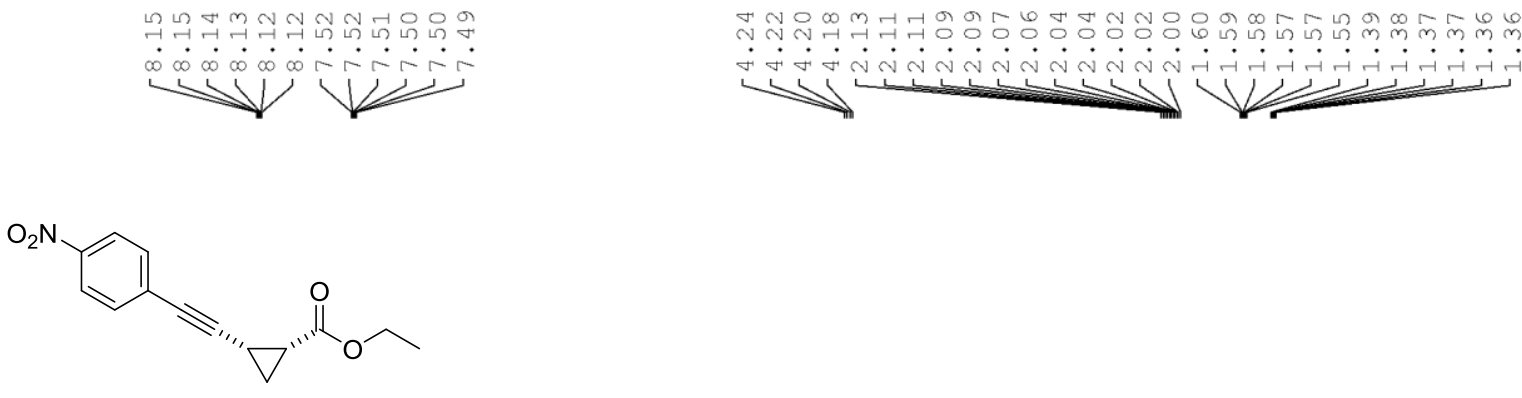

cis-10
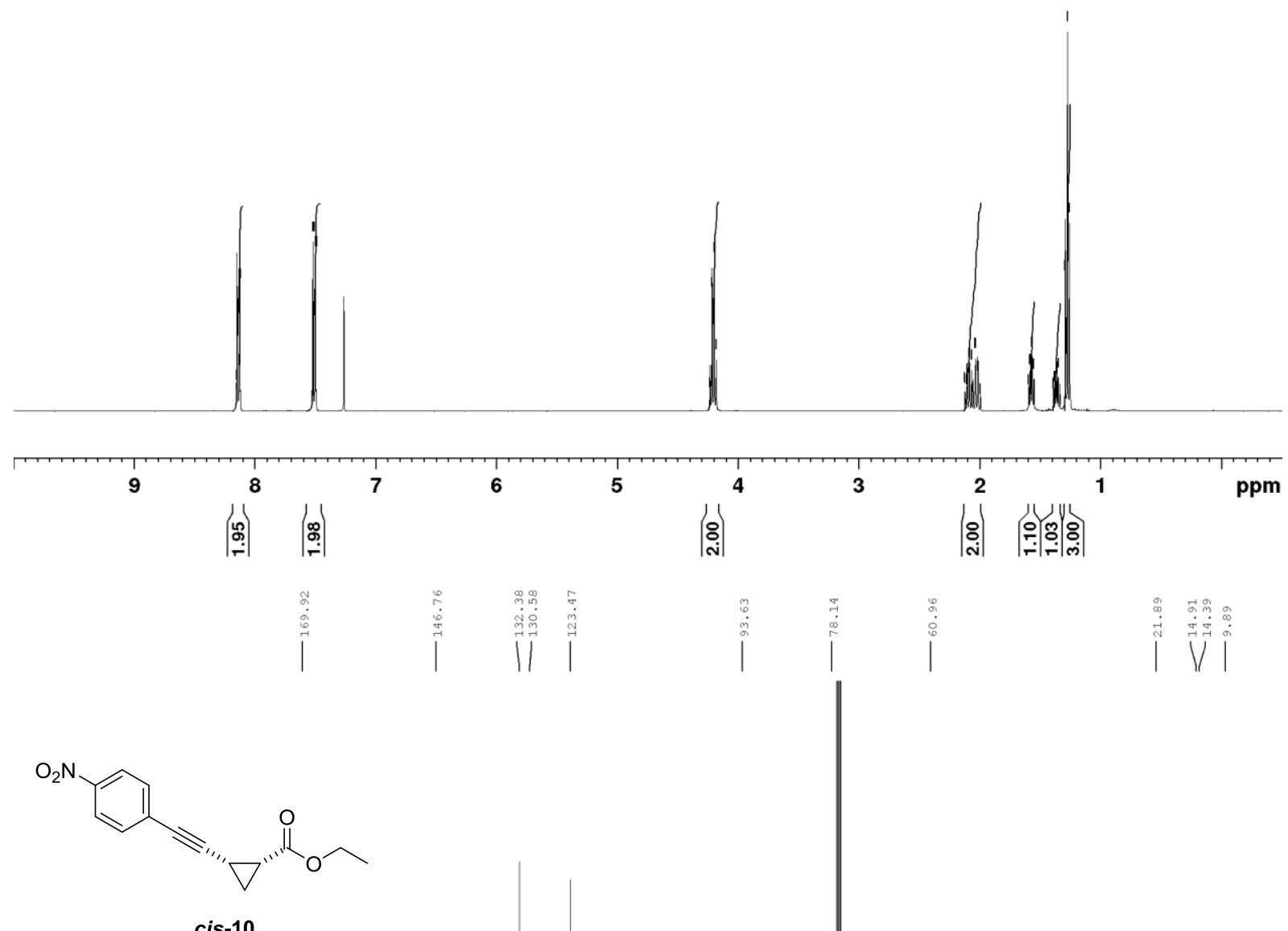

cis -10

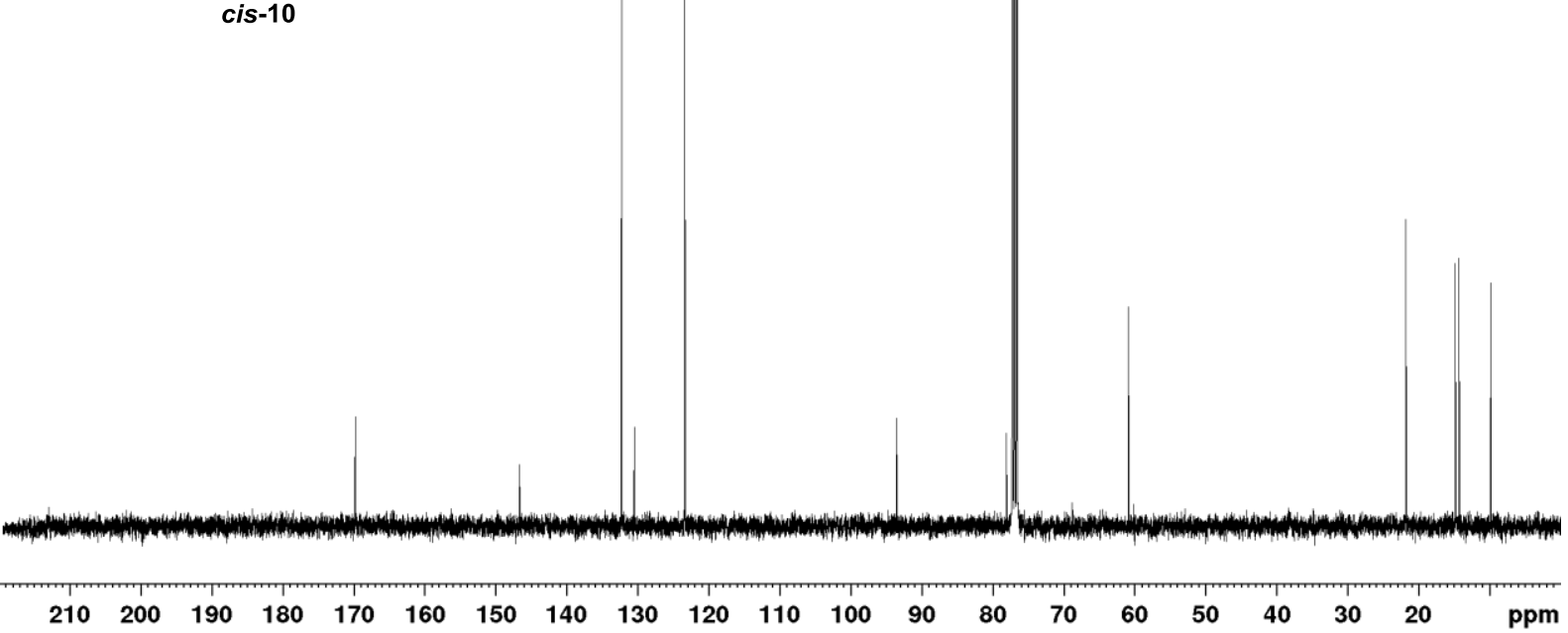




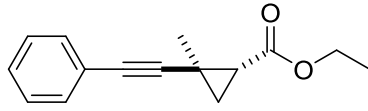

trans-11

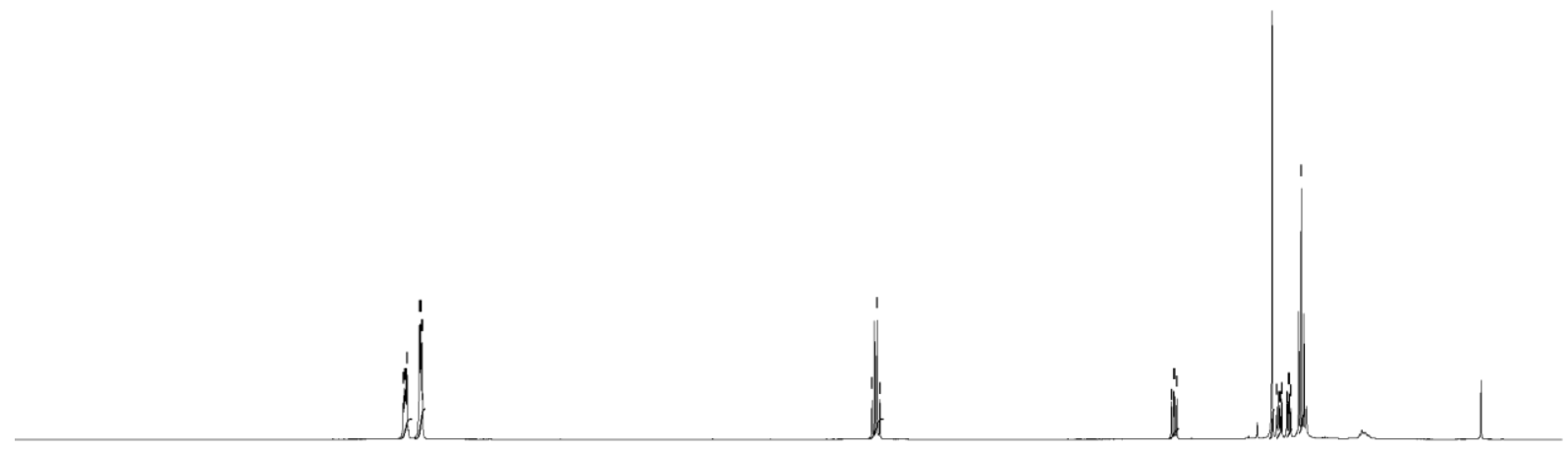

$\begin{array}{llllllllllllllllllll}9.5 & 9.0 & 8.5 & 8.0 & 7.5 & 7.0 & 6.5 & 6.0 & 5.5 & 5.0 & 4.5 & 4.0 & 3.5 & 3.0 & 2.5 & 2.0 & 1.5 & 1.0 & 0.5 & \mathrm{ppm}\end{array}$

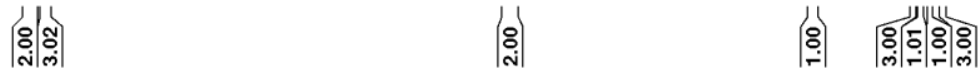
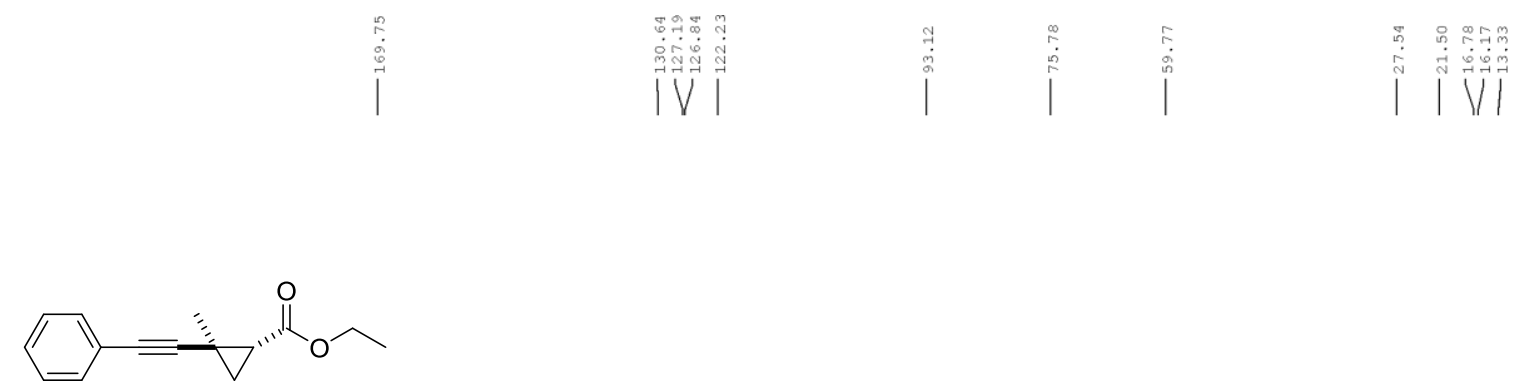

trans-11

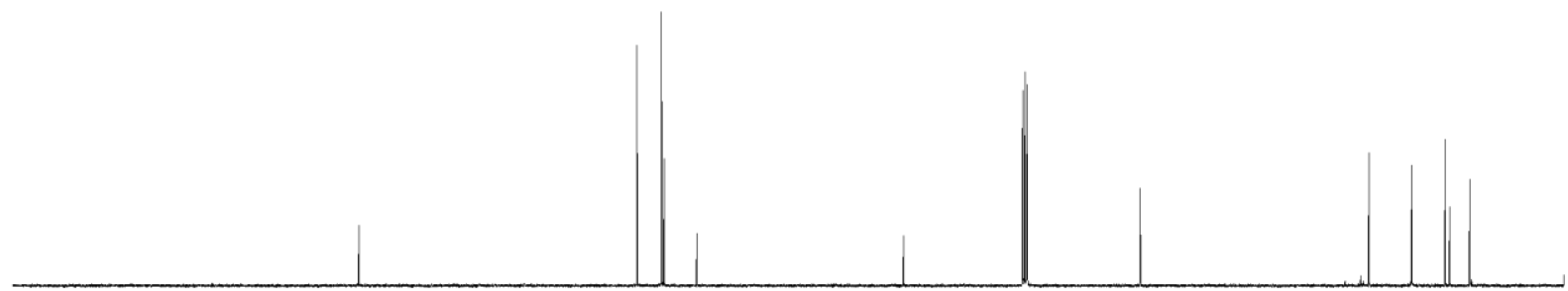

$\begin{array}{llllllllllllllllllllll}210 & 200 & 190 & 180 & 170 & 160 & 150 & 140 & 130 & 120 & 110 & 100 & 90 & 80 & 70 & 60 & 50 & 40 & 30 & 20 & \mathrm{ppm}\end{array}$ 


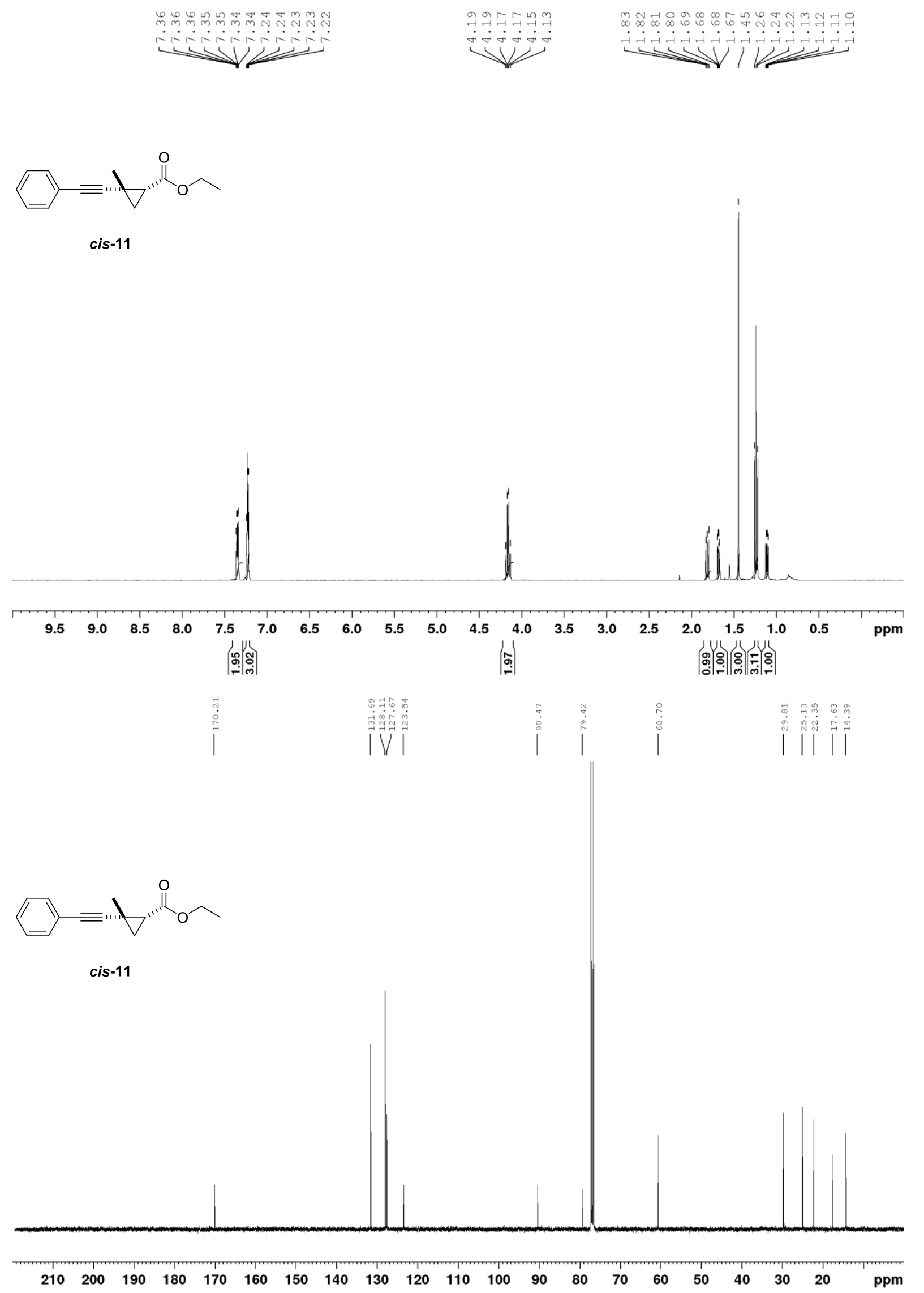




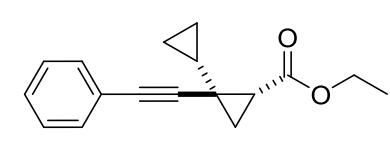

trans-12
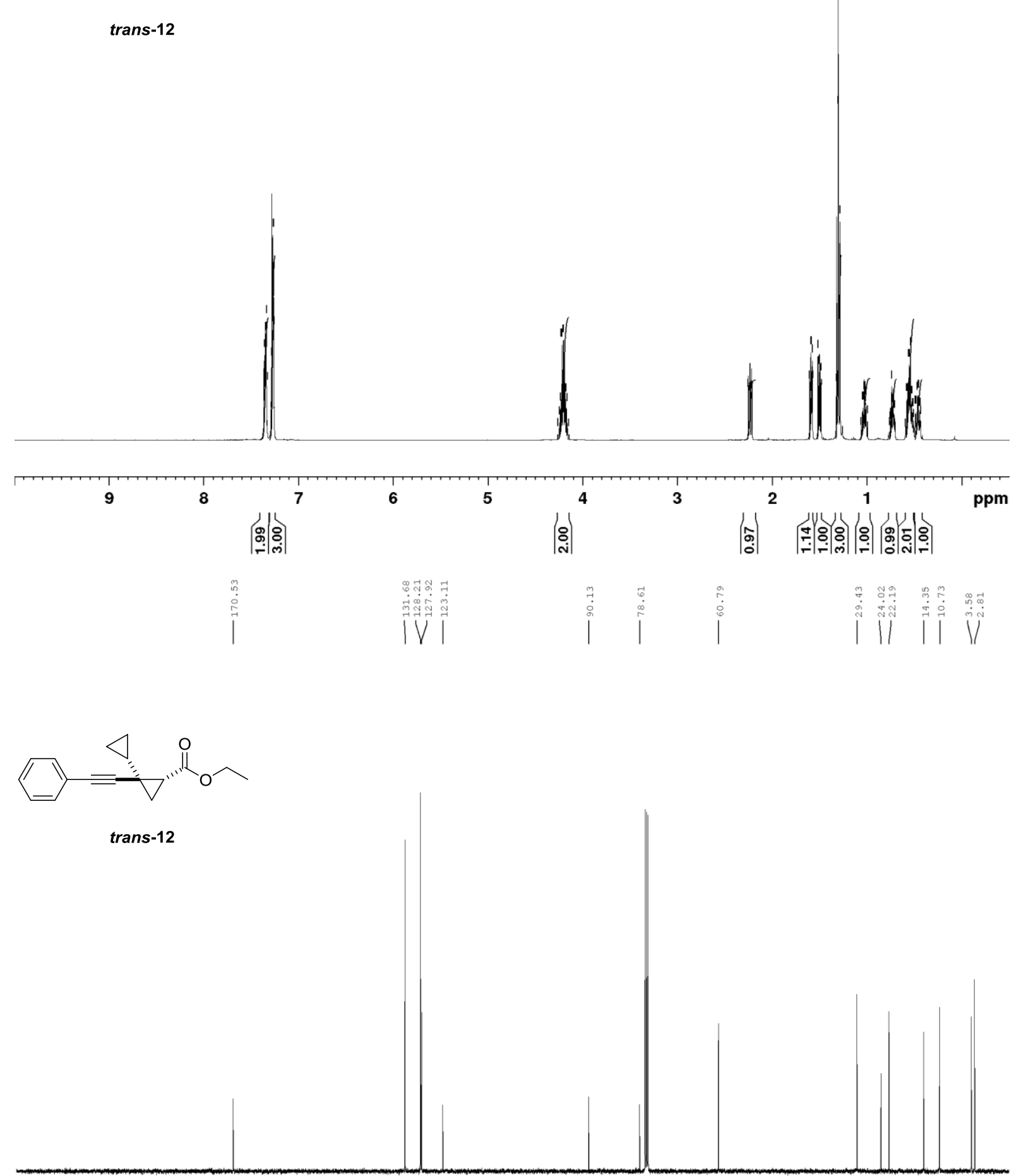

$\begin{array}{lllllllllllllllllllllll}210 & 200 & 190 & 180 & 170 & 160 & 150 & 140 & 130 & 120 & 110 & 100 & 90 & 80 & 70 & 60 & 50 & 40 & 30 & 20 & 10 & \text { ppm }\end{array}$ 


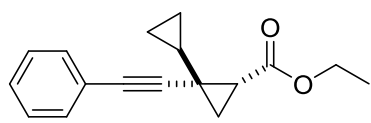

cis-12
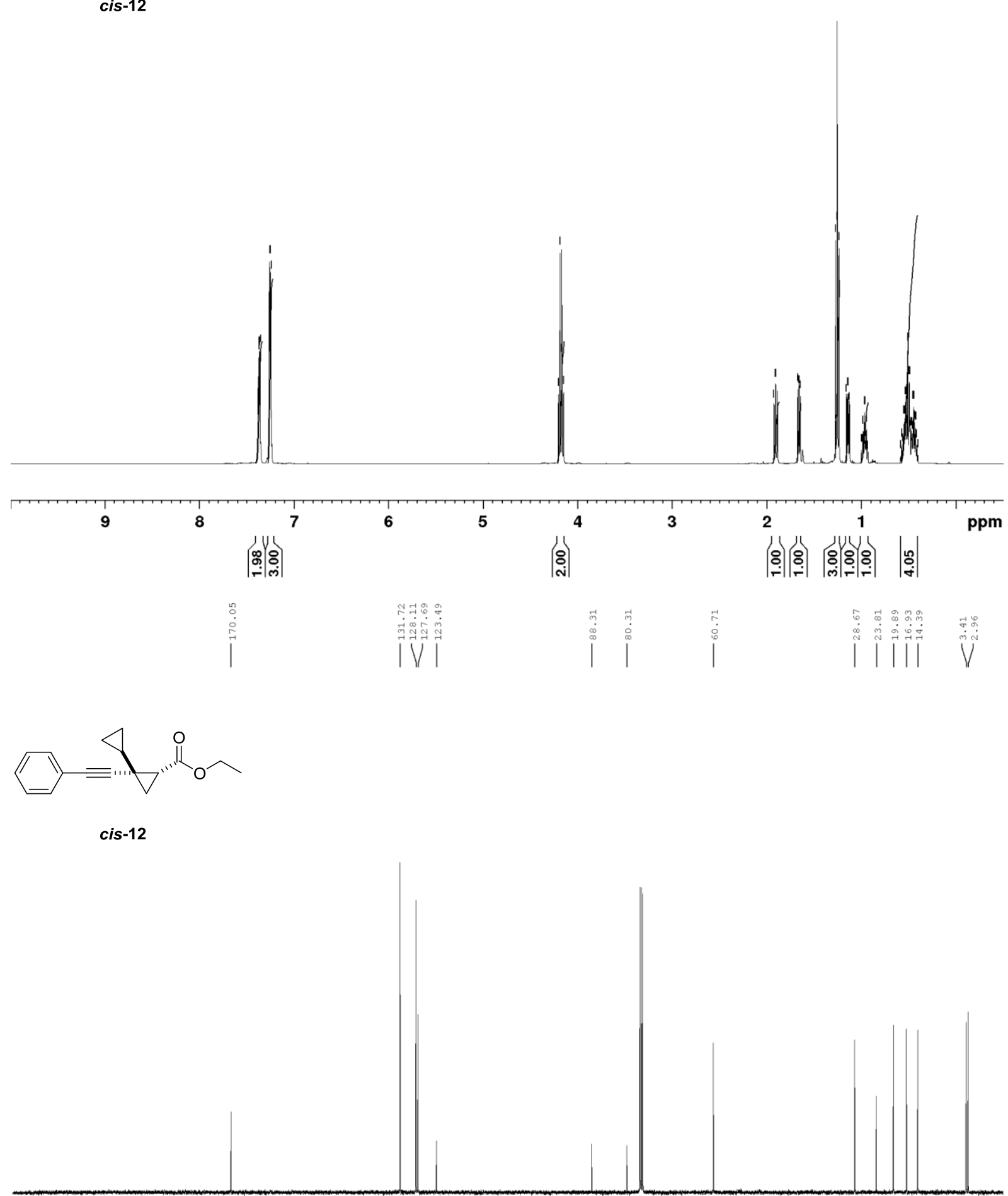

$\begin{array}{lllllllllllllllllllllllllll}210 & 200 & 190 & 180 & 170 & 160 & 150 & 140 & 130 & 120 & 110 & 100 & 90 & 80 & 70 & 60 & 50 & 40 & 30 & 20 & 10 & \text { ppm }\end{array}$ 
<smiles>CCOC(=O)[C@H]1CC1C#CC(O)c1ccccc1</smiles>

trans-13
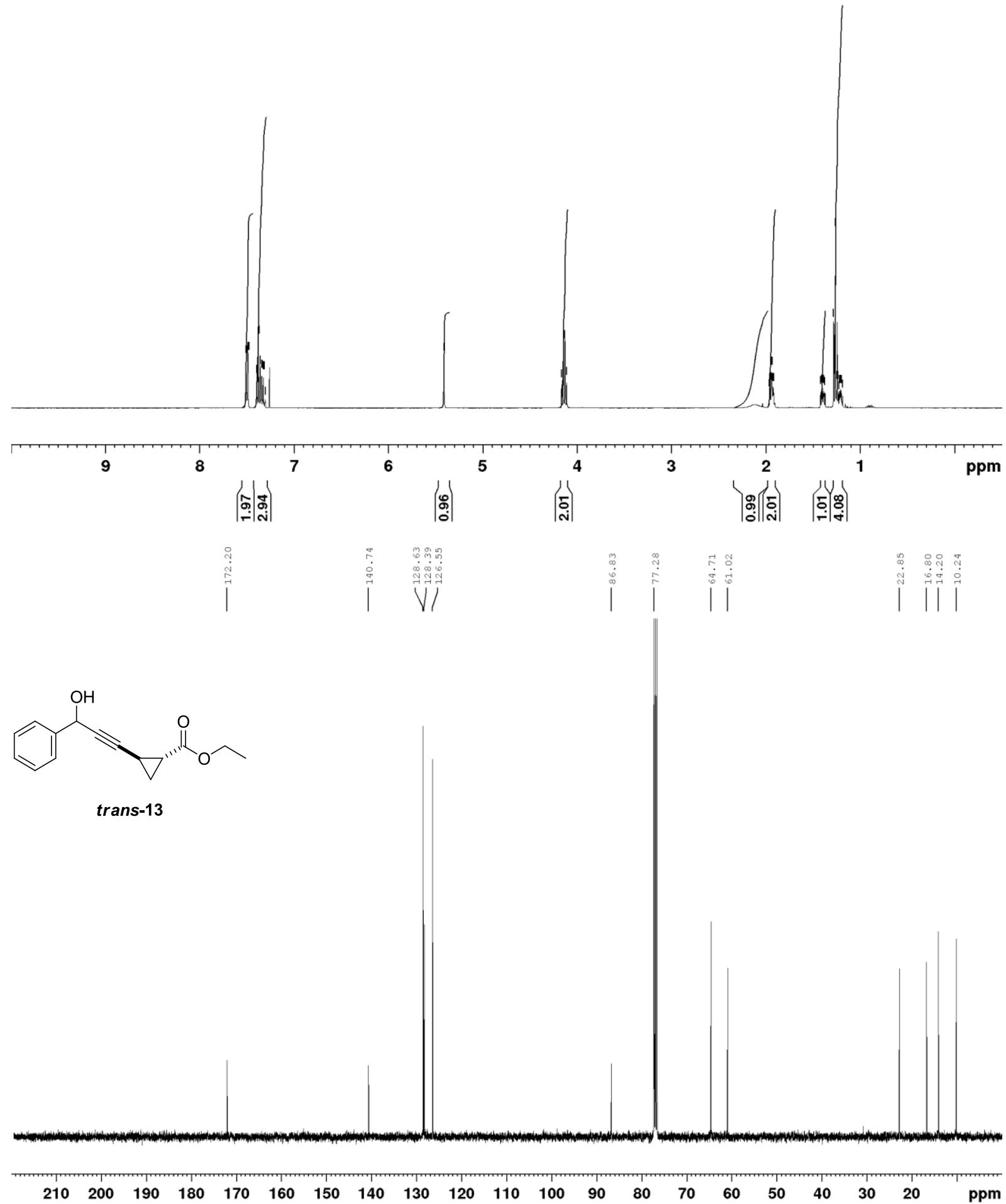
<smiles>CCOC(=O)[C@@H]1C[C@@H]1C#CC(O)c1ccccc1</smiles>
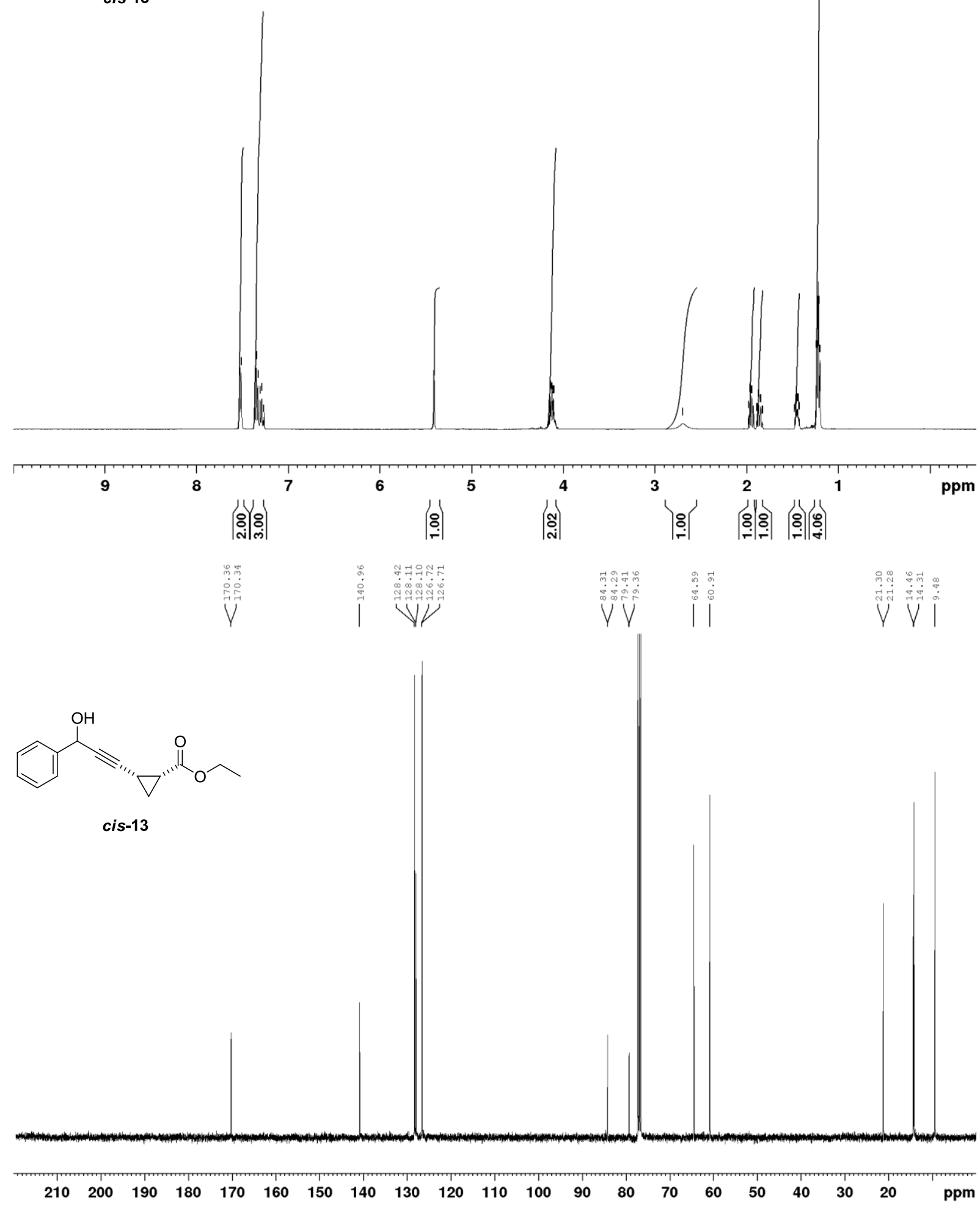

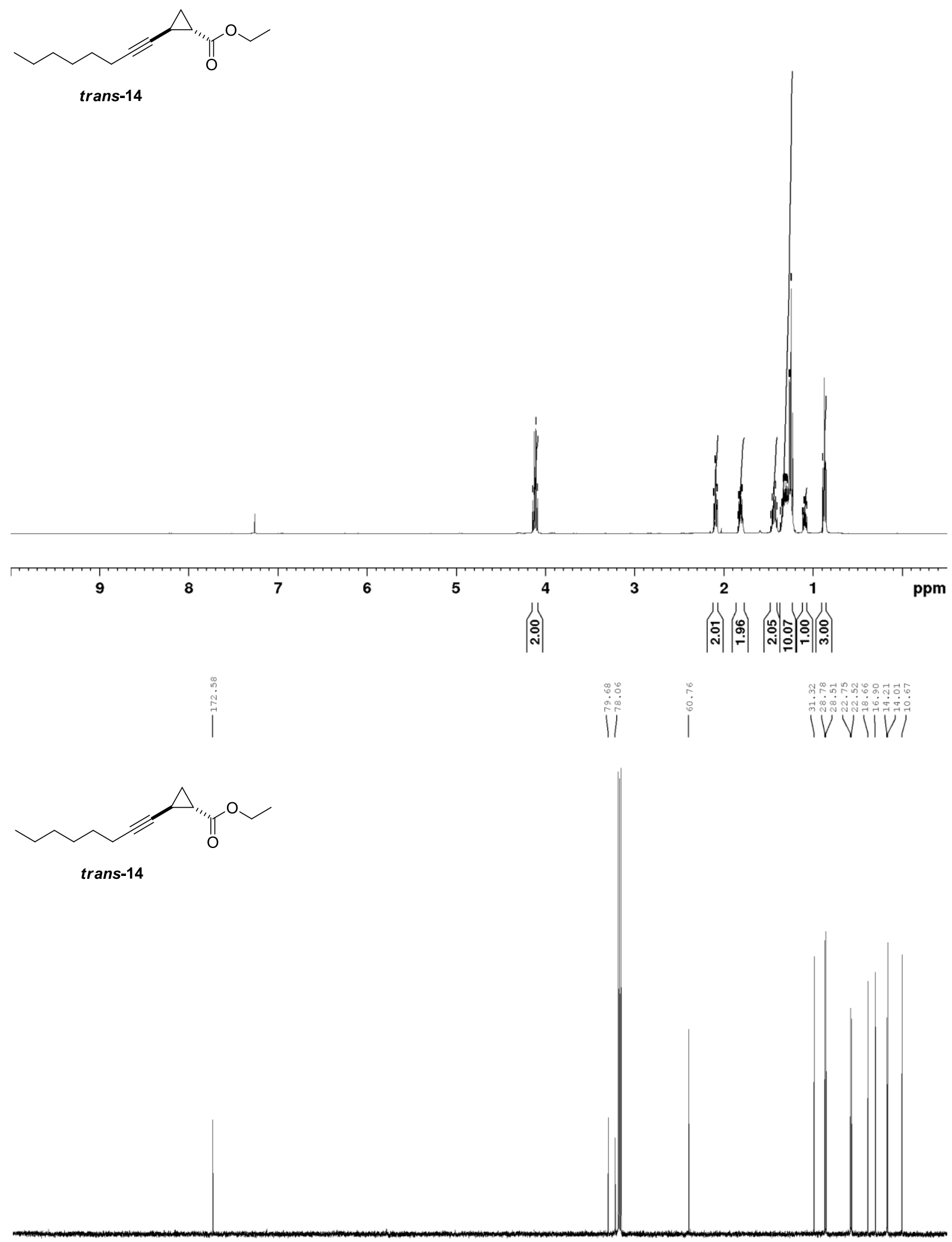

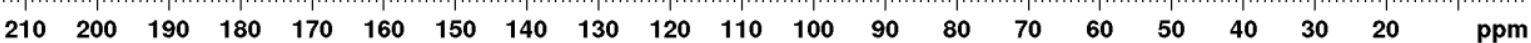



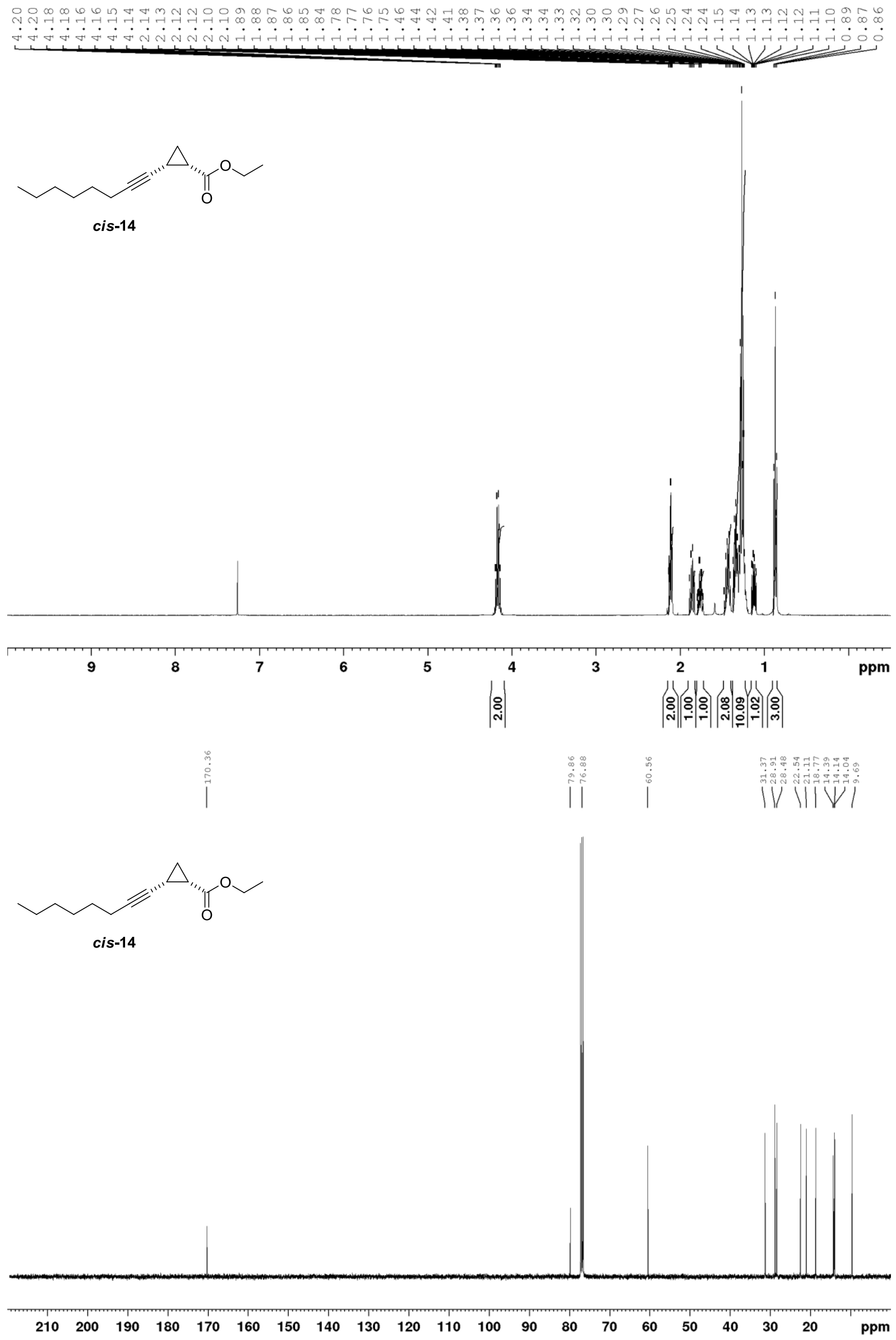

S96 


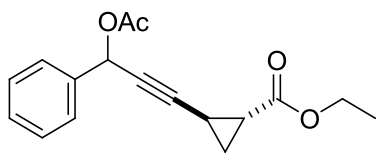

trans-15
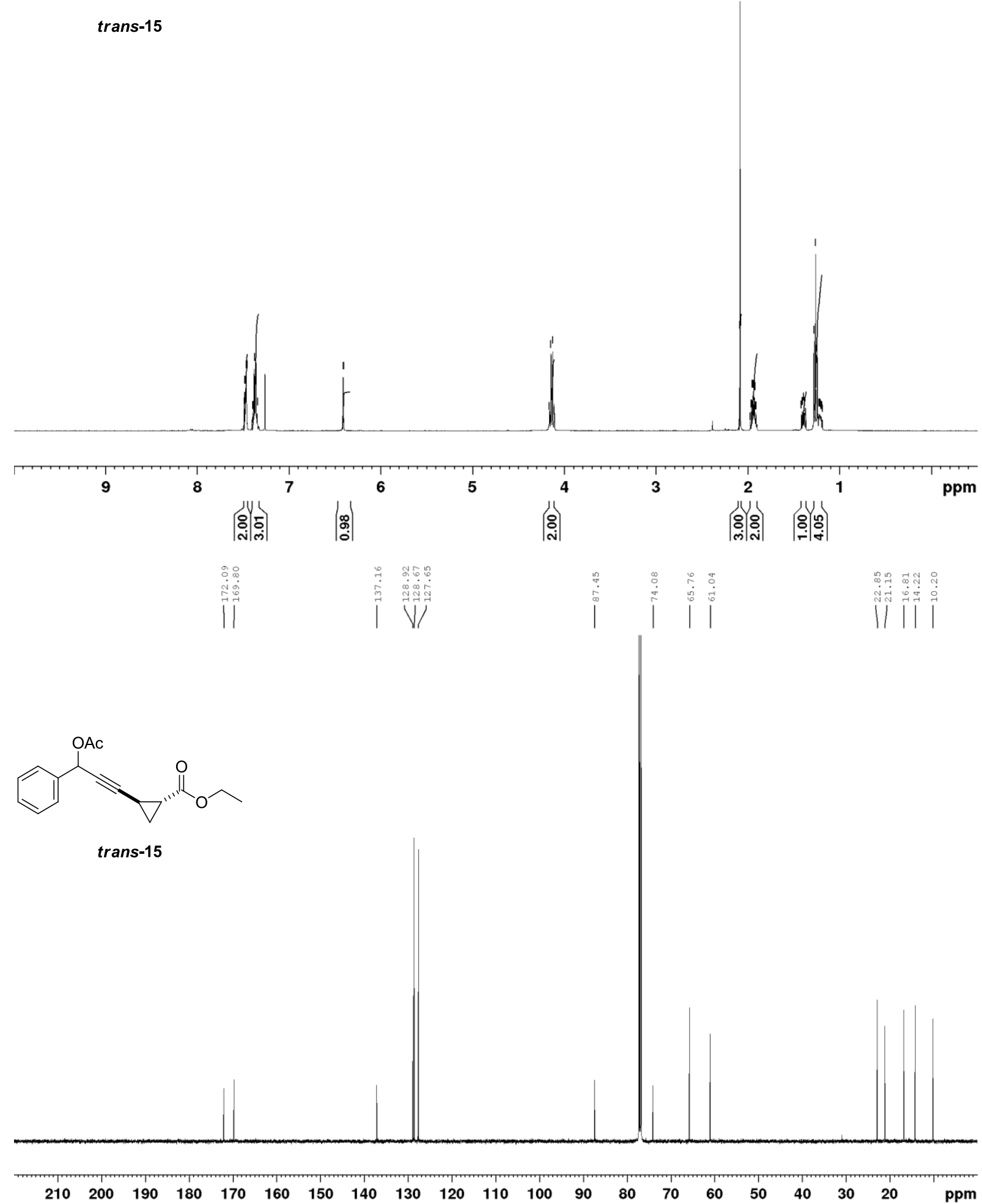

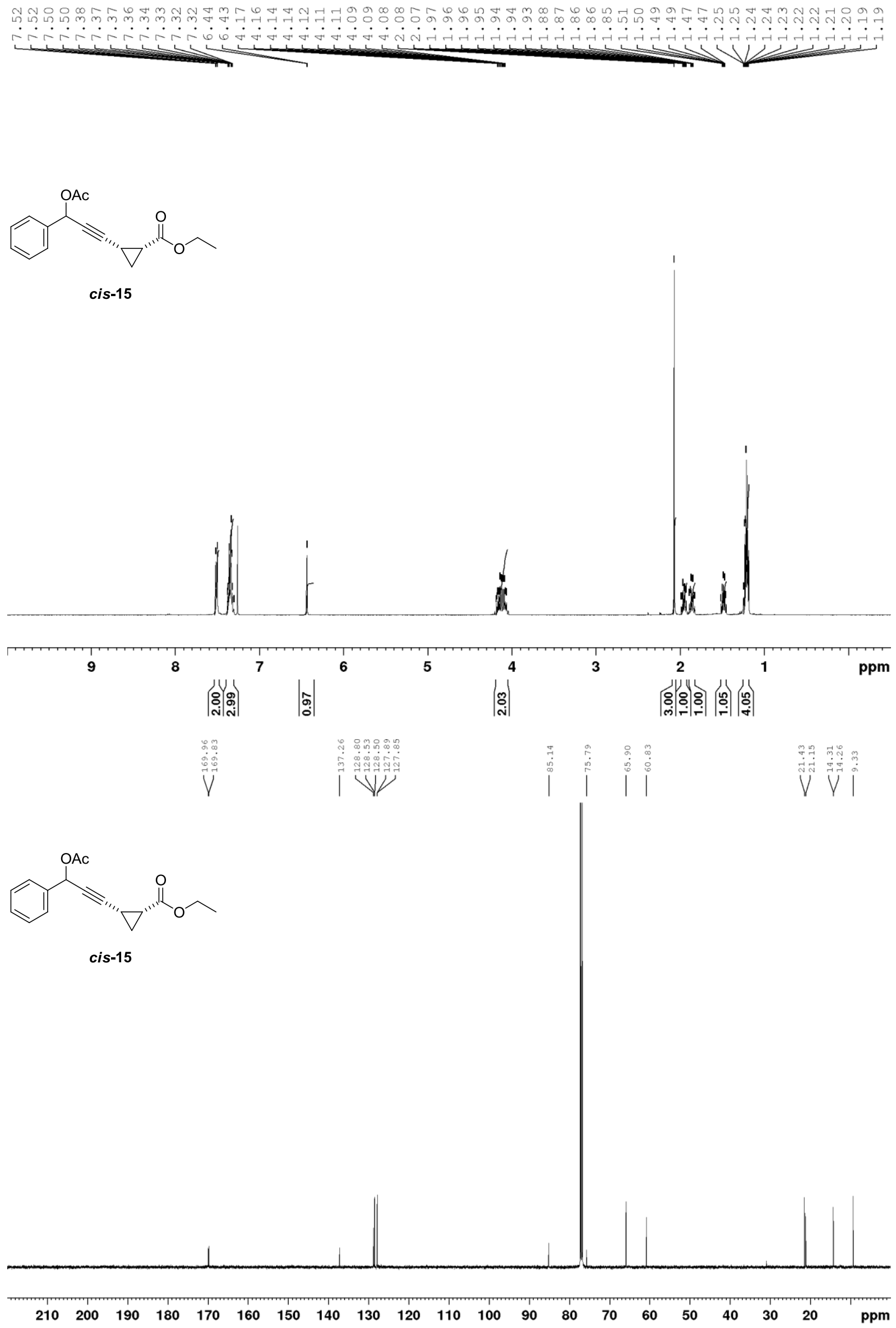

S98 

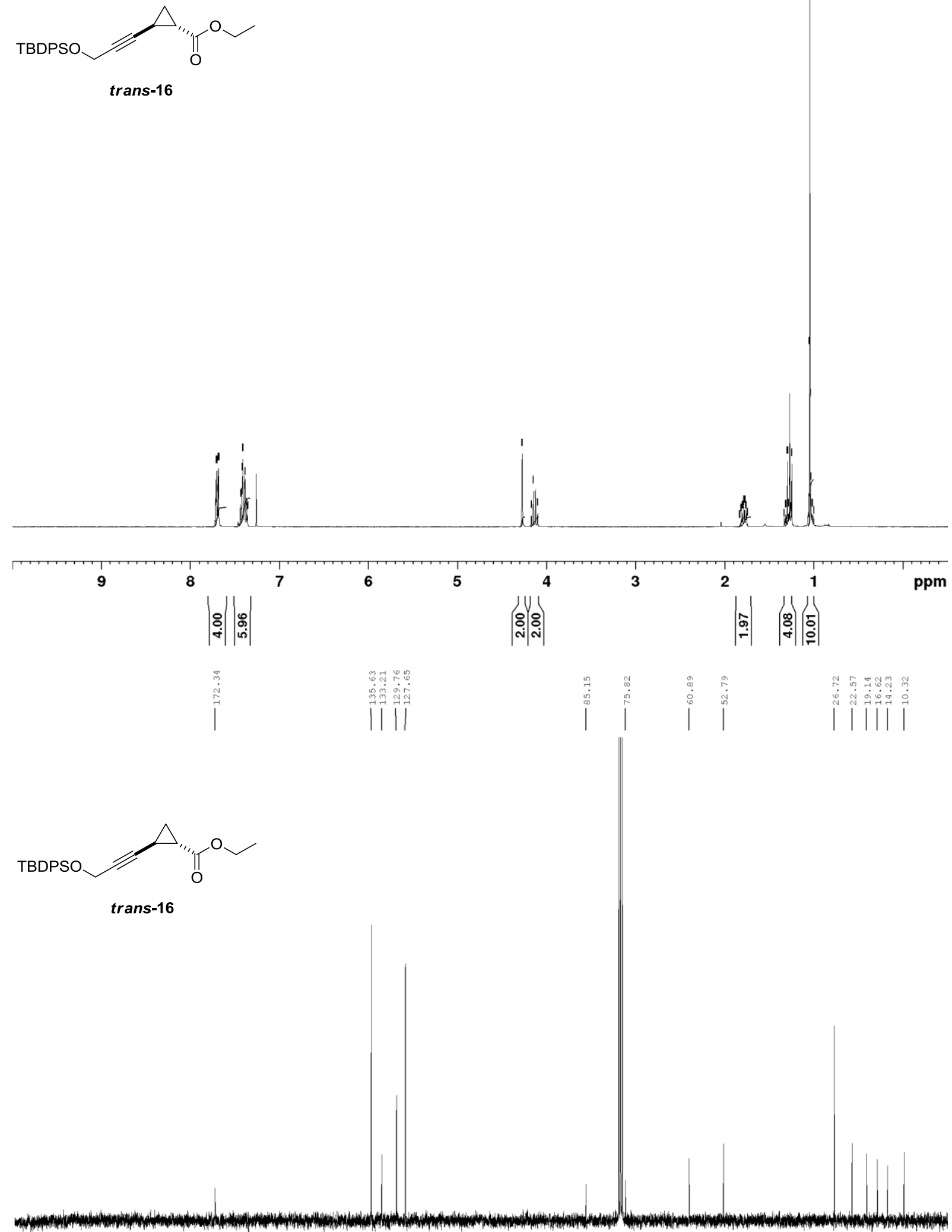

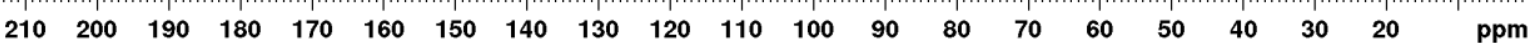


cis-16
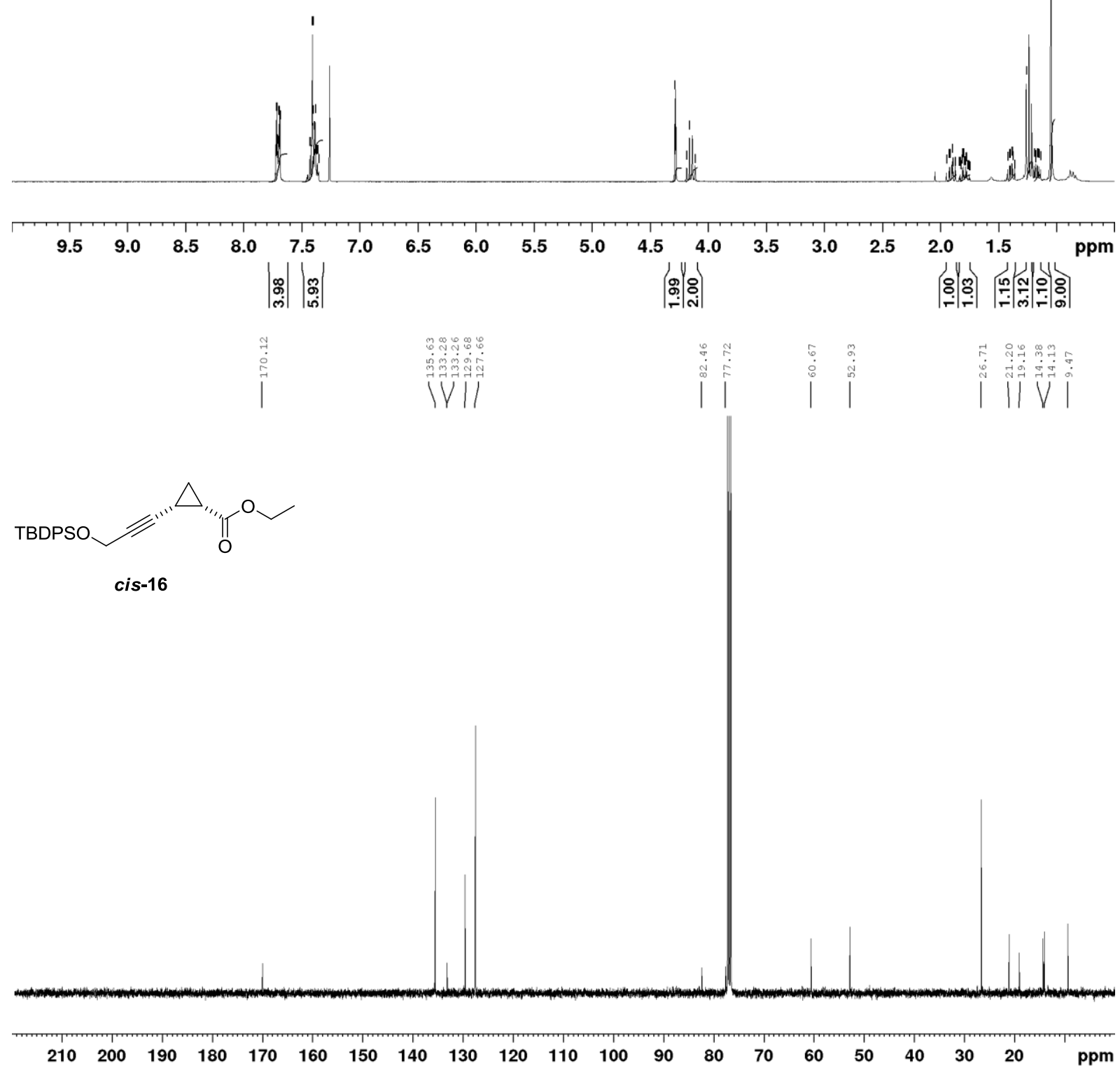

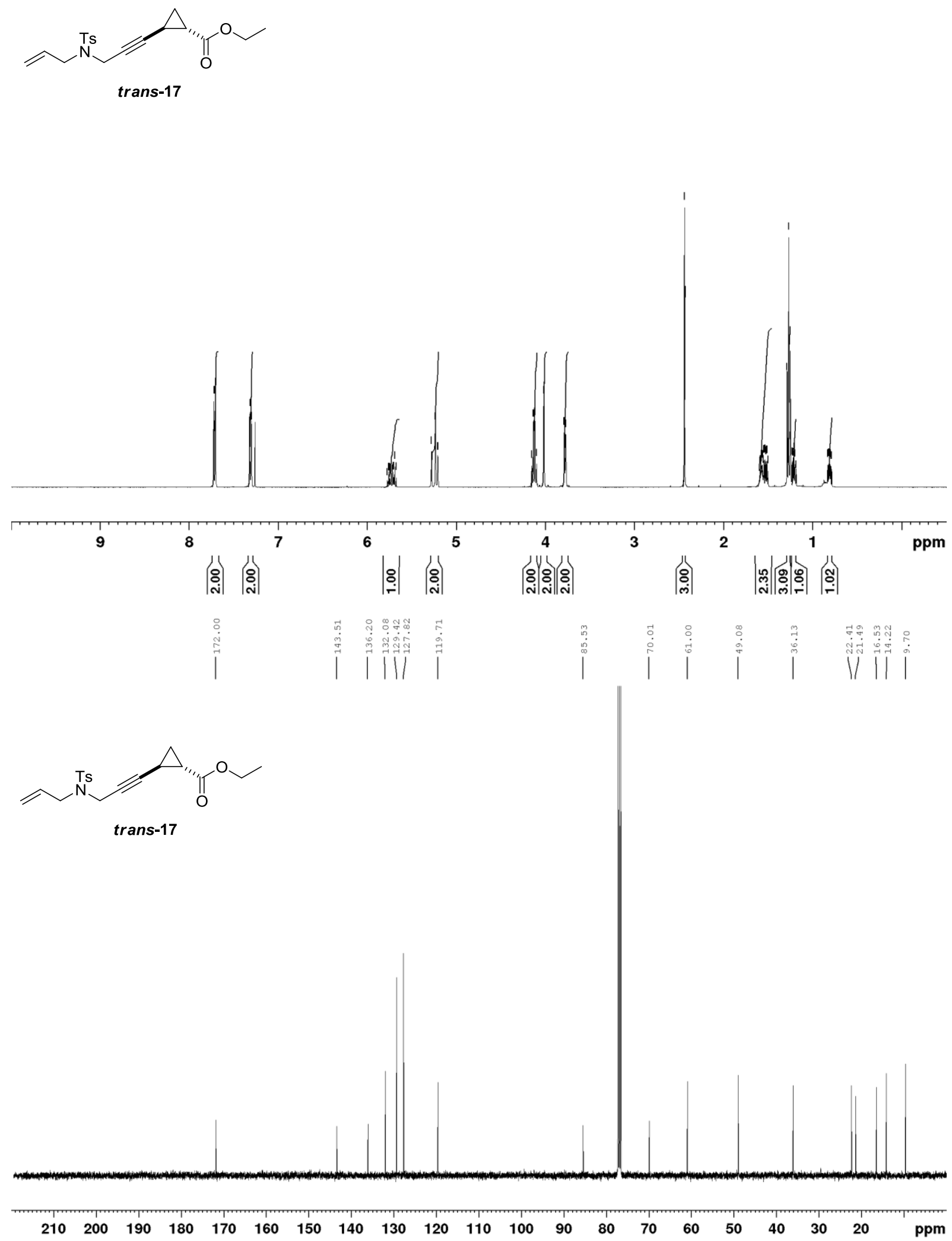

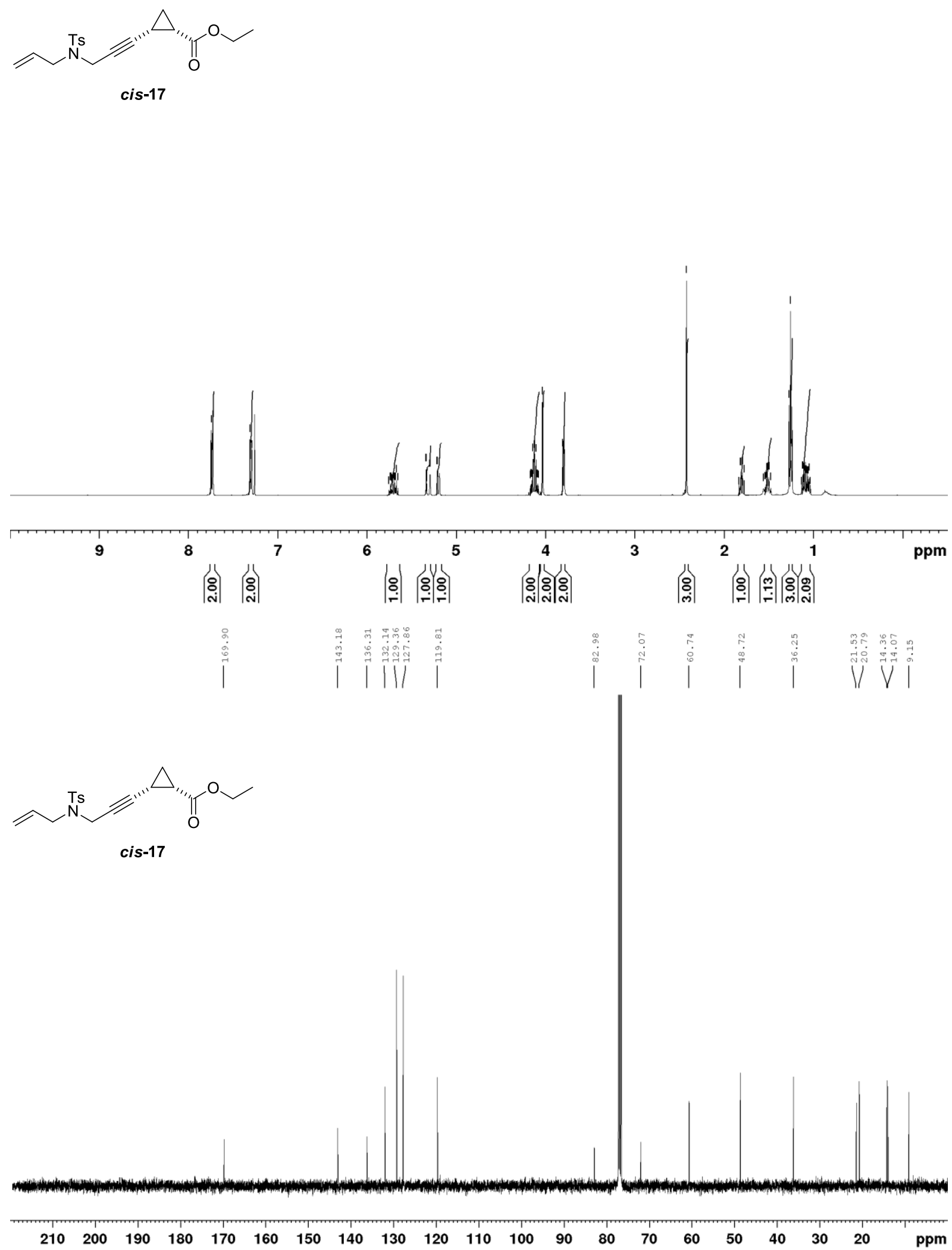
<smiles>CCOC(=O)[C@@H]1CC1C#CCN[AsH2]</smiles>
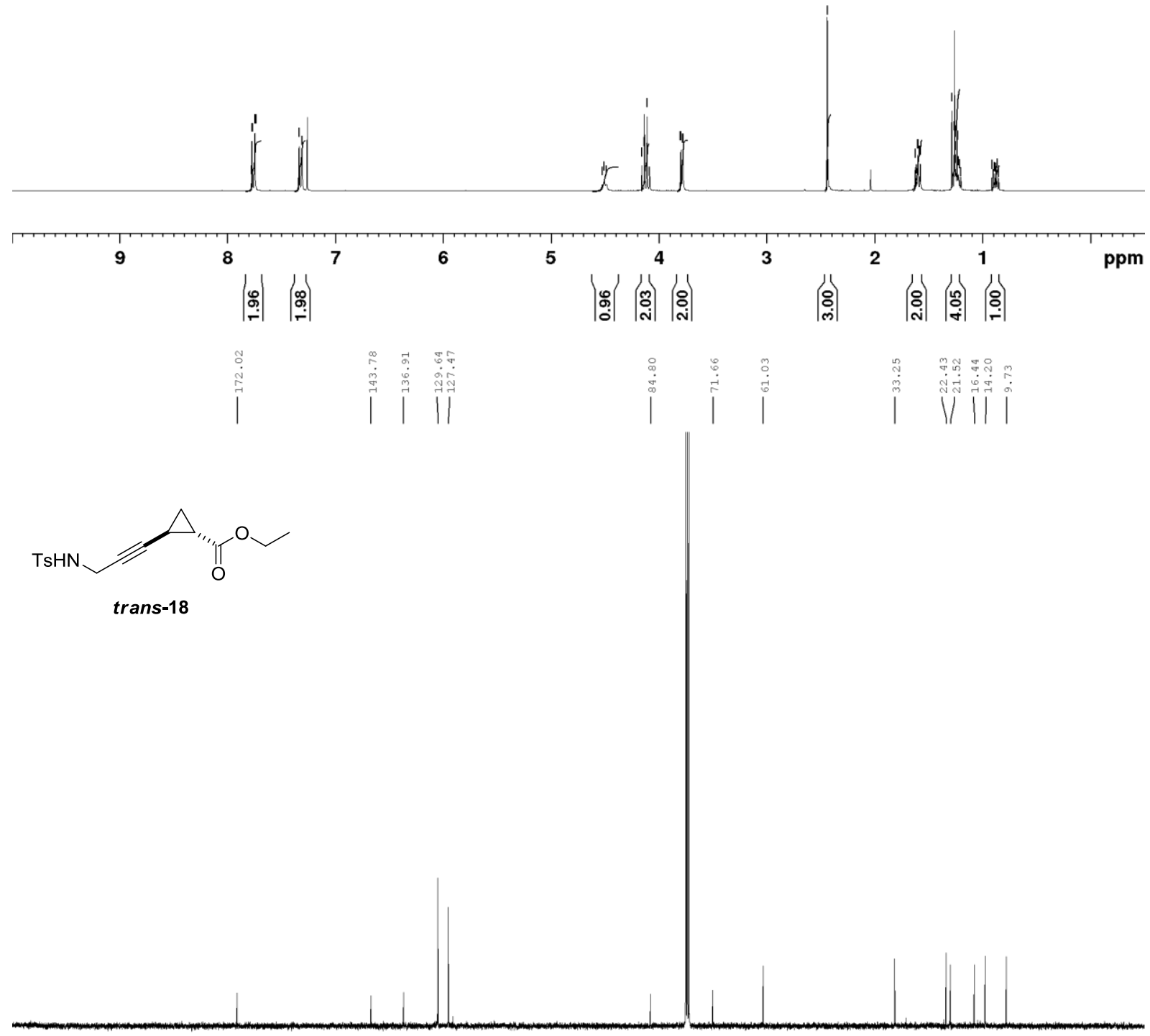

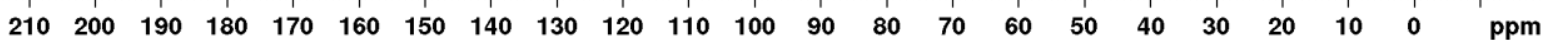



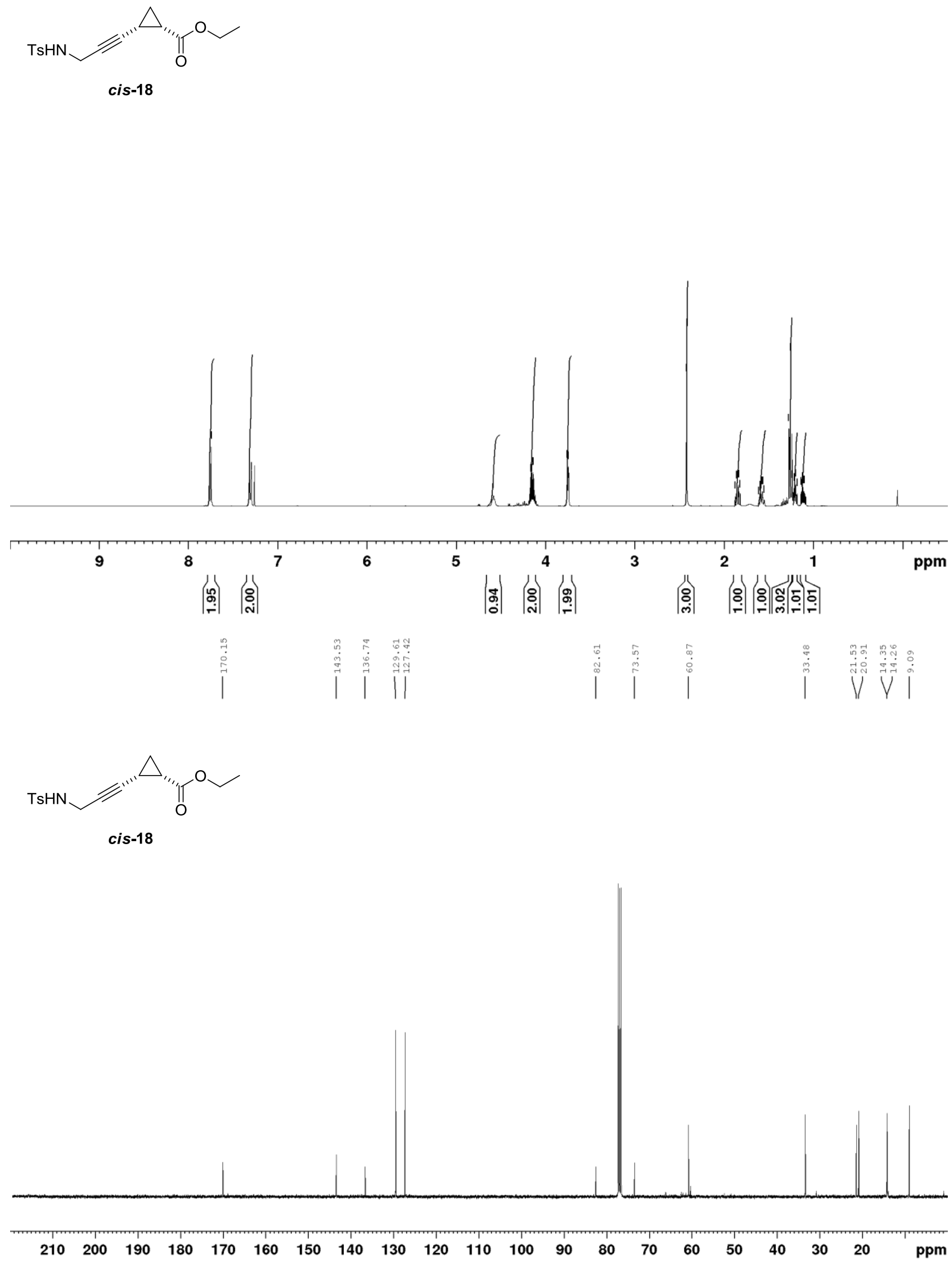

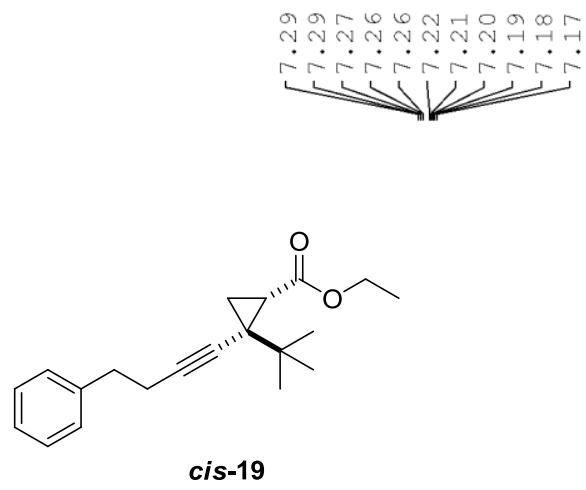

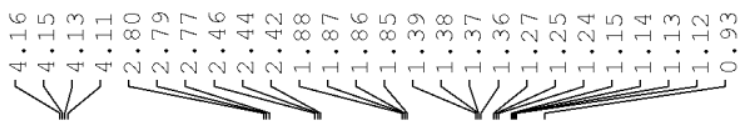

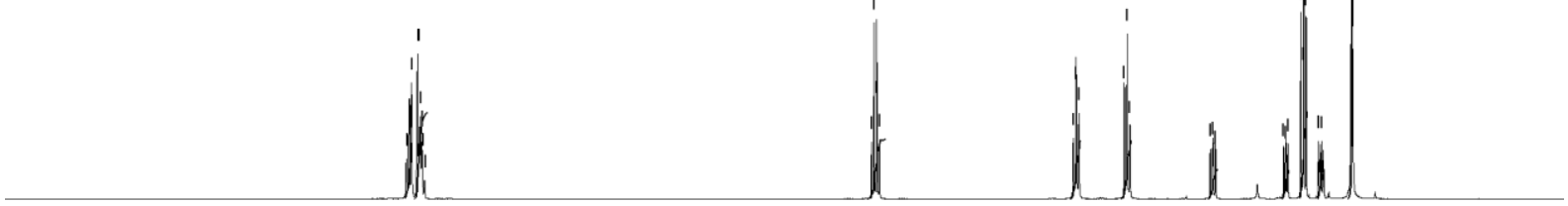
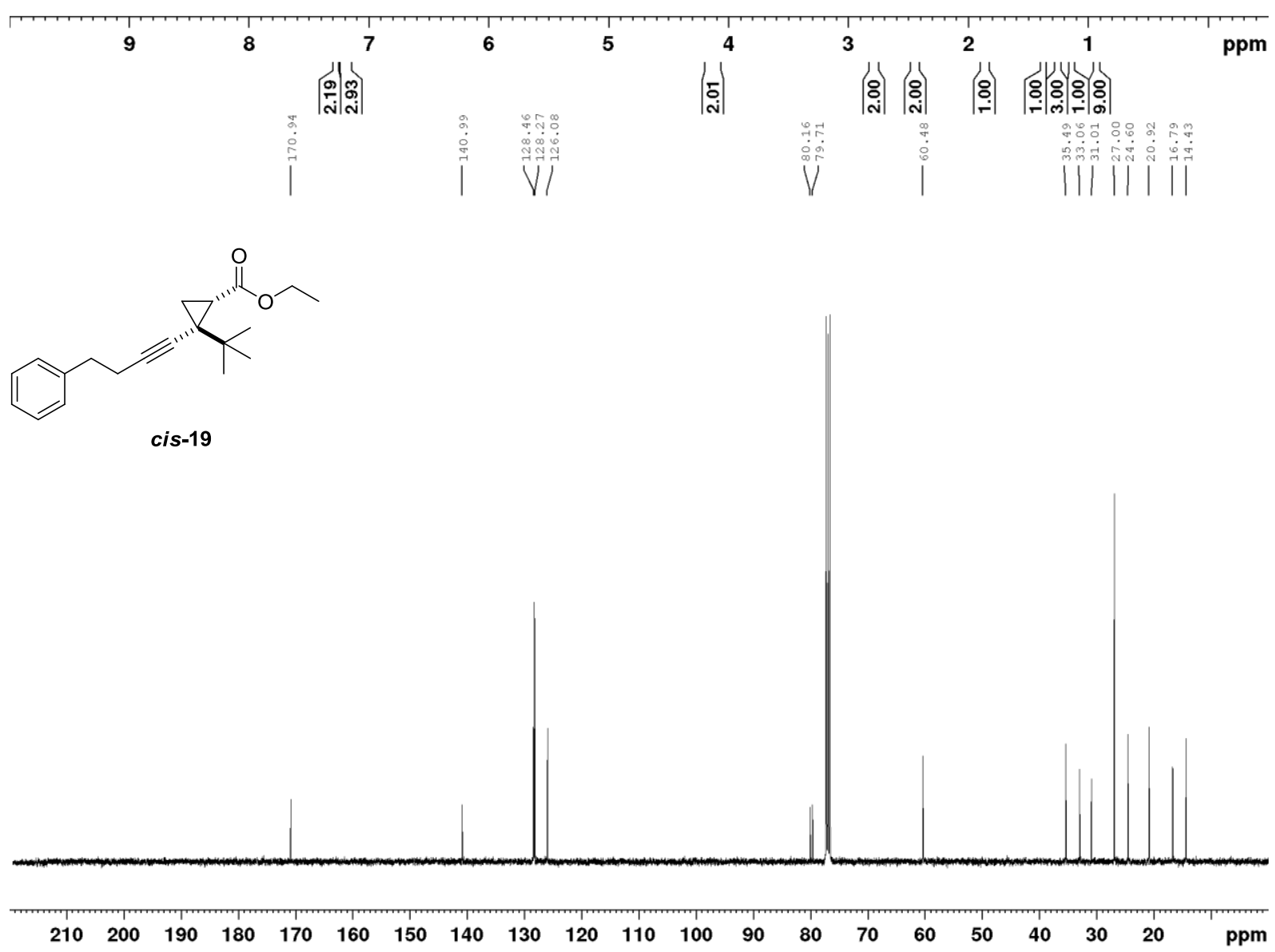

S105 


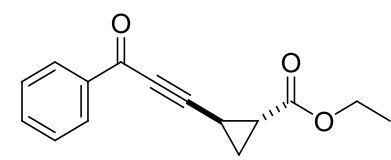

trans-20
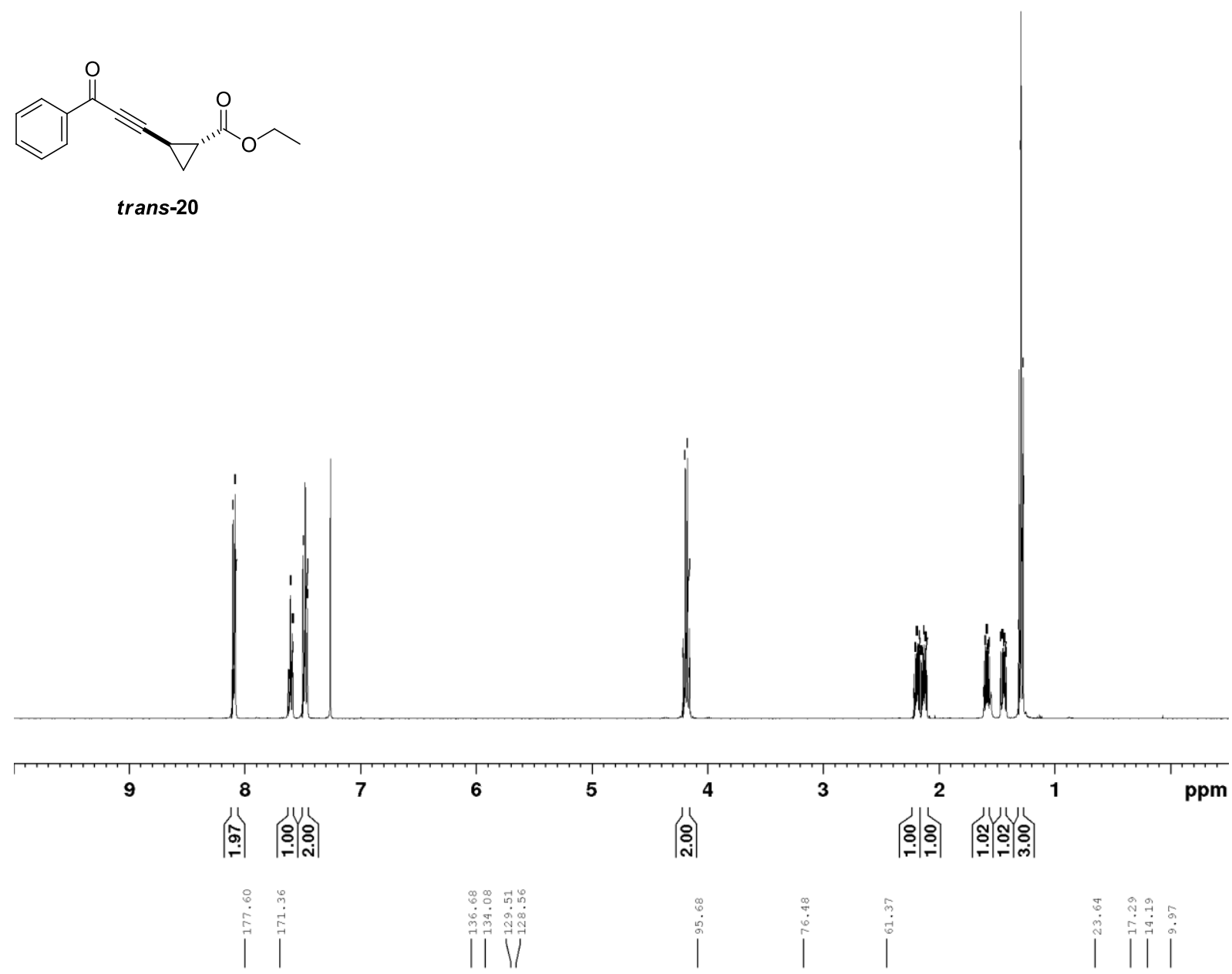

$>^{(1)}$

trans-20

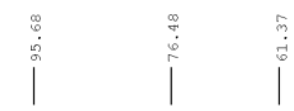

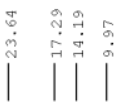

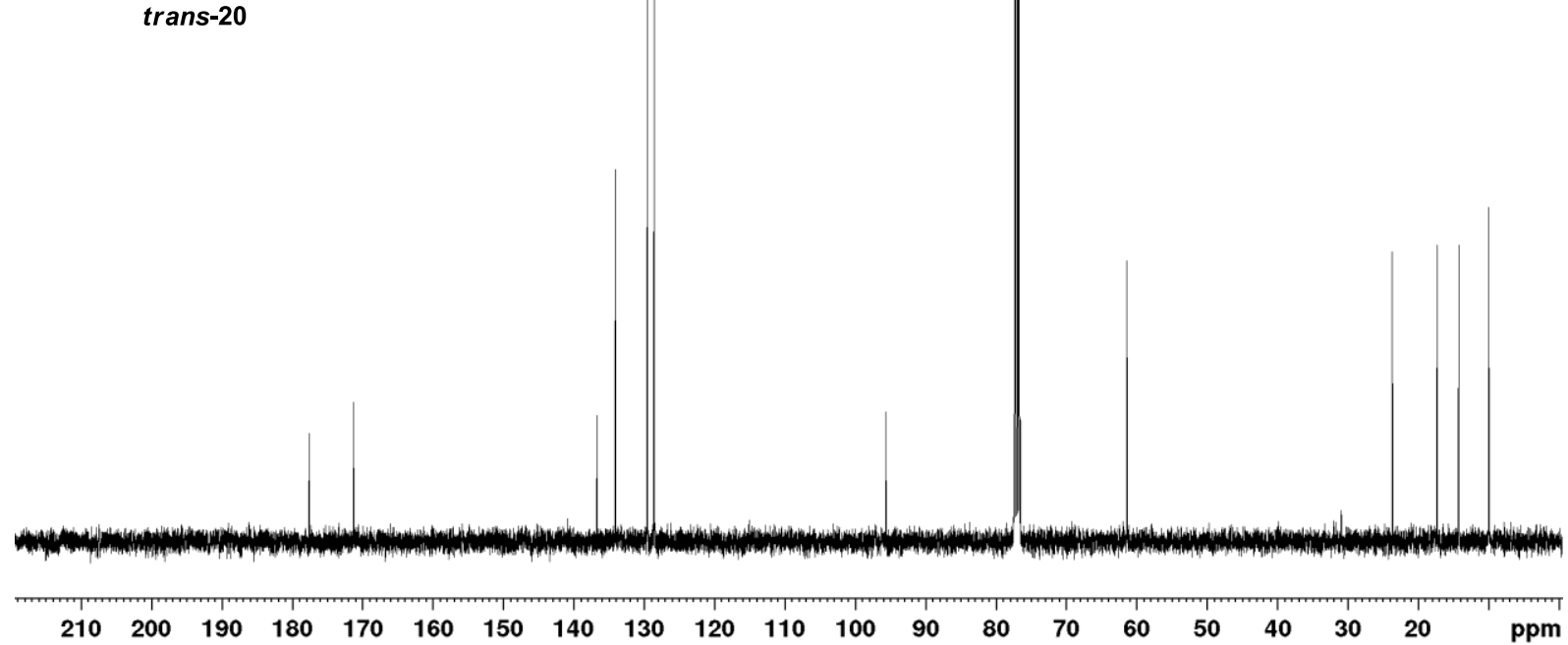


cis-20
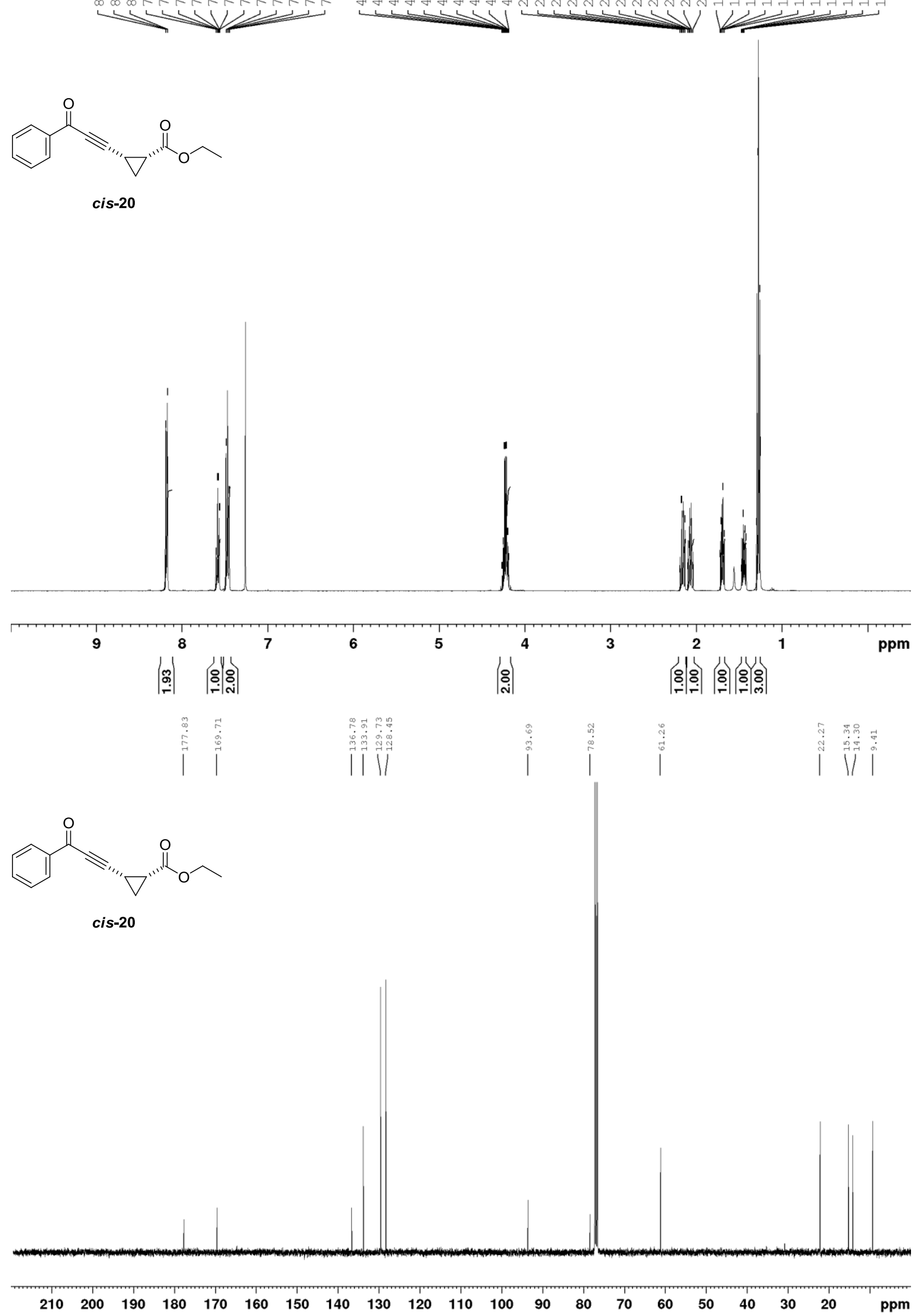


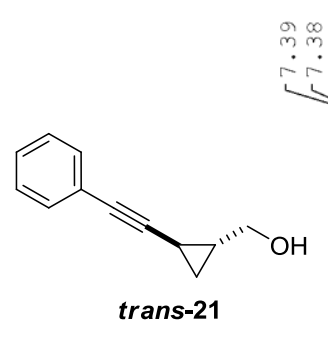

rans-21
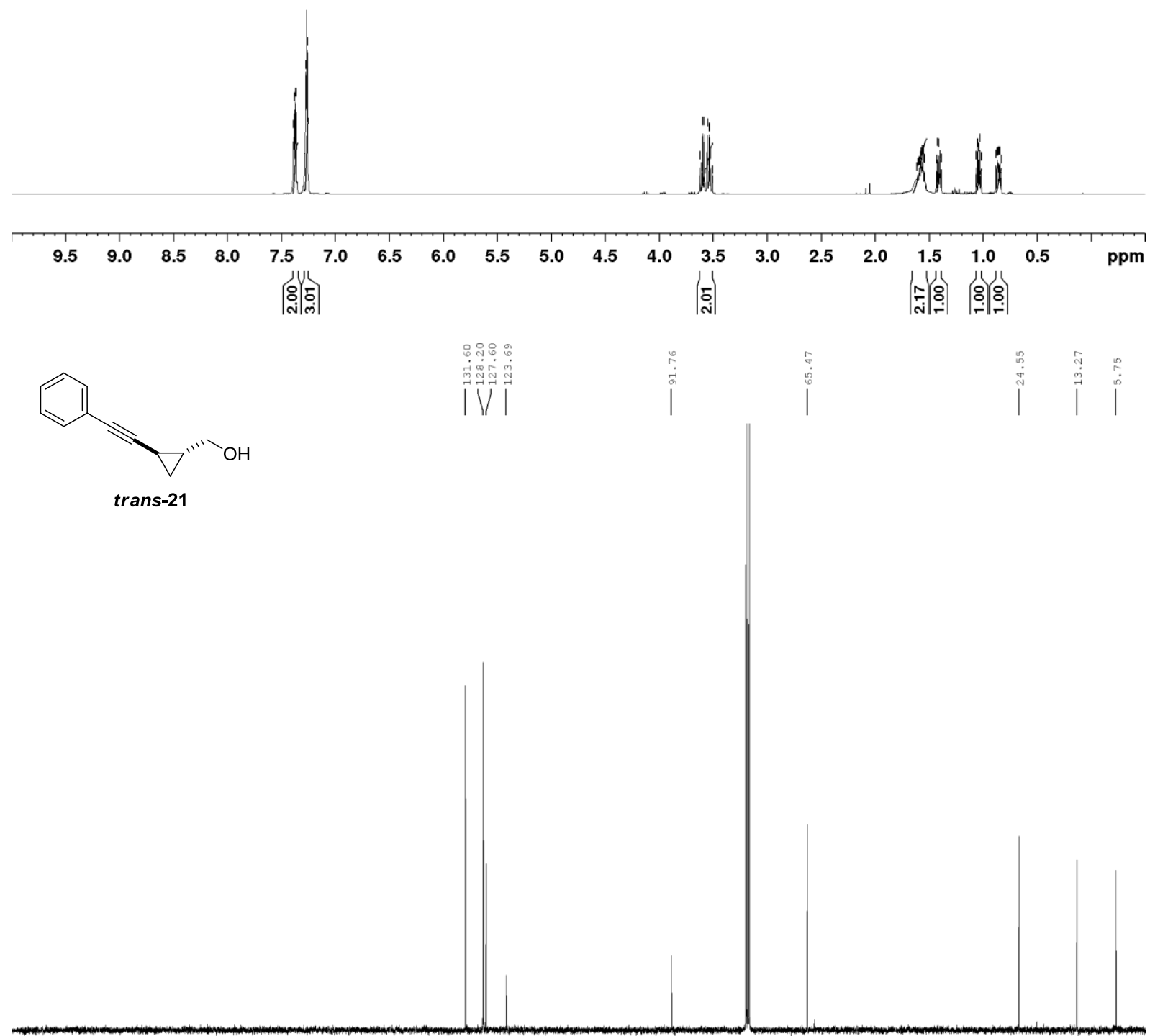

$\begin{array}{lllllllllllllllllllllll}210 & 200 & 190 & 180 & 170 & 160 & 150 & 140 & 130 & 120 & 110 & 100 & 90 & 80 & 70 & 60 & 50 & 40 & 30 & 20 & \mathrm{ppm}\end{array}$ 


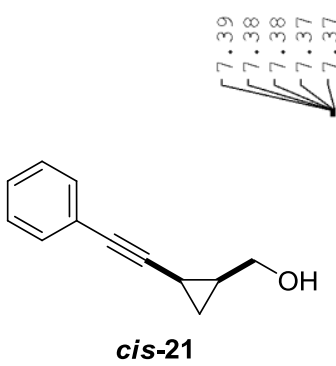

cis-21
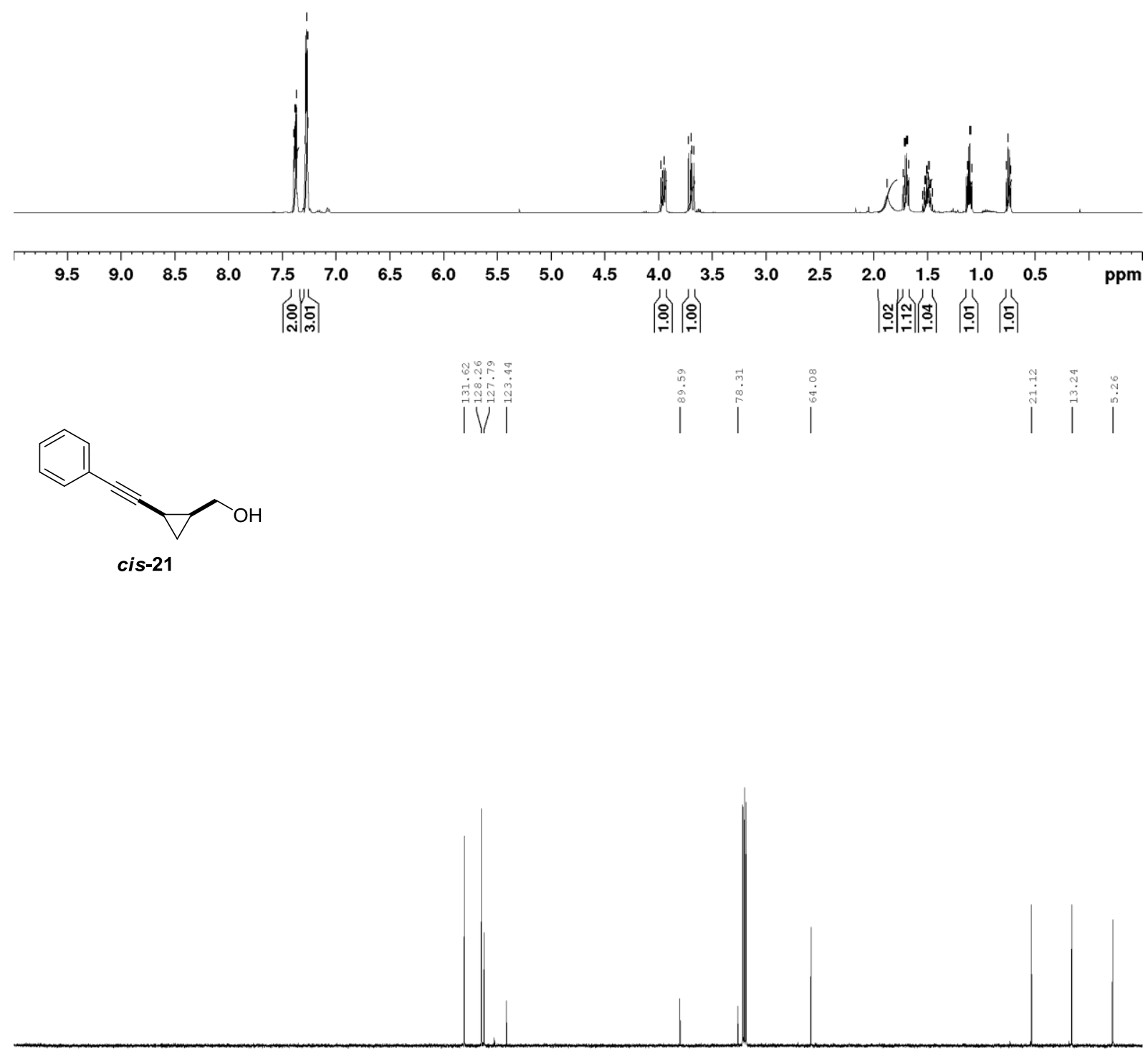

$\begin{array}{llllllllllllllllllllllll}210 & 200 & 190 & 180 & 170 & 160 & 150 & 140 & 130 & 120 & 110 & 100 & 90 & 80 & 70 & 60 & 50 & 40 & 30 & 20 & \mathrm{ppm}\end{array}$ 

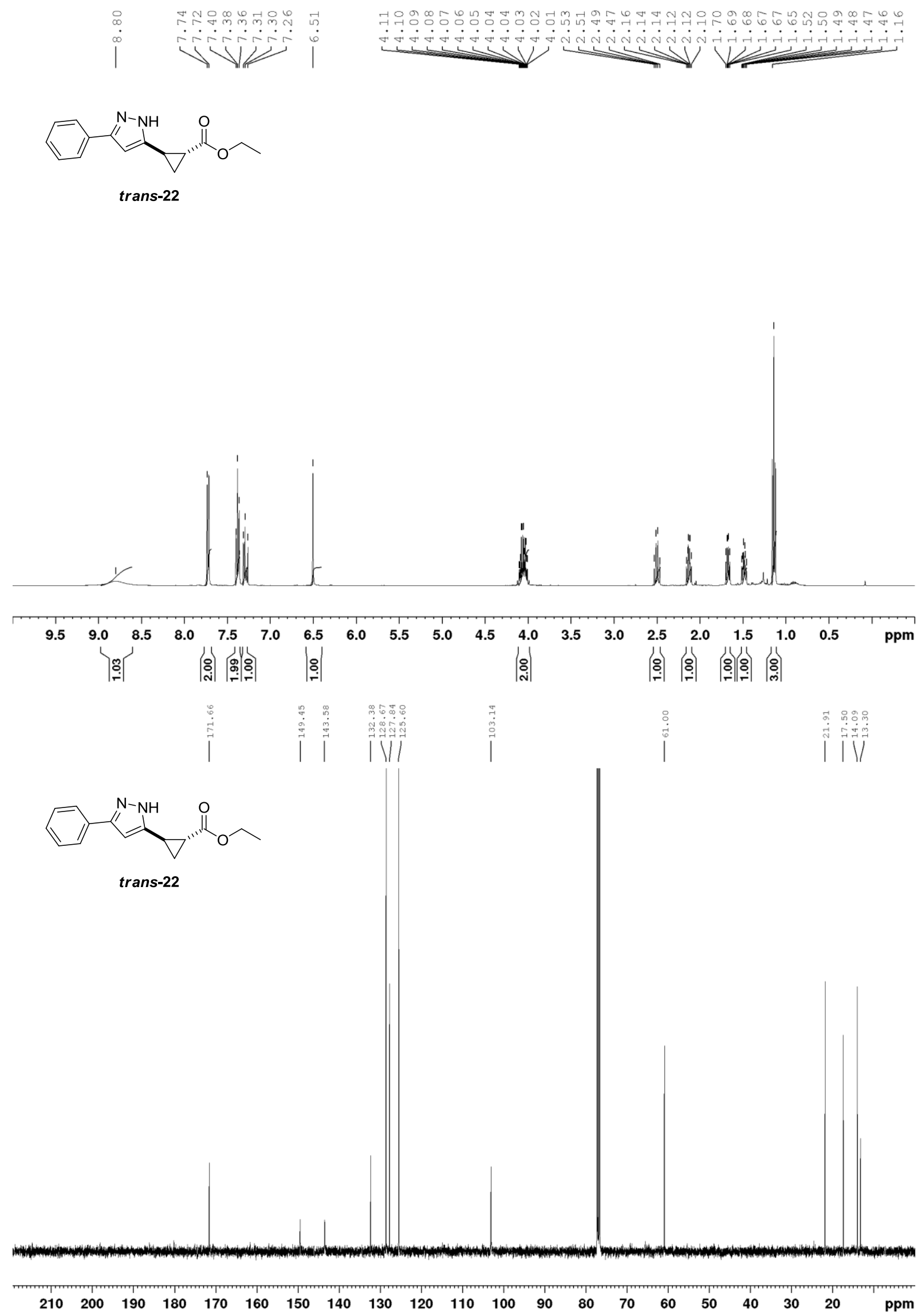

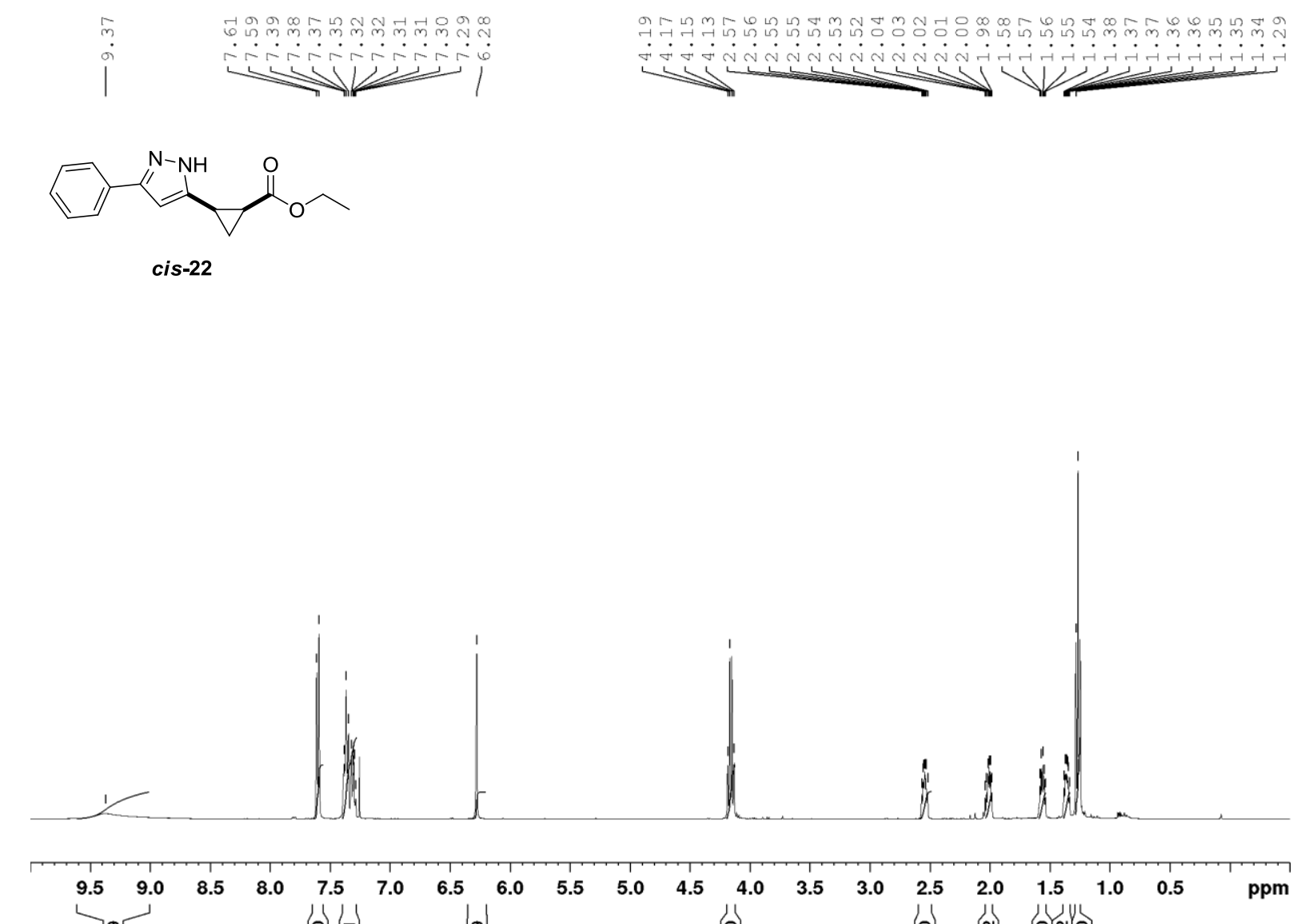
†:

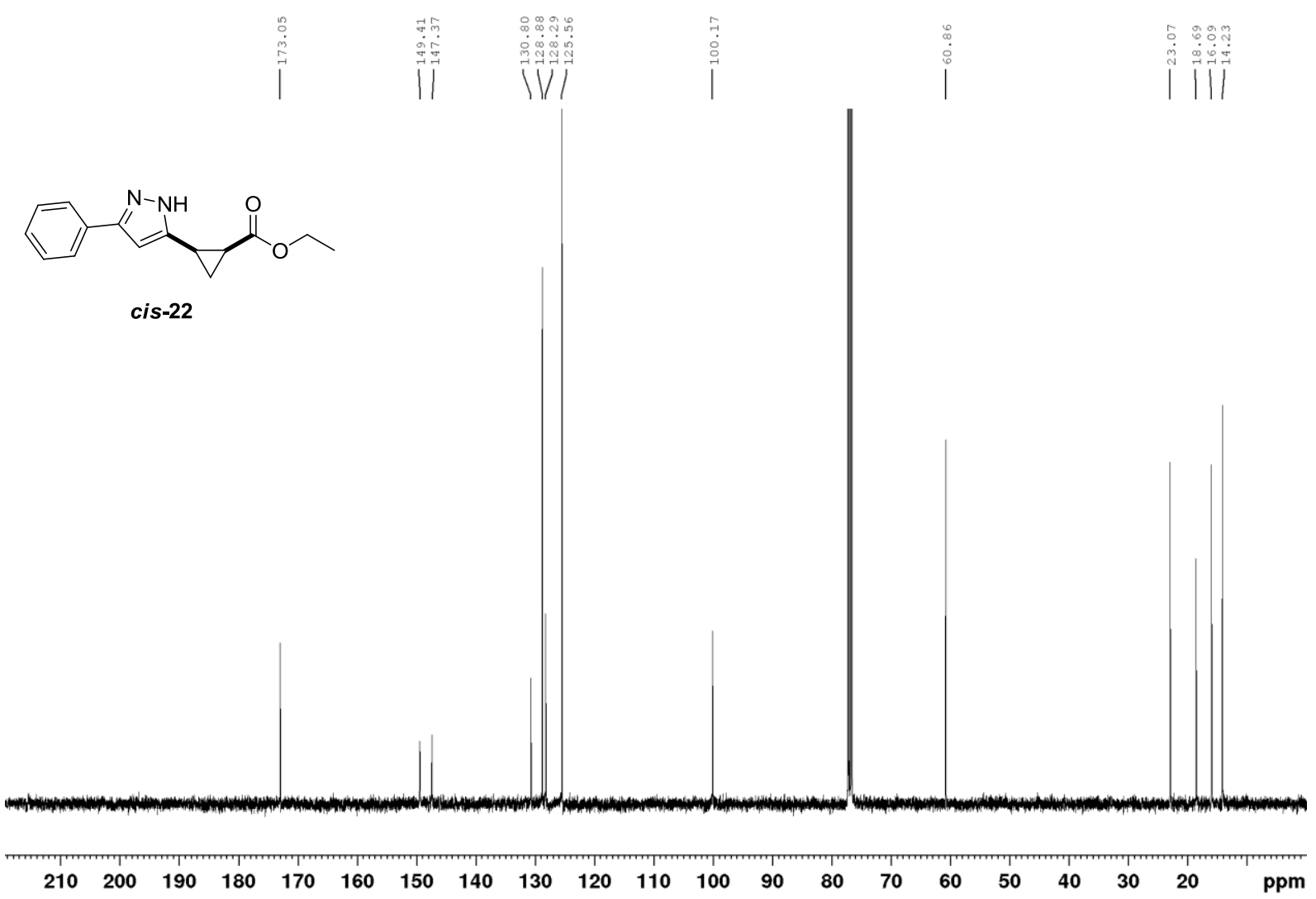

